Shape selectivity and lifetime in methanol to hydrocarbons conversion over medium pore zeolites

\title{
Dissertation for the degree of
}

\author{
Philosophia Doctor
}

Wegard Skistad

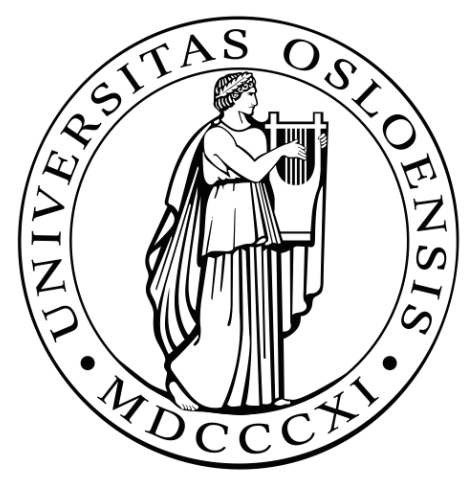

Departement of Chemsitry

UNIVERSITY OF OSLO 
(C) Wegard Skistad, 2014

Series of dissertations submitted to the

Faculty of Mathematics and Natural Sciences, University of Oslo No. 1528

ISSN 1501-7710

All rights reserved. No part of this publication may be

reproduced or transmitted, in any form or by any means, without permission.

Cover: Inger Sandved Anfinsen.

Printed in Norway: AIT Oslo AS.

Produced in co-operation with Akademika Publishing.

The thesis is produced by Akademika Publishing merely in connection with the thesis defence. Kindly direct all inquiries regarding the thesis to the copyright holder or the unit which grants the doctorate. 


\section{Preface}

The work presented in this thesis was carried out at the research center for Innovative Natural Gas processes and Products (InGAP), Departement of Chemistry, University of Oslo with 3.5 years funding (October 2009 - April 2013) from the Research Council of Norway and Industrial partners of InGAP. The PhD study comprised two years of research, one year of courses and 6 months industrial training at the R\&D facilities at Haldor Topøse. The work was submitted in May 2014 after one year work in the Norwegian school system.

The work presented here is a result of extensive collaboration between Industry (Haldor Topsøe) and Academia (University of Oslo and University of Turin). Karl Petter Lillerud, Stian Svelle, Merete Hellner Nilsen and Unni Olsbye have been my supervisors. All of you are greatly acknowledged for your enthusiasm, availability, guidance and support throughout this period. Pablo Beato at Haldor Topsøe is greatly acknowledged for sharing ideas, guidance and giving me a great time under the industrial trainee period. A part of my $\mathrm{PhD}$ was carried out at the University of Turin in close collaboration with Silvia Bordiga and Frencesca Bonino. You are both greatly acknowledged for all help with the in situ Raman experiments. Francesca Lønstad Bleken and Shewangizaw Teketel were main authors on paper I and III. I am grateful for the collaboration resulting in these papers.

I would like to thank all my present and former collegues in the catalysis group for a nice working environment. A special thank is adressed to Shewangizaw Teketel for his important contribution to the presented work, for many discussions and for a great time in Copenhagen. Einar Andre Eilertsen also deserves a special thank for all the discussions with respect to heteroatom incorporation into zeolites and the many non scientific discussions we had. Thanks to Bjørn Tore Lønstad Bleken and Marius Westgård Erichsen, which I had the pleasure to get to know already during my master degree, and to my room mate Naresh Babu Muddada.

Thanks to my parents, family and friends for all the support you have given me. Finally thanks to my wife Silje for the patience and support you have given.

The printed thesis only has a limited number of color pages. Some of the graphs may be difficult to analyze in black and white. The thesis is therefore available online in full color version. 



\section{Content}

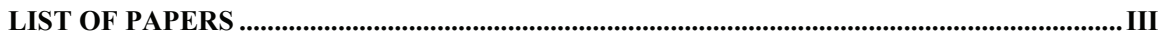

THE AUTHOR'S CONTRIBUTION ................................................................................................. IV

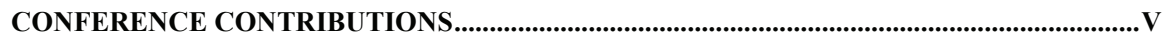

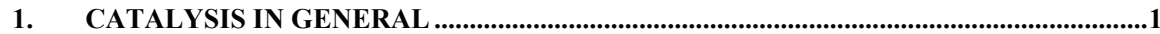

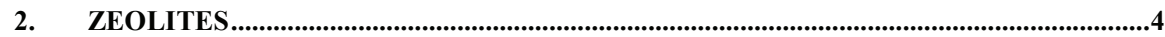

2.1 STRUCTURE AND PROPERTIES RELEVANT TO CATALYSIS ....................................................

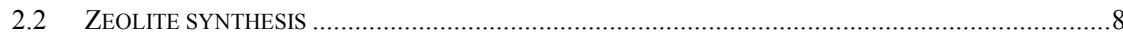

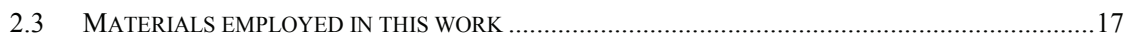

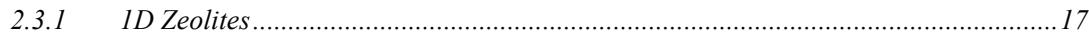

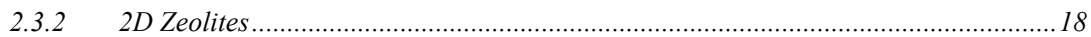

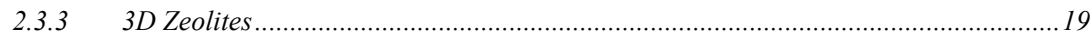

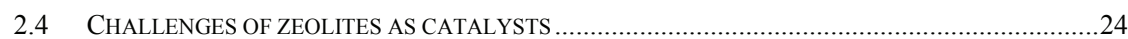

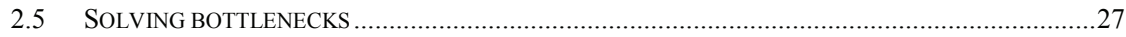

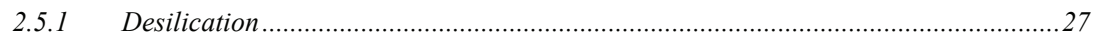

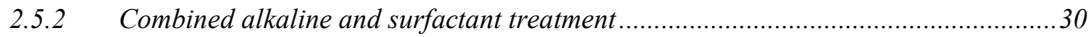

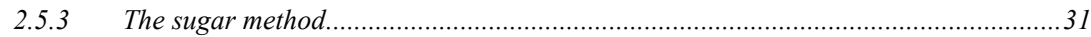

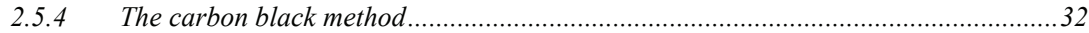

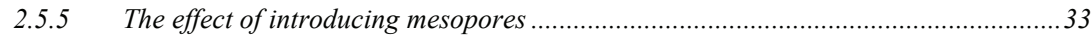

3. METHANOL TO HYDROCARBONS (MTH) ................................................................

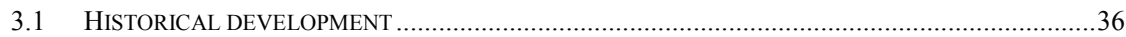

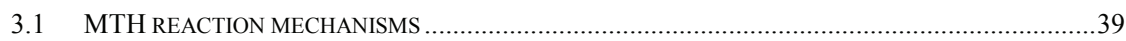

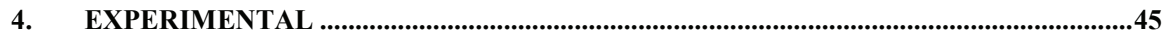

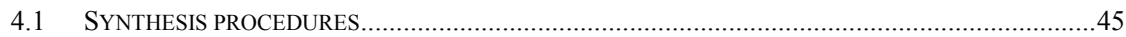




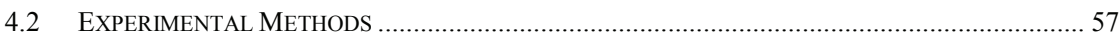

4.2.1 Characterisation methods ............................................................................... 57

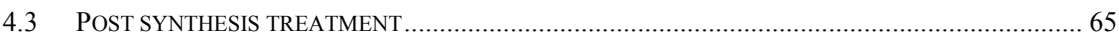

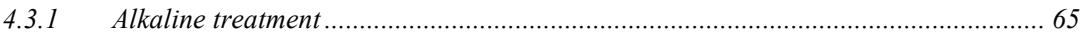

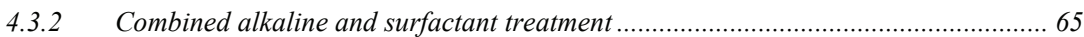

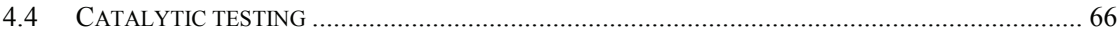

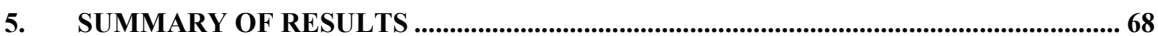

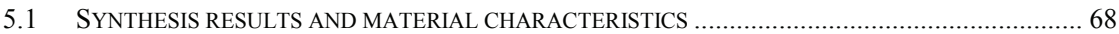

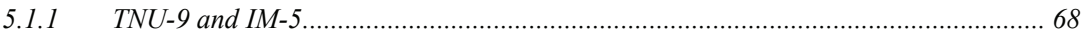

5.1.2 ZSM-23, ZSM-48, EU-1 (and ZSM-22) .......................................................... 75

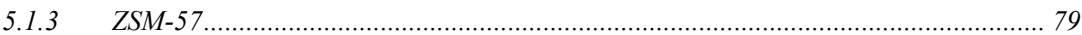

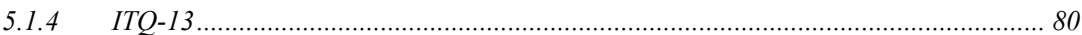

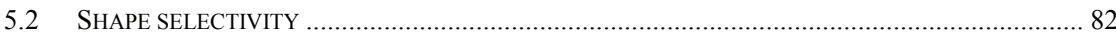

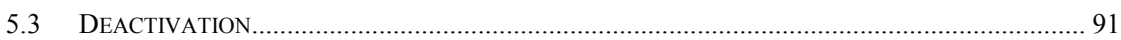

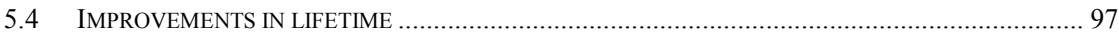

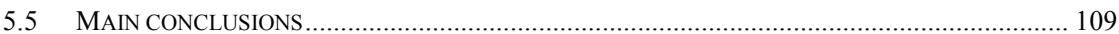

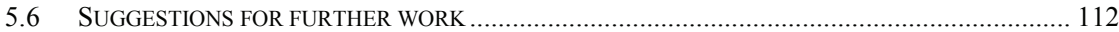

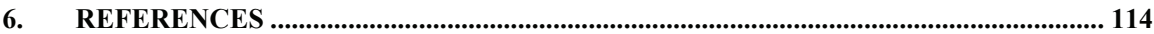




\section{List of papers}

Paper I: Shape Selectivity in the Conversion of Methanol to Hydrocarbons: The Catalytic Performance of One-Dimensional 10-Ring Zeolites: ZSM-22, ZSM-23, ZSM-48, and EU-1. S. Teketel, W. Skistad, S. Benard, U. Olsbye, K.P. Lillerud, P. Beato \& S. Svelle 2. ACS Catalysis (2012) s 26- 37

Paper II: Methanol Conversion to Hydrocarbons (MTH) Over H-ITQ-13 (ITH) Zeolite W. Skistad, S. Teketel, F. Lønstad Bleken, P. Beato, S. Bordiga, M. H. Nilsen, U. Olsbye, S. Svelle \& K. P. Lillerud. Topics in Catalysis (2013)

Paper III: Conversion of methanol over 10-ring zeolites with differing volumes at channel intersections: comparison of TNU-9, IM-5, ZSM-11 and ZSM-5. F. Bleken, W. Skistad, K. Barbera, M. Kustova, S. Bordiga , P. Beato, K. P. Lillerud, S. Svelle and U. Olsbye. PCCP 13 (2011) 2539-2549.

Paper IV: UV/Raman spectroscopic investigation of coke species formed over ZSM-5, TNU9 and IM-5 in the Methanol to Hydrocarbon reactions W. Skistad, F. Bonino, P.del Campo, P. Beato, U. Olsbye, S. Svelle, K. P. Lillerud and S. Bordiga.

Paper V: The influence of desilication and surfactant reassembly processes on the methanol to hydrocarbon reaction over ZSM-22, ZSM-23, EU-1,TNU-9 IM-5, ZSM-5 and ITQ-13 zeolites. W. Skistad, S. Teketel, P. Beato, U. Olsbye, K. P. Lillerud and S. Svelle. 


\section{The author's contribution}

Several people contributed to the Papers and manuscripts in this thesis. A description of Wegard Skistad`s contribution to each work is presented below.

Paper I: Wegard Skistad has synthesized EU-1 and ZSM-48 catalysts, contributed to the characterization of the catalysts, interpretation of the results, and preparation of the manuscript.

Paper II: Wegard Skistad has synthesized the catalysts and performed low pressure catalytic tests. He has contributed to the characterization of the catalysts, planning of the experiments, interpretation of the results, and preparation of the manuscript.

Paper III: Wegard Skistad has synthesized TNU-9 and IM-5 catalysts, contributed to the characterization of the catalysts, interpretation of the results, and preparation of the manuscript.

Paper IV: Wegard Skistad has synthesized TNU-9 and IM-5 and characterized the materials. He built a methanol pulse dosing system and performed the Raman spectroscopic investigation together with Francesca Bonino (University of Turin). He has contributed to the planning of the experiments, interpretation of the results, and preparation of the manuscript.

Paper V: Wegard Skistad has synthesized ZSM-23, EU-1, TNU-9, IM-5 and ITQ-13 catalysts and performed catalytic tests over the materials. He has contributed to the characterization of the catalysts, planning of the experiments, interpretation of the results, and preparation of the manuscript. 


\section{Conference Contributions}

Synthesis and Testing of Zeolites with Different Pore Systems - Methanol To Hydrocarbons.

W. Skistad, S. Teketel, S. Benard, Merete Hellner Nilsen, S. Svelle, K.P. Lillerud. Norwegian Catalysis Symposium, Bergen, Norway, 2010/11/29-30 (Oral presentation)

\section{Related papers}

Morphology Induced Shape Selectivity in Zeolite Catalysis. S. Teketel, L. F. Lundegaard, W.

Skistad, U. Olsbye, K. P. Lillerud, P. Beato, S. Svelle. Manuscript in preparation 


\section{Catalysis in general}

Chemistry is all about the fact that bonds break and bonds form. There are fundamental driving forces that can be applied to all chemistry independent of all the different kinds of classifications learned (condensation, addition, substitution, eliminaion reactions) as electrons like to move from electron dense areas to more positive sites. When these electrons change positions chemistry is going on. Many imagine that when electrons are removed from one bond to make another bond they carry energy in their bags and with this energy interesting things can happen (e.g. create new bonds). It is not telling the truth, because the real story involves the relative organization of charges to each other as well as other energy related features, however it is a concept that allows us understanding that chemistry is about moving electrons. So is also catalysis where the key concept is to control the electron flow to facilitate specific bond weakening.

Many of the reactions in industrial processes producing goods on which the society relies are naturally slow and in such reactions a high energy input is in principle necessary to improve the rate of reaction. For instance breaking the $\mathrm{C}-\mathrm{H}$ bond in methane in the steam reforming processes does not happen directly. Energy is needed to make the bond vibrate such that the electrons lose their connection to the protonic nuclei and move closer to another positive site somewhere else in the nearby surroundings. Sometimes cost related to the input of energy for accelerating reactions can be so high that it makes no sense to utilize commercially. However, history shows many successful developments and commercialization of products based on investigations on ways to change the flow of electrons, such that the energy required to initiate the reaction could be lowered. One of the most obvious ways to change the electron flow has been to introduce another specie or material that can steal or donate electrons (partially or totally) from the reactants. Materials or species that change the flow of electrons, and which result in accelerating reactions by lowering energy barriers (do not alter the overall stoichiometry and will be available for new cycles) as shown in Figure 1 are called catalysts 


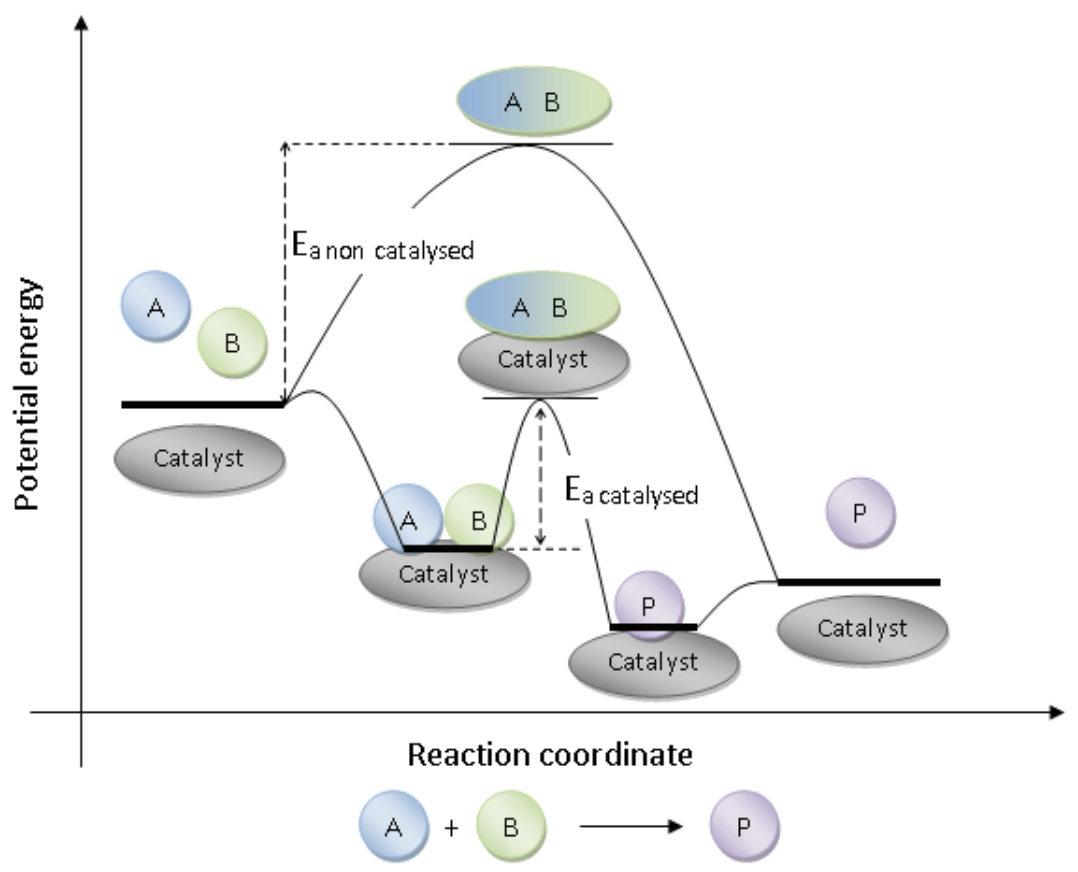

Figure 1: A general representation of a catalyzed versus non catalyzed reaction. Molecules collide with the catalyst, lose some of their energy to the surface and get adsorbed. In this way the bond breaking can be easier. This results in a lower activation barrier for the catalyzed reaction ( $\left.E_{a \text { catalyzed }}\right)$ than the activation barrier for the non catalyzed reaction ( $E_{a}$ none catalyzed $)$ which accelerates the reaction.

The catalytic route is more complex, involving additional steps towards the end products. In heterogeneous catalysis the molecule must collide with the surface and lose some of its energy (to the surface) to be sorbed. From here it migrates over the surface to find the spot (active site and eventually other reactants to react with) where the reaction takes place. The product may then desorb. Making bonds between the surface and the reactants or products can not be too weak (they will not adsorb), neither can the bonds be too strong (they will not desorb again). This is why balancing the electron flow in bond making is key in catalysis. The number of ways possible for tuning the strength of bonds in a fashionable way is the explanation why catalysts are the working horses in important industries worldwide e.g. the fertilizer industry, oil refineries or gas conversion industries.

In the last century turnover rates was of main focus in catalysis. This picture has changed and today selectivity towards desired products in catalysis is of increasing 
importance and is one route to efficiently reduce waste in chemical production.[1] From this perspective catalysis may not only lead to higher reaction rates, but facilitate reaction routes leading to both lower energy consumption and less byproducts. The everincreasing personal consumption calls for highly efficient production processes leading to less environmental impact. Material science and development of advanced catalytic processes is one important aspect in succeeding in these issues. 


\section{Zeolites}

\subsection{Structure and properties relevant to catalysis}

In mid 1950s the major use of catalysts was in the petroleum industry aiming to convert heavy petroleum fractions to transportation fuels, typically over an acidic silica-alumina gel. [2] The acidic function appeared when $\mathrm{Na}^{+}$ions associated with aluminate ions were replaced by $\mathrm{NH}_{4}{ }^{+}$with subsequent heat treatment to remove $\mathrm{NH}_{3}$ leaving an $\mathrm{O}-\mathrm{H}$ acidic group able to initiate reactions. Later on zeolites (Zeolite $\mathrm{X}$ ) were discovered to boost cracking reactions exceeding the results obtained over silica-alumina based catalysts in the laboratories of Union Carbide.[2] Further research led to Zeolite Y, a modified version of Zeolite $\mathrm{X}$ containing less aluminum and having mesopores present which boosted all previously results obtained.[2] Since then Zeolite Y (in an ultrastable version USY) has been the main component of the catalysts applied in the oil refineries around the globe.

Among many heterogeneous catalysts needed to accelerate industrial processes, zeolites have shown to be one of the most important catalysts. They are not only attractive for oil refineries, but also for upgrading biomass, coal and natural gas to valuable hydrocarbons, redox processes (and for ion exchange purposes). It is also the family of materials studied in this thesis converting methanol to hydrocarbons (MTH) as we will come back to later. To explain why zeolites are so attractive and the origin of their attractive properties, a closer look to the nature of this mineral family is needed. According to the Subcommittee on Zeolites of the International Mineralogical Association, Commission on New Minerals and Mineral Names a zeolite is defined as follows [3]:

A zeolite mineral is a crystalline substance with a structure characterized by a framework of linked tetrahedra, each consisting of four $\mathrm{O}$ atoms surrounding a cation. This framework contains open cavities in the form of channels and cages. These are usually occupied by $\mathrm{H}_{2} \mathrm{O}$ molecules and extra-framework cations that are commonly exchangeable. The channels are large enough to allow the passage of guest species. In the hydrated phases, dehydration occurs at temperatures mostly below about $400^{\circ} \mathrm{C}$ and is largely reversible. The framework may be interrupted by $(\mathrm{OH}, \mathrm{F})$ 
groups; these occupy a tetrahedron apex that is not shared with adjacent tetrahedra.

In principle zeolites are three dimensional crystalline and microporous aluminiumsilicates with ordered channels and cavities and can be described by the formula 1 :

$$
\left|M_{\frac{x}{n}}^{n+}\left(H_{2} O\right)_{z}\right|\left[A l_{x} S_{y} O_{2(x+y)}\right]
$$

The structure is typically described as corner sharing $\mathrm{TO}_{4}$ tetrahedra (see Figure 2) where the $\mathrm{T}$ atom can be a silicon or an aluminum atom (Eventually it is described by secondary building units or composite building units which are larger repeating fragments making up the structure.)

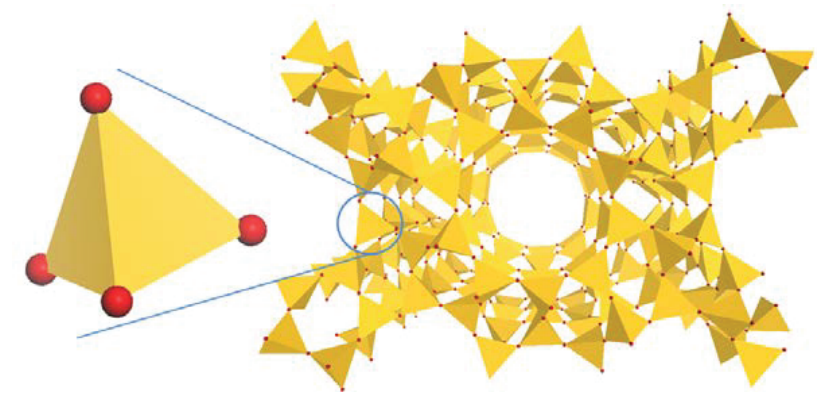

Figure 2: Corner sharing tetrahedrons are linked together to a zeolite framework. The central atom often denoted the Tatom, is a Si or an Al atom. Also heteroatoms (other elements which can replace Al or Si as a central atom) can be positioned in $T$ atom positions.

If a pool of tetrahedra were available and a task was given to put them together to structures the great variety and flexibility in the numbers of structures possible to obtain would have been easily discovered. Some structures could be rather dense with narrow channels or small cages while other structures could have had channels and cages of large sizes and some could be intermediate. Several million hypothetical zeolite structures were recently predicted by computer modeling; more than 450000 should be stable when compared to known zeolites.[4, 5] Thus it is a good chance to find new interesting zeolites with pore size distributions optimized for specific catalytic purposes.

The variety and flexibility is especially interesting because those channels and cavities found are of molecular dimensions, typically between 0.2-1 nm.[6] In other 
words zeolites can act as molecular sieves excluding larger molecules than the size of the channels and including molecules which are smaller than the channel openings. The size of zeolite channels are often described by the number of $\mathrm{T}$ atoms defining the pore aperture, 8-rings are counted as small pored, 10-rings as medium pored, 12-rings as large pored and even larger rings are classified as extra large pored.[7] By controlling the pore size distribution the structure could let in only those molecules we want to react, thereby controlling one parameter affecting the product distribution.

Inside the zeolite pore system, molecules will build up under continued feed of reactants, and obviously those molecules can only grow to sizes restricted by the size available in the channels and cavities. In this way the type of zeolite also controls the size of the transition states involved in the reaction. Finally when the products are formed the diffusion out through channels will depend on the relative size of the products to the pore size exposed to the exit of the crystals. In this way molecules formed in cavities which are larger than the channels may be trapped inside while smaller products can diffuse out. These three parameters influencing on the selectivity are called reactant selectivity, transition state selectivity and product selectivity as shown in Figure 3.
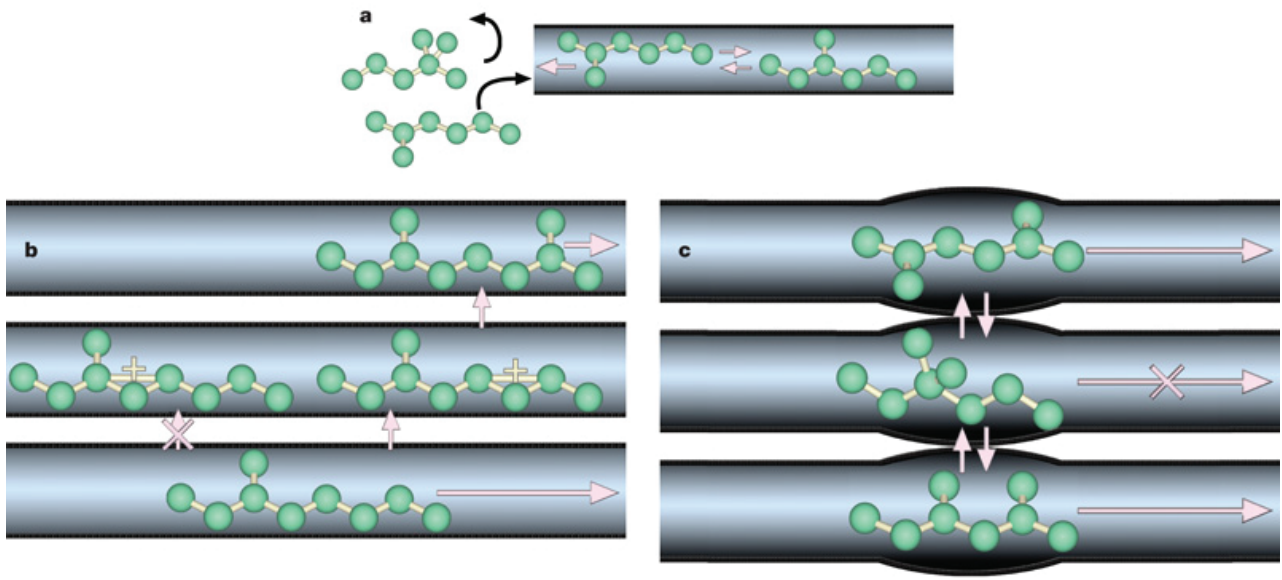

Figure 3: The selective properties of zeolites involve reactant selectivity (a), transition state selectivity (b) and product selectivity (c). [8] Reactant selectivity: the reactants are too large to enter the inner pore system. Transition selectivity: Intermediates too large to fit into the channels of the zeolite will not form while those with size smaller than the channel may form. Product selectivity: Molecules formed inside cavities may be larger than the aperture of the channels leading out of the zeolite and thus be stuck or only slowly diffuse. 
Although the open framework in zeolites gives rise to the attractive selective properties there is a need of additional properties to play a role as a catalyst. Silicate structures lacking aluminum can have similar pore systems, but they do not act as catalyst as the zeolites do because the surface is homopolar with no clear sites attracting or donating electrons to initiate and accelerate reactions (at least not very active). Zeolites on the other hand have sites which change the flow of electrons. This appears because the Al ${ }^{(3+)}$ has a lower valency than $\mathrm{Si}^{(4+)}$ and for every $\mathrm{Al}$ that is taking the position of a $\mathrm{Si}$ a net negative charge appears in the framework. No minerals does in principle exist when it is charged so the negative charge needs to be balanced by cations represented by $\mathrm{M}^{\mathrm{n}+}$ in the formula mentioned above. In the many natural existing zeolites these ions are typically alkali metal ions or earth alkali metal ions like $\mathrm{Na}^{+}, \mathrm{K}^{+}, \mathrm{Li}^{+}, \mathrm{Mg}^{2+}$ or $\mathrm{Ca}^{2+}$ since these ions are common in the crust. Multivalent cation exchanged zeolites were known to boost acid-catalytic activity already in 1957 when Union Carbide tested Ca-exchanged X zeolite as a catalyst for cracking of cumene (isopropylbenzene) into benzene and propene.[2] The reason for this activity was explained by the hydrolysis of the multivalent cations to $\left(\mathrm{Ca}^{2+} \mathrm{OH}^{-}\right)$which migrates to the sodalite cages, and the attack of the oxygen anions with a proton left from water to make an $\mathrm{O}-\mathrm{H}$ acidic site. It required a high loading of $\mathrm{Ca}^{2+}$ ions to first replace the $\mathrm{Na}^{+}$ions in stabilized positions in the double 6 rings (D6R) and then to migrate to the less favorable positions in the big $\alpha$-cage.[2] However often these ions do not lead to the catalytic properties which make zeolites so attractive because they result in low acidity. Therefore in the laboratory or in industry these ions are typically exchanged with protons (as shown in figure 1) which is the key ion for acid driven reactions as we find in e.g. fluid catalytic cracking unit in the oil refineries. In Zeolite $\mathrm{X}$ the very high $\mathrm{Al}$ content $(\mathrm{Si} / \mathrm{Al} \sim 1)$ made it impossible to ion exchange the $\mathrm{Na}^{+}$to $\mathrm{NH}_{4}{ }^{+}$ with subsequent thermolysis to obtain the proton form without structure collapse (because of the many defective sites the Al will give) and this was the reason why they used $\mathrm{Ca}^{2+}$.[2] When they finally succeeded to synthesize Zeolite $\mathrm{Y}$ rich in Si with the exact same topology, but containing less $\mathrm{Al}$, the structure could easily be ion exchanged to obtain the proton form without loss of crystallinity.[2] Direct ion exchange gave stronger acid sites than for the indirect hydrolysis taking place in Zeolite X. Thus the Zeolite Y was the catalyst applied. 

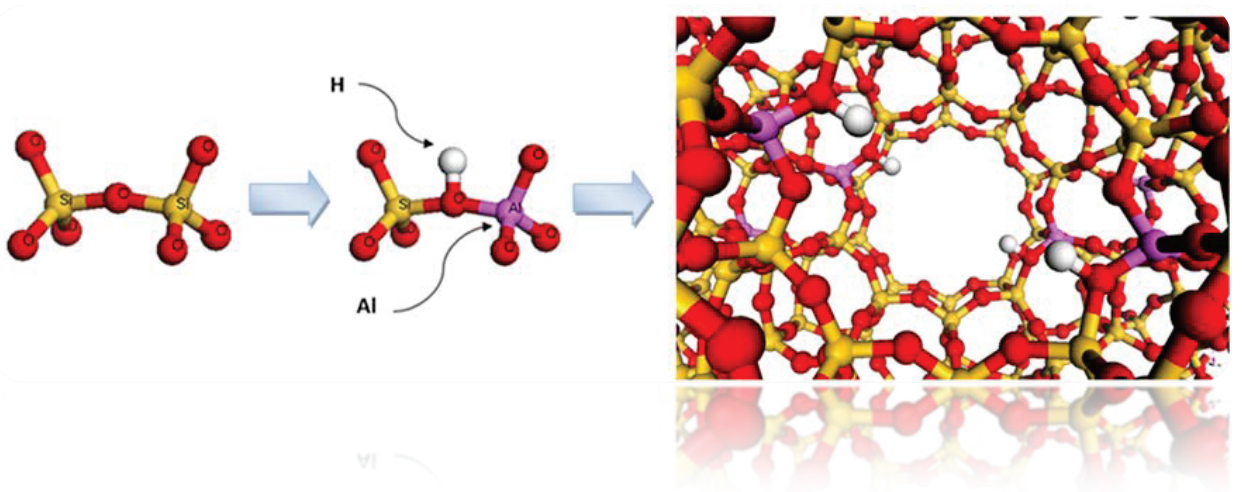

Figure 1: For every Al-atom substituting a Si-atom a negative charge appears in the framework. This negative charge has to be balanced by cations e.g. $\mathrm{Na}^{+}$, $\mathrm{Ca}^{2+}$ or as in this case proton.

The array of properties can be further extended. The alkali and earth alkali ions can be exchanged with other ions like $\mathrm{Cu}^{2+}, \mathrm{Fe}^{2+}$ and so on to get redox properties important for e.g. exhaust gas treatment like deNO $\mathrm{N}_{\mathrm{x}} \cdot[9-11]$

Crystals are rarely perfect and will contain defective sites. This means that also Lewis acid sites will be present in the crystal and might influence the reactions. According to the strict definition of zeolites only $\mathrm{Al}$ or Si can be present as $\mathrm{T}$ atoms, but we often count other element containing aluminiumsilicates as zeolites as well. Bifunctional catalysts like Ti- or V-zeolites combines both acid and redox properties and Ge containing zeolites stabilizing strained structural building units are examples of what is often also referred to as zeolites although they are not pure aluminiumsilicates. In the case of the lack of $\mathrm{Al}$ while other metals are present in tetrahedral positions the materials are denoted as vanadium silicates (in case of V), titanosilicates (in case of Ti), SAPO (in case of $\mathrm{P}$ ) etc. The zeolites and zeotypes are therefore materials rich in chemistry.

\subsection{Zeolite synthesis}

Many synthesis routes to form zeolites exist and they have much in common. A Si source (e.g. colloidal $\mathrm{SiO}_{2}$, sodium silicates, alkoxides, fumed $\mathrm{SiO}_{2}$, exoskeletons from diatoms) and an Al source (e.g. Bohemite, alkoxides, Al salts) is needed in all cases. Zeolites are often synthesized hydrothermally, which means that water is the solvent and 
that we operate at temperatures above the boiling point of water. In this way researchers try to mimic the ways of natural zeolite formation which often takes place in holes in volcanic rocks filled with pore water with species dissolved from the host rock and crystallize dependent on temperature, concentration and pressure.[12]

Because of the difficulties of many organic-inorganic interactions, great flexibility of the connection of tetrahedra to structures, the metastable nature of zeolites, solubility variations and the fact that we often lack in situ methods to follow crystallization mechanisms under such harsh and pressurized environments, the zeolite synthesis system is not fully understood. However two extreme mechanisms can be illustrated, Figure 4; (A) Solution-mediated transport crystallization and (B) solid-phase (gel) transformation crystallization mechanism.[13] In the solution-mediated mechanism dissolved species in a clear solution are assembling to nuclei and further to solid crystals which precipitate form the solution. This is not the case in the gel transformation mechanism where both nucleation and growth is believed to take place in the solid gel phase.[14]

(A)

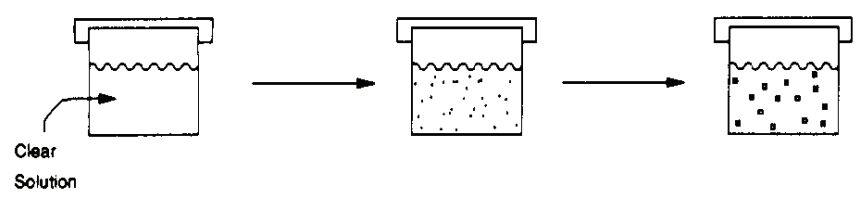

(B)

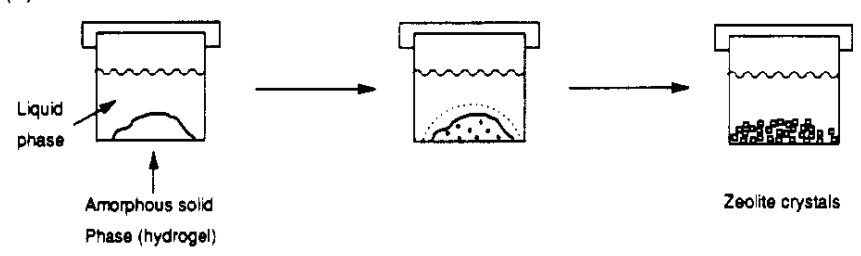

Figure 4: The two extreme mechanisms of zeolite formation: (A) Solutionmediated transport and $(B)$ solid-phase transformation crystallization mechanism. [13]

A schematic unification (valid for both the solution and gel mediated crystallization mechanisms) is shown in Figure 5. The reactants, a mineralizer and water (and eventually a SDA = structure directing agents, normally organic molecules which allow to select between phases of similar thermodynamic stability) is mixed into an 
autoclave which is closed and when the autoclave is heated (typically $100-200^{\circ} \mathrm{C}$ ) autogeneous pressure is created. Under such conditions amorphous reactants are slowly converted into zeolite crystals which typically are recovered by filtration followed by subsequent washing and drying.

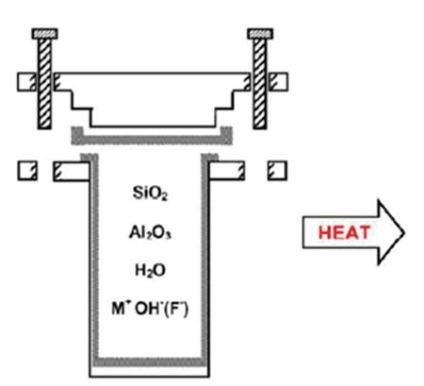

$\mathrm{Si}-\mathrm{O}, \mathrm{Al}-\mathrm{O}$ bonds

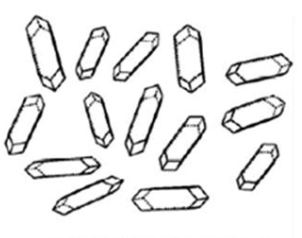

CRYSTALLINE ZEOLITE

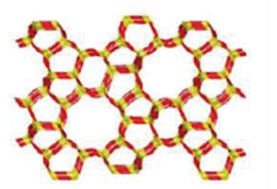

$\mathrm{Si}-\mathrm{O}-\mathrm{Al}$ bonds

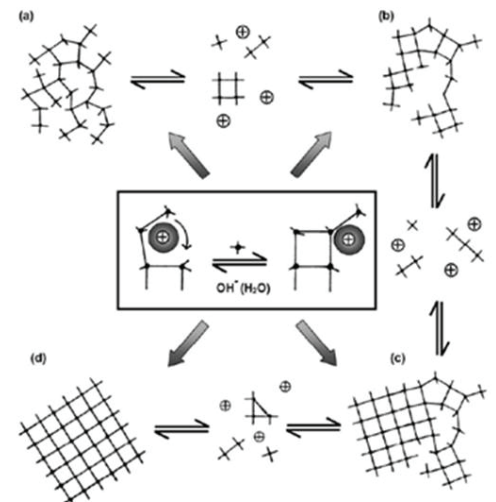

Figure 5: A representation of the general synthesis systems. Amorphous reactants are slowly converted into small nuclei with short range order and further growth to obtain a zeolite crystal with long range order. Structure directing agents are facilitating the growth.[14]

\section{Mineralizers}

In the above section the role of the mineralizer was not mentioned. This plays a crucial part in zeolite synthesis. A Sandy beach, mostly consisting of $\mathrm{SiO} 2$, does not easily dissolve in water. Therefore in zeolite synthesis a mineralizer is typically used to facilitate the dissolution of the Si source (or in general accelerate crystallization). Traditionally it is $\mathrm{OH}^{-}$from sources like e.g. $\mathrm{NaOH}$ and $\mathrm{KOH}$ that have been used, but also $\mathrm{F}^{-}$from e.g. $\mathrm{HF}$ and $\mathrm{NH}_{4} \mathrm{~F}$ now play an important role as a mineralizer.[15] Examples of the two groups of mineralizers is given in formulas 2-3.

$$
\begin{aligned}
& \left.\mathrm{SiO}_{2}(\mathrm{~s})+2 \mathrm{OH}^{-} \rightleftarrows \mathrm{Si}(\mathrm{OH})_{2} \mathrm{O}_{2}^{2-} \text { (or in general Si }(\mathrm{OH})_{4-n} \mathrm{O}_{n}^{n-}\right) \\
& \mathrm{SiO}_{2}(s)+6 \mathrm{HF}(a q) \rightarrow \mathrm{SiF}_{6}^{2-}(a q)+2 \mathrm{H}^{+}(a q)+2 \mathrm{H}_{2} \mathrm{O}(\mathrm{l})
\end{aligned}
$$

Mineralizers such as $\mathrm{OH}^{-}$are good complexing agents and can bring amphoteric oxides and hydroxides into solution without making them too stable and unreactive.[16] Thus, in 
addition to formulas (2-3) $\mathrm{Al}_{2} \mathrm{O}_{3}$ and $\mathrm{Al}(\mathrm{OH})_{3}$ are examples of species which can be brought into solution by the assistance of a mineralizer.

$$
\begin{aligned}
& \mathrm{Al}(\mathrm{OH})_{3}(\mathrm{~s})+\mathrm{OH}^{-}(a q) \rightleftarrows \mathrm{Al}(\mathrm{OH})_{4}^{-}(a q) \\
& \mathrm{Al}_{2} \mathrm{O}_{3}(\mathrm{~s})+3 \mathrm{H}_{2} \mathrm{O}(\mathrm{l})+2 \mathrm{OH}^{-}(a q) \rightleftarrows 2 \mathrm{Al}(\mathrm{OH})_{4}^{-}(a q)
\end{aligned}
$$

\section{Organic Structure Directing Agents - on the way to increase the $\mathrm{Si} / \mathrm{Al}$ ratio range}

In the early synthesis of zeolites inorganic cations were known to have a certain structure directing effect. While $\mathrm{Na}^{+}$typically favored the formation of sodalite, $\mathrm{K}^{+}$favored Chabasite and Zeolite L, and analcime could be obtained from a range of cationic environments.[16] Cations can coordinate with water and together they may act as pore fillers. In most cases where inorganic cations are present the structure may be open, but the high charge density of the water coordinated cations induces low silica zeolites as is the situation for the natural minerals typically having $\mathrm{Si} / \mathrm{Al}$ ratios below 5.[15] On the other hand natural silicates and zeolites with very low Al content are typically dense phases.

Later Barrer and Denny introduced organic molecules, which are typically alkylammonium cations, Figure 6, or ammonium ions, as structure directing agents.[17] Such ions could occupy a larger space (being less polar) and therefore give less stabilization of anionic charge resulting in a higher $\mathrm{Si} / \mathrm{Al}$ ratio than typically observed in syntheses without such organic bases.[16] Today a wide array of organic structure directing agents are available and the use of such organic molecules is now the common way of selective (at least to some degree) zeolite synthesis. In this perspective two factors can be said to have main importance; the $\mathrm{C} / \mathrm{N}^{+}$and, size and rigidity.

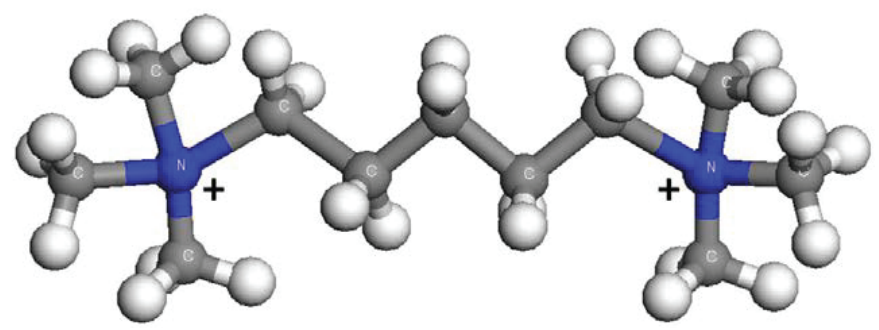

Figure 6: Example of a structure directing agent in zeolite synthesis. 
Work by Kubota et al.[18] has shown that, in the presence of $\mathrm{OH}^{-}$as a mineralizer, the preferred $\mathrm{C} / \mathrm{N}^{+}$ratio in the SDAs was between 11-15 for high silica phases. In their investigations they observed that the partitioning of SDAs (quaternary ammonium compounds) with different $\mathrm{C} / \mathrm{N}^{+}$ratios in chloroform and water turned out to be very different. [15] When the $\mathrm{C} / \mathrm{N}^{+}<11$ the transfer to the organic phase was very low, however when $\mathrm{C} / \mathrm{N}^{+}>15$ a high transfer to the organic phase was observed. Thus, the interaction with water and small SDAs with charge is strong compared to larger SDAs with similar charges. In other words, too weak interactions may lead to phase segregation while a high charge density typically leads to hydrophilic interaction allowing the SDA to make too strong water complexes. The strong interaction with water can be a plausible explanation why pure silica hydrophobic sieves are difficult to obtain with small organic ions such as tetramethylammonium ions $\left(\mathrm{TMA}^{+}\right.$) with the $\mathrm{OH}^{-}$route.[15] Thus optimum polarity lies in the intermediate range.

Clearly the use of organic bases has opened new possibilities to synthesise high silica zeolites, but to synthesise very high silica or all silica molecular sieves in $\mathrm{OH}^{-}$ media is a challenge. When the zeolite contains less Al, less negative charge is introduced to the framework.[15] Thus the positive charge on the SDA can not be stabilized by the negative framework charges anymore unless defective sites are present, e.g. Si-O- This instability often gives restrictions to the $\mathrm{Si} / \mathrm{Al}$ range obtained. The introduction of the fluoride route made it possible to synthesise zeolites and pure siliceous frameworks with wide ranges of $\mathrm{Si} / \mathrm{Al}$ ratios.[15] We remember that in $\mathrm{OH}^{-}$media the $\mathrm{C} / \mathrm{N}^{+}$ratio of 11-15 is preferred for obtaining high silica zeolites. However in fluoride media the situation is rather different. Even $\mathrm{TMA}^{+}\left(\mathrm{C} / \mathrm{N}^{+}=4\right)$ can act as SDA for the synthesis of very high silica zeolites.[15] The reason why the fluoride route works well to expand the Si/Al ratio further is because the $\mathrm{F}^{-}$interacts with strained structure units (e.g. D4R as is the case for ITQ-13[19]) and balances the positive charge on the SDA. Thus there is less or no need for defects to obtain total charge balance. 


\section{Flexibility and rigidity of the SDA}

Basically we can imagine that when moving from small and flexible to larger and rigid SDAs the structure directing nature is improving. High flexibility SDAs typically occur when the $\mathrm{C}$ atoms are at most secondary, in other words when it is a chain. Such SDAs typically direct several structures dependent on variations in the inorganic gel composition. Lee et al.[20] thoroughly studied the effect on gel composition on syntheses with diquaternary alkylammonium ions (a very common SDA group) $\left(\mathrm{CH}_{3}\right)_{3} \mathrm{~N}(+)$ $(\mathrm{CH} 2)_{\mathrm{n}}-\mathrm{N}(+)\left(\mathrm{CH}_{3}\right)_{3}$ with $\mathrm{n}=3-10$ as structure-directing agents. Only by varying the $\mathrm{NaOH} / \mathrm{SiO}_{2}$ ratio from 0.2-1.13 ZSM-48, ZSM-12, MCM-22, Mordenite and Analcime could be obtained with the same SDA containing $6 \mathrm{C}$ atoms in the chain. Even further variations in the number of structures were possible for each $\mathrm{NaOH} / \mathrm{SiO}_{2}$ ratio when varying the amount of Al. However, the SDA is not the only responsible for structure variations, since a given topology has a preferred most stable Al-content. The great flexibility of such SDAs gives many possibilities to find new structures not known by new flexible SDAs not studied in detail. However the rapid change of phase is often a frustrating moment for those of us that worked to expand e.g. Si/Al ratio keeping the same structure. Two of the most complex zeolites known to date, TNU-9 and IM-5, are synthesized by flexible SDAs.[21, 22] These two structures have been studied in detailed as a fundament for PAPER III. Despite large effort (change of template, composition, Ge incorporation, $\mathrm{HF}$ etc.) to expand the $\mathrm{Si} / \mathrm{Al}$ ratio from the very narrow range reported was very challenging without changing the phase.

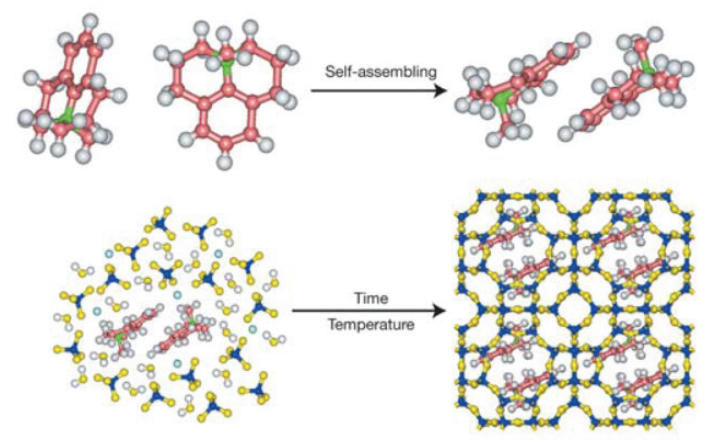

Figure 7: The formation of zeolite LTA based on supramolecular self-assembly of SDAs.[23] 
However in situations where tertiary $\mathrm{C}$ atoms are present, the SDAs get more rigid and have less conformational variability resulting in stronger structure directing nature. This is often utilized to make large and extra-large pore zeolites.[7] Unfortunately to synthesise the perfect SDA with right polarity and size is not straightforward. It often requires several synthesis steps to make such molecules and this may be expensive with respect to commercialization of the resulting zeolite. In some reports however, large and extra large pore zeolites may be formed by self assembly of smaller SDAs as shown for LTA in Figure 7.[7, 23]

\section{Introduction of heteroatoms}

Typically, in the case of $\mathrm{OH}^{-}$as a mineralizer the $\mathrm{pH}$ will be high, this is an important obstacle because of the hydrolysis of metals resulting in metal hydroxides or oxides which are difficult to incorporate in framework positions. In the fluoride route, the $\mathrm{pH}$ could tune in a wide range from acidic to basic when combined with weak organic bases to more strong organic bases often ion exchanged with $\mathrm{OH}^{-}$. In mild acidic environments metal ions will form complexes with $\mathrm{F}^{-}$. Both $\mathrm{Fe}$ and $\mathrm{Ga}$ react to give $\mathrm{MF}_{6}^{3-}$ while in case of e.g Ti $\mathrm{MF}_{6}^{2-}$ will be formed, which in further reactions with water results in oxyfluorinated compounds (e.g. $\mathrm{FeOF}_{4}^{3-}$ or $\mathrm{TiOF}_{4}^{2-}$ ). It is believed that such complexes combine with $\mathrm{SiOF}_{4}^{2-}$ leading to zeolite crystals with heteroatoms incorporated (not conclusively proven). The lower $\mathrm{pH}$ possible in the fluoride route has therefore given great possibilities for introducing transition metals in the framework such as $\mathrm{Sc}, \mathrm{Ti}, \mathrm{Fe}$ or metals such as B etc.[24, 25]

Recently $\mathrm{Ce}$ and $\mathrm{La}$, rather large heteroatoms, were introduced in framework positions $\left(\mathrm{OH}^{-}\right.$route) of MCM-22 (MWW) by a several stage synthesis shown in Figure 8.[26] By hydrolyzing TEOS in acidic environment ( $\mathrm{pH} 2$ ) with Ce salt or La salts gave metal ions isolated and dispersed in the solution. When adding the SDA the environment will be mildly acidic ( $\mathrm{pH} \sim 5-6)$ and Si-O-M-O-Si bonds form around the SDA which is the precondition obtaining for tetracoordinated metal ions. In a final step the $\mathrm{pH}$ is increased by addition of an inorganic base before crystallization ( $\mathrm{pH} \sim 11$ ) of $\mathrm{La}(\mathrm{Ce})$ MCM-22. 


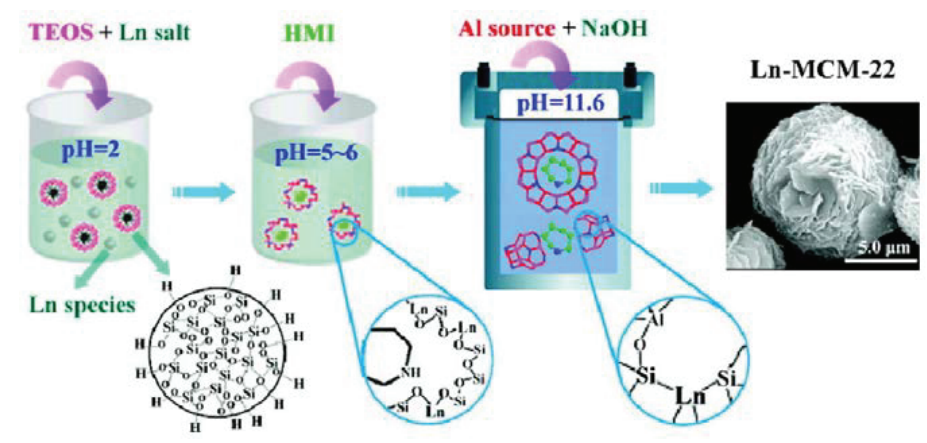

Figure 8: Introduction of (large) heteroatoms, La and Ce, in MCM-22 by a several step synthesis method.[26]

\section{The role of Ge in zeolite synthesis}

Until 1996 both the $\mathrm{OH}^{-}$and the $\mathrm{F}^{-}$route typically gave zeolites with high Framework Densities (FD $=$ number of tetrahedra per $\mathrm{nm}^{2}$ ), typically FD $\geq 17.3$.[15] Since then the combination of highly concentrated synthesis mixtures combined with the fluoride route has given many new structures with low FD $(\leq 17)$. [15] When calculating hypothetical frameworks and observing already known zeolites the FD can be correlated to the size of the smallest ring present in the framework.[7, 27, 28] Typically small rings evolve strain, but are essential for low FD zeolite type frameworks. Introduction of Ge has shown to stabilize smaller structural units such as D4R because of the smaller angle of the Ge-OGe $\left(130^{\circ}\right)$ or Ge-O-Si compared to Si-O-Si which in pure silica phases is $148^{\circ}$. Up to $3 \mathrm{Ge}$ atoms can stabilize the D4R and reduce the crystallization time, especially in combination with $\mathrm{F}^{-}$entering the strained unit.[19, 29-31] In germanates all four T- atoms can be Ge.

In 2010 the first zeolites containing double three rings (D3R) was synthesized by Jiang et al. [32] and Corma et al. [33] Such structural units will be extremely difficult to obtain with only $\mathrm{Si}$ or $\mathrm{Al}$ in the framework because of the large preferred $\mathrm{Si}-\mathrm{O}-\mathrm{Si}(\mathrm{Al})$ bond angle. Again the Ge allows stabilization of structural units with very small angles to form. This time it resulted in zeolites with very low framework densities (FD $10.1 \mathrm{~T} / 1000 \AA$ ) consisting of both $16 \times 15 \times 15$-rings in case of ITQ-40 and 10.9T/1000 with $18 \times 12 \times 12$ rings for ITQ-44.[32, 33] The good thing is that several interesting structures are likely to appear in the future because of the many hypothetical frameworks calculated to be stable when comparing to known zeolites (450000 as I previously 
mentioned). According to Figure 10 several of the hypothetical frameworks have lower FD than the present record.
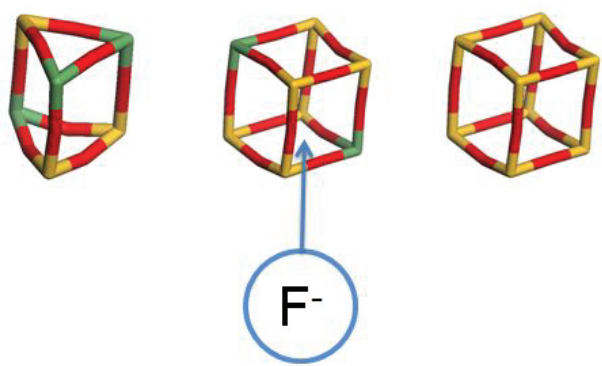

Figure 9: Stabilization of strained structural units by Ge and F. D4R and especially D3R are stabilized by a high loading of Ge. Many of the D4R are typically further stabilized with $F$ as in the case of ITQ-13. [19]
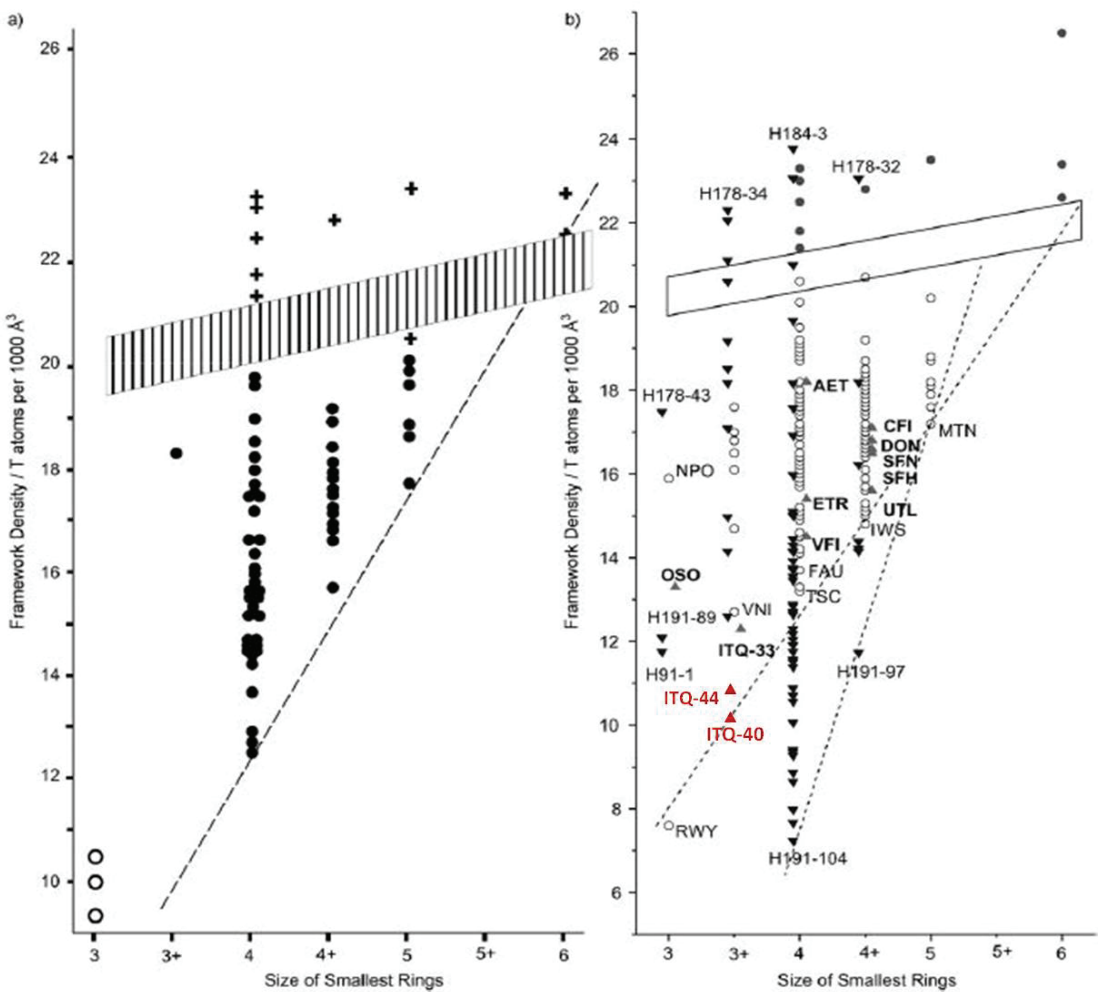

Figure 10: The correlation between framework density (from IZA database [34]) and the size of the smallest ring present in the structure. Symbols represent in a) + dense 
framework, - zeolite, o hypothetical and b) •mineral, $\mathbf{\Delta}$ real and hypothetical $\boldsymbol{\nabla}$ extralarge pore. Dotted lines indicate the linearity for real extra-large pore zeolites and hypothetical extra-large pore zeolites. The parallelograms represent the separation of dense structures and zeolites. Adopted with minor changes from.[7]

\subsection{Materials employed in this work}

\subsubsection{D Zeolites}

Four materials with one dimensional (1D) channel systems have been studied: ZSM-22 (TON), ZSM-23 (MTT), ZSM-48 (MRE*) and EU-1 (EUO) with main parameters given in Table 1. They differ slightly in channel size and the size of the side pockets present as shown in Figure 11. The channel in ZSM-22 is elliptical $(5.7 \times 4.6 \AA)$ with no side pocket while ZSM-23 is straight $(5.2 \times 4.5 \AA)$, but with a small side pocket, giving a teardrop shaped channel. ZSM-48 has nearly symmetrical straight channels with dimension $5.6 \times$ $5.3 \AA$. The channels of EU-1 are straight $(5.4 \times 4.1 \AA)$, but large 12-ring side pockets $(6.8 \times 5.8 \AA$ wide and $8.1 \AA$ deep) are present. As previously reported [35] these materials can be viewed as a series of catalysts with very similar one-dimensional pores with side pockets of sizes increasing in the order ZSM-48 $\leq$ ZSM-22 $<$ ZSM-23 $<$ EU-1.

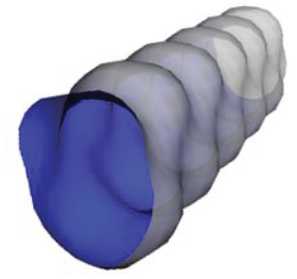

ZSM-22

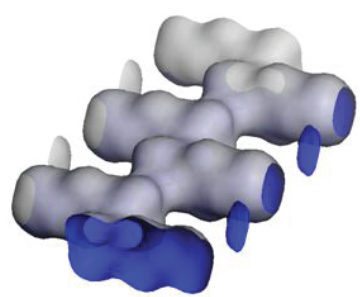

EU-1

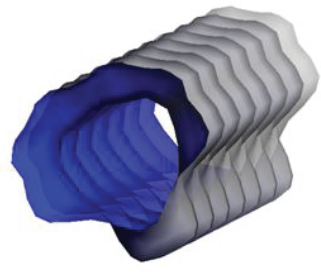

ZSM-23

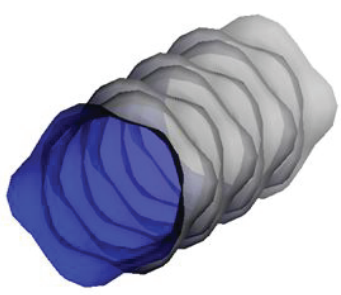

ZSM-48

Figure 11: The channel system of the four $1 D$ zeolites relevant to this work. 
Table 1: Main characteristics of the 1D zeolites ZSM-22, ZSM-23, ZSM-48 and EU-1

\begin{tabular}{|c|c|c|c|c|c|}
\hline \multirow{2}{*}{ Topology } & \multirow{2}{*}{ Structure } & \multicolumn{2}{|c|}{ 10-ring channel system } & \multirow{2}{*}{ Max. diameter $^{\mathrm{a}}$} \\
\cline { 3 - 5 } & & Size & Shape & Side pocket & \\
\hline TON & ZSM-22 & $5.7 \times 4.6 \AA$ & Elliptical & None & $5.71 \AA$ \\
\hline MTT & ZSM-23 & $5.2 \times 4.5 \AA$ & Teardrop & Very small & $6.19 \AA$ \\
\hline MRE* & ZSM-48 & $5.6 \times 5.3 \AA$ & Cylindrical & None & $6.36 \AA$ \\
\hline EUO & EU-1 & $5.4 \times 4.1 \AA$ & Zig-zag & $6.8 \times 5.8 \times 8.1 \AA$ & $7.00 \AA$ \\
\hline
\end{tabular}

*: disordered structure, $\mathrm{a}=$ max. diameter of a sphere that can be included inside the largest cavity/channel.[34]

\subsubsection{D Zeolites}

ZSM-57 (MFS), with key parameters described in Table 2, is the only zeolite with a two dimensional (2D) channel system studied in the present work.[36] One 10-ring channel with dimension $5.1 \times 5.4 \AA$ goes along [100] and intersect another 8-ring channel with dimension $3.3 \times 4.8 \AA$ along [010]. The latter channel is slightly alternating up and down in plane and thus it can be described as slightly zig-zagged. In this structure the largest diameter of a sphere which could be occluded in the channel is $6.81 \AA$.

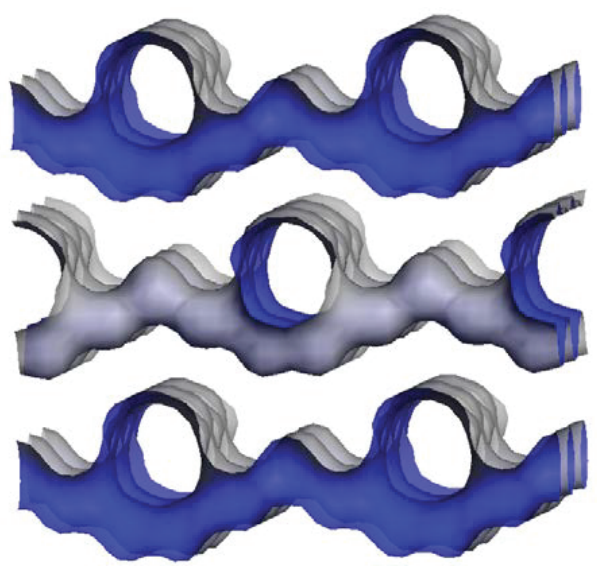

Figure 12: The channel system of ZSM-57 consists of one 10-ring and one 8ring.

Table 2: Main characteristics of the 2D zeolite ZSM-57

\begin{tabular}{|c|c|c|c|c|}
\hline \multirow{2}{*}{ Topology } & Structure & \multicolumn{3}{|c|}{ Channel system } \\
\cline { 3 - 5 } & & Size 10-ring & Size 8-ring & Max. diameter $^{\mathrm{a}}$ \\
\hline MFS & ZSM-57 & $5.1 \times 5.4 \AA[100]$ & $3.3 \times 4.8 \AA[010]$ & $6.81 \AA$ \\
\hline
\end{tabular}

$\mathrm{a}=$ max. diameter of a sphere that can be included inside the largest cavity/channel.[34] 


\subsubsection{D Zeolites}

TNU-9, ITQ-13, ZSM-11 and ZSM-5 are all structures with three-dimensional channel systems with main characteristics given in Table 3. IM-5 is herein counted as a structure having a 3D channel system despite that it consists of a limited 3D system only. Despite the inferior channel dimensions of the ITQ-13 structure compared to ZSM-5 a rather large sphere with diameter of $6.72 \AA$ can fit and this is even larger than the maximum diameter of a sphere able to fit in ZSM-5 (6.36 $\AA$ ). Compared to TNU-9 (8.46 $\AA$ ) and IM-5 (7.34 $\AA$ ) the channel intersection volumes of both ITQ-13 and ZSM-5 are clearly smaller. Thus strictly ranging the materials having three-dimensional channel systems from the smallest to the largest diameter of a sphere the following order results: ZSM-5 $<$ ITQ-13 $<$ IM- $5<$ TNU-9. It should here be emphasized that IM-5 has side pockets which extend the intersection volumes beyond that of the space indicated only by the maximum sphere diameter.

Because of the complexity of the $3 \mathrm{D}$ materials the further descriptions are presented individually for clarity.

Table 3: Main characteristics of the $3 D$ zeolites

\begin{tabular}{|c|c|c|c|c|c|}
\hline \multirow{2}{*}{ Topology } & \multirow{2}{*}{ Structure } & \multicolumn{3}{|c|}{ 10-ring channel system (*9-ring) } \\
\cline { 3 - 5 } & & Size & Size & Size & Max. diameter $^{\mathrm{b}}$ \\
\hline \multirow{2}{*}{ IMF } & IM-5 $^{\mathrm{a}}$ & $\begin{array}{c}5.3 \times 5.4 \AA[100] \\
\text { center } \\
\end{array}$ & $\begin{array}{c}5.1 \times 5.3 \AA[100] \\
\text { side }\end{array}$ & $\begin{array}{c}5.5 \times 5.6 \AA[001] \\
\text { center } \\
4.8 \times 5.4 \AA[001] \\
\text { side }\end{array}$ & $7.34 \AA$ \\
\hline TUN & TNU-9 & $5.5 \times 5.6 \AA[010]$ & $5.1 \times 5.5 \AA[010]$ & $5.4 \times 5.5 \AA[10-1]$ & $8.46 \AA$ \\
\hline ITH & ITQ-13 & $4.0 \times 4.8 \AA[100]^{\mathrm{c}}$ & $4.8 \times 5.1 \AA[010]$ & $4.8 \times 5.3 \AA[001]$ & $6.72 \AA$ \\
\hline MFI & ZSM-5 & $5.1 \times 5.5 \AA[100]$ & $5.3 \times 5.6 \AA[010]^{\mathrm{d}}$ & & $6.36 \AA$ \\
\hline MEL & ZSM-11 & $5.3 \times 5.4 \AA[100]$ & & & $7.72 \AA$ \\
\hline
\end{tabular}

$\mathrm{a}=\mathrm{IM}-5$ contains side pockets which enlarge the channel intersection volume beyond what indicated by a spherical probe

$\mathrm{b}=$ max. diameter of a sphere that can be included inside the largest cavity/channel.[34]

$\mathrm{c}=9$-ring channel

$\mathrm{d}=$ zig-zag channel.

\section{TNU-9 (TUN)}

TNU-9 is one of the most complex zeolite structures ever solved since it is build up by (24) topologically different $\mathrm{T}$ atoms.[37] It is a three-dimensional 10-ring zeolite as two different sized 10-rings along the crystallographic b-axis [010] (5.6 x 5x5 $\AA$ and 5.1 x 5.5 $\AA)$ crosses a slightly sinusoidal channel $(5.5$ x $5.4 \AA)$ along [10-1]. There are no 
connections between the largest channels along [010], only between the large and the small channels along the same axis. The three dimensional channel system, Figure 13, gives two channel intersections.

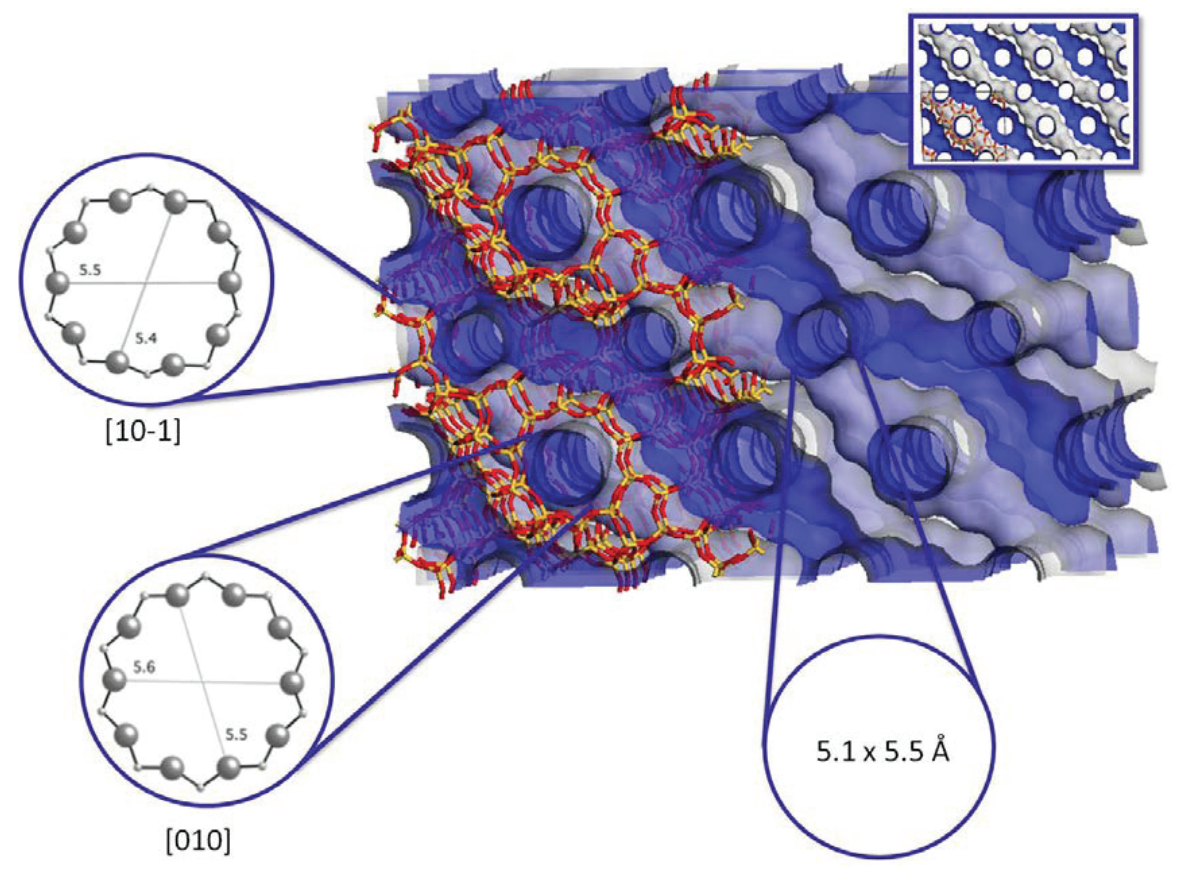

Figure 13: The channel system of TNU-9. Three different 10-rings result in a three dimensional channel system with two different channel intersections.

\section{IM-5 (IMF)}

IM-5 is together with TNU-9 one of the most complex zeolite structures solved.[38] IM-5 does not have a continuous three-dimensional system, but there are regions of three dimensionality separated by walls marked with large red arrows in Figure 14. A twodimensional channel system ([001] $105.5 \times 5.6 \AA \leftrightarrow[100] 105.3 \times 5.4 \AA) * *$ is connected to 10 -rings along [010] $(5.3 \times 5.9 \AA)$ and to a new two-dimensional system ([001] 104.8 $\mathrm{x} 5.4 \AA \leftrightarrow[100] \mathbf{1 0} 5.1 \times 5.3 \AA)^{* *}$ to give the limited three-dimensional channel system.[38] 


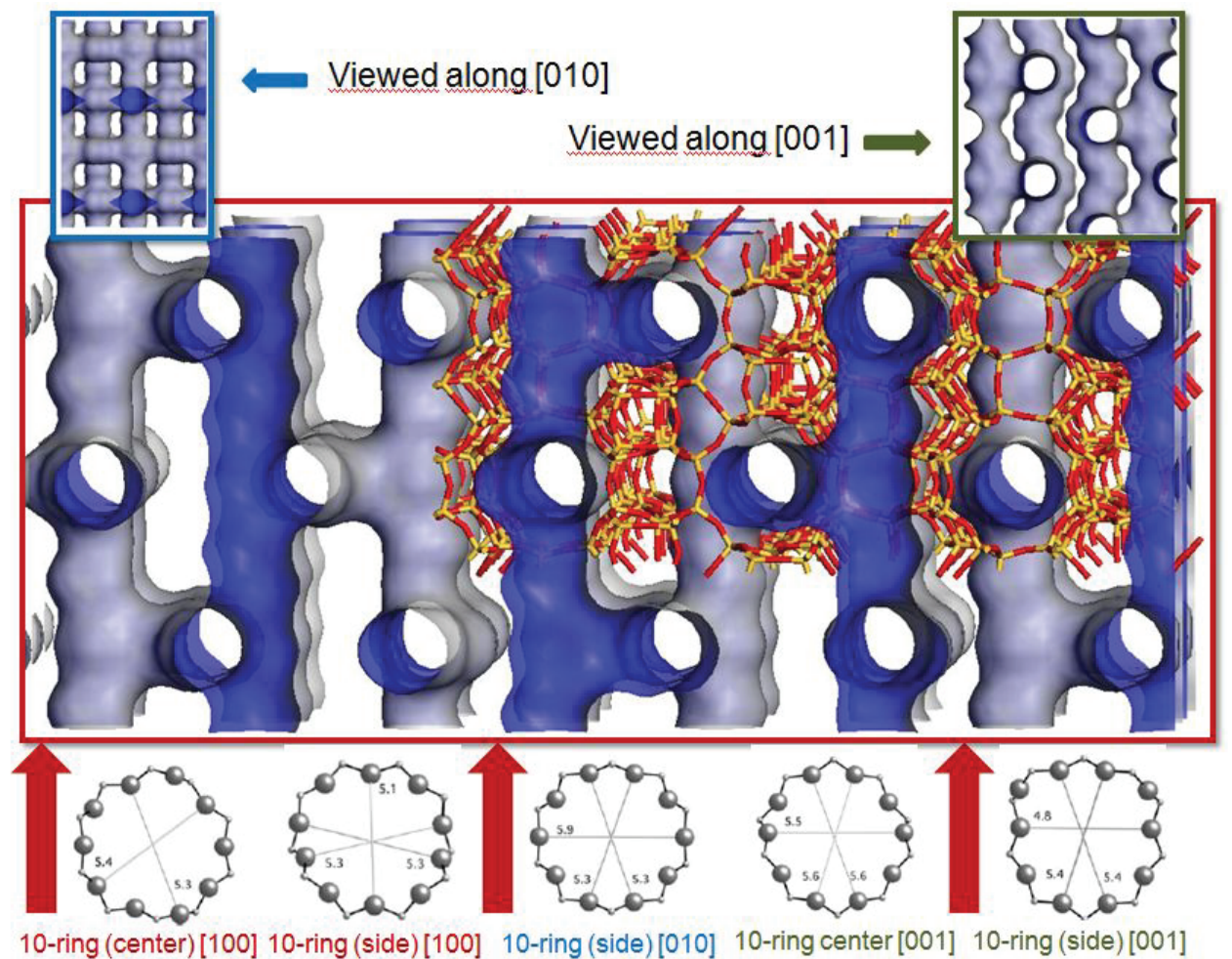

Figure 14: Illustration of the channel system in IM-5, [100] red, [010] blue and [001] green. The red arrows indicate the intermediate region between the limited three dimensional channel systems where there are no connections.

\section{ZSM-5 (MFI)}

The industrially applied ZSM-5 is one of the most familiar and studied zeolites around the globe. ZSM-5 has only two different 10-ring channels, but still it is a three-dimensional system. The reason for this is the slightly alternating zig-zag channel of size $5.3 \times 5.6 \AA$ along [010], alternating up and down along [001], connected to straight channels of size $5.1 \times 5.5 \AA ̊$ along [100]. 

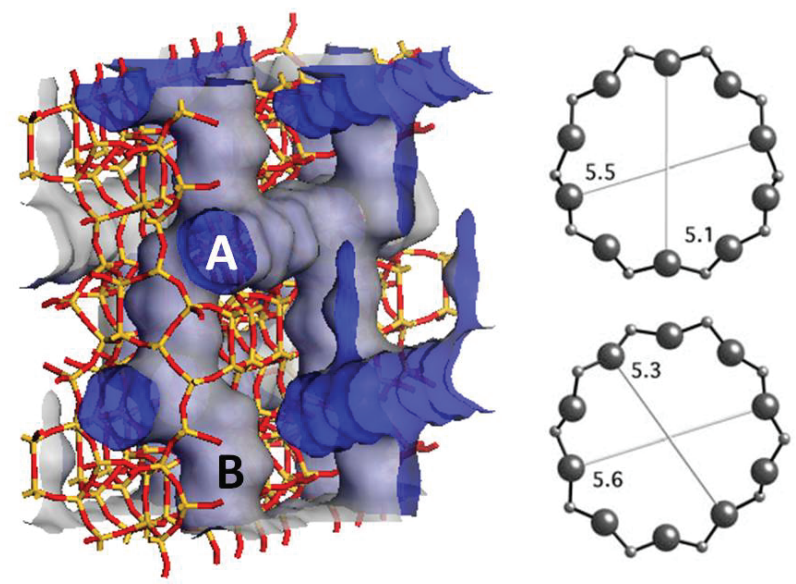

A [100]

B [010]

Sinusoidal

Figure 15: Illustration of the channel system of ZSM-5. Two channels, one sinusoidal $(5.3 \times 5.6 \AA)$ and one straight channel $(5.1 \times 5.5 \AA)$ intersect resulting in a $3 D$ channel system.

\section{ZSM-11 (MEL)}

ZSM-11 has channels with diameter $5.3 \times 5.4 \AA$ in two directions. The intersection between the two perpendicular and slightly shifted straight channels creates the 3D pore system.
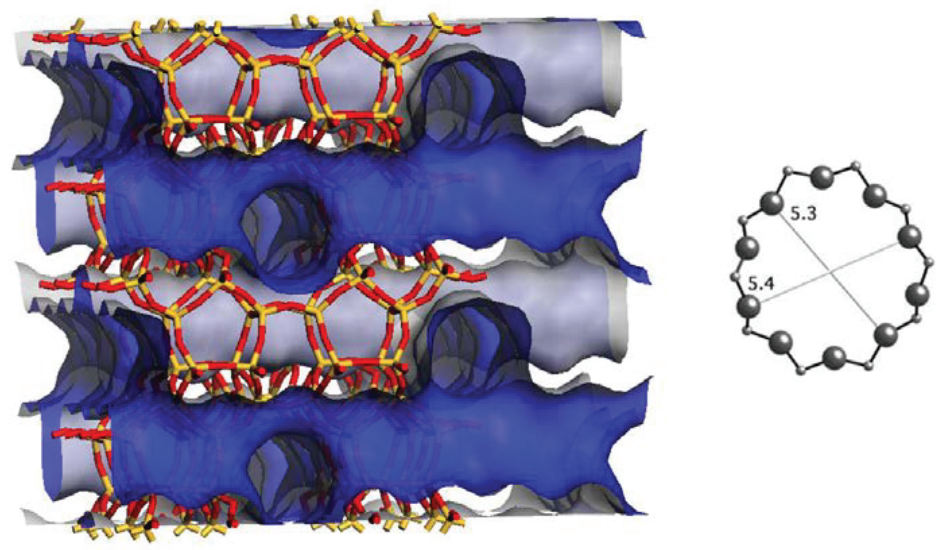

Figure 16: Illustration of the channel system of ZSM-11. Two channels with similar size $(5.3 \times 5.4 \AA$ perpendicular to each other intersect and create a $3 D$ channel system. 
The channels of ZSM-5 and ZSM-11 only slightly differ in size. The lack of a sinusoidal channel in ZSM-11 is one major difference between the two structures.

\section{ITQ-13 (ITH)}

ITQ-13 is a three-dimensional structure as the other structures already mentioned, but the channels are smaller.[39, 40] One straight 10-ring of size 4.8 x $5.1 \AA$ goes along [010] and this is connected to another zigzag 10-ring channel, $4.8 \times 5.3 \AA$ along [001]. Finally, a very interesting feature with ITQ-13 is the presence of a rare small ring containing $9 \mathrm{~T}$ atoms $(4.0$ x $4.8 \AA$ ) crossing the two other channels. The channel system can be viewed in Figure 17.

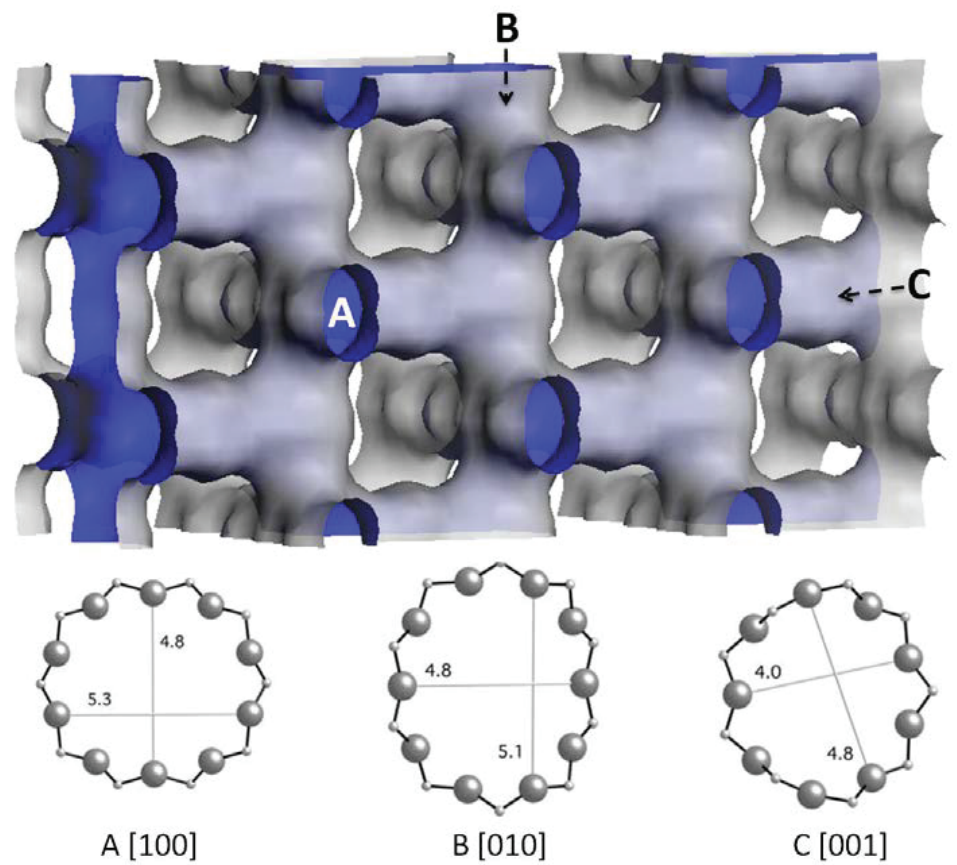

Figure 17: Illustration of the channel system of ITQ-13. 


\subsection{Challenges of zeolites as catalysts}

\section{Shape selectivity is influenced by many parameters}

The selective properties of the catalysts are the fundament of green chemistry as they hinder the formation of byproducts leading to waste by selecting among the possible products with rather similar thermodynamic stability.[1] In zeolite based hydrocarbon conversion catalysis many parameters are known to influence the selectivity. Both the acid site density, type of acid site (Lewis or Brønsted), location and strength of acid sites, particle size and morphology, topology and reaction conditions may influence the selectivity.[41]

Obviously the topology is of main importance in zeolite selective chemistry. When heavy feedstocks are cracked in the oil refinery large pore zeolites (12-ring Zeolite Y) are needed to allow the passage of the large hydrocarbons through the channel apertures. To apply SSZ-13 with 8-ring channels in the same process would be of no interest since large hydrocarbons would be excluded from being selectively cracked. Vice versa it would be of little interest to synthesise small olefins, e.g. ethene, from methanol over Zeolite Y compared to SSZ-13 (or its zeotype analogue SAPO-34).

\section{Complex synthesis systems}

Further complication arises when rather similar zeolites, e.g. all having 10-ring based channels with slight differences in size, is compared under similar reaction conditions (PAPER I). One reason for this is the severe lack of control of the process of zeolite formation. Few methods other than pre and post characterization of zeolite synthesis are available (some exceptions e.g. [42]) due to pressurized systems with harsh environment. In addition time dependent varying solubility and precipitation often lead to situations where more than one parameter is changed at the time (e.g. morphology change as the $\mathrm{Si} / \mathrm{Al}$ ratio is varied). Despite that the channel dimensions are rather similar it calls for synthesis and characterization methods in which the $\mathrm{Si} / \mathrm{Al}$ ratio, location of acid sites and acid strength, defects, morphology control etc. are kept rather unaltered to avoid effects of other parameters in the study. In other words if the aim is to elucidate the effect of slight differences in channel sizes it is difficult to do so if one structure contains significantly more $\mathrm{Al}$ than the other one because this will affect both deactivation rate, the possibility 
to do chemistry interacting with more acid sites (cracking, isomerization etc.) and the interaction among the adsorbed species within the pores changing electron flows. Despite many challenges with respect to zeolite synthesis (close to) one parameter studies exists. One example is a broad variation of $\mathrm{Si} / \mathrm{Al}$ ratio without change in morphology as shown for SSZ-13.[43]

\section{Location of the acid site}

In general it is plausible to explain the position of the acid site in relation to the positive charge of the template, especially in synthesis free from inorganic cations where the negative charge from the Al exclusively is compensated by template ions. Ferrierite (FER) is one example in which the acid site location can be controlled by a proper choice of the template or template mixtures.[44] By partial or full replacement of pyrrolidine with tetramethylammonium ions $\left(\mathrm{TMA}^{+}\right)$protons could be moved from low accessible cage locations to the more open surroundings accessible from the 10-ring channel.[44] However on this fundamental level it requires thoroughly studied synthesis systems and it is not easy to transfer the knowledge directly to other structures because other templates with different $\mathrm{C} / \mathrm{N}^{+}$, ratio, size, rigidity and shape is required. The clear insight on how to place the acid sites just by changing synthesis procedures is far from known and is often still based on trial and error and educated guesses.

\section{Controlled morphology}

Morphology induced selectivity is one factor in designing proper materials in. The reason for this is the potential of changing the crystal dimensions relative to the orientation of the channel systems in the zeolites. If a zeolite consists of e.g, one 10-ring and one 8-ring, e.g. perpendicular to each other, it would be possible to facilitate product formation from one of these channels by increasing the length of the crystal in specific direction (increasing the length of specific channels). It may be possible to increase the contribution from the 8-rings by increasing the length of the crystal along this channel, or opposite, reduce the contribution from the 8-ring by reducing the length along the similar direction. In this concept the product formation is linked to diffusion in the different channels. For the concept of morphology induced shape selectivity see e.g. unpublished work "Morphology induced shape selectivity in zeolite catalysis".[45] 
One major challenge in zeolite synthesis has been to control morphology such that two different zeolites after synthesis result in similar crystal shape and size. Even for one topology several morphologies may appear as the composition of the crystals change. Recently, Choi et al. [46] introduced a new method for the synthesis of nano-structured, but fully crystalline zeolites with the MFI structure using multi-quaternary ammonium surfactants. This combined surfactant-templating method allowed sheets to be formed because the template side allows the zeolite to form while the lipophilic end keeps the sheets apart from each other. Further investigations by increasing the number of amine groups to expand the length of the template side of the surfactant made it possible to even control the thickness of the sheet. Such methods have a potential to be applied to many structures and it opens up new ways to control a rather chaotic synthesis system. Again the dimensions can be designed to tune both selectivity and diffusion rates. Still this field is rather new and requires advanced synthesis procedures

\section{Diffusion limitations and deactivation}

Despite the fact that the presence of micropores in zeolites, with aperture diameters below $1 \mathrm{~nm}$, gives shape selective properties, it imposes on the other hand diffusion limitations that adversely affect catalytic performance. Trapped carbon species can grow larger by addition of feed molecules and result in pore blocking hindering the molecular transport of reactants and products. This calls for frequent regeneration by burning off the coke in the presence of air to reopen the blocked pores. This increases the cost of industrial investment and production, and regeneration is associated with $\mathrm{CO}_{2}$ emissions. Hence, enhancing the transport capacity in zeolite catalysts has been one of the major new issues in zeolite science during the last decade.[47] A typical way to solve such issues has been to make small particles. On the other hand small particles may lose shape selective properties. Thus, an important challenge within the field of zeolite based catalysis is to combine ideal selectivity with long lifetime. The parameters affecting selectivity is often involved in the deactivation pattern of the zeolites in hydrocarbon conversion.[41]

Simple ways to improve diffusion capacities has been developed. One such way to improve diffusion in zeolites has been to expose structures to destructive methods like alkaline treatment with $\mathrm{NaOH}$ to obtain mesopores acting as molecular highways.[48, 49] This leads to partly amorphisation and gives properties intermediate of MCM-41 type 
mesoporous materials and zeolites. A more sophisticated method is to combine surfactants with $\mathrm{NaOH}$ to obtain controlled sizes (dependent on the micelle sizes) of mesopores with reduced material loss.[50, 51] Both $\mathrm{NaOH}$ methods, desilication, and surfactant methods have been studied in this thesis (PAPER IV) and will therefore be presented.

\subsection{Solving bottlenecks}

\subsubsection{Desilication}

Desilication is a post synthesis treatment based on the attack on the Si (typically a silanol group related to defective sites) by a base to weaken the $\mathrm{Si}-\mathrm{O}$ bonds in siloxane (ESi-O$\mathrm{Si} \equiv$ ) or $\equiv \mathrm{Si}-\mathrm{O}-\mathrm{Al} \equiv$. In the original work hot $\mathrm{Na}_{2} \mathrm{CO}_{3}$ solutions selectively removed Siatoms from the framework of ZSM-5, Zeolite Y and to some extent from the Al rich Zeolite X. [52] More efficient desilication was later observed when $\mathrm{pH}$ was adjusted adding $\mathrm{NaOH}$ in addition to the $\mathrm{Na}_{2} \mathrm{CO}_{3}$ as hot solutions over the zeolites.[53] Today, $\mathrm{NaOH}$ is often applied for desilication processes. $\mathrm{OH}^{-}$will attach to the $\mathrm{Si}$ and make $\equiv \mathrm{Si}$ $\mathrm{OH}$ bonds. Further attack on near by Si atoms will lead to dissolution of parts of the zeolite as fragments lose the connection to the framework as shown in Figure 18.

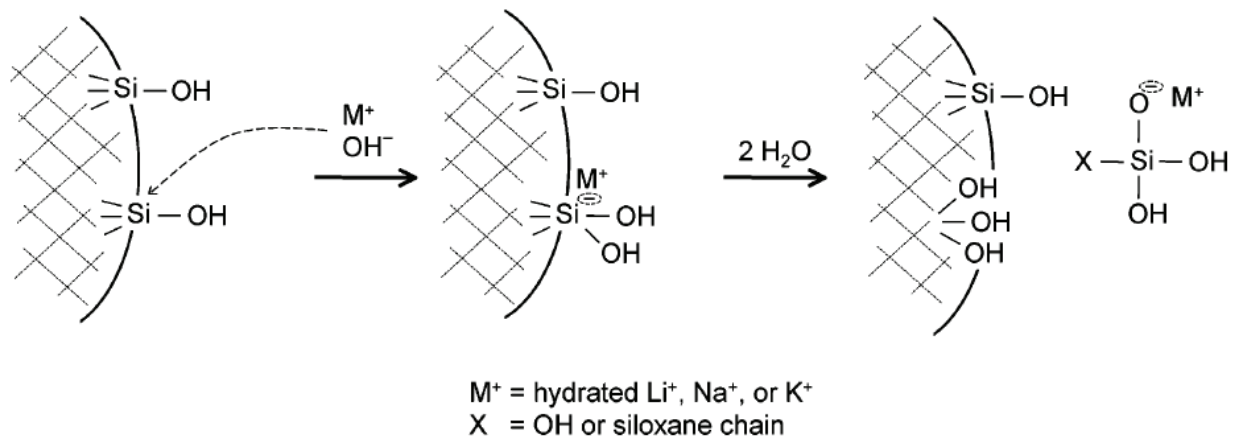

Figure 18: Concept of mesopore formation in alkaline medium. Adopted from [54].

The most dominant parameter influencing the mesopore formation is normally the treatment conditions (concentration of the $\mathrm{NaOH}$ solution, temperature and time). 
However other parameters influence the formation of these pores and their shape. Topology: The T-atoms in zeolites are mainly exposed to either the external surface or to the inner surface of the crystal. New passages will open when parts of the walls are dissolved because on each side of the walls there are channels and cages. Therefore the choice of topology may give implications on the shape and size of the pores obtained after alkaline treatment, e.g. when walls between a small channel and a large cage are dissolved instead of walls separating two large cages. Si/Al ratio and acid site location: Also Al will affect the mesopore formation because Al introduces a net negative charge and such positions are less vulnerable to attack by Lewis bases because of the repulsion between negatively charged $\mathrm{AlO}_{4}{ }^{-}$tetrahedra and the $\mathrm{OH}^{-}$.[55] Clearly the closest $\mathrm{Si}$ is stabilized by this negative charge introduced by the Al and this hinders the breakage of Si-O-Al bonds. The less efficient incorporation of mesopores shown for Zeolite $\mathrm{X}$ compared to Zeolite Y and ZSM-5 can also likely be attributed to the higher Al content of Zeolite X. Detailed studies on MFI type zeolites varying the $\mathrm{Si} / \mathrm{Al}$ ratio showed different degree of dissolution.[56] When $\mathrm{Si} / \mathrm{Al} \leq 15$ the incorporation of mesopores was not efficient and for $\mathrm{Si} / \mathrm{Al} \geq 200$ excessive dissolution took place. This led to the conclusion that the optimal $\mathrm{Si} / \mathrm{Al}$ ratio for mesopore incorporation was in the range of $\sim 25-50$ as shown in Figure 19.[56] It should also be emphasized that the stability of the Al in the framework may differ and thus be crucial to the mesopore formation.[57] For instance the low stability of $\mathrm{Al}$ in four-membered rings in Beta led to removal of acid sites, which is not wanted for its catalytic activity, upon desilication.[58] Defects: In a study of MFI type zeolite particles with different numbers of intergrowth sites or defects the $\mathrm{Si} / \mathrm{Al}$ ratio seemed to have minor effect on desilication in cases where the number of defective sites was high.[59] A preferred range of $\mathrm{Si} / \mathrm{Al}$ ratios was $\sim 20-50$ with respect to desilication of zeolites with rather few defects and intergrowths in accordance to [56] (see Figure 20). However, in crystals with no or little intergrowths and defects the $\mathrm{Si} / \mathrm{Al}$ ratio of $\sim 20-50$ was required to improve the transport capacities in the zeolite. 


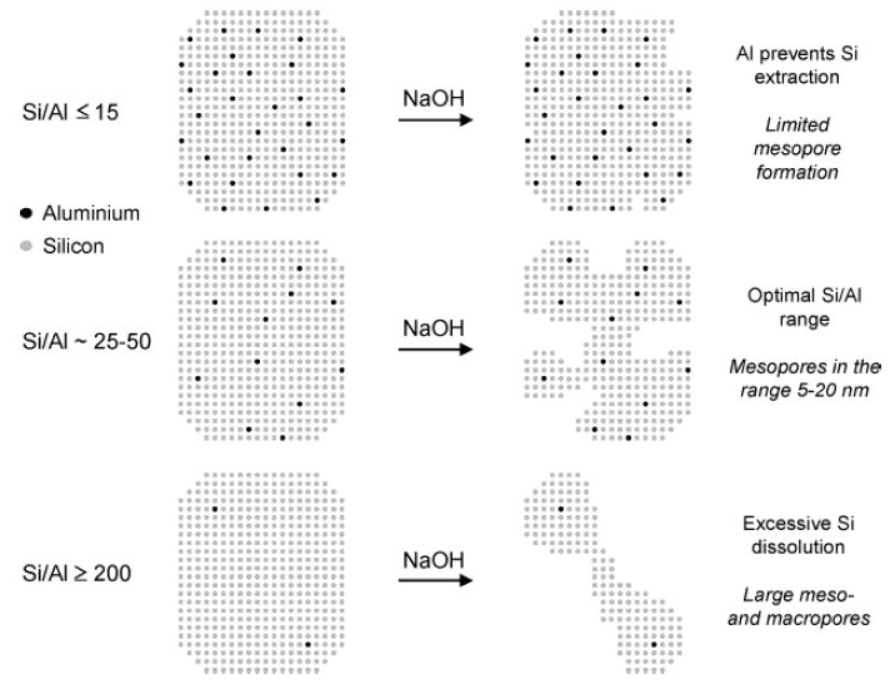

Figure 19: The effect of the Si/Al ratio on the desilication process on MFI type zeolites. Preferential S/Al ratios for obtaining controlled mesopores was in the range of $\sim 25$ 50.[56]

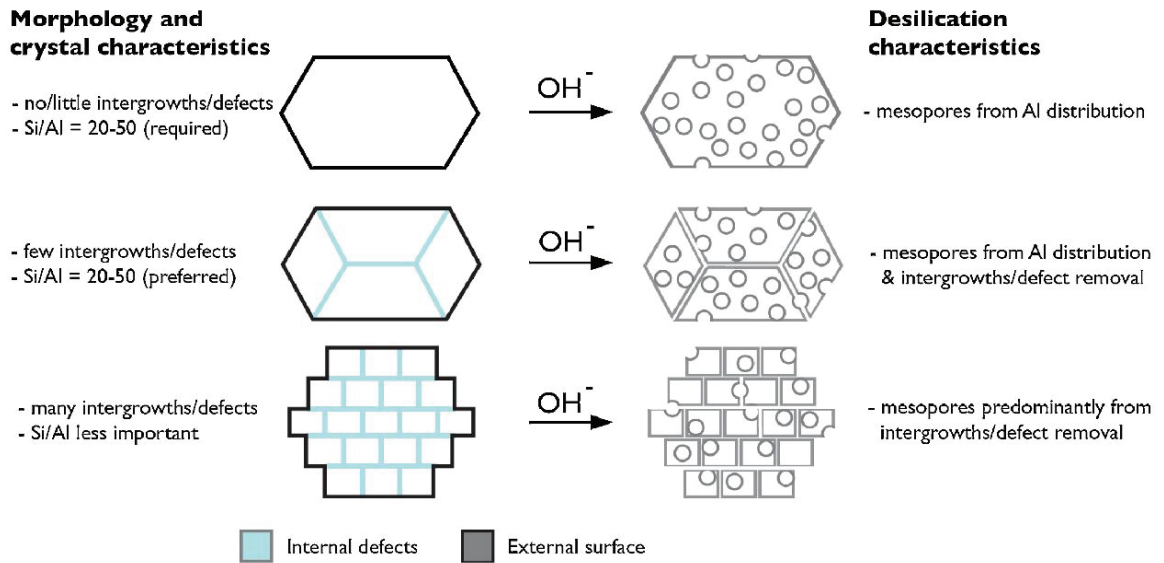

Figure 20: Idealized mesopore formation mechanism in zeolites as a result of defect and intergrowth density and Si/Al ratio.[59]

Morphology: Verboekend et al. [60] studied ZSM-22 and FER type zeolites and found only limited development of mesopore surface area and low desilication efficiency (desilication efficiency $=\Delta S_{\text {Meso }} / \Delta$ (weight loss) where $\Delta S_{\text {Meso }}=$ mesopore surface of the alkaline treated minus the mesopore surface of the parent material) for these materials. 
They suggested that the needle shaped crystals of ZSM-22 and the plate like crystals of ferrierite were less favorable for desilication. In the order of decreasing size of the smallest dimension of the crystals (ZSM-5 $>$ ITQ-4 $>$ ferrierite $>$ ZSM-22) the desilication efficiency was found to dramatically decrease. They concluded that efficient desilication ( $>4 \mathrm{~m}^{2} / \mathrm{g} \%$, change in mesopore surface per percent of weight loss) could be carried out when the crystals were at least $200 \mathrm{~nm}$ in three dimensions.[61] The low desilication efficiency of mordenite has also been attributed to small crystal dimensions.[62] Many zeolites with unidirectional channel systems and few pore mouths have needle shaped morphologies as the crystals tend to grow along the only channel. In such cases one dimension is small and may hinder efficient desilication or lead to fragmented crystals.

In summary, desilication is dependent on defective sites, morphology, $\mathrm{Si} / \mathrm{Al}$ ratio and topology as well as temperature, concentration and time in which the $\mathrm{NaOH}$ is allowed to etch the crystal, and offers a cheap an easy way to improve molecular transport capacities in zeolites since wider pores are introduced in addition to the micropores.

\subsubsection{Combined alkaline and surfactant treatment}

Despite that desilication offers a simple and inexpensive method for introducing mesopores such treatment is often not necessarily selective to the pore size formed. Recent studies on combining $\mathrm{NaOH}$ and surfactants have shown to give controlled mesopores in ZSM-5 [50, 51] and in FER-type zeolites.[63] Surfactants (e.g. cetyltrimethylammonium bromide - CTAB) organized in micelles with polar ends pointing towards the solution may connect to dissolved silicate species and recrystallize/re-assemble with the zeolites (eventually by precipitation). This process takes place in heated solutions (both hydrothermal and lower temperatures than the boiling point of the water), and literally by growing in to the zeolite it leaves pore sizes defined by the micelle size and shape upon calcination. Cetyltrimethylammonium bromide with $16 \mathrm{C}$-atoms in the surfactant chain typically gave mesopores in the range of 3-10 nm, but by varying the chain length the micelle size and thus the mesopore size can be further tuned to smaller or larger sizes or size distributions as shown in [51]. Such treatments are often accompanied by less loss of materials compared to pure desilication. 


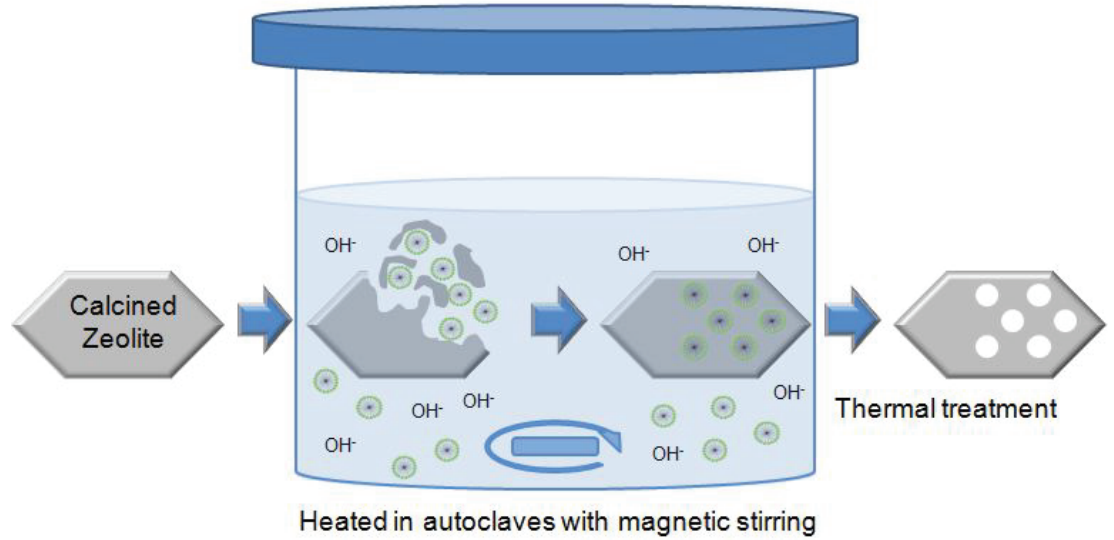

Figure 21: Illustration of the surfactant assisted recrystallization/ re-assembly of a zeolite particle attacked by $\mathrm{NaOH}$.

\subsubsection{The sugar method}

The sugar method, based on in situ decomposition of sucrose as hard template for hierarchical zeolite, was developed by Kustova et al. [64]. The mentioned work focused on ZSM-5 and ZSM-11, but the experimental approach is applicable to other materials. Porous silica gel was impregnated with aqueous sugar solutions to incipient wetness. This was then (dried at room temperature over night and) pyrolysed in the absence of air to carbonize the sugar. Hence in the silica pores carbon could be trapped and the silicacarbon composite was then used as a silica source in the zeolite synthesis which allowed crystal growth occluding the carbon during crystallization. Upon calcination the carbon disappeared and left a pore with size in the mesopore range.

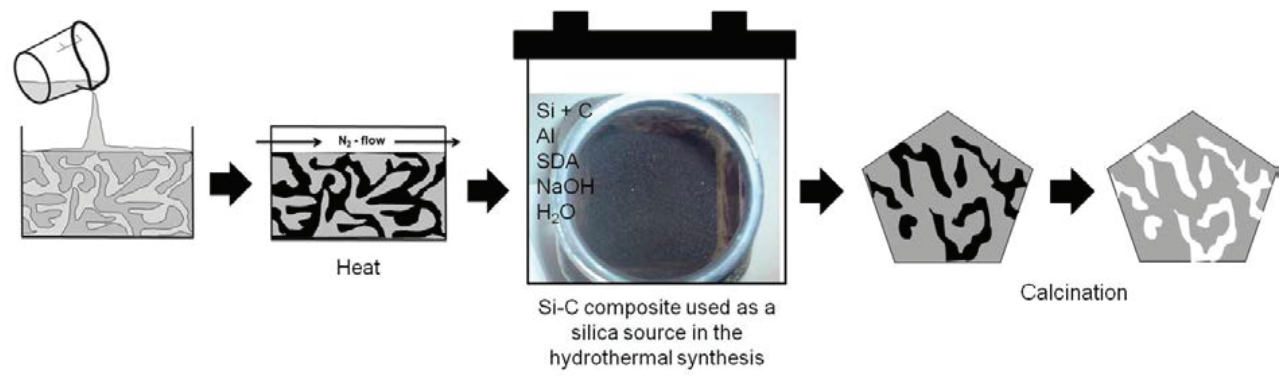

Figure 22: The principle steps in the sugar method. Porous silica gel is impregnated (to incipient wetness) with aqueous sucrose solution. The mixture is 
heated in inert gas to carbonize the sugar. The resulting Si-C composite is used as a silica source which allows crystallization to zeolites occluding the carbon. The final step is calcination of the zeolites to open the pores with size dependent on the carbon inside.

\subsubsection{The carbon black method}

Several (porous) carbon sources like carbon nanotubes, nanofibers and carbon black can be used to make mesoporous zeolites. The carbon templating method has been developed from zeolite growth on hollow carbon nanofibers with several micrometers in diameter leading to intercrystalline meso- and macropores, to the possibility to incorporate controlled intracrystalline mesopores with other carbon sources like carbon black.[65] The latter processes developed by Haldor Topsøe leading to intracrystalline mesopores involves impregnation with stepwise addition of reactants in solutions of a carbon source. e.g. carbon black (e.g. BP2000) with zeolite precursors. The stepwise procedure involves impregnation, homogenization and solvent evaporation. An example of a typical sequence is given in Figure 23.

\section{Day 1:}

One reagent solution impregnated to Carbon Black

Homogenised by manually stirring (no big lumps)

Solvent evaporated (normally over night)

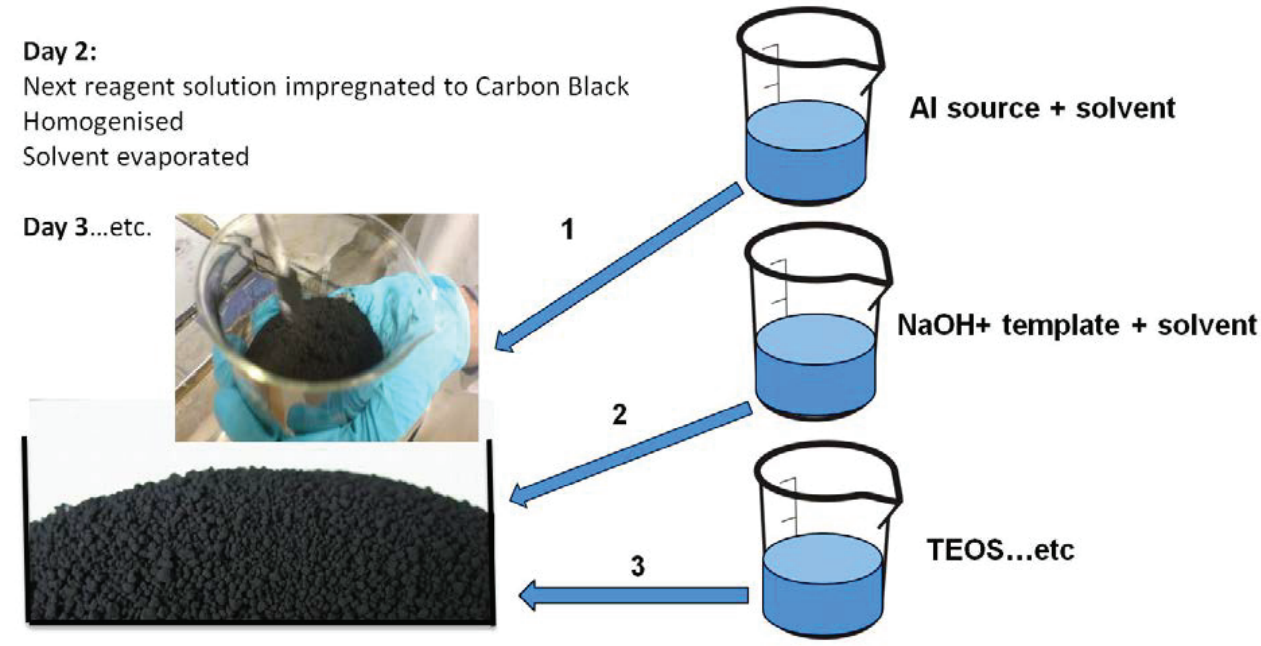

Figure 23: The principle steps in the Carbon Black method. Each step involves impregnation, homogenization and solvent evaporation. 
The stepwise addition of precursor solutions is followed by hydrothermal treatment. In this way the amorphous reactants crystallizes around the carbon nanoparticles which can be subsequently burned off in presence of air and thus leave mesopores with size defined by the size of the carbon nanoparticles. If the particles grow too big the carbon particles may be occluded inside the crystal leading to less efficient ink bottle type mesopores. Eventually particles can also grow in between the carbon particles leading to confined space synthesis. In summary secondary templating with carbon sources other than the expensive carbon nanotubes is one promising method of introducing mesopores in zeolites. The principle route is shown in Figure 24.

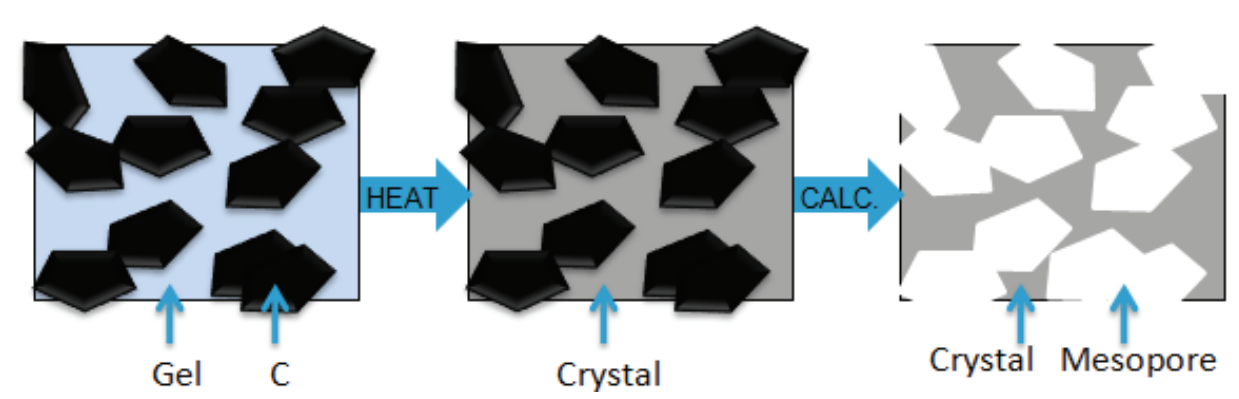

Figure 24: The principle route with Carbon Black. The porous carbon is impregnated in a several step procedure or eventually in one pot. The samples are hydrothermally treated in which the precursor mixture crystallizes around the carbon. Upon calcination in presence of oxygen pores defined by the carbon particle is obtained.

\subsubsection{The effect of introducing mesopores}

In microporous zeolites the size of pores has a similar order of magnitude as the size of the molecules involved. In such situations the diffusion does not follow the classical diffusion theories. As the transport to and from the active sites is slow, diffusion limitations may severely reduce the catalyst efficiency, as typically only the outer parts of the crystal are utilized for reactions and many acid sites are therefore not even reached by reactants or other species involved. Compared to meso- and macropores with diffusion typically observed within the range of $10^{-8}-10^{-4} \mathrm{~m}^{2} / \mathrm{s}$, micropores show slower diffusion typically within $10^{-20}-10^{-8} \mathrm{~m}^{2} / \mathrm{s}$. [66] In cases where diffusion is slower than the reactions taking place the diffusion may be responsible for the overall rate determining step. The methods described above are some of many possible ways to overcome the diffusion 
barriers in zeolites

Introduction of mesopores have shown to greatly improve the catalyst performance, resulting in improved lifetimes and a higher utilization of the crystal. Kim et al. [67] investigated the effect of mesoporosity against the deactivation of MFI zeolite with different degrees of mesoporosity (obtained by post-synthetic desilication, dry-gel conversion in nanocarbon template, and hydrothermal synthesis with the addition of organosilane surfactants) in the MTH process. They found an increased lifetime by more than three times after mesopore introduction, with roughly a linear correlation between mesoporosity and catalyst lifetime. One possible explanation to the improved lifetime was the short diffusion lengths facilitating diffusion of coke precursors from the micropores to the external surface or mesopore walls. Thus deactivation occurred as a result of internal coke formation in the micropores. Interestingly dealumination of the external crystal surface caused no clear difference in coke formation between external and internal locations. However when the internal Al was removed the lifetime clearly increased. As a final conclusion the generation of mesopores did not change the catalyst activity which implies that the mesoporosity could be maximized to increase catalyst efficiency (in other words the zeolite walls could be minimized).

In In the review of Pèrez-Ramirez [57] the relation between the Thiele modulus $(\phi)$ and the effictiveness factor $(\eta)$ was presented. A low Thiele modulus results in a low utilization of the catalyst. Hence in these cases the concentration throughout the catalyst is much lower than the surface concentration (c/ $\mathrm{c}_{\mathrm{s}}$ concentration approaches 0$)$. In cases with almost no diffusion limitations the surface concentration is almost similar to the concentration throughout the catalyst and hence $\mathrm{c} / \mathrm{c}_{\mathrm{s}}$ ratio approaches 1 . In cases where the diffusion lengths (L) is small the $\mathrm{c} / \mathrm{c}_{\mathrm{s}}$ also approaches 1 . This means that $\mathrm{L}$ increases towards the inner of the catalyst particle. Hence reducing the crystal size leads to improved utilization of the crystals. The other possibility is to introduce molecular transport highways (mesopores) in the crystal and hence reduce $\mathrm{D}_{\text {eff. }}$. To introduce efficient mesopores the mesopore should connect the inner parts of the crystal to the external parts shown in Figure $25 \mathrm{~g}$. 

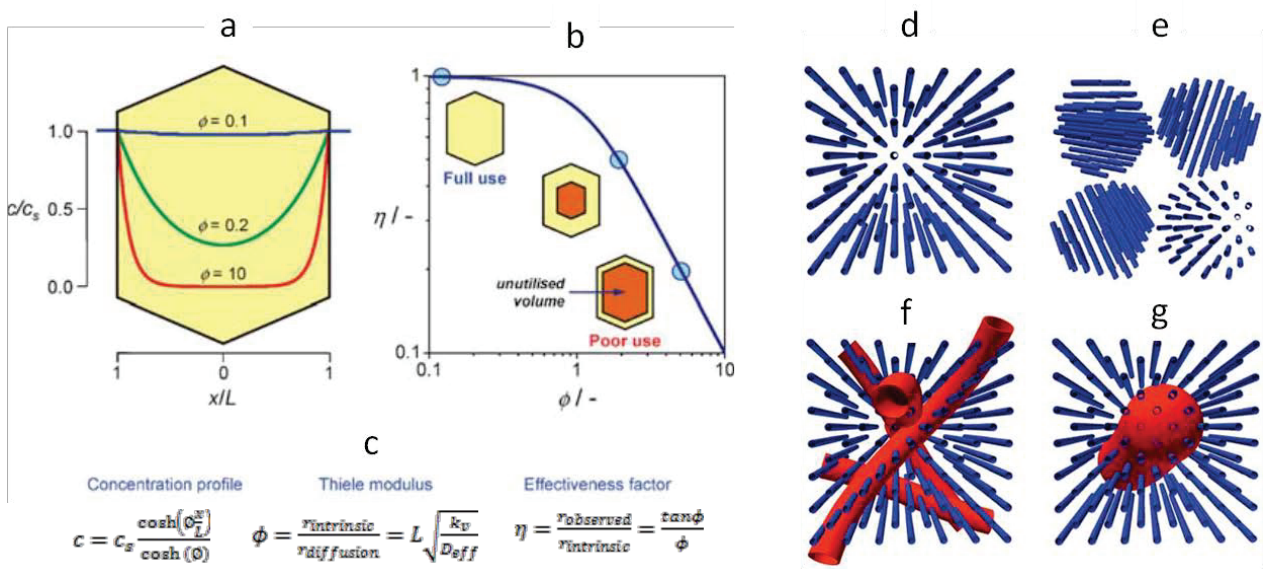

Figure 25: A modified picture from [57]. A-c deals with concentration profiles and crystal utilization while $d-g$ deals with the relative position of micropores (blue) and mesopores $(r e d)$. The figures $a$ and $b$ were derived assuming steady-state diffusion and reaction, slab model, first-order irreversible reaction, and isothermal conditions. Baur and Krishna [68] addressed the applicability of classical definitions of Thiele modulus and effectiveness factor for zeolites.

In cases where the mesopores are trapped inside the crystal as in $h$ (no exits to the outer parts) it is not obvious that such types of mesopores are efficient in improving the transport capacity in the zeolites. Also in the case of intercrystalline mesopores the transport capacity within each crystal is not improved. 


\section{Methanol To Hydrocarbons (MTH)}

As the living standards increase in many countries the demand for energy and petrochemical products has increased. Traditionally oil refineries have been the principle supply for many of the needed products such as fuels (e.g. gasoline and diesel) for the transport sector. Still large oil fields are found such as the recent discovery in Brazil, but it is forecasted that the overall crude oil reservoirs will continue to deplete. Alternative ways to produce high value products from other $\mathrm{C}$ sources (e.g. coal, natural gas, wood/plants, waste from restaurants and farming and domestic waste etc.) than oil exist and it is expected that such sources will increase its importance in the future.[69] The completion of Shell's "Pearl" based on Fischer Tropsch technology utilizing natural gas with a peak production of $\sim 45,3$ million $\mathrm{m}^{3} /$ day of gas resulting in: 140000 barrels/day of gas-to-liquids products (2 trains) and 120000 barrels/day of natural gas liquids and ethane shows the importance of the alternative C sources.[70] Another way of utilizing alternative $\mathrm{C}$ sources is the Methanol To Hydrocarbons (MTH) process which represents the final step in a reaction series leading to valuable end products such as gasoline and plastic monomers. This leaves methanol as an important reaction intermediate in alternative $\mathrm{C}$ source conversion. The latter process is in focus in this thesis and therefore an overview of the historical developments and the MTH mechanism will be given in the following. The related contribution from this thesis to the MTH reaction is given in "summary of results" and in the respective papers attached.

\subsection{Historical development}

The possibility to convert methanol into hydrocarbons over a protonated zeolite catalyst has been known since the late 1970's when researchers at Mobil searched for methods to synthesis high octane gasoline from methanol and isobutane over ZSM-5.[71] To their surprise a wide range of products formed, not only the highly branched alkanes expected from methanol addition to isobutane. When the isobutane feed was cut the catalyst continued to produce hydrocarbons over the catalyst. The conclusion was that the observed products were formed from methanol itself. The high oil price partly caused by the Yom Kippur war and the revolution in Iran resulted in commercial interest of the 
MTH reaction. Mobil built a factory producing gasoline from methanol in 1985, but when the oil price dropped after start up the production was closed down and switched to methanol.[41] The MTH part was later removed from the plant. Today the oil price is again high, which can be seen from the historical development of the oil price and the MTH process has regained attention.

The products obtained in the MTH reaction heavily depends on the type of zeolite (topology). The (14500 barrels per day) Methanol To Gasoline (MTG) factory Mobil built after their discovery was based on a medium pored zeolite ZSM-5, a zeolite with three dimensional (3D) pore system based on 10-ring channels of size $5.4 \times 5.6$ and $5.3 \times 5.5 \AA$ to give products of mainly $\mathrm{C}_{5}+$ hydrocarbons. $[41,72,73]$ The archetype ZSM-5 catalyst is applied also in the Topsøe Integrated GAsoline Synthesis (TIGAS), an improved version of MTG with merged DME and methanol synthesis.[74]

The small pored SAPO-34, a zeotype with a 3D cage structure with window size $3.8 \times 3.8 \AA$ is used in UOP/Hydro Methanol To Olefins (MTO) synthesis targeting the plastic raw materials (mainly ethene and propene) market.[75] Another important industrial process is the Methanol To Propene (MTP) developed by Lurgi.[76] Also here ZSM-5 works as a catalyst, although the conditions are optimized for propene production by lowering the pressure and increasing the temperature in the reactor. Heavier compounds are recycled in the process.

Despite the turbulent financial times the oil price has kept its high levels. Risk of falling gas prices due to the US shale gas revolution and flexibility with respect to $\mathrm{C}$ source (e.g. coal relevant for China among others) should give possibilities to utilize the technology. Since 2008 the number of demonstration plants and licensing of the MTH technology, especially MTO based on methanol from coal has boosted, but also MTG and MTP technology has been applied. An overview of the development in MTH application is given in Table 4 .

Table 4: Development in MTH licensing, demonstration and commercial scale plants.

\begin{tabular}{|c|c|c|c|}
\hline Year & Developed by & Process & Status \\
\hline $1981-1984$ & Mobil & MTG & Demonstrated on a 4 b/d plant in Paulsboro,NJ, USA \\
\hline
\end{tabular}




\begin{tabular}{|c|c|c|c|}
\hline 1981-1984 & Mobil & MTG & $\begin{array}{l}\text { Demonstrated on a } 100 \mathrm{~b} / \mathrm{d} \text { plant in Wesseling, } \\
\text { Germany }\end{array}$ \\
\hline 1985 & Mobil & MTG & Commercialized in New Zealand (14500 b/d) \\
\hline 1980s & Haldor Topseøe & TIGAS $^{\mathrm{b}}$ & $\begin{array}{l}\text { A demonstration plant developed based on ZSM-5 (1 } \\
\text { t/d).[74] }\end{array}$ \\
\hline 2008 & UOP & MTO & $\begin{array}{l}\text { UOP agreed with Methanol Ltd., a subsidiary of } \\
\text { EuroChem. A commercial-scale plant was expected } \\
\text { to come on stream in 2012. Project delayed due to } \\
\text { financing. [77] }\end{array}$ \\
\hline 2009 & Shanxi Coal Institute & MTG & $\begin{array}{l}\text { A demonstration plant brought on stream in Shanxi } \\
\text { province, China }(100 \mathrm{kt} / \mathrm{y})[41]\end{array}$ \\
\hline 2009 & $\begin{array}{l}\text { UOP/INEOS and } \\
\text { Total OCP }\end{array}$ & MTO & $\begin{array}{l}\text { A semi-commercial demonstration unit built in } \\
\text { Feluy, Belgium }(10 \mathrm{t} / \mathrm{d})\end{array}$ \\
\hline 2010 & CAC Chemnisz & STF & $\begin{array}{l}\text { Currently in a demonstration phase syngas-to-fuel } \\
\text { unit, developed in Germany. }\end{array}$ \\
\hline 2010 & Shanxi Coal Institute & MTG & $\begin{array}{l}\text { Currently in a demonstration phase, developed in } \\
\text { China. }\end{array}$ \\
\hline 2010 & Haldor Topsøe & TIGAS & $\begin{array}{l}\text { Currently being demonstrated in Des Plaines, USA, } \\
\text { where a wood gasifier is running. [78] }\end{array}$ \\
\hline 2010 & $\begin{array}{l}\text { Dalian Institute for } \\
\text { Chemical Physics }\end{array}$ & DMTO $^{\mathrm{c}}$ & $\begin{array}{l}\text { A plant based on SAPO-34 started in Baotou, China } \\
(295 \mathrm{kt} / \mathrm{y})[41]\end{array}$ \\
\hline 2010 & Lurgi & MTP & $\begin{array}{l}\text { First plant started in China }(500 \mathrm{kt} / \mathrm{y} \text { propene and } \\
185 \mathrm{kt} / \mathrm{y} \text { gasoline) [41] }\end{array}$ \\
\hline 2011 & $\begin{array}{l}\text { UOP/INEOS and } \\
\text { Total OCP }\end{array}$ & МТO & $\begin{array}{l}\text { Construction of a plant in Nanjing, China announced } \\
(295 \mathrm{kt} / \mathrm{y}) \text { [41] }\end{array}$ \\
\hline 2012 & ExxonMobil & MTG & $\begin{array}{l}\text { Announced a licensing agreement with Sundrop } \\
\text { Fuels Inc. }(3500 \text { b/d) [79] }\end{array}$ \\
\hline 2012 & UOP & MTO & $\begin{array}{l}\text { Announced a licensing agreement with China's Jiutai } \\
\text { Energy (Zhungeer) Co. Ltd. }(600 \mathrm{kt} / \mathrm{y} \text { ethene and }\end{array}$ \\
\hline
\end{tabular}




\begin{tabular}{|l|l|l|l|l|}
2012 & UOP & MTO & $\begin{array}{l}\text { propene) [80] } \\
\text { Announced a licensing agreement with Shandong } \\
\text { Yangmei Hengtong Chemicals (295 kt/y ethene and } \\
\text { propene).[81] }\end{array}$ \\
UOP & MTO & $\begin{array}{l}\text { Announced a licensing agreement with Jiangsu } \\
\text { Sailboat Petrochemical Co. Ltd.(833 kt/y. Expected } \\
\text { to be the world's largest single MTO plant).[82] }\end{array}$ \\
\hline
\end{tabular}

a: Olefin cracking process, b: Topsøe integrated gasoline synthesis process, c: Dalian methanol process

\subsection{MTH reaction mechanisms}

Within the pores of the zeolites a complex inorganic-organic interplay takes place in which even after the close down of the MTG factory has kept its academic interest. Despite all these years of research there are still unresolved questions regarding the reactions taking place. The formation of $\mathrm{C}-\mathrm{C}$ bonds from methanol is not clearly known. The same is the case for the observed induction period where no methanol is converted.[83] The initial mechanistic work dealt with direct coupling of $\mathrm{C}_{1}$ units from methanol or dimethyl ether (DME). However the energy barrier devoted to the direct coupling is high and the mechanism lacks experimental evidence.[41] Later on the induction period was observed to increase when very purified reactants were, used implicating that the direct $\mathrm{C}_{1}$ coupling was of minor importance compared to impurities for reaction initiation.[84] Today it is generally agreed that the MTH reaction occurs through an indirect reaction mechanism in which hydrocarbons build up inside the zeolite pore system.[41] These reaction centers may be of both aromatic [85-90] and aliphatic (alkene) [91, 92] character, or both [85] and is essential for product formation. Several mechanisms have since the discovery been proposed. In the following an overview of the MTH mechanisms is given.

\section{Alkene mechanism}

In 1982 Dessau et al. [92] presented a mechanism based on repeated alkene methylation and cracking as shown in Figure 26. Lighter alkenes react to higher alkenes by repeated methylation. As the chain grows to e.g. 6 or $7 \mathrm{C}$ atoms cracking may take place. Here it is 
assumed that the initial alkenes were formed by direct $\mathrm{C}-\mathrm{C}$ formation from $\mathrm{C}_{1}$ units, but when alkenes first were formed the reaction leading to their formation is of minor importance compared to methylation and interconversion reactions.

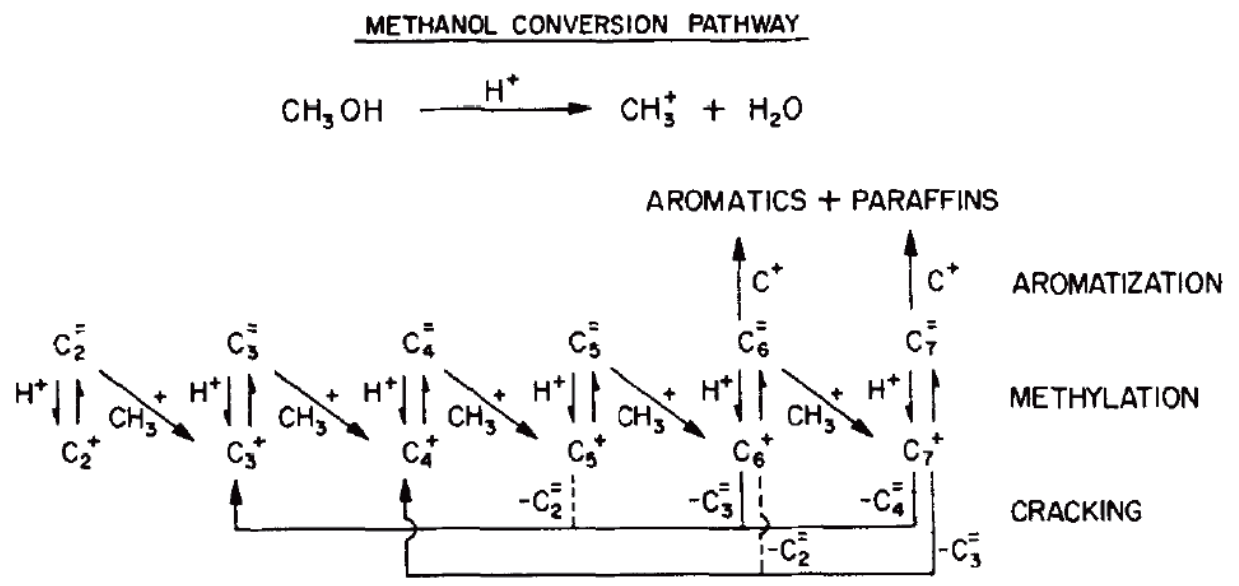

Figure 26: The alkene methylation/cracking mechanism proposed by Dessau et al. [92]

Ethene was expected to be formed from secondary re-equilibration of primary alkenes and not as primary products obtained from methanol.[91] Thus the first alkenes formed are important for the initial reaction period and of only minor importance for the total product amount formed. They speculated that impurities in the methanol, the zeolite or carrier gas could cause initiation of the reaction. In the proposed mechanism aromatics were not presented as reaction intermediates, only as end products involving hydrogen transfer reactions. Later proposed mechanisms changed the picture about the role of aromatics in the MTH reaction.

\section{Aromatic based (hydrocarbon pool) mechanism}

In 1983 Mole et al. [93, 94] investigated the influence of co-reacting p-xylene and toluene with methanol over ZSM-5 and found improved reaction rates for the MTH reaction. They called the effect co-catalysis and the observation was explained by the alkylation of the methyl group attached to aromatic rings in which in subsequent steps alkenes could split off. The importance of cyclic compounds was also supported by 
Langner one year before. [95] In the 1990`s Dahl and Kolboe investigated the MTH reaction over SAPO-34, the zeotype analogue of SSZ-13, by co-reacting alkene precursors (ethanol, propanol) with isotopic labeled $\left({ }^{13} \mathrm{C}\right)$ methanol.[96, 97] When they analyzed the effluent, alkenes were inert and most of the products were exclusively formed from methanol.[96-98] These findings led to the proposal of the "Hydrocarbon pool" mechanism which involves the build up of carbon species inside the pores of the zeolite or zeotype material which act as reaction centers for the MTH reaction as shown in Figure 27.

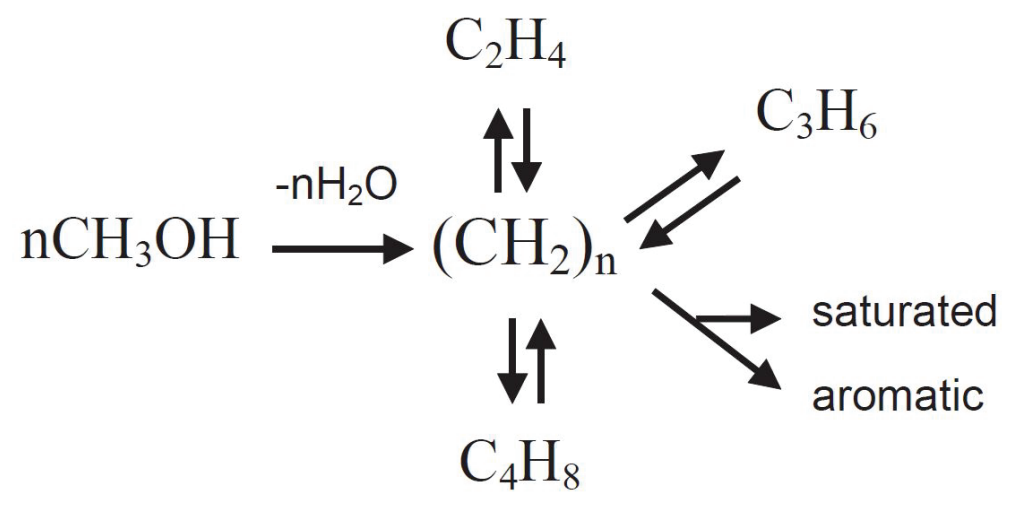

Figure 27: The Hydrocarbon pool mechanism proposed by Dahl and Kolboe. [9698]

Dahl and Kolboe did not give a specific structure of the species involved the hydrocarbon pool. Instead a general formula $\left(\mathrm{CH}_{2}\right)_{\mathrm{n}}$ was presented as the reaction centers which was said to have similarities to ordinary coke and could easily contain species with less hydrogen than given in the formula. Later studies, e.g. isotopic labeling, has shown that methylated benzenes likely are the main reaction centers involved in MTH.[85-90] This is in contrast to Dessau's alkene methylation/cracking mechanism where aromatic species were presented exclusively as end products.

The reaction centers involved may differ dependent on the choice of topology. Both for SAPO-34 and Beta, which have large pores inside the structure, the higher polymethylbenzenes e.g. penta- and hexamethylbenzenes were found to be more active than lower methylated benzenes e.g. toluene and trimethylbenzenes.[90, 99] In the narrower pores of ZSM-5 the lower methyl benzenes were the most active reaction 
centers. $[90,95,99]$ A link between alkene formation and the type of reaction centers has been identified. In Beta the large space available allowed propene and also butenes to split off from the higher (poly-)methylated species [90] while in ZSM-5 ethene and to some extent propene were shown to split off from the reaction centers.[99]

In the hydrocarbon pool mechanism two distinct paths forming alkenes has been presented. One pathway is called the side chain route, the other the paring route and is shown in Figure 28.

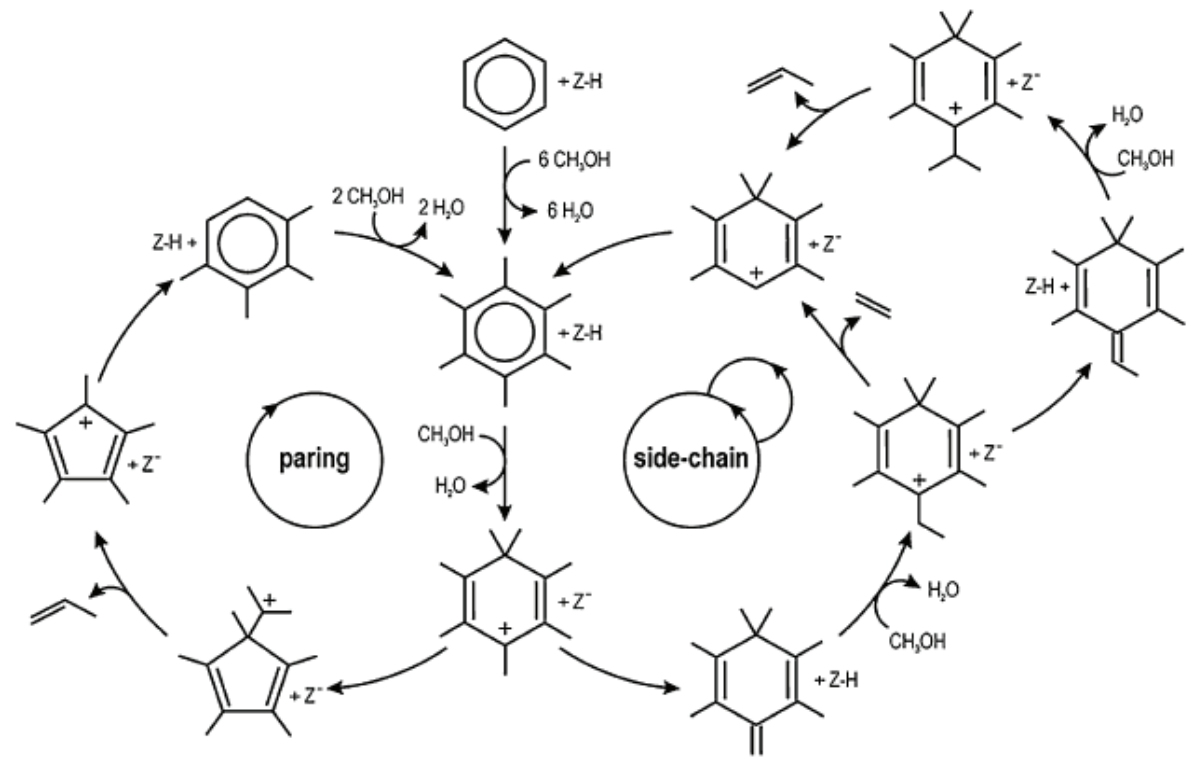

Figure 28: The side chain and the paring route resulting in alkene formation.

Adapted from [100].

The side chain route was first mentioned by Mole et. al $[93,94]$ and later refined by Haw et. al $[101,102]$. In this route heptamethylbenzenium ions deprotonate to 1, 2, 3, 3, 4, 5hexamethyl-6-methylene-cyclohexadiene (HMMC). To the exocyclic double bond a methyl group attaches giving an ethyl group. This can either further methylate to isopropyl and split off as propene or split directly off as ethene. The lower methylbenzenium ions analogues to the higher heptamethylbenzenium ions may also occur as e.g. shown for ZSM-5.[103]

In the paring reaction methyl groups are imagined to be shaved off the 
methylbenzene as alkenes.[41] It was a similar mechanism Sullivan et al.[104] suggested as an explanation to the product formation when hexamethylbenzene was reacted over a bifunctional nickel sulphide on silica-alumina catalyst or over the pure silica-alumina support. The route involves ring contraction from 6-ring to 5-ring and back to a 6-ring. During the ring contraction and expansion the alkyl chain on the ring extends and a carbon atom in the ring and the alkyl group interchange. While the side chain mechanism has been supported experimentally in ZSM-5 [93, 94, 105] the paring route has shown to be favored in Beta [106] and SAPO-5 [107] having larger channels and cages.

\section{The dual cycle mechanism}

The majority of mechanistic investigations of the MTH reaction were until 2006 focused to the spacious zeolite Beta and zeotype SAPO-34 catalysts with higher methylated benzenes as reaction intermediates.[41] Thus much focus was directed towards aromatic compounds as hydrocarbon pool species responsible for product formation. A detailed mechanistic investigation over ZSM-5 was carried out in $2006[85,89]$ In ${ }^{13} \mathrm{C} /{ }^{12} \mathrm{C}$ methanol switching experiments combined with dissolution of the zeolite with HF and analysis of the extracted phase with mass spectrometry, the higher methylbenzenes were found to be almost inert (much slower ${ }^{13} \mathrm{C}$ incorporation as the number of methyl groups attached to the ring increased). This was in contrast to what observed for SAPO-34 and Beta. Additionally the authors showed that ethene and lower methylbenzenes are mechanistically linked through an aromatic based hydrocarbon pool cycle, but separated from the formation of higher alkenes. Aromatic based reaction centers (xylenes and or trimethylbenzenes) were concluded to be responsible for the ethene formation. On the other side the rate of ${ }^{13} \mathrm{C}$ incorporation in $\mathrm{C}_{3}+$ alkenes was much higher than compared to ethene and the most reactive methylbenzenes and showed that propene and higher alkenes mainly forms via repeated methylation and cracking reactions, much the same as the mechanism already presented by Dessau and described above, except that ethene this time was not included. The investigation led to the proposal of the dual cycle concept where two reaction mechanisms work in parallel - the alkene cycle and the aromatic cycle, as shown in Figure 29. 


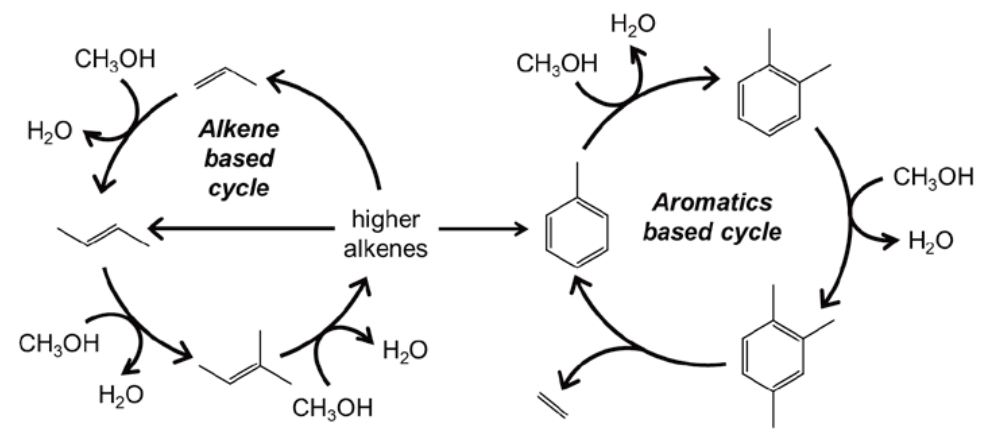

Figure 29: The dual cycle. Adapted from [85].

The dual cycle represents a refinement of the hydrocarbon pool mechanism which was originally based on aromatic reaction centers to also involve species of aliphatic character. Based on these findings the pore space available may have severe influence on the products formed over zeolites or zeotype catalysts. 


\section{Experimental}

\subsection{Synthesis procedures}

\section{Synthesis of TNU-9}

\section{Procedure with premade SDA}

The preparation of TNU-9 was based on the synthesis procedure reported by Hong et al.[108] where 1,4-bis(N-methylpyrrolidinium)-butane (1,4-MPB) was used as a structure directing agent (SDA). 1,4-MPB was made by refluxing 1-methylpyrrolidine (1-MP) and 1,4 dibromobutane $(1,4 \mathrm{DBB})$ with relative molar ratios of $2.5: 1$ in acetone over night (15.4g 1-MP(Fluka, $\geq 98 \%)+75 \mathrm{ml}$ acetone $+16.2 \mathrm{~g}$ 1,4-DBB(Aldrich, 99\%)) under mechanical stirring (Excess of 1-MP facilitates reaction on both ends of the dibromide chain). The product was washed several times with acetone, dried and stored in a desiccator prior to use. The main electron flow is given in Figure 30.

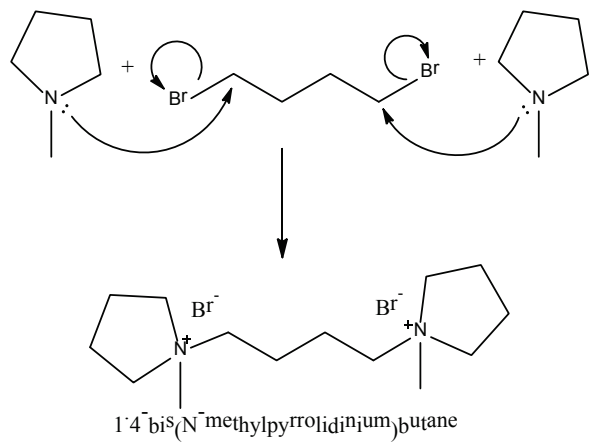

Figure 30: Main electron movement in the SDA synthesis. The high electronegativity of $\mathrm{Br}$ polarises electrons away from the nearest $\mathrm{C}$ atom. The electron pair on the $N$ atom will move closer to the most positive $\mathrm{C}$. Br remains as a charge balancing ion while trimethylamine is connected to the chain.

Pre-made 1,4-MPB, amorphous silica (Cabosil, Riedel de Häen), $\mathrm{Al}\left(\mathrm{NO}_{3}\right)_{3}$. $9 \mathrm{H}_{2} \mathrm{O}$ (Fluka, 98\%) and $\mathrm{NaOH}$ (Merck, $>99 \%$ ) were used as reagents in in the synthesis mixture with relative molar ratios as given in Table 5. 
Table 5: Synthesis conditions in relative molar ratios

\begin{tabular}{lcccccc}
\hline $\mathrm{Na}_{2} \mathbf{O}$ & $\mathbf{A l}_{2} \mathbf{O}_{3}$ & $\mathrm{SiO}_{2}$ & $\mathbf{R}$ & $\mathbf{H}_{2} \mathbf{O}$ & Time & Temperature \\
\hline 10.95 & 0.58 & 30 & 4.5 & 1200 & 14 days & $160{ }^{\circ} \mathrm{C}$ \\
\hline $\mathrm{R}=1.4-\mathrm{MPB}$ & & & & & & \\
\hline
\end{tabular}

The following procedure led to TNU-9 (often multiplied 4 times and divided in separate liners of 45 or $52 \mathrm{ml}$ size.):

1) $\left[0.49 \mathrm{~g} \mathrm{Al}\left(\mathrm{NO}_{3}\right)_{3} \cdot 9 \mathrm{H}_{2} \mathrm{O}+23.47 \mathrm{~g} \mathrm{H}_{2} \mathrm{O}\right]$ Dissolved under mechanical stirring

2) $[(1)+2.00 \mathrm{~g} 50-\mathrm{wt} \% \mathrm{NaOH}$ in water $]$ Added dropwise while stirring to homogeneous solution.

3) $[(2)+1.98 \mathrm{~g}$ 1.4-MPB] Added and stirred.

4) [(1-3) added to 2.06g Cab-o-sil (or Aerosil 200)] Manually stirred for 5 minutes and then mixed mechanical and manual stirring for 30 minutes. It is easier to stir magnetically after some minutes of manual stirring.

5) (4) divided into $52 \mathrm{ml}$ autoclaves and heated to $160{ }^{\circ} \mathrm{C}$ and crystallized under vertical rotation $(37 \mathrm{rpm})$ for 14 days.

6) Autoclaves were quenched, centrifuged and washed with water until $\mathrm{pH} \rightarrow 7$ before drying.

7) The samples were calcined in $30: 70$ ratios of $\mathrm{N}_{2}$ and $\mathrm{O}_{2}$ for a total of $8 \mathrm{~h}, 3 \mathrm{~h}$ to reach $550{ }^{\circ} \mathrm{C}$ and then $5 \mathrm{~h}$ at $550{ }^{\circ} \mathrm{C}$. Calcined samples were ion exchanged in $1 \mathrm{M}$ $\mathrm{NH}_{4} \mathrm{NO}_{3}$ for $2 \mathrm{~h}$ three times at $70{ }^{\circ} \mathrm{C}$ and again calcined to obtain the proton form.

\section{Procedure with in situ formation of SDA}

It is possible to mix the two components, 1-MP and 1,4-DBB directly into the synthesis mixture allowing 1,4-MPB to be formed in situ. The relative molar ratios are given in Table 6.

Table 6: Synthesis conditions in relative molar ratios

\begin{tabular}{ccccccc}
\hline $\mathrm{Na}_{2} \mathbf{O}$ & $\mathrm{Al}_{2} \mathbf{O}_{3}$ & $\mathrm{SiO}_{2}$ & $\mathbf{R}$ & $\mathbf{H}_{2} \mathbf{O}$ & Time & Temperature \\
\hline 10.95 & 0.58 & 30 & $(13.5+4.5)$ & 1200 & 14 days & $160{ }^{\circ} \mathrm{C}$ \\
& & & & & & \\
\hline
\end{tabular}

$\mathrm{R}=13.5$ 1-MP + 4.5 1,4-DBB 
The following procedure led to TNU-9:

1) $\left[1.29 \mathrm{~g} \mathrm{1-MP}+23.13 \mathrm{~g} \mathrm{H}_{2} \mathrm{O}+1.09 \mathrm{~g} 1.4-\mathrm{DBB}\right]$ Added and stirring with magnets.

2) $\left[(1)+0.49 \mathrm{~g} \mathrm{Al}\left(\mathrm{NO}_{3}\right)_{3} \cdot 9 \mathrm{H}_{2} \mathrm{O}\right]$ Added while stirring.

3) $[(1-2)+1.97 \mathrm{~g} 50-\mathrm{wt} \% \mathrm{NaOH}$ in water $]$ Added dropwise while stirring to homogeneous solution.

4) [(1-3) added to 2.03g Cabosil (or Aerosil 200)] Manually stirred for 5 minutes and then mixed mechanical and manual stirring for 30 minutes. It was easier to stir magnetically after some minutes of manual stirring.

5) (4) divided into $52 \mathrm{ml}$ autoclaves and heated to $160{ }^{\circ} \mathrm{C}$ and crystallized under vertical rotation $(37 \mathrm{rpm})$ for 14 days.

6) Autoclaves were quenched, centrifuged and washed with water until $\mathrm{pH} \rightarrow$ before drying.

7) The samples were calcined in $30: 70$ ratios of $\mathrm{N}_{2}$ and $\mathrm{O}_{2}$ for a total of $8 \mathrm{~h}, 3 \mathrm{~h}$ to reach $550{ }^{\circ} \mathrm{C}$ and then $5 \mathrm{~h}$ at $550{ }^{\circ} \mathrm{C}$. Calcined samples were ion exchanged in $1 \mathrm{M}$ $\mathrm{NH}_{4} \mathrm{NO}_{3}$ for $2 \mathrm{~h}$ three times at $70{ }^{\circ} \mathrm{C}$ and again calcined to obtain the proton form.

\section{Comments to the procedures of TNU-9}

TNU-9 has shown to crystallize in a very narrow range of gel composition. It has been extensively investigated and several attempts have been made to expand the $\mathrm{Si} / \mathrm{Al}$ ratio (change of template to hexamethonium bromide, ion exchange of 1,4-MPB template to $\mathrm{OH}$-form and replacing $\mathrm{NaOH}$ with $\mathrm{HF}$, Ge incorporation, time studies, temperature and static vs. tumbling conditions etc.). These trials did not lead to the expected phase. The recipe presented here is in the very narrow range in which the structure can be crystallized. Tumbling under crystallization leads to large reduction in synthesis time. The in situ formation of SDA avoids the need for premaking 1,4-MPB which is hygroscopic.

\section{Synthesis of IM-5}

\section{Procedure with premade SDA}

IM-5 was prepared as stated in [22] by using 1,5-bis(N-metylpyrrolidinium)pentane (1,5MPP) as a SDA. The SDA was prepared by refluxing (under stirring) 1,5-dibromopentane 
(1,5-DBP) with 1-MP with relative molar ratios $2.5: 1$ in acetone over night (13.9g 1-MP $($ Fluka,$\geq 98 \%)+75 \mathrm{ml}$ Acetone $+15.0 \mathrm{~g}$ 1.5-DBP (Aldrich, 97\%)). The product was recovered by filtration and washed several times with acetone before drying and storing in a desiccator prior to use.

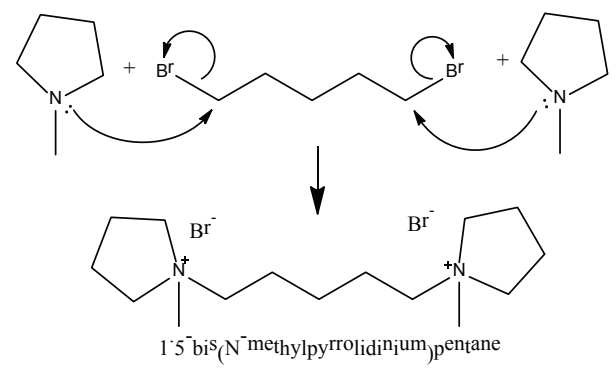

Figure 31: Synthesis of 1.5-bis(N-methylpyrrolidinium)pentane.

Pre-made 1,5-MPP, Amorphous silica (Cab-osil, Riedel de Häen), $\mathrm{Al}\left(\mathrm{NO}_{3}\right)_{3} \cdot 9 \mathrm{H}_{2} \mathrm{O}$ (Fluka, $>98 \%$ ) and $\mathrm{NaOH}$ (Merck, $>99 \%$ ) were used as reagents in the synthesis mixture with relative molar ratios as given in Table 7.

Table 7: Synthesis conditions in relative molar ratios

\begin{tabular}{|c|c|c|c|c|c|c|}
\hline $\mathrm{Na}_{2} \mathrm{O}$ & $\mathrm{Al}_{2} \mathbf{O}_{3}$ & $\mathrm{SiO}_{2}$ & $\mathbf{R}$ & $\mathrm{H}_{2} \mathrm{O}$ & Time & Temperature \\
\hline 10.95 & $(0.5-) 0.6$ & 30 & 3.00 & 1200 & 14 days & $160^{\circ} \mathrm{C}$ \\
\hline
\end{tabular}

IM-5 was typically prepared by:

1) $\left[1.75 \mathrm{~g} \mathrm{Al}\left(\mathrm{NO}_{3}\right)_{3} \cdot 9 \mathrm{H}_{2} \mathrm{O}+95.90 \mathrm{~g} \mathrm{H}_{2} \mathrm{O}\right]$ Added and stirred mechanically

2) $[(1)+8.16 \mathrm{~g} 50 \mathrm{wt}-\%$ aqueous solution of $\mathrm{NaOH}]$ Added while stirring

3) $[(2)+5.79 \mathrm{~g}$ 1,5-MPP] Added while stirring

4) $[8.40 \mathrm{~g}$ Cabosil $+(3)]$ Added and manually stirred for 5 minutes. Then mixed manually and mechanically stirred for 30 minutes.

5) (4) divided into four $45 \mathrm{ml}$ autoclaves and heated to $160{ }^{\circ} \mathrm{C}$ for $14-18$ days under vertical rotation $(50 \mathrm{rpm})$.

6) Crystallized product was quenched and repeatedly washed with water to $\mathrm{pH}$ was around 7 before drying. 
7) The samples were calcined at $550{ }^{\circ} \mathrm{C}$ for $8 \mathrm{~h}, 3 \mathrm{~h}$ to reach $550{ }^{\circ} \mathrm{C}$ and then held at this temperature for $5 \mathrm{~h}$, in a flow of $30: 70 \mathrm{~N}_{2}$ and $\mathrm{O}_{2}$. Calcined samples were ion exchanged in $1 \mathrm{M} \mathrm{NH}_{4} \mathrm{NO}_{3}$ solution three times for $2 \mathrm{~h}$ at $70{ }^{\circ} \mathrm{C}$. Dried samples were again calcined to obtain the proton form.

\section{Comments to the procedure for IM-5}

Similar to TNU-9, IM-5 crystallized only in a narrow range of synthesis compositions. In situ formation of template worked, but led to lower crystallinity than obtained for TNU-9. Despite many trials similar to TNU-9 the Si/Al ratio was difficult to expand with these flexible templates. Again, tumbling during the crystallization reduced the synthesis time dramatically.

\section{Synthesis of ITQ-13}

\section{Ion-exchange of SDA}

ITQ-13 is typically synthesized by the fluoride route in highly concentrated synthesis mixtures using hexamethonium hydroxide as a structure directing agent (SDA).[19, 40] The SDA in hydroxide form was pre-made by dissolving $54 \mathrm{~g}$ hexamethonium bromide (J\&K Scientific, $>98 \%$ ) in water and ion exchanged with $300 \mathrm{~g}$ amberlite IRN-78 resin in a column. The template solution was then rotaevaporated to a solution which typically ended up around $20-25 \mathrm{wt}-\% \mathrm{HM}(\mathrm{OH})_{2}$ in water. The wt- $\%$ was determined by titration with $0.1 \mathrm{~N} \mathrm{HCl}$ using BTB as an indicator.

\section{Synthesis procedure}

Ion exchanged $\mathrm{HM}(\mathrm{OH})_{2}$, Al-isopropoxide, TEOS, HF (40 wt- $\%$ in water) were used as reagents in the synthesis which had final relative molar ratios as given in Table 8.

Table 8: Synthesis conditions in relative molar ratios

\begin{tabular}{cccccccc}
\hline $\mathbf{S i O}_{2}$ & $\mathrm{Al}_{2} \mathbf{O}_{3}$ & $\mathrm{GeO}_{2}$ & $\mathrm{HF}$ & $\mathbf{H M}(\mathbf{O H})_{2}$ & $\mathbf{H}_{2} \mathbf{O}$ & Time & Temperature \\
\hline 0.910 & 0.010 & 0.090 & 0.56 & 0.250 & 5 & $(10-) 15$ days & $160{ }^{\circ} \mathrm{C}$ \\
\hline
\end{tabular}


The synthesis was performed in plastic beakers (because of HF) with the following procedure:

(1) $\left[0.46 \mathrm{~g} \mathrm{GeO}_{2}+11.75 \mathrm{~g} 24.5 \mathrm{wt}-\% \mathrm{HM}(\mathrm{OH})_{2}\right.$ aqueous solution]*

(2) $[(1)+0.20 \mathrm{~g} \mathrm{Al}$ isopropoxide] Dissolved in a small amount of isopropoxide and added while stirring.

(3) $[(1-2)+9.26 \mathrm{~g}$ TEOS $]$ Added dropwise under stirring

(4) The plastic beaker with the synthesis mixture was weighed and put for stirring 1-2 $\mathrm{h}$ at ambient temperature and then heated to around $80^{\circ} \mathrm{C}$ (while stirring) until all the ethanol $(8.18 \mathrm{~g})$, the small amount of isopropoxide and the excess water $(2.88 \mathrm{~g})$ had evaporated.

(5) $[(1-4)+1.36 \mathrm{~g}$ HF (40 wt-\% in aqueous solution) $]$ added dropwise and stirred manually (get very dry after adding of HF)

(6) The final mixture was added to $52 \mathrm{ml}$ teflon liners in a stainless steel autoclave and kept in a tumbling oven (vertical rotation) at $37 \mathrm{rpm}$ for 14 days at $160^{\circ} \mathrm{C}$

(7) Then the autoclave was quenched and the product was washed several times with distilled water to $\mathrm{pH}$ was close to neutral.

(8) After drying the product was calcined in $50: 50 \mathrm{~N}_{2}$ and $\mathrm{O}_{2}$ for $8 \mathrm{~h}$. Three hours from room temperature to $550^{\circ} \mathrm{C}$ and at $550^{\circ} \mathrm{C}$ for 5 hours.

*By adding seeds the nucleation is facilitated. In the synthesis pure Si-ITQ-13 can be made prior to the synthesis by the same procedure excluding Ge and Al. The synthesis mixture for making seeds had a final molar composition of $1 \mathrm{SiO}_{2}: 0.5$ $\mathrm{HF}: 0.25 \mathrm{HM}(\mathrm{OH})_{2}: 5.00 \mathrm{H}_{2} \mathrm{O}$. Typically (up to) $0.11 \mathrm{~g}$ seeds were added to the recipe for the $\mathrm{Al}$ and $\mathrm{Ge}$ containing ITQ-13 described above to improve nucleation rates and reduce the risk of failed reproduction.

\section{Comments to the procedure for ITQ-13}

It is necessary to take into account that every mole of TEOS consumes two moles of water to obtain the final molar composition. Thus the consumed water has to be added on top of the final molar composition. This structure was difficult to obtain the first times, but as long as seed is used it is rather easy to reproduce as this helps the nucleation 
leading to further crystal growth. When the $\mathrm{Si} / \mathrm{Al}$ ratio is increased with low amount of Ge present a typical morphology change from rod/rise/needle shaped to plate like particles was observed. Al is much easier to introduce when Ge is present. Alternatively it can be synthesized in as B-ITQ-13 and then B can be substituted by Al in post synthesis treatment, not attempted here.[40]

\section{Synthesis of EU-1}

EU-1 was synthesized by rather similar procedures as reported in [20, 109] by using commercially available $\mathrm{HMBr}_{2}(\mathrm{~J} \& \mathrm{~K}$ Scientific, $>98 \%)$ as a template. $\mathrm{Al}\left(\mathrm{NO}_{3}\right)_{3} \cdot 9 \mathrm{H}_{2} \mathrm{O}$ (Fluka, >98\%), $\mathrm{NaOH}$ (Merck, >99\%) diluted to 50 wt-\% in water and Ludox AS-30 (Aldrich, $30 \mathrm{wt}-\%$ ) were used as reagents in a synthesis mixture with the following relative molar ratios:

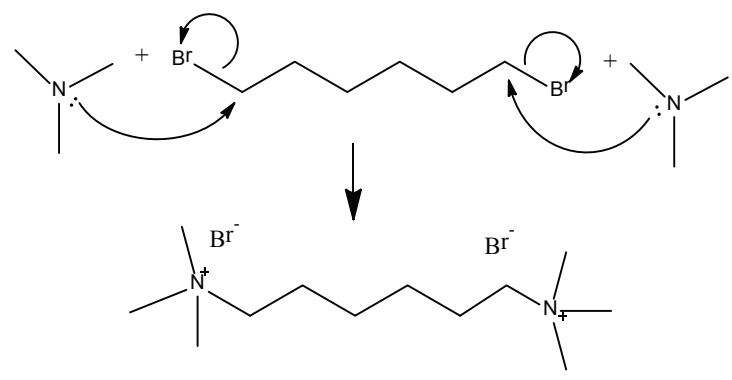

$N^{1,} N^{1,} N^{1,} N^{6,} N^{6,} N^{6}{ }^{-}$examethylhexane ${ }^{-}{ }_{1}, 6^{-} \mathrm{di}^{\mathrm{am}} \mathrm{i}_{\mathrm{i}} \mathrm{n}_{\mathrm{i}} \mathrm{um}_{\mathrm{b}} \mathrm{rom}_{\mathrm{ide}}$

Figure 32: Synthesis of $\mathrm{HMBr}_{2}$.

Table 9: Synthesis conditions in relative molar ratios

\begin{tabular}{ccccccc}
\hline $\mathrm{Na}_{2} \mathbf{O}$ & $\mathrm{Al}_{2} \mathbf{O}_{3}$ & $\mathbf{S i O}_{2}$ & $\mathbf{H M B r}_{2}$ & $\mathbf{H}_{2} \mathbf{O}$ & Time & Temperature \\
\hline 9 & 0.5 & 30 & 4.5 & 1335 & $(10-) 14$ days & $160{ }^{\circ} \mathrm{C}$
\end{tabular}

A typical synthesis of EU-1 is given here:

(1) $\left[80.08 \mathrm{~g} \mathrm{H}_{2} \mathrm{O}+6.08 \mathrm{~g} 50\right.$ wt- $\left.\% \mathrm{NaOH}\right]$ Mixed and mechanically stirred

(2) $\left[(1)+1.58 \mathrm{~g} \mathrm{Al}\left(\mathrm{NO}_{3}\right)_{3} \cdot 9 \mathrm{H}_{2} \mathrm{O}\right]$ Added under stirring

(3) $\left[(1-2)+6.88 \mathrm{~g} \mathrm{HMBr}_{2}\right]$ Added under stirring 
(4) $[(1-3)+25.37 \mathrm{~g}$ Ludox AS-30] Added under mechanical/manual stirring

(5) The gel was transferred to $45 / 52 \mathrm{ml}$ teflon liners in stainless steel autoclaves and crystallized in a tumbling oven $(37 \mathrm{rpm})$ for $(10-) 14$ days at $160^{\circ} \mathrm{C}$.

(6) The autoclaves were quenched and the product was washed several times with distilled water to $\mathrm{pH}$ was close to neutral.

(7) After drying the product was calcined in $50: 50 \mathrm{~N}_{2}$ and $\mathrm{O}_{2}$ for $8 \mathrm{~h}$. Three hours from room temperature to $550^{\circ} \mathrm{C}$ and at $550^{\circ} \mathrm{C}$ for 5 hours, ion exchanged $3 \times 2 \mathrm{~h}$ in $1 \mathrm{M} \mathrm{NH}_{4} \mathrm{NO}_{3}$ and again calcined after drying.

\section{Synthesis of ZSM-48}

ZSM-48 was synthesized with pentamethonium bromide $\left(\mathrm{PMBr}_{2}\right)$ as the SDA.[20] The SDA was premade by mixing $15 \mathrm{~g}$ dibromopentane (DBP, Sigma-Aldrich, 97\%) with 75 $\mathrm{ml}$ ethanol and $31.1 \mathrm{~g} 33 \mathrm{wt} \%$ trimethylamine (TMA, 31-35\%) solution in ethanol, giving a relative molar ratio of $2.5: 1$ of TMA/DBP. The mixture was refluxed over night, filtered and dried at $70^{\circ} \mathrm{C}$ prior to use.

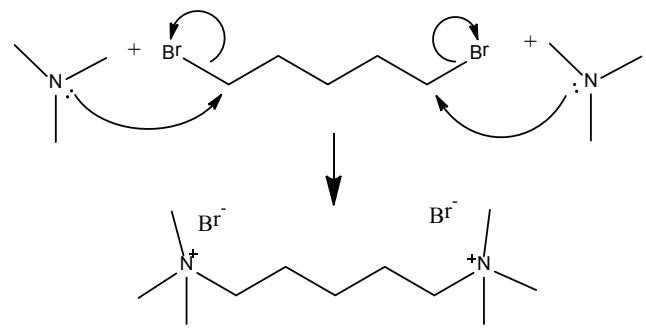

$N^{1,} N^{1,} N^{1,} N^{5,} N^{5,} N^{5^{-}}$hexamethylpentane ${ }^{-}, 5^{-}$diam $_{i} n_{i} u m$ bromide

Figure 33: Synthesis of $\mathrm{PMBr}_{2}$.

In the synthesis $\mathrm{Al}\left(\mathrm{NO}_{3}\right)_{3} \cdot 9 \mathrm{H}_{2} \mathrm{O}$ (Fluka, $>98 \%$ ), $\mathrm{NaOH}$ (Merck, >99\%) diluted to $50 \mathrm{wt}-\%$ in water, $\mathrm{PMBr}_{2}$ and TEOS (Aldrich) were used as reagents in the synthesis mixture with relative molar ratios given in Table 10 .

Table 10: Synthesis conditions in relative molar ratios

\begin{tabular}{ccccccc}
\hline $\mathrm{Na}_{2} \mathbf{O}$ & $\mathrm{Al}_{2} \mathbf{O}_{3}$ & $\mathrm{SiO}_{2}$ & $\mathbf{P M B r}_{2}$ & $\mathbf{H}_{2} \mathbf{O}$ & Time & Temperature \\
\hline 5 & 0.375 & 30 & 4.5 & 1200 & 7 days & $160{ }^{\circ} \mathrm{C}$ \\
& & & & & & \\
\hline
\end{tabular}


A typical synthesis of ZSM-48 is given here:

(1) $\left[0.22 \mathrm{~g} \mathrm{Al}\left(\mathrm{NO}_{3}\right)_{3} \cdot 9 \mathrm{H}_{2} \mathrm{O}+17.93 \mathrm{~g} \mathrm{H}_{2} \mathrm{O}\right]$ Mixed and mechanically stirred

(2) $[(1)+0.64 \mathrm{~g} 50 \mathrm{wt}-\% \mathrm{NaOH}]$ Added and stirred

(3) $\left[(1-2)+1.24 \mathrm{~g} \mathrm{PMBr}_{2}\right]$ Added under stirring

(4) $[(1-3)+4.96 \mathrm{~g}$ TEOS $]$ Added dropwise under mechanical stirring

(5) The beaker was weighed (including the content) and left under stirring for $1 \mathrm{~h}$ at room temperature before the beaker was heated to ensure that all methanol had evaporated.

(6) The gel was transferred to a $45 \mathrm{ml}$ teflon liner in stainless steel autoclaves and crystallized in a tumbling oven $(37 \mathrm{rpm})$ for 7 days at $160^{\circ} \mathrm{C}$.

(7) The autoclaves were quenched and the product was washed several times with distilled water to $\mathrm{pH}$ was close to neutral.

(8) After drying the product was calcined in $50: 50 \mathrm{~N}_{2}$ and $\mathrm{O}_{2}$ for $8 \mathrm{~h}$. Three hours from room temperature to $550^{\circ} \mathrm{C}$ and at $550^{\circ} \mathrm{C}$ for 5 hours, ion exchanged $3 \times 2 \mathrm{~h}$ in $1 \mathrm{M} \mathrm{NH}_{4} \mathrm{NO}_{3}$ and again calcined after drying to obtain the proton form.

\section{Synthesis of ZSM-22}

A commercial sample was supplied from Zeolyst International. In house made ZSM-22 (H) was based on [110-112] with minor modifications. In the synthesis $\mathrm{Al}_{2}\left(\mathrm{SO}_{4}\right)_{3}$. $18 \mathrm{H}_{2} \mathrm{O}$ (J. T. Baker), $\mathrm{KOH}$ (Merck) diluted to $50 \mathrm{wt}-\%$ in water, diaminooctane (DAO) (Fluka) and Ludox AS-30 (Aldrich) were used as reagents in the synthesis mixture with relative molar ratios given in

Table 11: Synthesis conditions in relative molar ratios

\begin{tabular}{cccccccc}
\hline $\mathbf{K}_{2} \mathbf{O}$ & $\mathbf{K}_{2} \mathbf{S O}_{4}$ & $\mathbf{A l}_{2} \mathbf{O}_{3}$ & $\mathbf{S i O}_{2}$ & $\mathrm{DAO}$ & $\mathbf{H}_{2} \mathbf{O}$ & Time & Temperature \\
\hline 8.9 & 3 & 1 & 90 & 27.3 & 3588 & $3-4$ days & $160{ }^{\circ} \mathrm{C}$ \\
\hline
\end{tabular}

A typical synthesis of ZSM-22 is given here:

(1) $\left[2.39 \mathrm{~g} \mathrm{KOH}+10.89 \mathrm{~g} \mathrm{H}_{2} \mathrm{O}\right]$ Dissolved in beaker 1 
(2) $\left[1.06 \mathrm{~g} \mathrm{Al}_{2}(\mathrm{SO})_{4} \cdot 18 \mathrm{H}_{2} \mathrm{O}+10.89 \mathrm{~g} \mathrm{H}_{2} \mathrm{O}\right]$ Dissolved in beaker 2

(3) $\left[6.23 \mathrm{~g} \mathrm{DAO}+43.6 \mathrm{~g} \mathrm{H}_{2} \mathrm{O}\right]$ Dissolved in beaker 3

(4) Beaker 1-3 was mixed and put for mechanical stirring

(5) $\left[28.5 \mathrm{~g}\right.$ Ludox AS-30 $\left.+16.14 \mathrm{~g} \mathrm{H}_{2} \mathrm{O}\right]$ Mixed in beaker 4

(6) $[(1-4)+5]$ added under vigorous stirring.

(7) The gel was transferred to $45 \mathrm{ml}$ teflon liners in stainless steel autoclaves and crystallized in a tumbling oven $(37 \mathrm{rpm})$ for 3-4 days at $160^{\circ} \mathrm{C}$.

(8) The autoclaves were quenched and the product was washed several times with distilled water to $\mathrm{pH}$ was close to neutral.

(9) After drying the product was calcined at $550^{\circ} \mathrm{C}$ for $12 \mathrm{~h}$ a flow of pure oxygen. The calcined samples were ion exchanged $3 \times 2 \mathrm{~h}$ in $1 \mathrm{M} \mathrm{NH} \mathrm{NHO}_{3}$ and again calcined for $2 \mathrm{~h}$ at $550^{\circ} \mathrm{C}$ in static air after drying to obtain the proton form.

\section{Comments to the procedure for ZSM-22}

Proper calcination particularly important due to narrow pores and needle shaped crystals with channels oriented along the needle. During the calcination pure oxygen should be used. XRD results from the recipe described may give small amounts of a dense phase called cristobalite. A more detailed description of different synthesis procedures and results of ZSM-22 than herein presented can be found in [113].

The commercial ZSM-22 was supplied from Zeolyst International.

\section{Synthesis of ZSM-23}

The commercial sample was supplied from Zeolyst International.

In house synthesized ZSM-23 was based on [20] using heptamethonium bromide as a SDA. The SDA was premade by refluxing $15 \mathrm{~g}$ dibromoheptane in $75 \mathrm{ml}$ ethanol with 27.71 g 33 wt- $\%$ trimethylamine (TMA, 31-35\% solution in ethanol), giving a relative molar ratio of $2.5: 1$ of TMA/DBHep. The mixture was refluxed over night, filtered (and eventually washed with acetone) and dried at $70^{\circ} \mathrm{C}$ prior to use. (Comments to the recipe: (1) the amount of ethanol can be reduced, (2) herein the crystals precipitated after cooling). 


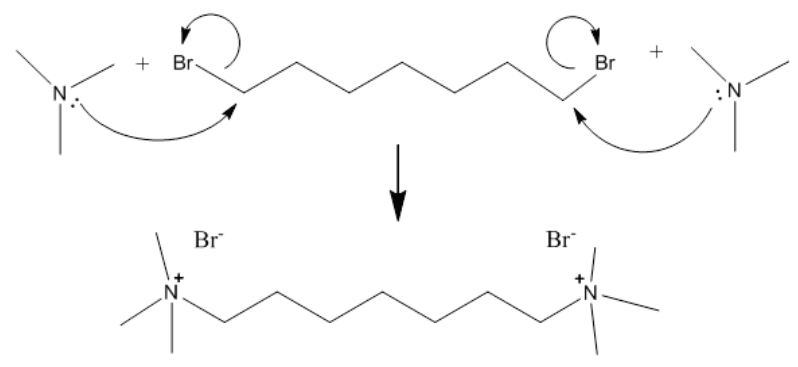

$N 1, \mathrm{~N} 1, \mathrm{~N} 1, \mathrm{~N} 7, \mathrm{~N} 7, \mathrm{~N} 7$-hexamethylheptane-1,7-diaminium bromide

Figure 34: Synthesis of hepMBr 2

In the synthesis $\mathrm{Al}\left(\mathrm{NO}_{3}\right)_{3} \cdot 9 \mathrm{H}_{2} \mathrm{O}$ (Fluka, $>98 \%$ ), $\mathrm{NaOH}$ (Merck, $>99 \%$ ) diluted to 50 wt- $\%$ in water, $\mathrm{HepMBr}_{2}$ and Cabosil (Riedel de Häen) were used as reagents in the synthesis mixture with relative molar ratios given in

Table 12: Synthesis conditions in relative molar ratios

\begin{tabular}{ccccccc}
\hline $\mathrm{Na}_{2} \mathrm{O}$ & $\mathrm{Al}_{2} \mathbf{O}_{3}$ & $\mathrm{SiO}_{2}$ & $\mathrm{HepMBr}_{2}$ & $\mathbf{H}_{2} \mathbf{O}$ & Time & Temperature \\
\hline 5 & 0.5 & 30 & 4.5 & 1200 & $7(-14)$ days ${ }^{\mathrm{a}}$ & $160{ }^{\circ} \mathrm{C}$ \\
& & & & & & \\
\hline${ }^{\mathrm{a}}$ Tumbling & & & & & &
\end{tabular}

A typical synthesis procedure is given here:

1) $\left[1.53 \mathrm{~g} 50 \mathrm{wt}-\% \mathrm{NaOH}+41.06 \mathrm{~g} \mathrm{H}_{2} \mathrm{O}\right]$ Added and stirred

2) $\left[(1)+0.73 \mathrm{~g} \mathrm{Al}\left(\mathrm{NO}_{3}\right)_{3} \cdot 9 \mathrm{H}_{2} \mathrm{O}\right]$ Added while stirring, and stirred to dissolved

3) $\left[(2)+3.24 \mathrm{~g} \mathrm{HepMBr}_{2}\right]$ Added while stirred, stirred to homogeneous solution

4) $[3.45$ g Cabosil + (3)] Added to Cabosil and stirred manually for $5 \mathrm{~min}$. Then mechanically for some minutes and manually again.

5) Divided on two $45 \mathrm{ml}$ teflon liners and heated in autoclaves at $160^{\circ} \mathrm{C}$ for $7-14$ days under tumbling conditions.

6) The autoclaves were quenched and the product was washed several times with distilled water to $\mathrm{pH}$ was close to neutral.

7) After drying the product was calcined $9 \mathrm{~h}$ in $\mathrm{O}_{2}, 4 \mathrm{~h}$ to reach $550^{\circ} \mathrm{C}$ and then kept at $550^{\circ} \mathrm{C}$ for $5 \mathrm{~h}$. The calcined samples were ion exchanged $3 \times 2 \mathrm{~h}$ in $1 \mathrm{M} \mathrm{NH}_{4} \mathrm{NO}_{3}$ and again calcined after drying to obtain the proton form. 


\section{Comments to the procedure for ZSM-23}

In [20] a Si/Al range between 30-60 can be obtained with $\mathrm{HepMBr}_{2}$ as a SDA when the $\mathrm{Na}_{2} \mathrm{O}$ amount is kept constant as described. In the original article they used 7 days while herein it was increased up to 14 days. Such a long crystallization time should therefore not be necessary.

\section{Synthesis of ZSM-5}

The sample pz-2/100H and pz-2/25H was supplied from ZeoCat.

\section{Synthesis of ZSM-57}

ZSM-57 was synthesized in alkaline media by using $\mathrm{N}, \mathrm{N}, \mathrm{N}, \mathrm{N}, \mathrm{N}^{\prime}, \mathrm{N}^{\prime}$ hexaethylpentanediammonium (Et6-diquat-5 in bromide version) as a structure directing agent (SDA), Figure 35. The SDA was pre-made by refluxing triethylamine (Sigma, $\geq$ $99.5 \%)$ and dibromopentane (2.5: 1 ratio) in acetone under continuous stirring over night. The SDA was recovered by filtration, washed with acetone and dried prior to use.

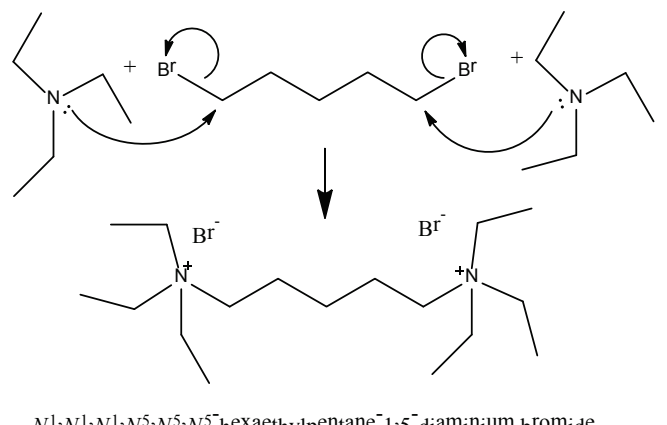

Figure 35: Synthesis of Et6-diquat-5.

Colloidal silica, Ludox AS-30 (30 wt-\% in water, Sigma-Aldrich), $\mathrm{Al}\left(\mathrm{NO}_{3}\right)_{3} \cdot 9 \mathrm{H}_{2} \mathrm{O}(98$ $\%$, Fluka), $\mathrm{NaOH}$ (Merck) were used as reagents in the synthesis mixture with a relative composition as given in Table 13.

Table 13: Synthesis conditions in relative molar ratios

\begin{tabular}{ccccccc}
\hline $\mathbf{N a}_{2} \mathbf{O}$ & $\mathbf{A l}_{2} \mathbf{O}_{3}$ & $\mathbf{S i O}_{2}$ & $\mathbf{E t}_{\mathbf{6}}$-diquat-5 & $\mathbf{H}_{2} \mathbf{O}$ & Time & Temperature \\
\hline$(6.9-) 7.2$ & 0.5 & 30 & 3.0 & 1200 & 14 days & $160{ }^{\circ} \mathrm{C}$
\end{tabular}


A typical synthesis procedure of ZSM-57 is given here:

(1) $\left[1.13 \mathrm{~g} 50 \mathrm{wt}-\% \mathrm{NaOH}+16.34 \mathrm{~g} \mathrm{H}_{2} \mathrm{O}\right]$ Mixed and mechanically stirred

(2) $\left[(1)+0.37 \mathrm{~g} \mathrm{Al}\left(\mathrm{NO}_{3}\right)_{3} \cdot 9 \mathrm{H}_{2} \mathrm{O}\right]$ Added under stirring

(3) $\left[(1-2)+1.27 \mathrm{~g} \mathrm{Et}_{6}\right.$-diquat-5 (Bromide version) $]$ Added under stirring

(4) $[(1-3)+5.89 \mathrm{~g}$ Ludox AS-30] Added under mechanical/manual stirring

(5) The gel was transferred to $45 / 52 \mathrm{ml}$ teflon liners in stainless steel autoclaves and crystallized in a tumbling oven $(24 \mathrm{rpm})$ for 14 days at $160^{\circ} \mathrm{C}$.

(6) The autoclaves were quenched and the product was washed several times with distilled water to $\mathrm{pH}$ was close to neutral.

(7) After drying the product was calcined in $50: 50 \mathrm{~N}_{2}$ and $\mathrm{O}_{2}$ for $8 \mathrm{~h}$. Three hours from room temperature to $550^{\circ} \mathrm{C}$ and at $550^{\circ} \mathrm{C}$ for 5 hours, ion exchanged $3 \times 2 \mathrm{~h}$ in $1 \mathrm{M} \mathrm{NH}_{4} \mathrm{NO}_{3}$ and again calcined after drying.

\subsection{Experimental Methods}

\subsubsection{Characterisation methods}

\section{X-Ray Diffraction (XRD)}

The crystallinity and purity of the material was determined using X-ray diffraction (XRD). A Siemens Bruker D5000 instrument with Bragg-Brentano geometry and $\mathrm{Cu} \mathrm{K} \alpha 1$ radiation $(\lambda=1.5406 \AA)$ was used for the measurements. Dried samples were fine grinded by a mortar. Full sample holders (sometimes glass sample holders) were used for phase identification on all as-synthesized samples. The diffractograms were analyzed with EVA 8.0 (SOCABIM) and the diffractograms were compared with the powder diffraction file (PDF) base revised by Joint Committee on Powder Diffraction Standards in cases where the zeolite was registered. TNU-9, IM-5 and ITQ-13 were not added in the database and in these cases the diffraction patterns were calculated in Materials Studio 5.0 based on the cif-files found in the IZA database. 


\section{Scanning Electron Microscopy (SEM)}

SEM was used to determine crystal size and shape as well as impurities. Element analysis (EDX) was used to determine the chemical composition (e.g. the $\mathrm{Si} / \mathrm{Al}$ ratio) of the crystals. The zeolite powder was dispersed on a carbon tape, eventually with a thin layer of Pt or Au to avoid charging, and analyzed. A FEI Quanta 200 FEG-ESEM was used for the analysis.

\section{Transmission Electron Microscopy (TEM)}

TEM analysis was used to identify the direction of channels relative to crystal orientations in shape-selectivity-morphology relations. The analyses were performed on Philips C200 at Haldor Topsøe in Denmark.

\section{Sorption measurements}

$\mathrm{N}_{2}$ sorption measurements were used to determine the surface area and pore volume of the zeolites. About $55 \mathrm{mg}$ catalyst was weighed and measured in a relative partial pressure range from $0-0.99 \mathrm{P} / \mathrm{P}_{0}$ at liquid $\mathrm{N}_{2}$ temperature. Prior to the measurement the sample was outgassed at $80^{\circ} \mathrm{C}$ for $1 \mathrm{~h}$ and then at $300^{\circ} \mathrm{C}$ for $4 \mathrm{~h}$. A BELSORP mini-II was used in the measurement.

The BJH method was used to evaluate the mesopore distribution. This method is based on the Kelvin equation which relates the lowering of the pressure in which the adsorbate condenses when narrow pores are present to the radius of the pore. The Kelvin equation does not take into account the multilayer thickness $(t)$ on the walls. Thus the Kelvin equation only calculate the Kelvin radius $\left(r_{k}\right)$ as shown in Figure 36 and not the real pore size $r_{p}=r_{k}+t$. 


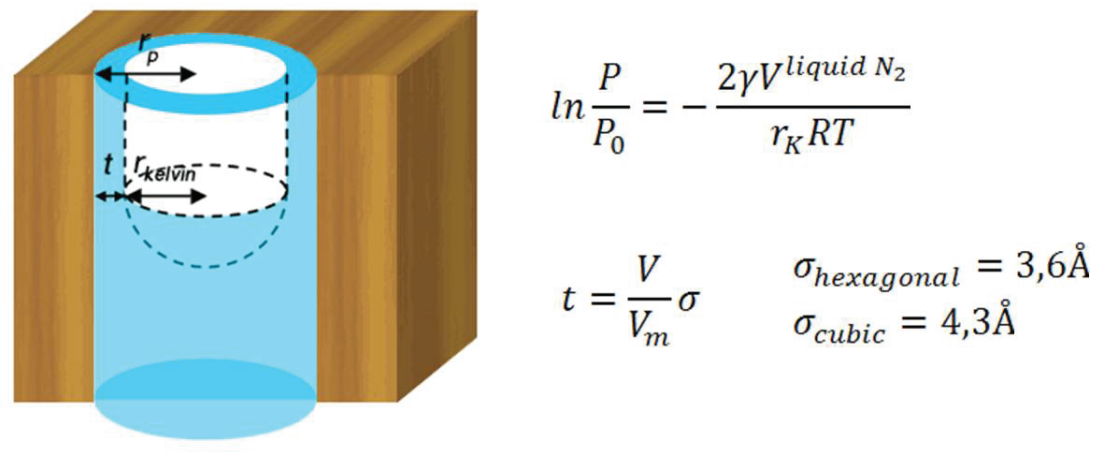

Figure 36: The Kelvin equation is used to calculate the mesopore distribution. However the statistical thickness of adsorbate layer (t) has to be calculated, normally from BET data. The figure is adapted from [114].

The latter multilayer thickness is often calculated from BET data (number of molecules adsorbed at a given pressure divided on the number of molecules adsorbed in one monolayer multiplied by the effective height $(\sigma)$ e.g. assuming hexagonal or cubic arrangement of the adsorbed gas. From this equation and the results from the sorption measurements it is possible to estimate the pore size distribution of the material. The results are presented as a plot where the change in volume per change in radius $\left(\mathrm{dV}_{\mathrm{p}} / \mathrm{dr}_{\mathrm{p}}\right)$ versus the pore radius. The $\mathrm{dV}_{\mathrm{p}} / \mathrm{dr}_{\mathrm{p}}$ represents in other words the slope of the change in volume of adsorbate for the corresponding pore with radius $r_{p}$. However assumptions clearly not fully valid for many materials (like rigid pores with regular pore shape, ideal meniscus contact angles $\left(0^{\circ}\right)$, filling/emptying independent of the position in the material, adsorption on the pore walls similar to an open surface) can only give rough estimates of the pore size distribution.

\section{$\mathrm{NH}_{3}$-TPD}

$\mathrm{NH}_{3}$-TPD was performed at Haldor Topsøe in Denmark. 150-300 mg zeolite was activated at $500^{\circ} \mathrm{C}$ under $\left(75 \mathrm{ml} / \mathrm{min}\right.$.) $\mathrm{N}_{2}$ flow for 1 hour before cooling to $150^{\circ} \mathrm{C}$. The sample was kept at this temperature for $30 \mathrm{~min}$. under $2 \% \mathrm{NH}_{3}$ in $\mathrm{He}(75 \mathrm{ml} / \mathrm{min}$.) To remove physisorbed $\mathrm{NH}_{3}$ the samples were left in $\mathrm{N}_{2}$ flow for $2 \mathrm{~h}(75 \mathrm{ml} / \mathrm{min}$.). The temperature was then increased with $10^{\circ} \mathrm{C} / \mathrm{min}$. under $\mathrm{N}_{2}$ flow $\left(75 \mathrm{ml} / \mathrm{min}\right.$.) to $740^{\circ} \mathrm{C}$. Desorbed $\mathrm{NH}_{3}$ was detected with mass spectrometry (MS). In the $\mathrm{Si} / \mathrm{Al}$ ratio calculation it was assumed that one $\mathrm{NH}_{3}$ molecule adsorbs per acid site. 


\section{Infrared Spectroscopy (IR)}

Spectroscopy is based on the interaction of matter with electromagnetic radiation. Dependent on the energy of the radiation different features of the material can be identified since a molecule can distribute its energy over several available energy states (translation, rotation, vibration, electron and core excitations) having energy levels with increasing separation going from translation to core excitations.[115, 116] The rather low energy infrared radiation typically fits with the vibrational (or rotational) movement of matter which means that any absorbed frequency of the incoming light will correspond to a vibration in the zeolite framework. These defined stretch and bending frequencies depend on the type of atoms, the bond strength and the influence of surrounding molecules (e.g. water in the pores of the zeolite or probe molecules). An approximate measure of the vibrations can be expressed by a simple harmonic oscillator (spring and ball model) given in formula 1 . The vibrational frequency is a function of the masses, $\mu$ (reduced mass), and the strength of the bond, $\mathrm{k}$ (force constant).[116]

$$
v=\frac{1}{2 \pi} \sqrt{\frac{k}{\mu}}
$$

This implies that bonds between heavy atoms result in low vibrational frequency while light elements give high vibrational frequency. It also implies that a shorter and stronger bond, represented by k, results in high(er) vibrational frequency while a weak bond will vibrate at low(er) frequency. Thus low acidic $\equiv \mathrm{Si}-\mathrm{OH}$ (terminal silanols) located on the external crystal surface is found at high frequencies $3745 \mathrm{~cm}^{-1}$. The stronger Brønsted acid site $(\equiv \mathrm{Si}-(\mathrm{OH})-\mathrm{Al} \equiv)$ is located at $3610 \mathrm{~cm}^{-1}$. The absorbance of defect related $\equiv$ Si$\mathrm{OH}$ groups depends on the surroundings. When $\mathrm{H}$ bonds are present the frequency is shifted to $3500 \mathrm{~cm}^{-1}$ while isolated $\equiv \mathrm{Si}-\mathrm{OH}$ only slightly shifts to lower frequencies.[117] Thus infrared spectroscopy can be used to identify the type of acid site, density and acid strength as well as crystal defects.[117] 


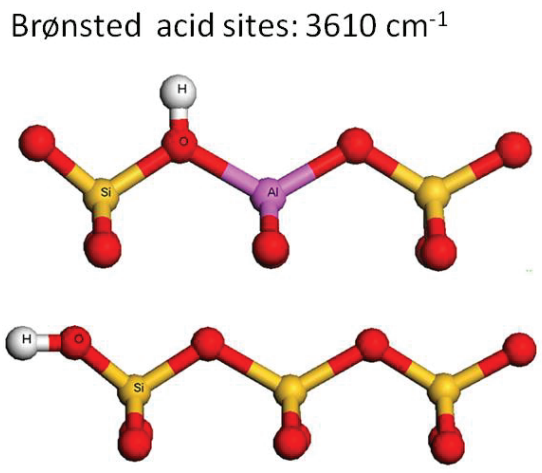

Terminal silanols: $3745 \mathrm{~cm}^{-1}$
Perturbed silanols ( $\mathrm{H}$ bond): $3500 \mathrm{~cm}^{-1}$

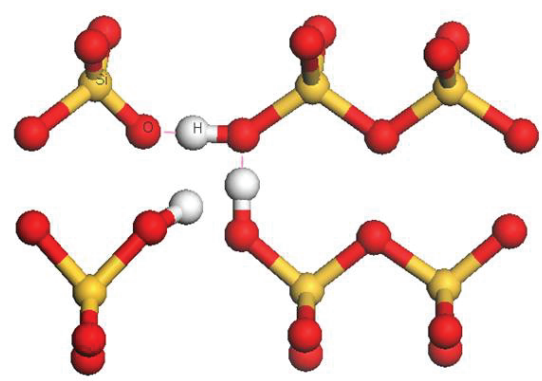

Isolated silanols: $3720 \mathrm{~cm}^{-1}$

Figure 37: Relevant frequencies of zeolites in FTIR. Figure modified from [117].

Additionally probe molecules such as $\mathrm{CO}$ are commonly used to investigate the acid sites in zeolites. The base interacts with the proton on the zeolite and the changes in the absorption spectra can be followed to identify the acid strength. Probe molecules with different sizes e. g. pyridine and collidine (2, 4, 6-trimethylpyridine) are often used to identify the accessibility to the zeolite pore system. For details on FTIR and probe molecules applied to zeolites it is referred to literature.[118]

In this work zeolite powder was pressed into thin wafers (or eventually dispersed in methanol and dried on a silicon wafer), put in gold envelope and heated under vacuum for 3 hours. The sample was kept at $120^{\circ} \mathrm{C}$ for $1 \mathrm{~h}, 350^{\circ} \mathrm{C}$ for $1 \mathrm{~h}$ and finally at $450^{\circ} \mathrm{C}$ for $1 \mathrm{~h}$ before it was cooled down under vacuum. Spectra were collected on a Vertex 80 with $\mathrm{MCT}$ detector by using $\mathrm{CO}$ as a probe molecule at liquid nitrogen temperature.

\section{Raman Spectroscopy}

\section{The Raman Effect:}

Quantum chemistry operates with well defined stationary states. Normally a molecule is likely to be in its ground stationary state, but may absorb energy to excite to the next stationary state. Absorption of energy to excite from one stationary (typically vibrational) state to another stationary state is what happens in IR. In some cases a molecule can gain energy which does not correspond to the difference in stationary states. It is much more unlikely to happen (and therefore a high intensity laser beam is often needed), but it may 
happen. In such a situation the molecule is in a highly energetic virtual state and will not remain in this unstable situation for a long time. From the virtual state the molecule can fall into many stationary states again sending out light with energy correspondent to the energy difference between the virtual state and the stationary state it falls into as shown in Figure 38 .

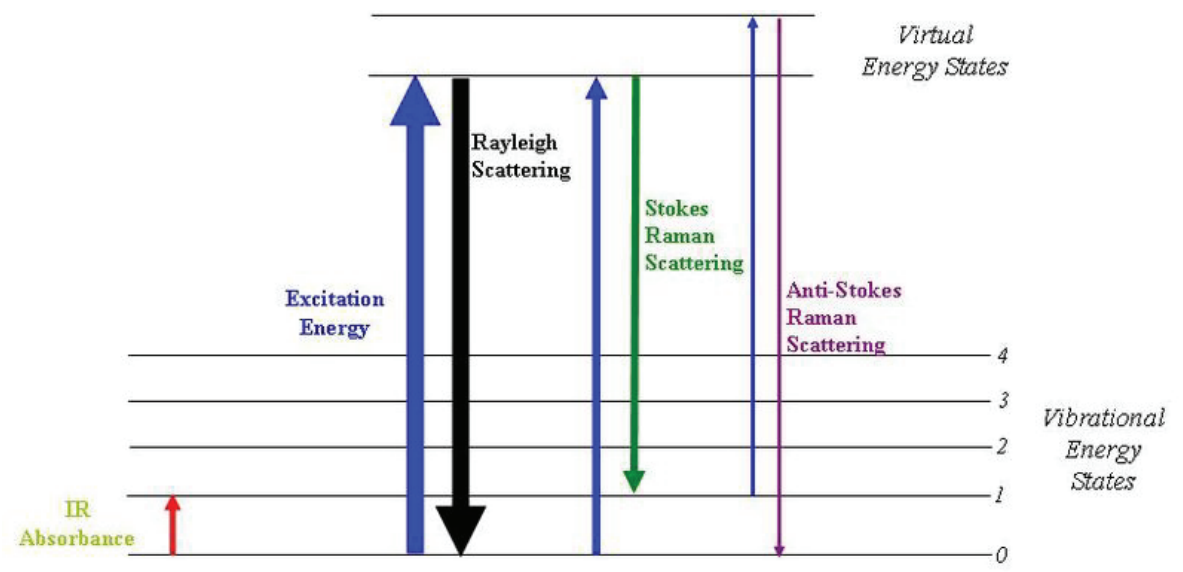

Figure 38: The Raman Effect. Figure adapted from [119].

In cases where the incoming light only polarizes the electron cloud around the nucleus the difference between the incoming light and the scattered light is small. This is an elastic process called Rayleigh scattering. When the scattering process involves a change in nuclear motion with energy transfer from the incoming photon to the molecule the scattered light will be of lower energy. This is called Stokes Raman scattering. In contrast, when the molecule transfers energy to the photon the scattered light will be of higher energy. Thus the latter process is called Anti-Stoke Raman Scattering.

Raman spectroscopy applied to zeolites can be used to investigate features of the inorganic framework, zeolites synthesis, adsorbed molecules and reactions, confined metal complexes etc.[120] Due to different selection rules than FTIR, Raman spectroscopy show bands where there are no dipole moments, opposite of FTIR. Hence the two methods are complementary.

Raman set up relevant to this work 
One problem with Raman spectroscopy is the high intensity of the laser light which may cause damage to the catalyst and result in heat gradients between the point of measurement and the catalyst bed, eventually affecting the ongoing chemistry. Operando Raman spectroscopy of zeolites converting methanol requires heat removal because methanol is known to transfer to coke by the Raman laser itself under reaction conditions far below temperatures where methanol normally is converted to hydrocarbons over the zeolites.[121] One way to avoid this problem is to reduce the contact time of the catalyst particles with the laser. Recently Beato et al. [121] presented a fluidized bed microreactor fitted for Raman spectroscopy dealing with the problems of heating and damage to the catalyst. The same reactor, Figure 39, has been applied in PAPER III to study coke formation over zeolites in MTH. Reactant gas flow is sent from the top to the bottom of the reactor and passes through the catalyst bed placed on a ceramic fiber filter in a ceramic sample holder on the way downwards. A downstream placed micro device gives back pulses to lift the particles, but since the net flow is downwards the catalyst is kept in the sample holder. The sample moves in a quasi-circular mode. More details can be found in $[121]$.
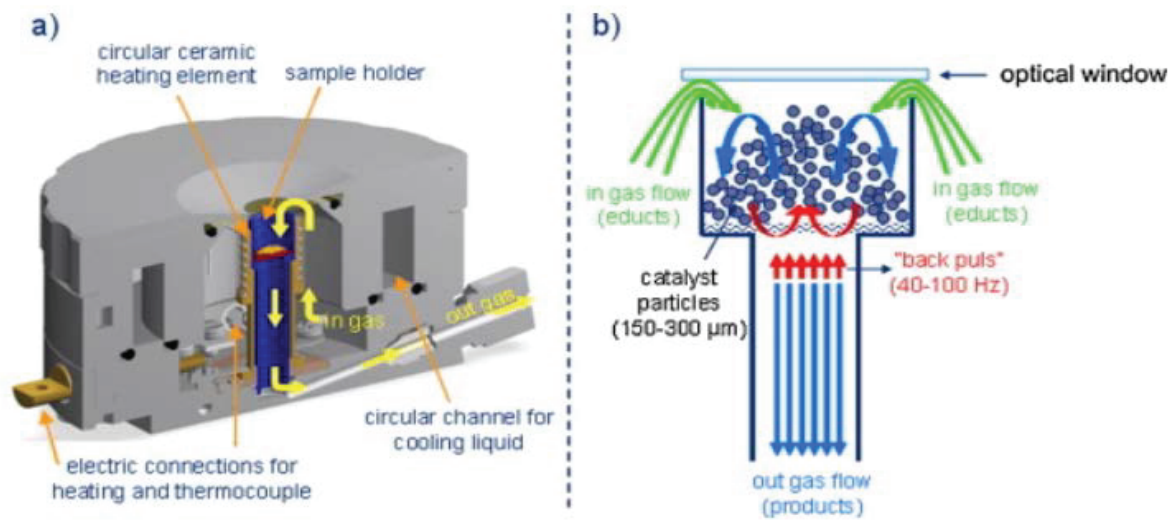

Figure 39: The micro-reactor (left panel) with the sample cell (right panel) employed in PAPER III. The figure is adopted from [121].

A system was developed to give defined pulses of methanol towards the microreactor as shown in Figure 40. 


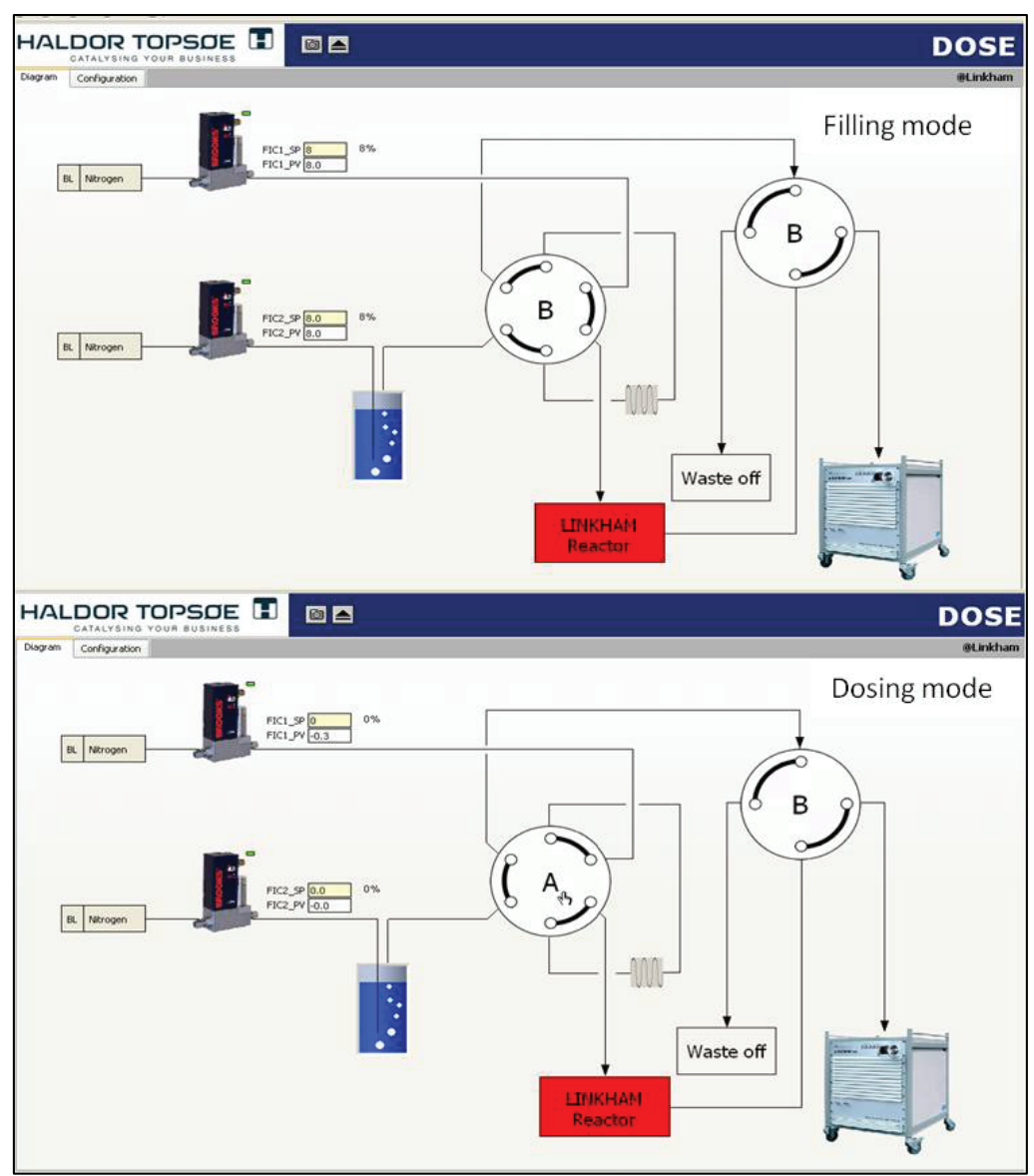

Figure 40: The methanol pulse dosing system. In filling mode the gas flow passes through the methanol saturator and further to the loop. Only inert gas passes through the reactor. In dosing mode the 6-port valve is switched and methanol filled in the loop is dosed towards the reactor where it converts to hydrocarbons which can be detected by MS or sent to waste.

Raman spectroscopy was carried out to follow the development of coke species in 3D zeolites in situ in methanol conversion. A Renishaw micro-Raman system 1000 with an $\mathrm{Ar}^{+}$laser operating at $244 \mathrm{~nm}$ (after doubled frequency) was used for the measurement. A $15 \times$ microscope objective was used to excite and collect the Raman signal. A monochromator with grating 3600 lines $/ \mathrm{mm}$ and a CCD camera was used in the measurement.

Prior to measurements the samples $(30 \mathrm{mg})$ were pretreated in air. The ramp was 
$10^{\circ} \mathrm{C} / \mathrm{min}$ from room temperature to $550^{\circ} \mathrm{C}$. At this temperature the sample was kept for 1 $\mathrm{h}$ and cooled to $350^{\circ} \mathrm{C}$ (reaction temperature) still in inert gas. The measurement was carried out in Ar flow with defined methanol pulses to the fluidized reactor described above with the laser beam analyzing the sample through a window on top after the required number of methanol pulses. Details on the experimental part are given in PAPER III.

\subsection{Post synthesis treatment}

\subsubsection{Alkaline treatment}

The samples were treated with $30 \mathrm{ml} 0.2-1.0 \mathrm{M} \mathrm{NaOH}$ solutions per gram of the respective zeolite and left for $0.5-1.5 \mathrm{~h}$ in a water bath at $70^{\circ} \mathrm{C}$. The solutions were then cooled in an ice bath, centrifuged and washed with distilled water until $\mathrm{pH}$ was close to neutral. The samples were dried before ion exchange in $1 \mathrm{M} \mathrm{NH}_{4} \mathrm{NO}_{3}$ solution for $3 \mathrm{x} 2 \mathrm{~h}$ at $70{ }^{\circ} \mathrm{C}$. Dried samples were again calcined.

\subsubsection{Combined alkaline and surfactant treatment}

Per gram zeolite $20 \mathrm{ml}$ of the combined $\mathrm{NaOH}$ and $\mathrm{CTAB}$ solution was added in a teflon liner in a stainless steel autoclave and put in a tumbling $(37 \mathrm{rpm})$ oven at $80^{\circ} \mathrm{C}$ for $24 \mathrm{~h}$. The samples quenched in an ice bath, centrifuged and washed several times with distilled water until $\mathrm{pH}$ was close to neutral. The samples were calcined at $550{ }^{\circ} \mathrm{C}$ for $8 \mathrm{~h}, 3 \mathrm{~h}$ to reach $550{ }^{\circ} \mathrm{C}$ and then held at this temperature for $5 \mathrm{~h}$, in a flow of $50: 50 \mathrm{~N}_{2}$ and $\mathrm{O}_{2}$. Calcined samples were ion exchanged in $1 \mathrm{M} \mathrm{NH}_{4} \mathrm{NO}_{3}$ solution for three times $2 \mathrm{~h}$ at 70 ${ }^{\circ} \mathrm{C}$. Dried samples were again calcined with the same treatment, except using air, to obtain the proton form. 


\subsection{Catalytic testing}

The experiments were carried out with methanol as the reactant in a reactor system presented in Figure 41.

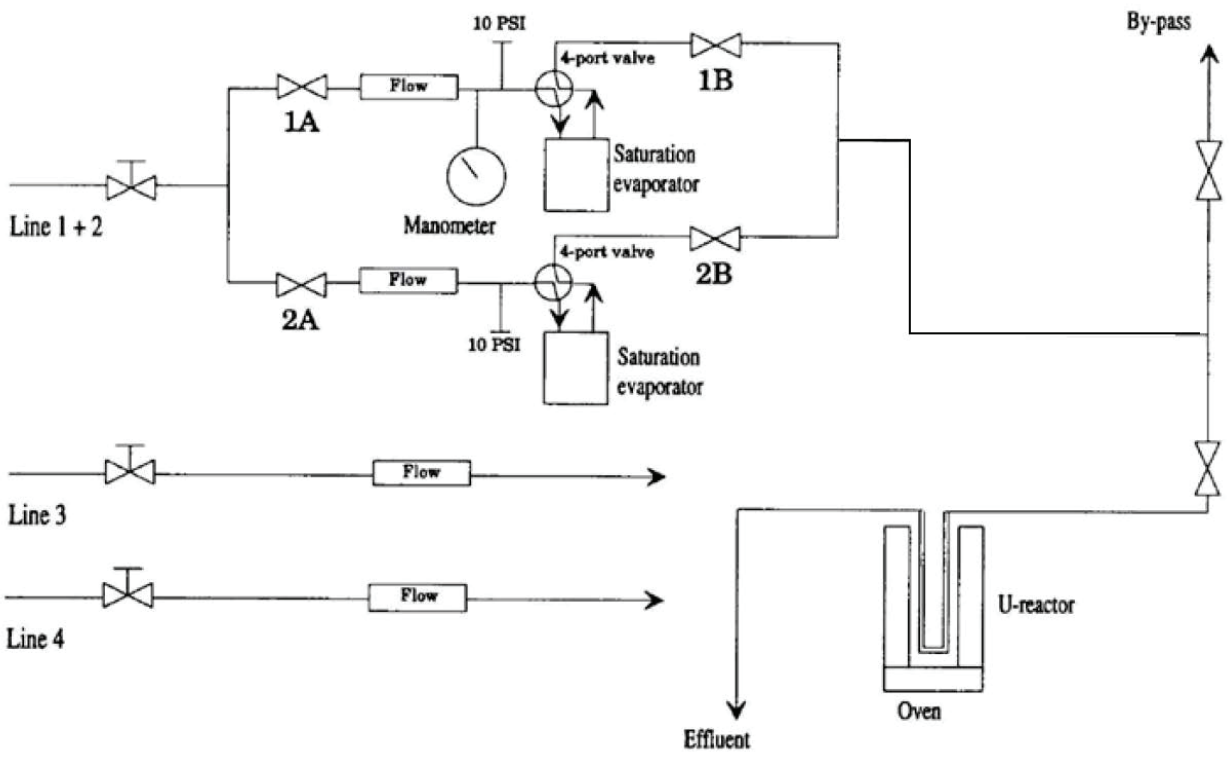

Figure 41: The reactor system for MTH experiments designed by Rønning [122].

The reactor system has four gas lines. Line 1 and 2 uses He as carrier gas and passes through a saturator containing liquid methanol (BDH Laboratory Supplies, > 99.8\% chemical purity). In ordinary experiments only Line 1 was used. (Line 2 may be used in e.g. switching experiments from ${ }^{12} \mathrm{C} \mathrm{MeOH}$ to ${ }^{13} \mathrm{C} \mathrm{MeOH}$ in mechanistic studied in MTH.) Line 3 is also connected to He while Line 4 is connected to $\mathrm{O}_{2}$ (allowing in situ calcination and regeneration). The gas flow was controlled by Porter P-150 ball flow meters and measured with ADM2000 Universal gas flow meter (Agilent Technologies). The carrier gas flow and the temperature of the water bath surrounding the methanol saturator was adjusted $\left(20^{\circ} \mathrm{C}\right)$ to obtain the wanted reactant flow rates (WHSV - weight hour space velocity). A thermocouple is mounted in the oven to monitor the oven temperature.

The catalytic tests were performed typically with $50 \mathrm{mg}$ sample in a fixed bed 
quartz reactor (with a thermocouple inserted to control the temperature at the bottom of the catalyst bed) with $6-10 \mathrm{~mm}$ inner diameter. The zeolite powder was pressed into wafers and subsequently crushed and sieved to obtain particles in the range 250-420 $\mu \mathrm{m}$ before transferring the catalyst to the reactor. Before each test the catalysts were calcined in situ in pure oxygen for $1 \mathrm{~h}$ at $550^{\circ} \mathrm{C}$ to remove eventual species adsorbed during handling. The product stream was analyzed using an automatic injection gas chromatograph connected directly to the reactor outlet by a heated transfer line. An Agilent 6890A GC with FID, equipped with a Supelco SPB-5 capillary column (60 m, $0.530 \mathrm{~mm}$ i.d. stationary phase thickness $3 \mu \mathrm{m}$ ) was used for the analysis. The temperature of the oven was programmed between 45 and $260^{\circ} \mathrm{C}$ with a heating rate of $25^{\circ} \mathrm{C} \mathrm{min}^{-1}$ (hold time $=5 \mathrm{~min}$. at $45^{\circ} \mathrm{C}$ and $16 \mathrm{~min}$. at $260^{\circ} \mathrm{C}$ ).

Entrapped species in the zeolite were analyzed after completed reaction on an offline GC-MS. Prior to analysis $20 \mathrm{mg}$ of coked zeolite was transferred to $1 \mathrm{ml}$ teflon vials before adding $1 \mathrm{ml} 15 \%$ HF (Fluka, $40 \%$ ). $1 \mathrm{ml}$ Dichloromethane (Merck, > 99.9 \%) with hexachloroethane (Supelco, $99.9 \%$ ) as internal standard was used for the extraction of the organic compounds. The organic phase was injected to an Agilent $6390 \mathrm{~N}$ gas chromatograph connected to an Agilent 5793 mass selective detector equipped with a HP-5MS column (60 m, $0.25 \mathrm{~mm}$ i.d., stationary phase thickness $0.25 \mu \mathrm{m})$. The temperature of the oven was programmed between 50 and $300^{\circ} \mathrm{C}$ with a ramp of $10^{\circ} \mathrm{C} / \mathrm{min}$ (hold time $=3 \mathrm{~min}$. at $50^{\circ} \mathrm{C}$ and $15 \mathrm{~min}$. at $300^{\circ} \mathrm{C}$ ). The NIST98 mass spectral library was used to determine the compounds analyzed. 


\section{Summary of results}

This work is concentrating on shape selective properties of zeolite catalysts (Paper I, II and III) and methods to investigate deactivation patterns in zeolite catalysts with in situ Raman spectroscopy (Paper IV). Post synthesis modifications, meant to improve lifetimes, of several zeolites have been carried out to investigate how the treatment affects the lifetime in MTH (Paper V). Hence the thesis deals with issues of shape selectivity deactivation and methods meant to improve life times in zeolites as catalysts.

Prior to the articles, manuscripts and preliminary results an extensive synthesis effort has been performed. Except of the commercial available catalysts (and some of the ZSM-22 samples) the zeolites were synthesized by the candidate with recipes described in the experimental section of this thesis. A brief summary of the synthesis results and characterisation will be presented prior to the presentation of the already published articles or manuscripts under review.

\subsection{Synthesis results and material characteristics}

\subsubsection{TNU-9 and IM-5}

\section{Synthesis aspects}

Many of the herein synthesised SDA's have low structure directing effect, and act more as pore fillers. Typically the low structure directing effect became visible varying the inorganic gel composition, as only small differences in many cases changed the phase. One of the most illustrating examples of this effect was TNU-9. In the case of TNU-9 with 1,4-MPB as a SDA several other phases (analcime, mordenite, quartz, ZSM-12) appeared when the $\mathrm{Si} / \mathrm{Al}$ ratio, the $\mathrm{NaOH} / \mathrm{SiO}_{2}$ and the crystallization conditions (time, static, stirring, tumbling) were varied. In the original paper [108] 9 different phases appeared when varying the $\mathrm{Si} / \mathrm{Al}$ ratio $(7.5-60$ and 0$)$, the $\mathrm{NaOH} / \mathrm{SiO}_{2}$ ratio $(0.47-1.00)$ and the crystallization conditions (time, static, stirring, tumbling). Despite trials of many gel compositions with 1,4-MPB as a template TNU-9 was synthesized only in a narrow range of gel compositions. Si/Al ratios between (slightly higher than) 15 and 30 and a fixed $\mathrm{NaOH} / \mathrm{SiO}_{2}$ ratio of 0.73 was the optimal composition of the synthesis mixture. 
These results are close to the original paper that also included $\mathrm{Si} / \mathrm{Al}=15$ [108], but in the herein presented results analcime was typically present to some degree at such high $\mathrm{Al}$ loadings. Trials to expand the $\mathrm{Si} / \mathrm{Al}$ ratio above 30 could result in TNU-9 with no other phases, but again the $\mathrm{Si} / \mathrm{Al}$ ratio in the crystals were literarily almost constant $(\sim 15)$. Often also other phases appeared. Lowering the $\mathrm{Si} / \mathrm{Al}$ ratio (equal or lower than 15 in the gel) typically resulted in analcime (and TNU-9).

\section{Could Ge incorporation help expanding the $\mathrm{Si} / \mathrm{Al}$ ratio in TNU-9?}

Why was the $\mathrm{Si} / \mathrm{Al}$ ratio so difficult to expand for this structure? One hypothesis was that the structure contained strained parts requiring bond angles facilitated by a high density of acid sites. Since several zeolites with strained structural units are stabilized with Ge, $\mathrm{GeO}_{2}$ were added to the synthesis mixture with relative molar ratios given in Table 14 . $\mathrm{The} \mathrm{Si} / \mathrm{Al}$ ratios in the synthesis mixture were then $18,26,37$ and 40.

Table 14: Synthesis conditions in relative molar ratios.

\begin{tabular}{cccccccc}
\hline $\mathbf{N a}_{2} \mathbf{O}$ & $\mathrm{Al}_{2} \mathbf{O}_{3}$ & $\mathbf{S i O}_{2}{ }^{a}$ & $\mathbf{G e O}_{2}$ & $\mathbf{R}$ & $\mathbf{H}_{2} \mathbf{O}$ & Time $^{\mathbf{c}}$ & Temperature \\
\hline 10,95 & $0,33-0,83$ & 30 & 3,00 & $4,5^{\mathrm{b}}$ & 1200 & 14 days & $160{ }^{\circ} \mathrm{C}$
\end{tabular}

a: Aerosil 200, b) R = 1,4-MPB, c) The crystallization was performed under tumbling conditions.

Starting with $\mathrm{Si} / \mathrm{Al}$ ratio 18 , but else optimal TNU-9 conditions, addition of $\mathrm{GeO}_{2}$ now instead led to NU-87 plus hydrated sodium germanium oxide. Increasing $\mathrm{Si} / \mathrm{Al}$ ratio gave a mixture of TNU-9 and NU-87. In addition to the Ge rich phase. Hence pure TNU-9 was not obtained and Ge did not help to expand the $\mathrm{Si} / \mathrm{Al}$ ratio under the described condition

\section{Introduction of $\mathrm{NaBr}$ to the synthesis mixture of TNU-9}

Attempts to investigate the influence if introduction of Br could lead to improved charge balance and hence increase the $\mathrm{Si} / \mathrm{Al}$ ratio above the reported values was carried out with the following gel compositions: $4.5(1,4-\mathrm{MPB}) \cdot 10,95 \mathrm{Na}_{2} \mathrm{O} \cdot 0.375-0.6 \mathrm{Al}_{2} \mathrm{O}_{3} \cdot 30 \mathrm{SiO}_{2}$. $1.2 \mathrm{NaBr} \cdot 1200 \mathrm{H}_{2} \mathrm{O}$. Despite that gel compositions with $\mathrm{Si} / \mathrm{Al}$ ratio at 40 also gave TNU-9 under tumbling conditions ( 10 days at $160^{\circ} \mathrm{C}$ ) EDS indicated that despite lowering the amount of $\mathrm{Al}$ the resulting $\mathrm{Si} / \mathrm{Al}$ ratio in the particles were only changed to a minor degree (raised from 14 to 18), confirming the very narrow range of compositions. Similar 
compositions, but static crystallization conditions led to other phases. Hence under the investigated conditions neither $\mathrm{Ge}$ incorporation nor adding $\mathrm{NaBr}$ facilitated increased $\mathrm{Si} / \mathrm{Al}$ ratios.

\section{Rapidly reduction of crystallization conditions}

Both in the herein obtained results and in literature [108] very long crystallization times were required to obtain TNU-9 under static conditions. As long as 27 days were needed to crystallize TNU-9 at $160^{\circ} \mathrm{C}$ under static conditions, but the structure appeared at shorter crystallization times when stirring was applied. Even shorter crystallization times $(\sim 10$ days) were needed to obtain highly crystalline TNU-9 if the synthesis mixture was tumbled (37 rpm).

\section{Summary of the synthesis conditions of TNU-9 and obtained products}

Summary of synthesis conditions based on the herein obtained results and literature [108] is given in Table 15 .

Table 15: Overview of synthesis conditions and resulting product.

\begin{tabular}{|c|c|c|c|c|c|}
\hline \multirow[t]{2}{*}{ Run } & \multicolumn{3}{|c|}{ Gel composition $^{\mathrm{a}}$} & \multirow[t]{2}{*}{ Crystallization conditions } & \multirow[t]{2}{*}{ Product $^{c}$} \\
\hline & $\mathrm{Si} / \mathrm{Al}$ & $\mathrm{NaOH} / \mathrm{SiO}_{2}$ & $\mathrm{~F}^{-} / \mathrm{Br}^{-} / \mathrm{Ge}$ & & \\
\hline $1^{\mathrm{b}}$ & 15 & 0.73 & - & Static (27d) & TNU-9 (+ ANA) \\
\hline $2^{b}$ & 20 & 0.73 & - & Static (27d) & TNU-9 \\
\hline $3^{b}$ & 25 & 0.73 & - & Static (27d) & TNU-9 \\
\hline $4^{c}$ & 22 & 0.73 & - & Tumbling (12 d) & TNU-9 \\
\hline $5^{c}$ & 25 & 0.73 & - & Tumbling (12 d) & TNU-9 \\
\hline $6^{\mathrm{c}}$ & 31 & 0.73 & - & Tumbling (12 d) & TNU-9 \\
\hline 7 & 35 & 0.73 & - & Tumbling $(14 \mathrm{~d})$ & TNU-9 \\
\hline 8 & 50 & 0.73 & - & Tumbling (14 d) & Quartz (+ MOR) \\
\hline 9 & 15 & 0.73 & - & Tumbling (10 d) & TNU-9 (+ ANA) \\
\hline 10 & 20 & 0.73 & - & Tumbling (10 d) & TNU-9 \\
\hline 11 & 25 & 0.73 & - & Tumbling $(10 \mathrm{~d})$ & TNU-9 \\
\hline 12 & 30 & 0.73 & - & Tumbling (10 d) & TNU-9 \\
\hline 13 & 25 & 0.73 & - & Stirring (18 d) & TNU-9 \\
\hline $14^{\mathrm{c}}$ & 25 & 0.73 & - & Stirring (12 d) & TNU-9 \\
\hline $15^{\mathrm{d}}$ & $\begin{array}{c}25,35 \\
40\end{array}$ & 0.73 & $\mathrm{Br}^{-}$ & Stirring (18 d) & TNU-9 \\
\hline 16 & 18 & 0.73 & $\mathrm{Ge}$ & Tumbling (14 d) & $\mathrm{NU}-87+?$ \\
\hline 17 & $\begin{array}{c}26,37 \\
45\end{array}$ & 0.73 & $\mathrm{Ge}$ & Tumbling (14 d) & $\begin{array}{l}\text { NU-87 + TNU-9 + } \\
?\end{array}$ \\
\hline 18 & $25-50$ & 0.55 & $\mathrm{Br}^{-}$ & Static $(10 \mathrm{~d})$ & ZSM-12 (+ quartz) \\
\hline 19 & $33-50$ & 0.73 & $\mathrm{Br}^{-}$ & Static $(10 \mathrm{~d})$ & $\begin{array}{l}\text { Unknown phase }+ \\
\text { (mordenite and }\end{array}$ \\
\hline
\end{tabular}


quartz at higher

$\mathrm{Si} / \mathrm{Al})$

\begin{tabular}{|c|c|c|c|c|}
\hline \multicolumn{5}{|c|}{ Literature Hong et. al. [108] } \\
\hline 1 & 30 & 0.73 & Rotation (14 d) & TNU-9 \\
\hline 2 & 30 & 0.73 & Static & Amorphous \\
\hline 3 & 7.5 & 0.73 & Rotation (14 d) & ANA + (TNU-9) \\
\hline 4 & 15 & 0.73 & Rotation (14 d) & MOR + TNU-9 \\
\hline 5 & 20 & 0.73 & Rotation (14 d) & TNU-9 \\
\hline 6 & 60 & 0.73 & Rotation (14 d) & Quartz + (TNU-9) \\
\hline 7 & $\infty$ & 0.73 & Rotation (14 d) & MCM-47 \\
\hline $8^{\mathrm{e}}$ & $\infty$ & 0.73 & Rotation (14 d) & MCM-47 \\
\hline 9 & 30 & 1.00 & Rotation (14 d) & TNU-10 \\
\hline 10 & 30 & 0.87 & Rotation (14 d) & TNU-9 + IM-5 \\
\hline 11 & 30 & 0.60 & Rotation (14 d) & ZSM-12 \\
\hline 12 & 30 & 0.47 & Rotation (14 d) & ZSM-12 \\
\hline 13 & 30 & $0.73^{f}$ & Rotation (14 d) & $\mathrm{D}^{\mathrm{g}}+(\mathrm{ZSM}-12)$ \\
\hline 14 & 30 & $0.73^{\mathrm{h}}$ & Rotation (14 d) & Amorphous \\
\hline $15^{\mathrm{i}}$ & 30 & 0.73 & Rotation (10 d) & TNU-9 \\
\hline $16^{\mathrm{j}}$ & 30 & 0.73 & Rotation (6d) & TNU-9 \\
\hline
\end{tabular}

${ }^{\mathrm{a}}$ The standard gel composition was $4.5(1,4-\mathrm{MPB}) \cdot \mathrm{xNa}_{2} \mathrm{O} \cdot \mathrm{yAl}_{2} \mathrm{O}_{3} \cdot 30 \mathrm{SiO}_{2} \cdot 1200 \mathrm{H}_{2} \mathrm{O}$, where $\mathrm{x}$ and y varied between $7,0 \leq \mathrm{x} \leq 15,0$ and $0,0 \leq \mathrm{y} \leq 2,0$ (temperature during crystallization: $160^{\circ} \mathrm{C}$ ), ${ }^{\mathrm{b}}:$ : Cabosil, ${ }^{\mathrm{c}}$ : In situ made template with composition $4.5(1,4-\mathrm{DBB}) \cdot 9.0(1-\mathrm{MP}) \cdot 11 \mathrm{Na}_{2} \mathrm{O} \cdot \mathrm{yAl}_{2} \mathrm{O}_{3} \cdot 30 \mathrm{SiO}_{2} \cdot 1200 \mathrm{H}_{2} \mathrm{O},{ }^{\mathrm{d}}: 4.5(1,4-$ MPB) $\cdot 10,95 \mathrm{Na}_{2} \mathrm{O} \cdot 0.375-0.6 \mathrm{Al}_{2} \mathrm{O}_{3} \cdot 30 \mathrm{SiO}_{2} \cdot 1.2 \mathrm{NaBr} \cdot 1200 \mathrm{H}_{2} \mathrm{O},{ }^{\mathrm{e}}$ adding 2 wt- $\%$ silica from TNU-9 seeds, ${ }^{\mathrm{f}}$ $\mathrm{LiOH} / \mathrm{SiO}_{2}$ ratio, ${ }^{\mathrm{g}} \mathrm{D}$ is an unknown and likely a dense phase, ${ }^{\mathrm{h}} \mathrm{KOH} / \mathrm{SiO}_{2}$ ratio, ${ }^{\mathrm{i}} \mathrm{Gel}$ composition: 4.5(1,4DBB) $\cdot 9.0(1-\mathrm{MP}) \cdot 11 \mathrm{Na}_{2} \mathrm{O} \cdot 0.5 \mathrm{Al}_{2} \mathrm{O}_{3} \cdot 30 \mathrm{SiO}_{2} \cdot 1200 \mathrm{H}_{2} \mathrm{O}$ at $433 \mathrm{~K} . .{ }^{\mathrm{j}} \mathrm{Gel}$ composition: $4.5(1,4-\mathrm{DBB}) \cdot 13.5(1-$ $\mathrm{MP}) \cdot 11 \mathrm{Na}_{2} \mathrm{O} \cdot 0.5 \mathrm{Al}_{2} \mathrm{O}_{3} \cdot 30 \mathrm{SiO}_{2} \cdot 1200 \mathrm{H}_{2} \mathrm{O}$ ved $433 \mathrm{~K}$ i 6 days.

The optimal synthesis conditions are given in Table 16.

Table 16: Optimal synthesis conditions in relative molar ratios - TNU-9

\begin{tabular}{cccccccc}
\hline $\mathbf{N a}_{2} \mathbf{O}$ & $\mathbf{A l}_{2} \mathbf{O}_{3}$ & $\mathbf{S i O}_{2}$ & & $\mathbf{1 , 4 - M P B}$ & $\mathbf{H}_{2} \mathbf{O}$ & Time & Temperature \\
\hline 10.95 & $0.5-1.0$ & 30 & & 4.5 & 1200 & 10 days $^{\mathrm{a}}$ & $160{ }^{\circ} \mathrm{C}$ \\
\hline $\mathbf{N a}_{2} \mathbf{O}$ & $\mathbf{A l}_{2} \mathbf{O}_{3}$ & $\mathrm{SiO}_{2}$ & $\mathbf{1 - M P}$ & $\mathbf{1 , 4 - D B B}$ & $\mathbf{H}_{2} \mathbf{O}$ & Time & Temperature \\
\hline 10.95 & 0.5 & 30 & 13.5 & 4.5 & 1200 & 6 days & $160^{\circ} \mathrm{C}$
\end{tabular}

${ }^{\mathrm{a}}$ tumbling, ${ }^{\mathrm{b}}$ stirring (100 rpm), recipe from [108].

\section{The IM-5 case}

The very similar nature of IM-5 compared with TNU-9 was easily recognizable after the investigations carried out prior to catalytic testing and from literature review.[22, 123, 124] The gel composition and SDA (1,5 MPP) are close to identical. They differ with respect to the SDA as the chain expands by one $\mathrm{CH}_{2}$-group. Also here a very narrow range of synthesis conditions were required. At comparable synthesis conditions, slight deviations gave many similar phases as in the TNU-9 case. Low $\mathrm{Si} / \mathrm{Al}$ ratio led to 
analcime in addition to IM-5. Other common phases by changes in the Si/Al ratio, $\mathrm{NaOH} / \mathrm{SiO}_{2}$ ratio and crystallization conditions were mordenite, quartz and gmelinite. In [22] also ZSM-12 and an unknown layered material was registered. Changing from $\mathrm{NaOH}$ to $\mathrm{LiOH}$ or $\mathrm{KOH}$ TNU-9 was not observed in [22]. The crystallization time was also in this case reduced by using tumbling conditions compared to static crystallization. Table 17 gives an overview of the herein obtained results and results from literature.[22]

Table 17: Overview of synthesis conditions and resulting product.

\begin{tabular}{|c|c|c|c|c|c|}
\hline \multirow[t]{2}{*}{ Run } & \multicolumn{3}{|c|}{ Gel composition $^{\text {a }}$} & \multirow[t]{2}{*}{ Crystallization conditions } & \multirow[t]{2}{*}{ Product $^{b}$} \\
\hline & $\mathrm{Si} / \mathrm{Al}$ & $\mathrm{NaOH} / \mathrm{SiO}_{2}$ & $\mathrm{~F}^{-} / \mathrm{Br}^{-} / \mathrm{Ge}$ & & \\
\hline 1 & 20 & 0.73 & - & Static (27d) & IM-5 + ANA \\
\hline 2 & 25 & 0.73 & - & Static (27d) & IM-5 \\
\hline 3 & 30 & 0.73 & - & Static (27d) & IM-5 \\
\hline $4^{\mathrm{c}}$ & 20 & 0.73 & $\mathrm{Br}^{-}$ & Static (27 d) & $\mathrm{MOR}+\mathrm{IM}-5+?$ \\
\hline $5^{c}$ & $25-35$ & 0.73 & $\mathrm{Br}^{-}$ & Static (27 d) & IM-5 \\
\hline 8 & 15 & 0.73 & - & Tumbling (14 d) & $\mathrm{IM}-5+$ ANA \\
\hline 9 & $25-45$ & 0.73 & - & Tumbling (14 d) & IM-5 \\
\hline $10^{\mathrm{d}}$ & 35 & 0.73 & - & Stirring $(16 \mathrm{~d})$ & IM-5 \\
\hline 13 & 15 & 0.57 & - & Static $(10 \mathrm{~d})$ & Quartz + IM-5 \\
\hline 14 & 20 & 0.57 & - & Static (10 d) & GME + ANA? \\
\hline 15 & 25 & 0.57 & - & Static (10 d) & $\begin{array}{l}\text { Quartz+IM- } \\
5 \text { (MOR) }\end{array}$ \\
\hline 16 & 30 & 0.57 & - & Static (10 d) & Quartz + MOR \\
\hline 17 & 25 & 0.50 & - & Static (10 d) & Quartz + IM-5 \\
\hline 18 & 25 & 0.6 & - & Static (10 d) & $\mathrm{ANA}+\mathrm{MOR}$ \\
\hline 20 & 25 & 0.8 & - & Static (10 d) & IM-5 (+ MOR) \\
\hline 20 & 25 & 0.9 & - & Static $(10 \mathrm{~d})$ & ANA \\
\hline
\end{tabular}

\begin{tabular}{|c|c|c|c|c|}
\hline \multicolumn{5}{|c|}{ Literature Lee et. al. [22] } \\
\hline 1 & 30 & 0.73 & Rotation (14 d) & IM-5 \\
\hline 2 & 7.5 & 0.73 & Rotation (14 d) & $\mathrm{ANA}+\mathrm{IM}-5$ \\
\hline 3 & 20 & 0.73 & Rotation (14 d) & IM-5 \\
\hline 4 & 60 & 0.73 & Rotation $(14 \mathrm{~d})$ & IM-5 \\
\hline 5 & 120 & 0.73 & Rotation (14 d) & IM-5 \\
\hline 6 & $\infty$ & 0.73 & Rotation (14 d) & MOR \\
\hline 7 & 30 & 0.73 & Rotation (14 d) & $\mathrm{L}^{\mathrm{e}}$ \\
\hline 8 & 30 & 1.00 & Rotation (14 d) & ANA \\
\hline 9 & 30 & 0.87 & Rotation (14 d) & $\mathrm{MOR}+\mathrm{ANA}$ \\
\hline 10 & 30 & 0.60 & Rotation (14 d) & ANA + IM-5 \\
\hline 11 & 30 & 0.47 & Rotation (14 d) & ZSM-12 \\
\hline 12 & 30 & 0.33 & Rotation (14 d) & ZSM-12 \\
\hline 13 & 30 & $0.73^{\mathrm{f}}$ & Rotation (14 d) & ZSM-12 \\
\hline 14 & 30 & $0.73^{\mathrm{g}}$ & Rotation (14 d) & MOR \\
\hline
\end{tabular}

${ }^{\mathrm{a}}$ The general oxide composition was 4.5R: $x \mathrm{Na}_{2} \mathrm{O}: y \mathrm{Al}_{2} \mathrm{O}_{3}: 30 \mathrm{SiO}_{2}: 1200 \mathrm{H}_{2} \mathrm{O}$, where $\mathrm{R}=1,5-\mathrm{MPP}, \mathrm{x}$ varies in the range $5.0-15.0 \mathrm{og}$ y varies between 0 and 2.0 (temperature during crystallization: $160^{\circ} \mathrm{C}$ ), ${ }^{\mathrm{b}} \mathrm{The}$ phase given first was the dominating phase, ${ }^{\mathrm{c}}$ Composition: 5.0 1,5-MPP: $10.95 \mathrm{Na}_{2} \mathrm{O}: y \mathrm{Al}_{2} \mathrm{O}_{3}: 3.0 \mathrm{NaBr}$ : 
$30 \mathrm{SiO}_{2}: 1200 \mathrm{H}_{2} \mathrm{O}\left(175^{\circ} \mathrm{C}\right)$ - low yield. ${ }^{\mathrm{d}}$ In situ template formation and composition: 4.5(1,5-DBP) $13.5(1-$ $\mathrm{MP}) \cdot 10.95 \mathrm{Na}_{2} \mathrm{O} \cdot 0.16 \mathrm{Al}_{2} \mathrm{O}_{3} \cdot 30 \mathrm{SiO}_{2} \cdot 1200 \mathrm{H}_{2} \mathrm{O}$. ${ }^{\mathrm{e}}$ Unknown (likely) layered phase, ${ }^{\mathrm{f}} \mathrm{LiOH} / \mathrm{SiO}_{2},{ }^{\mathrm{g}} \mathrm{KOH} / \mathrm{SiO}_{2}$.

Based on the herein obtained results and from literature Table 18 gives the optimal synthesis conditions using 1,5-MPP as a SDA.

Table 18: Optimal synthesis conditions in relative molar ratios - IM-5.

\begin{tabular}{lcccccc}
\hline $\mathbf{N a}_{2} \mathbf{O}$ & $\mathbf{A l}_{2} \mathbf{O}_{3}$ & $\mathbf{S i O}_{2}$ & $\mathbf{1 , 5}-\mathbf{M P P}$ & $\mathbf{H}_{2} \mathbf{O}$ & Time & Temperature \\
\hline 10.95 & $0.5-1.00$ & 30 & 4.5 & 1200 & 10 days $^{\mathrm{a}}$ & $160{ }^{\circ} \mathrm{C}$ \\
& & & & & & \\
\hline${ }^{a}$ tumbling & & & & & &
\end{tabular}

\section{Material characteristics}

Results from representative synthesis procedures are shown in Figure 42. The XRD patterns shown are representative of pure phases of TNU-9 and IM-5. The coloured graphs were calculated from the theoretical unit cell in materials studio for comparison. All synthesis recipes of TNU-9 and IM-5 yielded small crystals. TNU-9 gave rod like crystals while IM-5 gave plate-rod-like crystals. The crystals may vary slightly in size, but were typically $<1 \mu \mathrm{m}$.

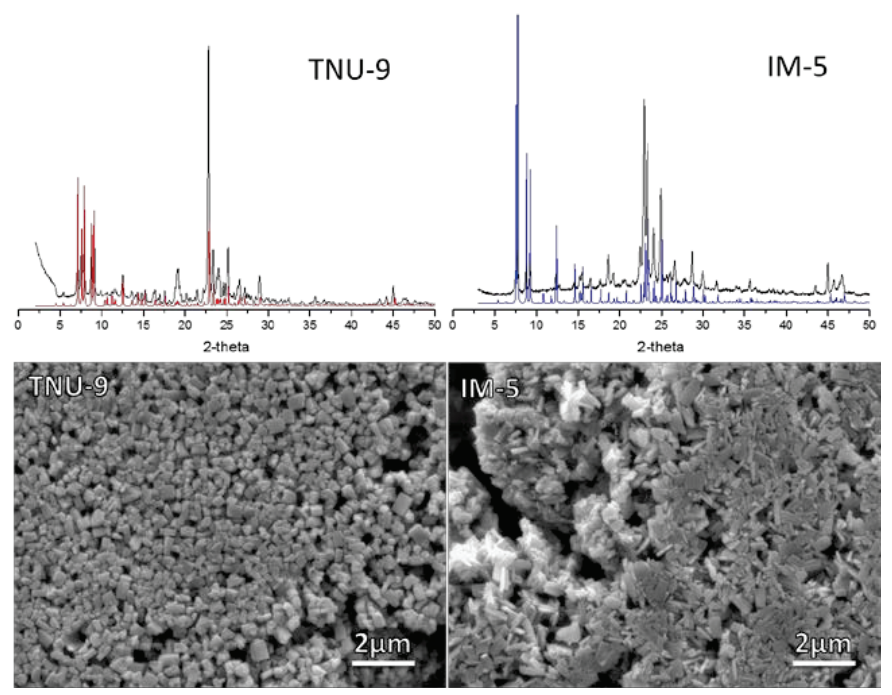

Figure 42: Representative XRD patterns and SEM micrographs. TNU-9 is shown in the left panel and IM-5 in the right panel. Colored graphs are calculated diffraction patterns in material studio. 
In the case of increasing the $\mathrm{Si} / \mathrm{Al}$ ratio in the TNU-9 synthesis mixture in the presence of $\mathrm{NaBr}$ the crystals increased in size (see Figure 43) . From rod-like crystals of size $1.5 \mu \mathrm{m}$ $\cdot 400 \mathrm{~nm}(\mathrm{Si} / \mathrm{Al}=25)$ to thicker rods $2 \mu \mathrm{m} \cdot 800 \mathrm{~nm}(\mathrm{Si} / \mathrm{Al}=35)$.

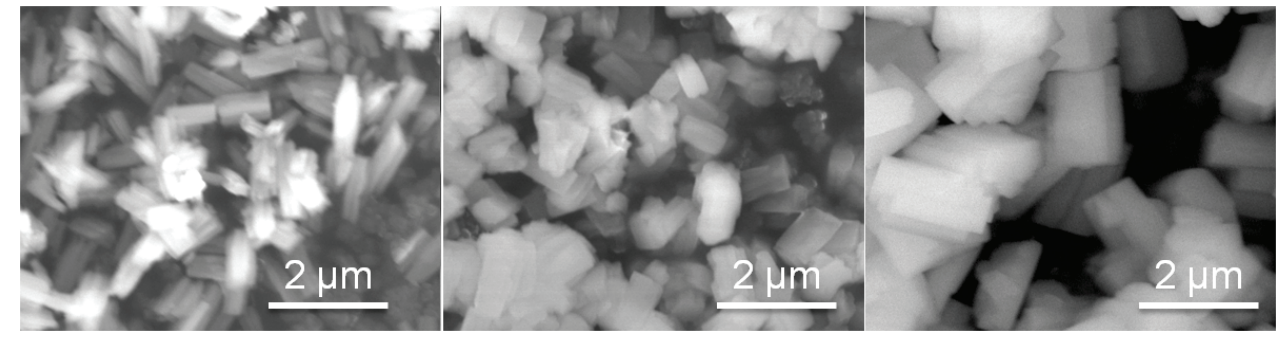

Figure 43: TNU-9 syntheses with $\mathrm{NaBr}$ tended to increase the crystal size when increasing the Si/Al ratio in the synthesis mixture. The Si/Al ratio was 25 (left panel), 30 (mid panel) and 35 (right panel).

Representative surface areas and acid site densities is given in Table 19. Both structures had a specific surface area well exceeding $400 \mathrm{~m}^{2} / \mathrm{g}$. As mentioned above, despite differences in gel compositions all measured crystals had $\mathrm{Si} / \mathrm{Al}$ ratio at $\sim 15$.

Table 19: Representative acid site density and surface area.

\begin{tabular}{cccccc}
\hline Structure & $\mathrm{Si} / \mathrm{Al}_{\mathrm{Gel}}$ & $\begin{array}{c}\mathrm{Si} / \mathrm{Al}_{\mathrm{EDS}} \\
(\text { Crystals })\end{array}$ & $\mathrm{Si} / \mathrm{Al}_{\mathrm{TPD}}$ & Crystals & $\begin{array}{c}\text { Surface } \operatorname{area} \\
{\left[\mathrm{m}^{2} / \mathrm{g}\right]}\end{array}$ \\
\hline TNU-9 & 25 & 16 & 15.9 & Rods typically $<1 \mu \mathrm{m}$ & 468 \\
IM-5 & 30 & 15 & - & Rods-plate $<1 \mu \mathrm{m}$ & 425 \\
\hline
\end{tabular}

\section{Suggestions for further work:}

Fluoride route: Only few attempts using the fluoride route were carried out to investigate whether the very narrow compositions of TNU-9 was a result of charge imbalance. Hence 1,4-MPB or $\mathrm{PMBr}_{2}$ where ion-exchanged to obtain the $1,4-\mathrm{MP}(\mathrm{OH})_{2}$ or the $\mathrm{PM}(\mathrm{OH})_{2}$. This method may allow removal of $\mathrm{NaOH}$ and charge balance of the positive charge on the template may be reached by introducing HF. In the few attempts the water amount was drastically reduced to increase the template concentration, but none of the attempts led to crystalline phases. The studies were initiated, but not completed as the risk of failure was estimated as too high within the time limitation. 
Alternative SDA: Since Hong et al. [108] found a drastic reduction of the crystallization time of TNU-9 when using in situ formed template at other stoichiometry ratios (3:1 1-MP: 1,4-DBB) than pre-forming the 1,4-MPB, this indicate that this SDA is not optimal stabilizing the structure. Supported by NMR Hong et al. claimed that most SDAs were intact, but that some decompose to fit into the zigzag channel (along the $\mathrm{x}$ axis) connecting two small channels (see material description in section 2.3.3). Both 1MP and methylamine (MA) was presented as possible organic species fitting into these areas of the pore system. Hence there should be a potential to find alternative SDAs or find other organic molecules which could act as co-template to 1,4-MPB. Recalling the work on Kubota et al. [18] a C/N $\mathrm{N}^{+}$of 11-15 was optimal for obtaining high silica phases by the $\mathrm{OH}^{-}$route while $1,4-\mathrm{MPB}$ has a $\mathrm{C} / \mathrm{N}^{+}$ratio of 7 . It is not straightforward to change the SDA keeping the similar structure, but it would be interesting to find a SDA with (slightly) different $\mathrm{C} / \mathrm{N}^{+}$and investigate differences in gel compositions. The introduction of smaller species such as MA or 1-MP would be of interest.

A similar conclusion is also valid for IM-5. Also here other SDAs would have been of interest to elucidate the effect of gel compositions on the $\mathrm{Si} / \mathrm{Al}$ ratio in the crystals.

\subsubsection{ZSM-23, ZSM-48, EU-1 (and ZSM-22)}

\section{Synthesis aspects}

\section{Me $_{6}$-diquat-n: ZSM-23, ZSM-48 and EU-1}

In the case of TNU-9 and IM-5 the very narrow range of gel compositions was an obstacle hindering flexibility in the elemental composition of the crystals. On the other hand when the SDA has a low structure directing effect this may also lead to a variety of structures. The synthesis of ZSM-23, ZSM-48 and EU-1 were appearing as a result of this effect since all of them may be synthesized by diquaternary alkylammonium ions (described as $\left(\mathrm{CH}_{3}\right)_{3} \mathrm{~N}^{+}\left(\mathrm{CH}_{2}\right)_{n} \mathrm{~N}^{+}\left(\mathrm{CH}_{3}\right)_{3}$ or $\mathrm{Me}_{6}$-diquat-n in [20]). The very similar nature of the SDAs tend to respond to changes in the gel compositions in much similar ways (e.g. phase produced). In Figure 44 the three SDAs used to synthesize EU-1 $\left(\mathrm{PMBr}_{2}\right), \mathrm{ZSM}-48\left(\mathrm{HexMBr}_{2}\right)$ and ZSM-23 $\left(\mathrm{HepMBr}_{2}\right)$ are shown. Although these SDAs were used to synthesize the three structures several other structures can be synthesized by 
changing the $\mathrm{Si} / \mathrm{Al}$ ratio or $\mathrm{NaOH} / \mathrm{SiO}_{2}$ ratio. (It should here be mentioned that the synthesis of the these structures is not limited to the SDAs herein presented. E.g. in the case of ZSM-23 it may be synthesized both with hepMBr $2, D M F$ hydrolyzing to dimethylamine (DMA) [125], ethylamine, isopropylamine, ethylenediamine or pyrolidine.[126, 127] In the case of ZSM-48 at least 13 different SDAs have been used to synthesize the structure.[13] Also ZSM-22 may be synthesized by several SDAs.

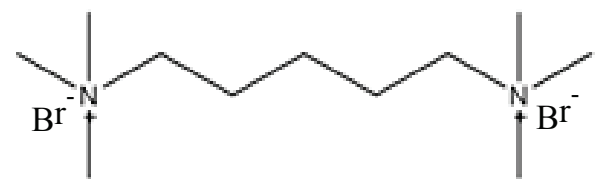

Pentamethonium bromide

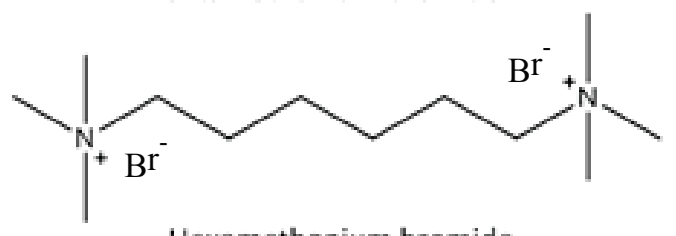

Hexamethonium bromide

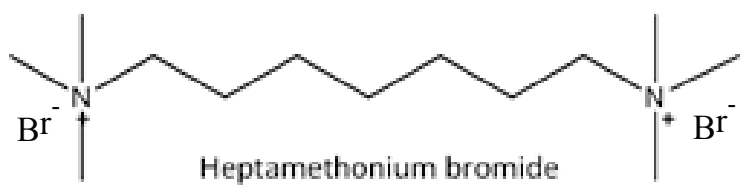

Figure 44: SDA producing EU-1, ZSM-48 and ZSM-23. Penthamethonium bromide = $\mathrm{PMBr}_{2}, \mathrm{Hexamethonium} \mathrm{bromide}=\mathrm{HMBr}_{2}$ and Heptamethonium bromide $=\mathrm{HepMBr}_{2}$. The positive charge is compensated by $\mathrm{Br}^{-}$.

Because of the difficulties trying other synthesis strategies to expand the $\mathrm{Si} / \mathrm{Al}$ ratio in TNU-9 and IM-5, it was focused on producing the 1D materials from synthesis compositions close to established recipes. Table 20, reprinted from [20], with slight modifications was used as a starting point for the syntheses given in the experimental synthesis section.

Table 20: Table reprinted from [20]

\begin{tabular}{lllll}
\hline Organic SDA & $3.0 \mathrm{R}: \mathrm{xNa}_{2} \mathrm{O}: \mathrm{yAl}_{2} \mathrm{O}_{3}: 30 \mathrm{SiO}_{2}: 1200 \mathrm{H}_{2} \mathrm{O}^{\mathrm{a} ; \mathrm{b}}$ & \\
& \\
& $\mathrm{x}=5.0$ \\
& $\mathrm{Si} / \mathrm{Al}$ in the synthesis mixture & & \\
\cline { 2 - 5 } & 30 & 60 & 120 & $\infty$ \\
\hline $\mathrm{Me}_{6}$-diquat-3 & Amorphous & Nonasil & & \\
& & & ZSM-48 & ZSM-48
\end{tabular}




$\begin{array}{lllll}\mathrm{Me}_{6} \text {-diquat-4 } & \text { Amorphous }^{\mathrm{c}} & \text { ZSM-12 } & \text { Amorphous } & \text { L } \\ \mathrm{Me}_{6} \text {-diquat-5 } & \text { EU-1 }^{\mathrm{c}} & \text { ZSM-48 } & \text { ZSM-48 } & \text { ZSM-48 } \\ \mathrm{Me}_{6} \text {-diquat-6 } & \text { Amorphous }^{\mathrm{c}} & \text { EU-1 } & \text { EU-1+ ZSM-48 } & \text { ZSM-48 } \\ \mathrm{Me}_{6} \text {-diquat-7 } & \text { Amorphous }^{\mathrm{c}} & \text { ZSM-23 } & \text { ZSM-23 } & \text { L } \\ \mathrm{Me}_{6} \text {-diquat-8 } & \text { Amorphous }^{\mathrm{c}} & \text { ZSM-5 } & \text { ZSM-5 } & \text { ZSM-48 } \\ \mathrm{Me}_{6} \text {-diquat-9 } & \text { Amorphous }^{\mathrm{c}} & \text { Amorphous } & \text { ZSM-5 } & \text { ZSM-48 } \\ \mathrm{Me}_{6} \text {-diquat-10 } & \text { Amorphous }^{\mathrm{c}} & \text { Amorphous } & \text { ZSM-48 } & \text { ZSM-48 }\end{array}$

${ }^{a} \mathrm{R}$ is the organic SDA and $\mathrm{y}$ is varied between $0 \leq \mathrm{y} \leq 1.0$,Crystallization was performed under rotation $(100 \mathrm{rpm})$ at $160{ }^{\circ} \mathrm{C}$ for 7 days, unless otherwise stated.

${ }^{\mathrm{b}}$ Final products are given in decreasing order of relative abundance based on powder XRD, and the phase obtained in a trace amount is given in parentheses. D and L are dense (mainly quartz) and layered phases, respectively

${ }^{\mathrm{c}}$ The material obtained after 14 days of heating at $160^{\circ} \mathrm{C}$.

\section{Material characteristics}

XRD patterns of ZSM-22 (C = commercial), ZSM-23, EU-1 and ZSM-48 are given in Figure 45. The XRD pattern of the in house synthesized ZSM-22 was similar. In some cases trace amounts of crystobalite was observed with respect to the latter sample. SEM micrographs show a clear difference comparing the morphology of the in house ZSM-22 (H) sample and commercial ZSM-22 (C). In house synthesized crystals were thinner and typically longer than the rod type crystals of ZSM-22 (C). The size of the commercial available ZSM-23 crystals seemed to vary from batch to batch. In the case shown in Figure 45 the ZSM-23 crystals were comparable in size to ZSM-22 (C) while in paper I the batch had thinner crystals. The EU-1 crystals were shown as elliptical shape in the first micrographs, but high resolution SEM micrographs implied that the crystals are agglomerates of even smaller crystals. Also for ZSM-48 small crystals were observed.

Both commercial samples, ZSM-23 and ZSM-22 (C), had a low BET surface area slightly below $200 \mathrm{~m}^{2} / \mathrm{g}$ as shown in Table 21 . The in house synthesized ZSM-22 gave a similar low surface area, but in the case of in house synthesized ZSM-23 (see experimental section for recipe) a drastic increase in surface area was observed. The material is now being tested in our group and show remarkably better catalytic stability compared to the commercial ZSM-23 catalyst. Slightly lower Si/Al ratios were found in the crystals compared to the synthesis mixture. One exception was ZSM-48 where the crystals had slightly higher $\mathrm{Si} / \mathrm{Al}$ ratio in the crystals compared to the synthesis mixture. 

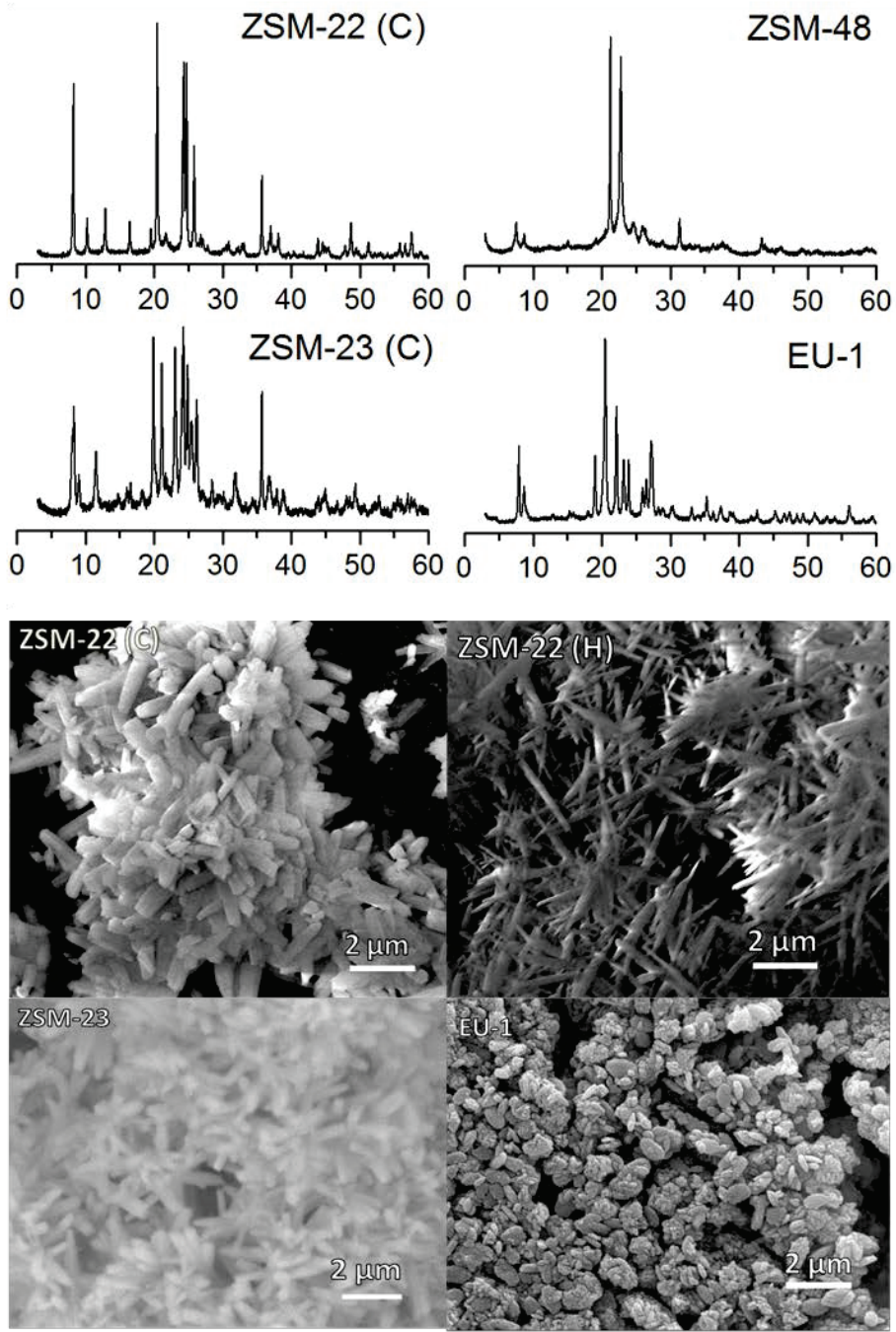

Figure 45: XRD patterns of ZSM-23, ZSM-22, EU-1 and ZSM-48 and SEM micrographs (see paper I for Pawley refinement and SEM micrographs of ZSM-48.

Table 21: Representative acid site density and surface area.

\begin{tabular}{|c|c|c|c|c|c|c|}
\hline Structure & $\begin{array}{c}\mathrm{Si} / \mathrm{Al}_{\mathrm{Ge}} \\
1\end{array}$ & $\begin{array}{c}\mathrm{Si} / \mathrm{Al}_{\mathrm{EDS}} \\
\text { (Crystals) }\end{array}$ & $\mathrm{Si} / \mathrm{Al}_{\text {Supplier }}$ & $\mathrm{Si} / \mathrm{Al}_{\mathrm{TPD}}$ & Crystals & $\begin{array}{c}\text { Surface area } \\
{\left[\mathrm{m}^{2} / \mathrm{g}\right]}\end{array}$ \\
\hline ZSM-23 (Zeolyst) & - & - & 33 & 26 & Needles $<1 \mu \mathrm{m}$ & 115 \\
\hline ZSM-23 (In house) & 30 & - & - & - & $\begin{array}{c}\text { Likely needles }< \\
0.5 \mu \mathrm{m}\end{array}$ & 299 \\
\hline EU-1 & 30 & - & - & 30 & Rods $<1 \mu \mathrm{m}$ & 420 \\
\hline ZSM-48 & 40 & - & - & 52 & Rods $1-2 \mu \mathrm{m}$ & 275 \\
\hline ZSM-22 (Zeolyst) & - & - & 50 & 48 & Rods $1-2 \mu \mathrm{m}$ & 196 \\
\hline ZSM-22 (In house) & 45 & - & - & 33 & Needles $2-3 \mu \mathrm{m}$ & 173 \\
\hline
\end{tabular}




\subsubsection{ZSM-57}

\section{Synthesis aspects}

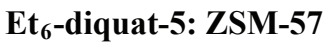

Instead of refluxing trimethylamines with dibromo chains it is possible to use ethylamines or propylamines etc. In the extensive study by Lee et al. [128] the influence of gel compositions on $\mathrm{Et}_{6}$-diquat-n SDAs were presented. In the case of the following gel composition: 3.0R: $9 \mathrm{Na}_{2} \mathrm{O}: \mathrm{yAl}_{2} \mathrm{O}_{3}: 30 \mathrm{SiO}_{2}: 1200 \mathrm{H}_{2} \mathrm{O}$ varying the amount of $\mathrm{Al}$, ZSM57 appeared when the $\mathrm{Si} / \mathrm{Al}$ ratio was 30 in the synthesis mixture. Similar to the $\mathrm{Me}_{6}$ diquat-n the SDA had low structure directing effect. This is not surprising since the bond connecting the $\mathrm{N}$ atom with the first $\mathrm{C}$ atom in the interconnecting chain is a single bond and hence allows rotation, combined with a flexible interconnecting chain and a risk of template decomposition in alkaline media.

Table 22: Table reprinted from [128].

\begin{tabular}{|c|c|c|c|c|c|}
\hline \multirow[t]{2}{*}{ Organic SDA } & \multicolumn{5}{|c|}{$\begin{array}{l}\text { 3.0R: } 9 \mathrm{Na}_{2} \mathrm{O}: \mathrm{yAl}_{2} \mathrm{O}_{3}: 30 \mathrm{SiO}_{2}: 1200 \mathrm{H}_{2} \mathrm{O}^{\mathrm{a}, \mathrm{b}} \\
\mathrm{Si} / \mathrm{Al} \text { in the synthesis mixture }\end{array}$} \\
\hline & 15 & 30 & 60 & & $\infty$ \\
\hline $\mathrm{Et}_{6}$-diquat-3 & ZSM-5 & ZSM-5 & Quartz + ZSM-5 & Quartz & \\
\hline $\mathrm{Et}_{6}$-diquat-4 & Mordenite & Mordenite & Quartz & Quartz & \\
\hline $\mathrm{Et}_{6}$-diquat-5 & Mordenite & ZSM-57 & $\mathrm{L}_{1}$ & $\mathrm{~L}_{1}$ & \\
\hline $\mathrm{Et}_{6}$-diquat- 6 & Mordenite & Quartz+Mordenite & Quartz & Quartz & \\
\hline $\mathrm{Et}_{6}$-diquat-7 & Mordenite & Mordenite & Quartz+Mordenite & $\mathrm{L}_{2}$ & \\
\hline $\mathrm{Et}_{6}$-diquat-8 & Mordenite & ZSM-5 & ZSM-5 & Quartz & \\
\hline $\mathrm{Et}_{6}$-diquat-9 & Mordenite & Quartz + ZSM-5 & Quartz + ZSM-5 & $\mathrm{L}_{2}$ & \\
\hline $\mathrm{Et}_{6}$-diquat-10 & Mordenite & ZSM-5+Mordenite & ZSM-5 & Quartz & \\
\hline
\end{tabular}

${ }^{a} \mathrm{R}$ is the organic SDA and $\mathrm{y}$ is varied between $0 \leq \mathrm{y} \leq 1.0$,Crystallization was performed under rotation $(100 \mathrm{rpm})$ at $160^{\circ} \mathrm{C}$ for 7 days.

${ }^{b}$ Final products are given in decreasing order of relative abundance based on powder XRD. Both $\mathrm{L}_{1}$ and $\mathrm{L}_{2}$ are known but probably layered phases.

\section{Material characterstics}

The XRD pattern and SEM micrograph of ZSM-57 is shown in Figure 46. The procedure resulted in plate like crystals with width $<2 \mu \mathrm{m}$ and thickness of a few tens of nanometres. TEM analysis revealed that the 10-ring was directing down the shortest crystal dimension and the 8-ring along the plate (will be published in the paper "Morphology induced shape selectivity"). 

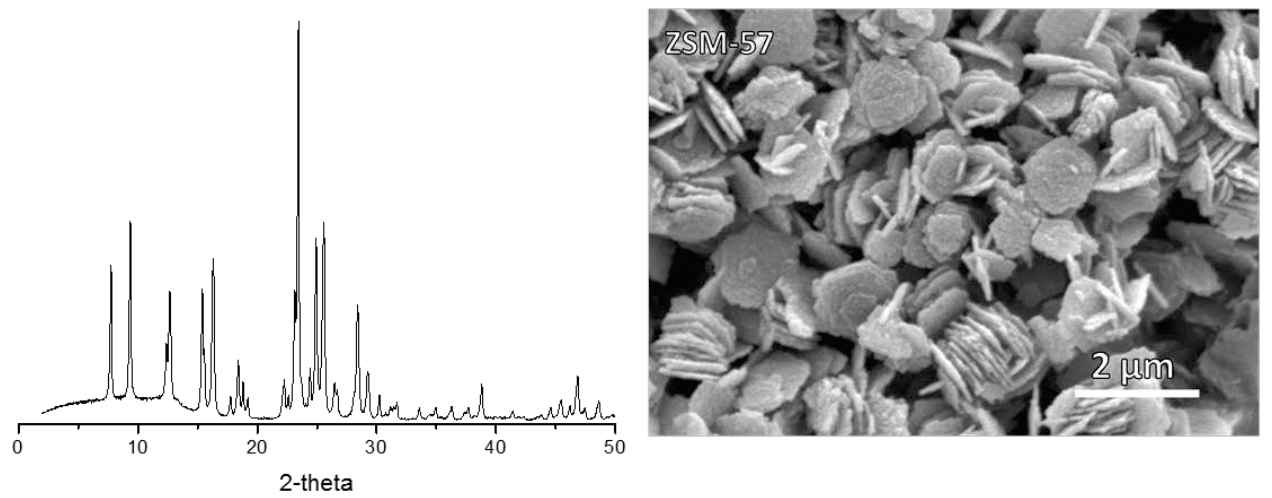

Figure 46: XRD patterns and SEM micrographs of ZSM-57 synthesized by the method described in the experimental section.

EDS analysis of the crystals gave a Si/Al ratio of 22, slightly lower than in the synthesis mixture and $\mathrm{N}_{2}$ physisorption measurement gave a specific surface area of $425 \mathrm{~m}^{2} / \mathrm{g}$ as shown in Table 23.

Table 23: Representative acid site density and surface area.

\begin{tabular}{cccccc}
\hline Structure & $\mathrm{Si} / \mathrm{Al}_{\mathrm{Gel}}$ & $\begin{array}{c}\mathrm{Si} / \mathrm{Al}_{\mathrm{EDS}} \\
(\text { Crystals })\end{array}$ & $\mathrm{Si} / \mathrm{Al}_{\mathrm{TPD}}$ & Crystals & $\begin{array}{c}\text { Surface area } \\
{\left[\mathrm{m}^{2} / \mathrm{g}\right]}\end{array}$ \\
\hline ZSM-57 & 30 & 22 & - & Plates $<2 \mu \mathrm{m}$ & 425 \\
\hline
\end{tabular}

\subsubsection{ITQ-13}

\section{Synthesis aspects}

\section{$\mathrm{Me}_{6}$-diquat-6: From $\mathrm{Br}$ to $\mathrm{OH}$}

Until now the SDAs presented have been in the bromide form. Further flexibility is possible when these SDAs are ion exchanged to obtain the $\mathrm{OH}$-form by ion exchange with resins. As described in the experimental section ITQ-13 is synthesized in highly concentrated gels by removing $\mathrm{NaOH}$ totally from the synthesis mixture. Instead $\mathrm{pH}$ was increased by the SDA itself (hexamethonium hydroxide - $\mathrm{HM}(\mathrm{OH})_{2}$ in the case of ITQ13) and charge balance was achieved by adding HF. In [19] the effect of Ge and $\mathrm{F}^{-}$was clearly established. On one side a much higher acid site density was obtained if Ge were 
added to the synthesis mixture. On the other side the competing phase of EU-1 was avoided when increasing the $\mathrm{F}^{-}$amount from one $\mathrm{F}^{-}$for each D4R. Similar amount of $\mathrm{F}^{-}$ and D4R yielded EU-1, but higher $\mathrm{F}^{-}$amounts yielded ITQ-13. A reduction of synthesis time was also evident after adding $\mathrm{Ge}$ and $\mathrm{F}^{-}$. In our case we experienced problems reproducing ITQ-13 with higher Al loadings at the given molar ratios. However slight increase in the amount of HF ( 0.56 instead of 0.50 - this may likely be increased further and give further increase in the incorporated amount of Al) yielded ITQ-13 and addition of seeds eliminated eventual problems of reproduction.

\section{Material characterstics}

The XRD pattern and SEM micrograph are given in Figure 47. In cases where the HF amount was kept at $0.5\left(0.910 \mathrm{SiO}_{2}: 0.010 \mathrm{Al}_{2} \mathrm{O}_{3}: 0.090 \mathrm{GeO}_{2}: \mathrm{xHF}: 0.250 \mathrm{HM}(\mathrm{OH})_{2}\right.$ : $5 \mathrm{H}_{2} \mathrm{O}$ ) the crystals were plate-like while slight increase to $0.56 \mathrm{HF}$ the crystals were needle-rise like. Another clear difference between the two samples was the amount of incorporated Al. The plate-like crystals had low Al amount and also low Ge amount as shown in Table 24 while a higher Al amount was detected in the case of needle shaped crystals. Based on these results a further increase in the HF amount could likely improve the Al incorporation in ITQ-13. The reasons why the herein obtained results differ slightly from [19] is not known, but it could be a result of batch size differences or stirring effects. 

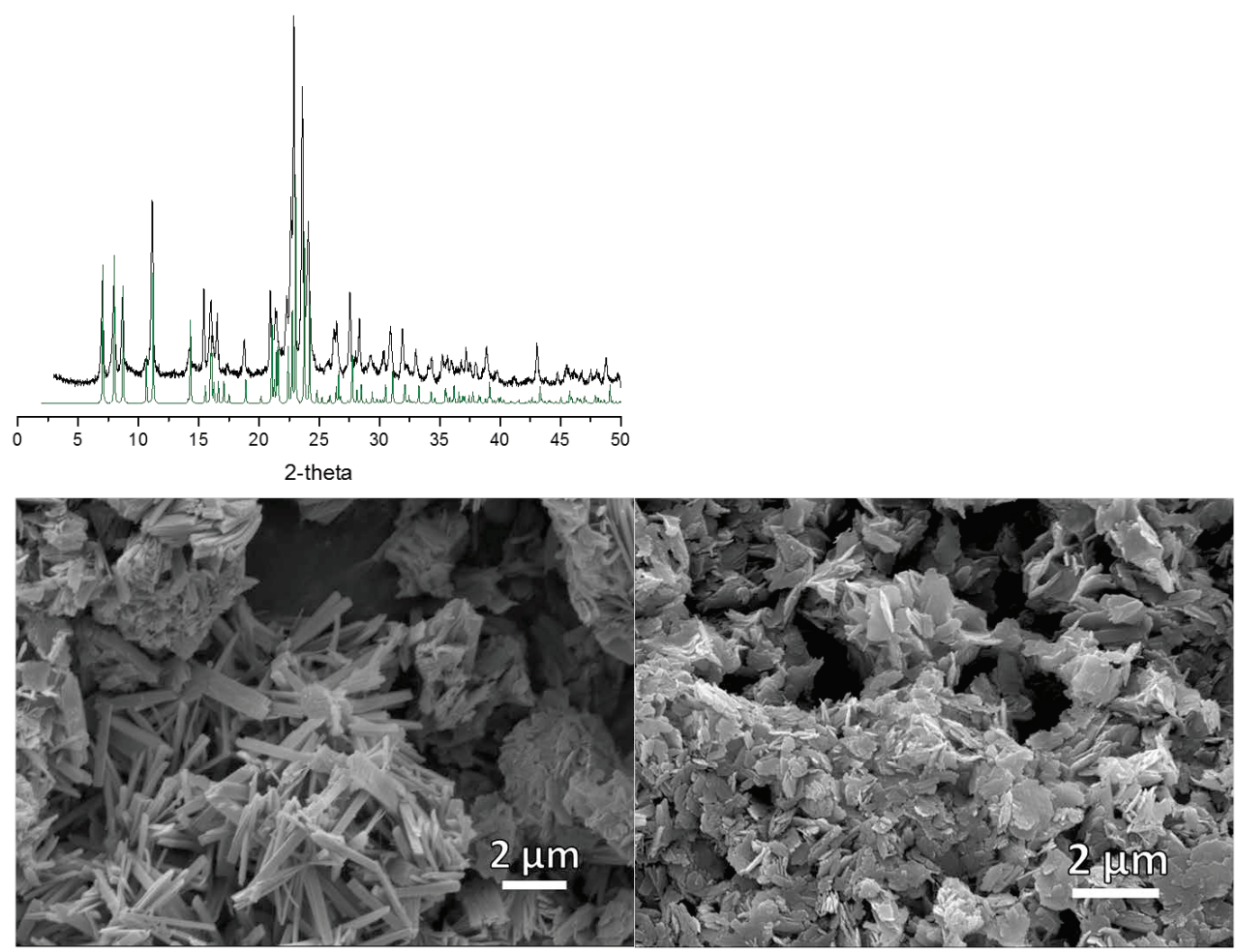

Figure 47: XRD and SEM micrographs. The synthesis resulted in needle-rise like particles in cases with increased HF amount while at lower amount of HF the crystals were plate-like.

Table 24: Representative acid site density and surface area.

\begin{tabular}{ccccc}
\hline Structure & $(\mathrm{Si}+\mathrm{Ge}) / \mathrm{Al}_{\mathrm{Gel}}$ & $(\mathrm{Si}+\mathrm{Ge}) / \mathrm{Al}_{\mathrm{EDS}(\text { Crystals })}$ & Crystals & $\begin{array}{c}\text { Surface area } \\
{\left[\mathrm{m}^{2} / \mathrm{g}\right]}\end{array}$ \\
\hline ITQ-13 $(\mathrm{N})$ & 50 & $(36.91+1.49) / 0.92 \sim 42$ & Needles & 413 \\
ITQ-13 $(\mathrm{P})^{\mathrm{a}}$ & 50 & $>100$ & Plates & 455 \\
\hline
\end{tabular}

${ }^{\text {a }}$ Less HF added to the gel compared to ITQ-13 (N) (reduced the amount of Ge and Al in the crystals).

Further description is found in paper II.

\subsection{Shape selectivity}

Further flexibility in the MTH product spectrum is important to meet fluctuations in the chemical market. Among such specific goals, reducing the amount of aromatics in the 
gasoline is an important task because of the increasing environmental constraints of the gasoline composition. Although aromatics are good octane boosters they may be carcinogenic. The commercially applied catalyst ZSM-5 typically yielded gasoline rich in such aromatics, but due to simple seeding synthesis systems, extremely long life times and products with high octane numbers, alternative zeolite catalysts that can compete with ZSM-5 on an economical base are hard to find. In this respect reducing the aromatic contribution in the gasoline is only one example of improved selectivity. Emphasis to find other zeolites with improved or different shape selective properties showing slow deactivation may lead to commercialization of products not commercialized today. In the present section a presentation of selectivities obtained from 1D, 2D and 3D zeolites will be given to illustrate our work on the way to find other product spectrums than the archetype ZSM-5 catalyst.

\section{D 10-ring zeolites}

In 2009 Teketel et al. [111] presented a highly interesting product spectrum of non aromatic $\mathrm{C}_{5}+$ (gasoline range) alkene fraction when methanol was reacted over the 1D 10ring ZSM-22 zeolite. It was stated that the zeolite could be used in the production of cleaner gasoline, but rather low feed rates (WHSV $=2 \mathrm{~h}^{-1}$ ) and high temperatures (400$500^{\circ} \mathrm{C}$ ) was required to obtain appreciable conversions. Interestingly, the result indicated that by choosing a catalyst topology with narrow pores the space demanding aromatic side of the dual cycle mechanism could be suppressed and hence favour the alkene driven side, yielding a higher concentration of $\mathrm{C}_{3}+$ hydrocarbons on the cost of $\mathrm{C}_{2}$, alkanes and aromatics. Based on the interesting selectivity obtained over ZSM-22 the study was extended (PAPER I) to other 1D 10-ring zeolites with slightly different channel size and different side pockets; ZSM-22, ZSM-23, EU-1 and ZSM-48 (see structural description in section 2.3.1 and synthesis procedures in section 4.1). In this way it could be possible to investigate how small differences in channel size influence the product spectrum and eventually if these zeolites could provide lifetimes exceeding the results obtained from ZSM-22.

The four 1D 10-ring zeolites were tested at $400^{\circ} \mathrm{C}$ and WHSV $=2 \mathrm{~h}^{-1}$ with resulting effluent compounds as shown in Figure 48. 

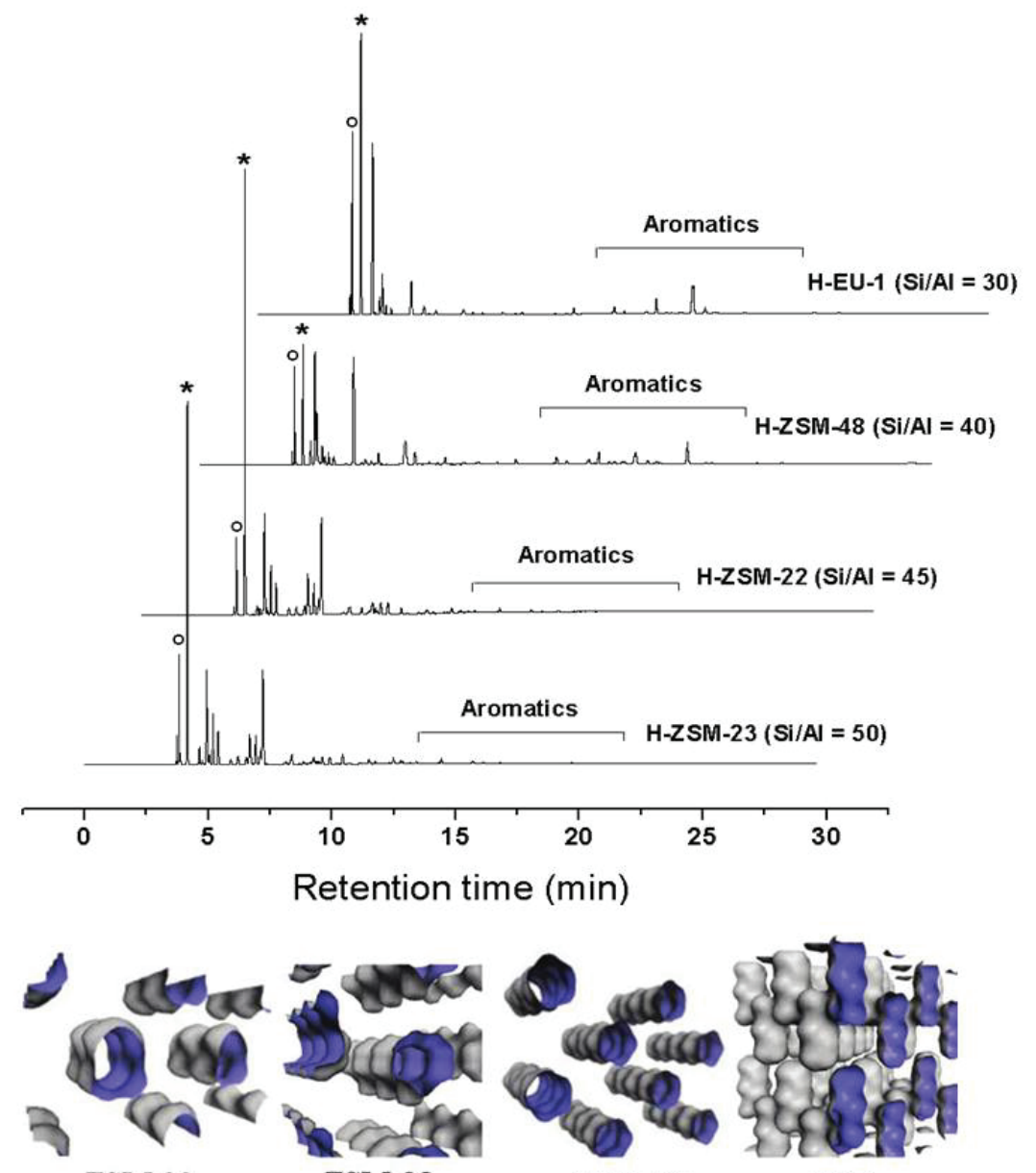

ZSM-22

ZSM-23

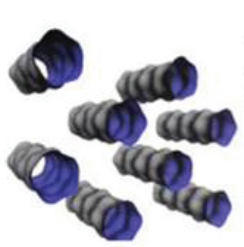

ZSM-48

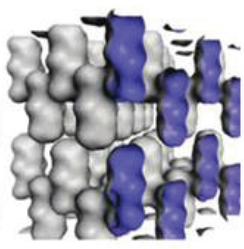

EU-1

Figure 48: Four $1 D$ zeolites, ZSM-22, ZSM-23, ZSM-48 and EU-1 tested in MTH. The corresponding effluent product spectrum obtained at $400^{\circ} \mathrm{C}$ and $W H S V=2 h^{-1}$ is given in the top right panel. The conversion is nearly full for ZSM-22, ZSM-23 and EU-1 while ZSM-48 had an initial conversion of $88 \%$. Figures adopted from [35].

All four zeolites were found to be active catalysts converting methanol to hydrocarbons. The catalysts gave initially nearly full conversion, except of ZSM-48 which initially had $88 \%$ conversion. Despite the similarities in channel dimensions there was a significant difference observed in the product spectrum. Very high selectivities towards $\mathrm{C}_{5}+$ hydrocarbons were found for ZSM-22 ( 50-70\%), ZSM-23 ( 40-70\%) and ZSM-48 ( 55-75 \%), but aromatic free fractions were found only over ZSM-22 and ZSM23 (20-40\% selectivity for aromatics in the $\mathrm{C}_{5}+$ fraction of ZSM-48). Despite the small 
channel size of EU-1 the product spectrum contained $\sim 15 \%$ aromatics in the $\mathrm{C}_{5}+$ fraction $(35-45 \%)$.

The higher aromatic yield in ZSM-48 may be ascribed to larger channels compared to ZSM-22 and ZSM-23 forming literarily a neglible concentration of aromatics. In situations where the dimensions of the pores are very similar to the size of the involved molecules, even small differences may lead to clearly affected diffusion rates and reactivity. $[69,129]$ Such seemed also to be the case for ZSM-48. The significantly higher aromatic fraction in EU-1 was surprising taking into account that the channel size is smaller than ZSM-22. However the aromatic contribution was in line with previous work on the same catalyst.[130] The reason for this is not clear, but the 12-ring side pockets are involved in the MTH reaction and might be exposed to the external surface which allows diffusion of aromatics out of the crystal. It can also be a result of changed retaintion time changing the obtained products. Overall, all catalysts tested involved aromatics, but it could diffuse out only in ZSM-48 and EU-1. This shows that even small differences may result in significantly changed product spectrum.

\section{ITQ-13 - a 3D zeolite structure with narrow channels}

The promising selectivity obtained over ZSM-22 and ZSM-23 led us to ask if also other zeolites with narrow pores could lead to an improved product spectrum with respect to gasoline or small alkene synthesis different from ZSM-5. In this perspective a zeolite, ITQ-13 (Description of the material is given in section 2.3.3) with many similarities to ZSM-5, but with narrower pores was synthesised in highly concentrated Ge-containing gels with the fluoride route (section 4.1), PAPER II. The ITQ-13 sample ((Si+Ge)/Al ratio 42 with needle shaped crystals $<3 \mu \mathrm{m} \times 100 \mathrm{~nm}$ ) was tested in similar condition as ZSM-5 (Si/Al ratio 50 with crystals typically $<2.5 \mu \mathrm{m}$ ) and ZSM-22 (Si/Al ratio 50 with rod shaped crystals typically 1-3 $\mu \mathrm{m}$ in length) at $400^{\circ} \mathrm{C}$ and WHSV $=2 \mathrm{~h}^{-1}$ for comparison of the effluent selectivity. Detailed gas chromatograms of the effluent from ZSM-5, ZSM-22 and ITQ-13 at full methanol conversion at $400{ }^{\circ} \mathrm{C}$ are shown in Figure 49. Both ITQ-13 and ZSM-5 gave similar product cut-off with tetra methylbenzenes (tetraMB) as the heaviest significant product identified. Only traces of higher polyMBs were visible in the chromatogram. As discussed in Paper I ZSM-22 gave mainly linear and branched alkenes with neglible amount of aromatics. Surprisingly, despite that the 
channels in ITQ-13 are narrower than ZSM-22 more aromatics formed over ITQ-13. Causes for this have not been straightforward to identify since such molecules would be expected to be trapped in the pore system of this catalyst. The aromatic formation might be a result of pore breathing, exposed intersections or exposed side pockets as suggested in the case of EU-1.[35]

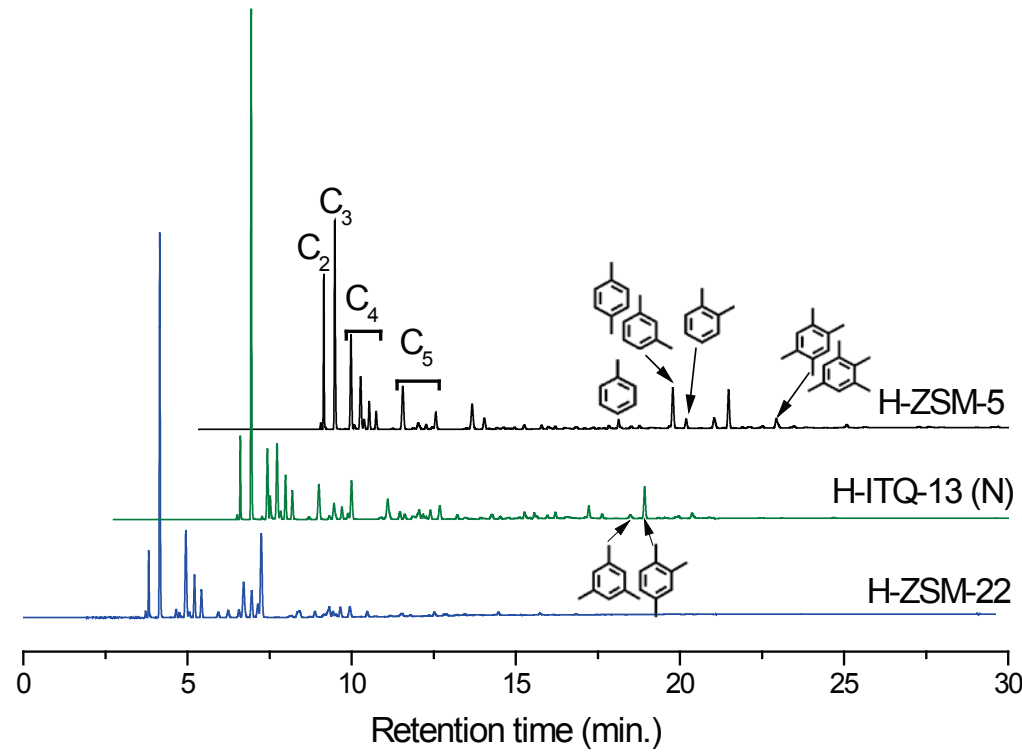

Figure 49: Chromatograms of the effluent composition for ZSM-22, ZSM-5 and $I T Q-13$ at $400^{\circ} \mathrm{C}$ and $W H S V=2 h^{-1}$.

A comparison of essential selectivity parameters for ITQ-13, ZSM-5 and ZSM-22 as a function of methanol conversion at $400{ }^{\circ} \mathrm{C}$ (Figure 50) showed that ITQ-13 gave higher propene selectivities than ZSM-5 and ZSM-22 at the highest conversion and could be a potential material for the Methanol to Propene (MTP) process. As the conversion dropped ITQ-13 gave slightly lower propene selectivities and slightly higher $\mathrm{C}_{5+}$ selectivities than ZSM-5, whereas ZSM-22 gave much higher $\mathrm{C}_{5+}$ selectivities, and lower $\mathrm{C}_{3}$ selectivities, than both ITQ-13 and ZSM-5. The hydrogen transfer index (HTI) defined as the selectivity toward butanes divided on the total $\mathrm{C}_{4}$ selectivity (Figure 50, left panel), showed that ITQ-13 was closer to ZSM-5 than to ZSM-22. In accordance with the dualcycle the MTH reaction is assumed to be a sequential reaction where light alkenes first form and may undergo methylation to form higher alkenes and thereafter form aromatics 
and alkanes by intermolecular hydrogen transfer. The structures that had low ability to form aromatic products (ZSM-22 and ZSM-23) in the effluent typically also showed a lower $\mathrm{C}_{4}$ HTI than compared with structures giving aromatics in the effluent. It appears that aromatization is spatially hindered in ZSM-22 while in both ITQ-13 and especially ZSM-5 (with more spacious channel intersections) aromatics form. Hence the $\mathrm{C}_{4}$ HTI of ITQ-13 was in accordance with its effluent selectivity to aromatic products, which was also intermediate between the two (Figure 49).
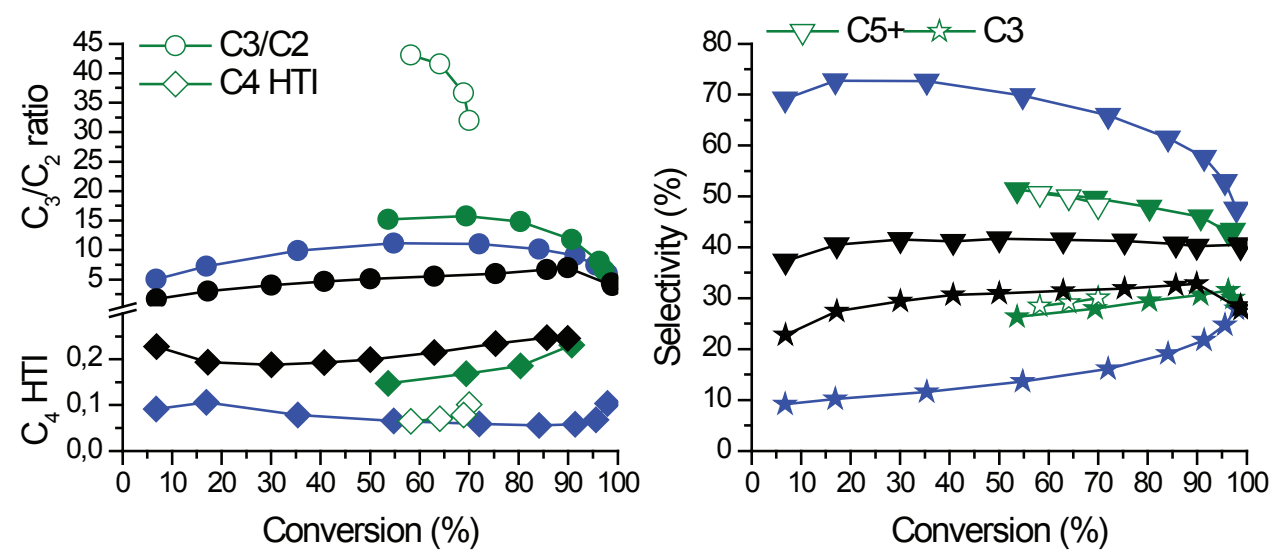

Figure 50: Comparison of critical parameters of ITQ-13, ZSM-22 and ZSM-5 at $400^{\circ} \mathrm{C}$ and WHSV $=2 h^{-1}$. The $C_{3} / C_{2}$ ratio and the $C_{4} H T I$ are shown in the left panel, while the selectivity towards $C_{3}$ and $C_{5}+$ is shown in the right panel. Blue symbols: ZSM-22, Closed green symbols: ITQ-13, black symbols: ZSM-5 (green open symbols is another ITQ-13 sample with Si/AI > 100 and plate-like morphology instead of needles). Because ZSM-5 showed very slow deactivation the WHSV was changed from 2 to $9 \mathrm{~h}^{-1}$ after $70 \mathrm{~h}$ on stream. Thus for ZSM-5 the first point at maximum conversion is measured at WHSV $=2 h^{-1}$ and the subsequent points at $9 h^{-1}$.

Another ITQ-13 batch, ITQ-13(P), which had a much lower acid site density compared to ITQ-13(N) and plate-like morphology instead of needles, resulted in a $\mathrm{C}_{4}$ HTI similar to ZSM-22. The $\mathrm{C}_{3}$ and $\mathrm{C}_{5}+$ fractions were rather similar for the two ITQ-13 batches. However the ITQ-13 (P) batch yielded a product spectrum with less ethene and less than half of the aromatics $(2.3 \%$ ) in the effluent (at $\sim 70 \%$ conversion) as compared to and $6 \%$ aromatics in ITQ-13 $(\mathrm{N})$. Unfortunately, despite the promising selectivity, the initial conversion of the ITQ-13 (P) sample was only $70 \%$, likely a result of the lower acid site density of this sample.

High pressure tests of ITQ-13 $(\mathrm{N})$ were carried out with a hypothesis that it would 
increase the gasoline fraction. At $30 \mathrm{bar}, 400^{\circ} \mathrm{C}$ and $\mathrm{WHSV}=2 \mathrm{~h}^{-1}$ the $\mathrm{C}_{5}+$ fraction clearly increased to $65-75 \%$ from $\sim 55 \%$ at ambient conditions as shown in Figure 51. Despite improvement in this fraction the aromatic fraction increased to $15-30 \%$ aromatics in the effluent selectivity. Additionally there was a ten-fold decrease in methanol conversion capacities at high pressure.

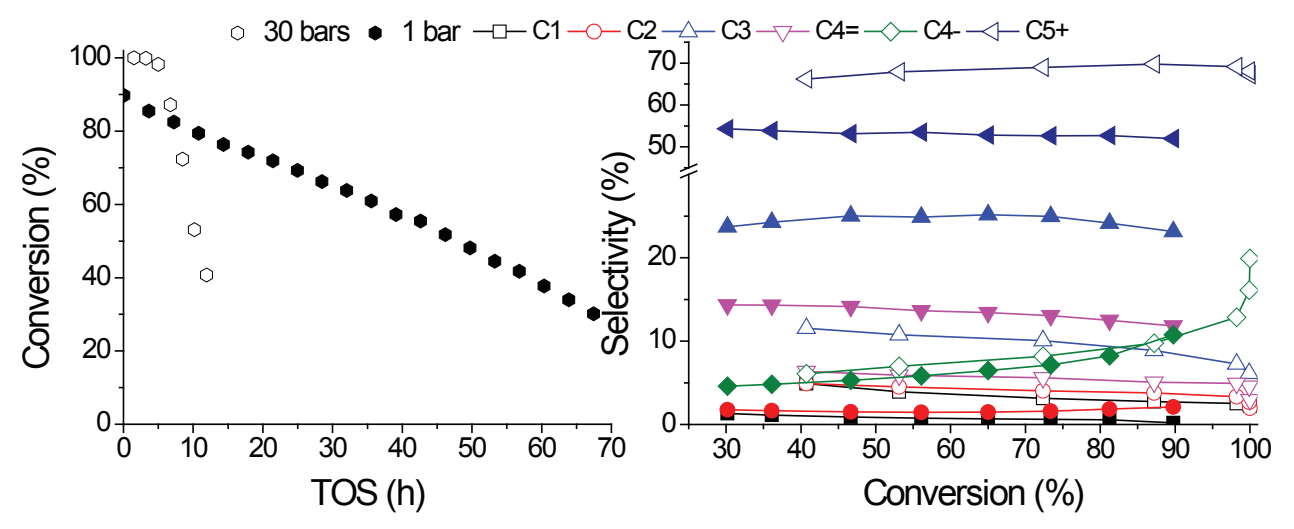

Figure 51: Left panel: Conversion versus TOS (h) at ambient (closed symbols) and high pressure (30 bar) (open symbols) at $350^{\circ} \mathrm{C}$ and $\mathrm{WHSV}=2 h^{-1}$. Right panel: Selectivity versus conversion for high pressure (open symbols) and ambient pressure (closed symbols).

In summary and to the best of our knowledge this was the first time ITQ-13 was tested as catalyst in the MTH process. Despite the formation of aromatics in the product effluent, especially at high pressures, the ITQ-13 structure gave a product spectrum intermediate of ZSM-22 and ZSM-5 under reaction conditions at ambient pressure and hence adds to the product spectrum library gaining further flexibility in methanol conversion to hydrocarbons over zeolite catalysts.

Still, a question arises - what influence do the intersections have on the product spectrum? According to the findings of 1D materials small differences gave clearly different selectivity (e.g. more aromatics formed over ZSM-48). If only counting the channel size ITQ-13 should have produced even smaller products than ZSM-22, but clearly this is not observed experimentally. Exposed intersection volumes or pore breathing effects may be a possible explanation to the observed aromatics in the effluent. 


\section{Conversion of methanol over 10-ring zeolites with differing volumes at channel intersections: comparison of TNU-9, IM-5, ZSM-11 and ZSM-5.}

Four 10-ring zeolites, IM-5, TNU-9, ZSM-11 and ZSM-5 were tested as catalyst in the MTH reaction at atmospheric pressure at $350^{\circ} \mathrm{C}$ and $\mathrm{WHSV}=9 \mathrm{~h}^{-1}$. IM-5 and TNU-9 were synthesized according to the recipes in 4.1. ZSM-11 and ZSM-5 were supplied from the laboratories of Haldor Topsøe and ZeoCat respectively. All catalyst had crystal sizes below $2 \mu \mathrm{m}$ and the $\mathrm{Si} / \mathrm{Al}$ ratio was in the range of 14-24. Table 25 shows that the materials have quasi-similar properties. The main difference between the catalysts was the differing volumes at channel intersections.

Table 25: Material properties.

\begin{tabular}{lcccc}
\hline & TNU-9 & IM-5 & ZSM-11 & ZSM-5 \\
\hline Si/Al (microprobe) & 14 & 13 & 24 & 17 \\
Si/Al (EDS) & 15 & $14-18$ & 22 & 17 \\
$\mathrm{Si} / \mathrm{Al}$ (TPD) & 17 & 16 & $*$ & 20 \\
$\begin{array}{l}\text { Crystal/agglomerate size } \\
\text { (microns) }\end{array}$ & $0.2-1$ & $0.2-1$ & $1-2$ & $0.2-2$ \\
$\begin{array}{l}\text { BET surface area }\left(\mathrm{m}^{2} / \mathrm{g}\right) \\
\text { BET micropore volume }\left(\mathrm{cm}^{3} / \mathrm{g}\right)\end{array}$ & 0.191 & 0.141 & 0.170 & 0.152 \\
\hline
\end{tabular}

In previous discussed papers of ZSM-5 both the aromatic and the alkene cycle were assumed to operate simultaneously in spacious channels with intersections. On the contrary the results obtained by Teketel et al. [110, 111] over ZSM-22 showed that aromatic part of the dual cycle could be hindered if the pore space is restricted (no intersection/less spacious channels) both results implied that controlling the pore space available controls the product spectrum. This conclusion led to the investigation of the $3 \mathrm{D}$ 10-rings with differing volumes at channel intersections to elucidate the effect of intersection size on the product selectivity (PAPER III). 
TNU-9 and IM-5 were tested for the first time as catalysts in MTH together with the well known ZSM-5 and ZSM-11 catalysts. All four materials were active catalyst under the reaction conditions mentioned above and gave initially full methanol conversion. The product effluent was strikingly similar as shown in Figure 52. However, detailed analysis of the yield versus conversion plots showed a shift towards more heavily methylated benzenes in TNU-9 and IM-5 (penta- and hexamethylbenzenes appear in the effluent as unstable products) than ZSM-11 and ZSM-5 (no sign of penta- and hexamethylbenzenes).

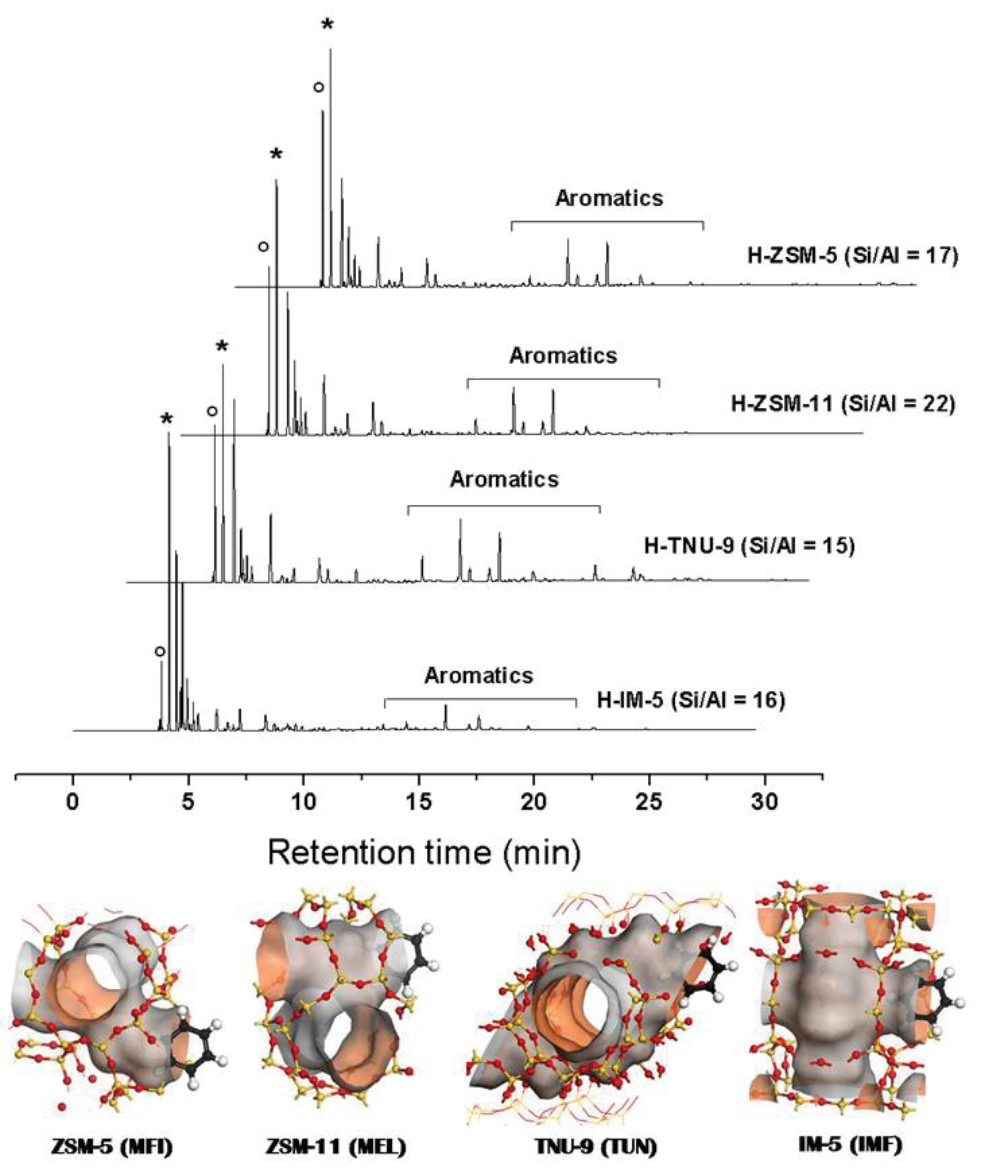

Figure 52: Four 3D zeolites, ZSM-5, ZSM-11, TNU-9 and IM-5 tested in MTH. The corresponding effluent product spectrum obtained at $350^{\circ} \mathrm{C}$ and $\mathrm{WHSV}=9 \mathrm{~h}^{-1}$ after 5 minutes on stream. All materials started at full methanol conversion. Figures show the 
largest channel intersection in the various structures. The channel intersections are adopted from [131].

Although the effluent was strikingly similar the retained species in the zeolites presented in Figure 53 were clearly different. In ZSM-5 and ZSM-11 only methylated benzenes were detected in the coke analysis while TNU-9 and IM-5 aromatics containing two, three and four benzene rings were among the entrapped species.

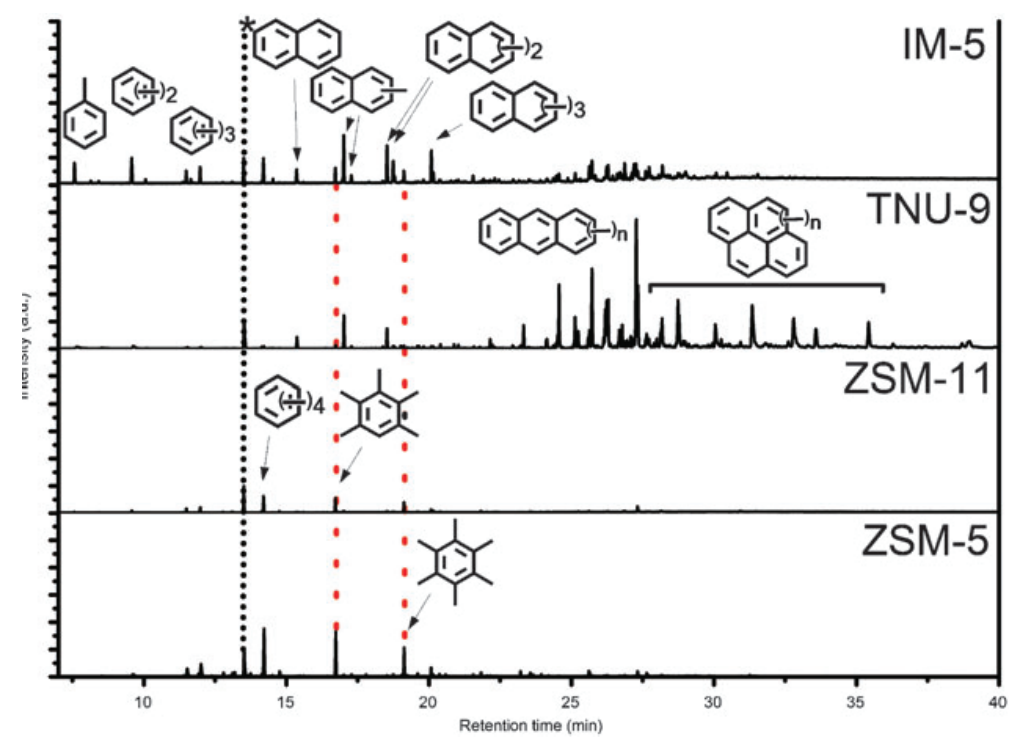

Figure 53: Chromatograms showing the retained species in ZSM-5, ZSM-11, TNU-9 and $I M-5$ after the end of the experiment $\left(350^{\circ} \mathrm{C}, W H S V=9 h^{-1}\right)$.

In summary the different intersection volumes did not result in any major differences in the gas effluent indicating that it is mainly the channel size and constraints from the channels that is of major importance (here we assume that the zeolites produces much more gas effluent products relative to the entrapped hydrocarbons).

\subsection{Deactivation}

A clear difference between the four 3D 10-ring catalysts was the deactivation rate. TNU-9 and IM-5 gave more rapid deactivation compared to ZSM-5 and ZSM-11 as shown in Figure 54 . While IM-5 dropped to $20 \%$ conversion after approximately 5 hours on stream ZSM-11 showed practically negligible deactivation until $\sim 30$ hours at similar conditions. 
This significant difference was ascribed to the very different compounds trapped in the zeolite during reaction. Even though TNU-9 and IM-5 had been much shorter on stream before coke dissolution the amount of dissolvable species was much higher. Despite that the Si/Al ratio of ZSM-5 (17) and ZSM-11 (22) was slightly higher (although in a narrow range) than TNU-9 (15) and IM-5 (16) the results implied that a link between the heavy entrapped species and deactivation rate exists.[131] Hence it seemed that the high selectivity to coke led to rapid deactivation.

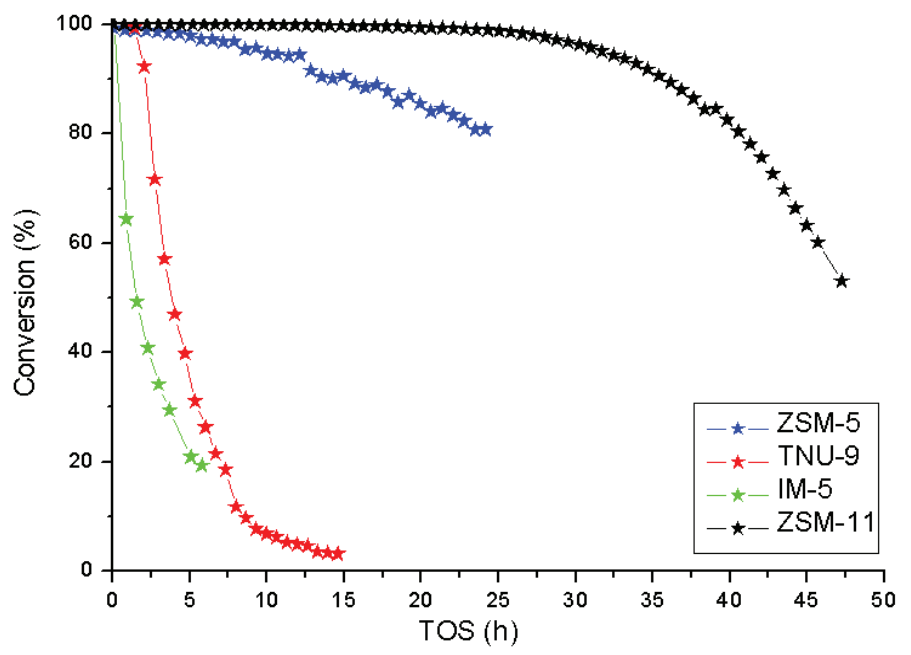

Figure 54: Conversion versus TOS curves obtained from ZSM-5, ZSM-11, TNU-9 and $I M-5$ at $350^{\circ} \mathrm{C}$ and $W H S V=9 h^{-1}$. TNU-9 and IM-5 gave more rapid deactivation compared to ZSM-5 and ZSM-11

\section{Investigating coke formation over zeolites in fluid bed reactors by In situ Raman spectroscopy}

The results from the four 3D 10-rings (PAPER III) lead to initial spectroscopic studies of TNU-9, IM-5 and ZSM-5 under reaction conditions (PAPER IV) with defined pulses of methanol towards the fluidized bed reactor described in 4.2.1. The aim was to gain insight into how coke develops and eventually which precursors or species that are involved in coke formation resulting in deactivation of the materials. It was expected that spectra obtained from ZSM-5 would give support to the slower deactivation observed for this material and give insight towards mechanism involved in coke formation over the herein tested zeolites.Figure 55 displays the Raman spectra of ZSM-5 (top panels), TNU-9 (mid 
panels) and IM-5 (down panels) (Ar flow at $350^{\circ} \mathrm{C}$ ). The activated zeolite (before the first methanol dose) is shown in black while colored graphs were collected after a distinct number of methanol pulses described in the figure. First concentrating on the zeolite spectra, the part of the spectrum ranging from $1000-250 \mathrm{~cm}^{-1}$ is the fingerprint of the investigated zeolite, involving Si-O vibrational modes and is not devoted to coke or other carbon species. The bands around at $800 \mathrm{~cm}^{-1}$ and at $1000-1200 \mathrm{~cm}^{-1}$ corresponds to symmetric and asymmetric stretching vibrations in the framework $(\mathrm{T}-\mathrm{O}-\mathrm{T}, \mathrm{T}=\mathrm{Si}$ or $\mathrm{Al})$ respectively.[132, 133] In addition to the framework vibrations the sharp band, marked with an (*) appears from $\mathrm{O}_{2}$ in air when using the $\lambda_{\text {exc }}=244 \mathrm{~nm}$ line and is also not devoted to hydrocarbons.

Concentrating on ZSM-5 in the upper panels in Figure 55, a detailed analysis of the Raman spectra showed no significant difference comparing the activated zeolite and the two first pulses of methanol. After 6 pulses a band rapidly grew with peak at $1607 \mathrm{~cm}^{-}$ ${ }^{1}$ (and evolved towards $1597 \mathrm{~cm}^{-1}$ after the prolonged pulse (green)) reveals the build up of hydrocarbons in the pore system of the catalyst. After 14 pulses a band around at 1367 $\mathrm{cm}^{-1}$ in addition to bands around at $1225 \mathrm{~cm}^{-1}$ had evolved. Hence two major bands, one around at $1600 \mathrm{~cm}^{-1}$ and another around at $1367 \mathrm{~cm}^{-1}$, evolved after a distinct number of methanol pulses. It should here be noted that after the long pulse and Ar flush the sample movement got slower due to technical issues. However after the long pulse broad bands around at $1400 \mathrm{~cm}^{-1}$ and $1200 \mathrm{~cm}^{-1}$ in addition to the intense band at $1597 \mathrm{~cm}^{-1}$ were visible. In previous Raman investigations on molecular and extended aromatic carbon species two intense bands at $1600 \mathrm{~cm}^{-1}$ (denoted the $\mathrm{G}$ band) and $1350 \mathrm{~cm}^{-1}$ (denoted the $\mathrm{D}$ band) have commonly been assigned to different $\mathrm{sp}^{2}$-bonded $\mathrm{C}$ atoms with various degree of graphitic ordering, ranging from microcrystalline graphite to glassy carbon.[134-137] Typically the G band has been connected to in plane bond stretching of pairs of hybridized carbon atoms in graphitic carbon. This is not restricted to six fold rings, but appears for many molecules having a $\mathrm{sp}^{2}$ center. Dependent on the degree of order of the $\mathrm{sp}^{2}$ center it could range from $1500-1690 \mathrm{~cm}^{-1}$. For graphitic ring systems the frequency is typically lower than $1600 \mathrm{~cm}^{-1}$, but for chain type $\mathrm{sp}^{2}$ carbons short or strained $\mathrm{C}=\mathrm{C}$ bonds can reach frequencies up to $1690 \mathrm{~cm}^{-1}$. The D band appears when analyzing disordered graphite-like species as it is forbidden in perfect graphite. It is strictly connected to the presence of six fold aromatic rings although the intensity varies 
with photon excitation energy. Since this band was shown to be neglible, both in amorphous and graphitic carbon, when using a laser line in the UV range $(\lambda=244 \mathrm{~nm})$, it was concluded that the $\mathrm{D}$ band was due to superimposition of the vibrational modes of aromatic ring molecules rather than larger extended structures. [134, 135] As also described in [121] the use of $G$ and $D$ term is not sufficient for the description of the spectra at this stage. 


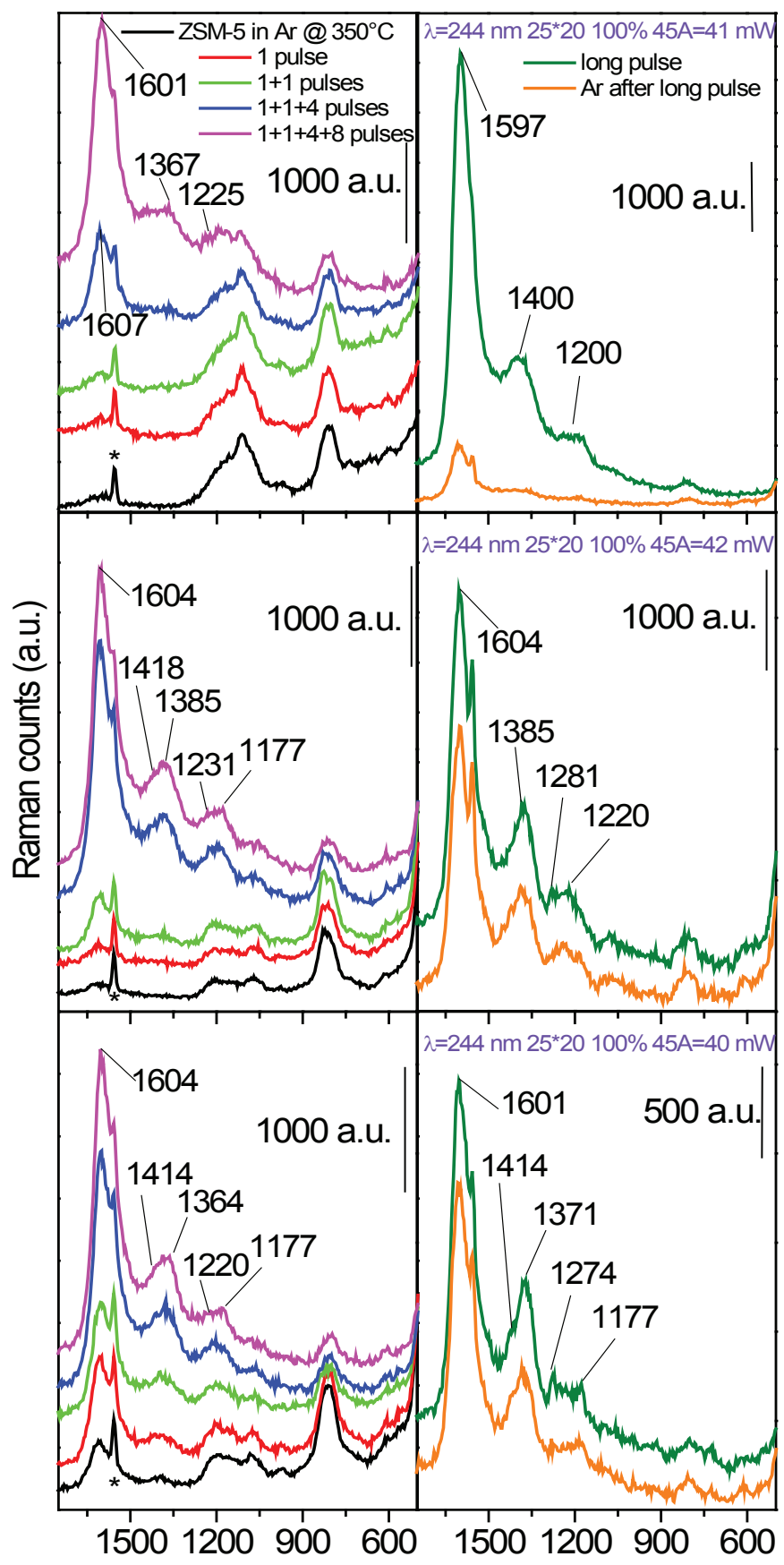

Figure 55: Raman spectra of activated zeolite (black curve) and after a certain number of methanol pulses (colored). ZSM-5 is shown in the top panels, TNU-9 in the mid panels 
and IM-5 in the bottom panels. At the end of each experiment a prolonged pulse was given. It should be noted that we experienced technical problems resulting in slow sample movement with respect to the long pulse for TNU-9 and ZSM-5 and after the long pulse flushing with Ar for the ZSM-5 sample. Hence the experiments from the particular materials will be repeated.

Comparing the spectra of ZSM-5 with TNU-9 (mid panels) one major difference can be seen already from the second methanol pulse as the $G$ band immediately grew for the latter sample. Another clear difference was that even after only 6 methanol pulses also the $\mathrm{G}$ band had clearly evolved together with bands around at $1200 \mathrm{~cm}^{-1}$ (broad). Evaluating the long pulse of TNU-9 the initial growth of bands around at $1200 \mathrm{~cm}^{-1}$ had developed with center of the band moving towards higher Raman shifts (14 pulses: between 1177$1231 \mathrm{~cm}^{-1}$, after a long pulse: between $1220-1281 \mathrm{~cm}^{-1}$ ). The more rapid development of distinct bands, filling up the internal pore system of this catalyst, correlate well with the low observed catalytic stability of TNU-9 in comparison with ZSM-5. There might be indications of a small shoulder at $1418 \mathrm{~cm}^{-1}$ developed after 14 methanol pulses. Several polycondensated aromatics (e.g. phenanthrene, anthracene) show Raman lines in this region. Such species should not be able to leave the pores of this zeolite unless the large intersection volumes are exposed to the terminating side of the crystal. Still after Ar flush the shoulder seemed to be present and interestingly the band around at $1600 \mathrm{~cm}^{-1}$ was very less affected by inert gas flow which support that these species were stuck in the catalyst in line with [121].

Adding the spectrum of IM-5 to the results the activated sample had a slighty developed $\mathrm{G}$ band already before methanol onset, probably due to stable residues from calcination of the SDA. However the rapid development of bands observed was in line with TNU-9 and the results from the MTH tests performed in [131]. There was a tendency of slightly more resolved bands at $1177 \mathrm{~cm}^{-1}$ and $1274 \mathrm{~cm}^{-1}$ (after long pulse) based on the spectra obtained from IM-5, the former band was also visible for the two other structures around similar Raman shifts. The shoulder at the D band at approximately $1414 \mathrm{~cm}^{-1}$ (see after long pulse and after Ar flush) showed that also for this structures polycondensated species were likely present in line with the spectra obtained from TNU9.

The overall results implied that polycondensed species to a lower extent form in ZSM-5 compared to the other two materials with larger intersection volumes in line with Figure 53. Further, the results clearly showed that coke development was more rapid in 
TNU-9 and IM-5 compared with similar doses of methanol over ZSM-5. These results correlate well with the size and the intersection volumes, assumed to cause more rapid deactivation because of coke formation. Also transferring the results from the $G$ and the $D$ band to the present study, the single initial growth of the $\mathrm{G}$ band may indicate that noncyclic alkenes (e.g. butadienes, pentadienes) form in the early stage of the reaction. The fact that the D band, evolving from superimposition of the vibrational modes of species with aromatic rings, grew at a later stage than the $G$ band, may support that over time these non cyclic alkenes turn into cyclic (aromatic like) compounds.

\subsection{Improvements in lifetime}

The materials exposed to alkaline treatment or a combined micture of $\mathrm{NaOH}$ and surfactant $(\mathrm{CTAB})$ produced a substantial amount of data. The main results are therefore highlighted in Table 26 before a more detailed description of the results is given below.

Table 26: Main findings from alkaline treated materials and materials exposed to combined mixtures of $\mathrm{NaOH}$ and surfactant.

\begin{tabular}{lcccc}
\hline Structure & \multicolumn{2}{c}{ Alkaline treatment } & $\begin{array}{c}\text { Combined alkaline and surfactant } \\
\text { treatment }\end{array}$ \\
\cline { 2 - 5 } & Mesopores & $\begin{array}{c}\text { Improved catalytic } \\
\text { performance }\end{array}$ & Mesopores & $\begin{array}{c}\text { Improved catlytic } \\
\text { performance }\end{array}$ \\
\hline ZSM-22 & Yes & No & Yes & No \\
ZSM-23 & No & No & No & No \\
ZSM-57 & Minor & No & Yes & No \\
TNU-9 & Yes & No & Yes & Yes \\
IM-5 & Yes & Yes & Yes & Yes \\
ITQ-13 & No & Yes & Yes & Yes \\
ZSM-5 & Yes & No & Likely & No \\
\hline
\end{tabular}

*Started at lower conversion, but deactivate slower than the parent material. 


\section{Morphology is critical for the desilication}

process - ZSM-22

Even though the product spectrum of ZSM-

22 was the most promising among the selectivities obtained here, the rapid deactivation even at low feed rates remained an issue. Promising results from desilication procedures on other zeolites initiated such studies over ZSM-22 with the aim of reducing diffusion limitations. In house synthesized ZSM-22 was synthesized according to the recipe given in section 4.1

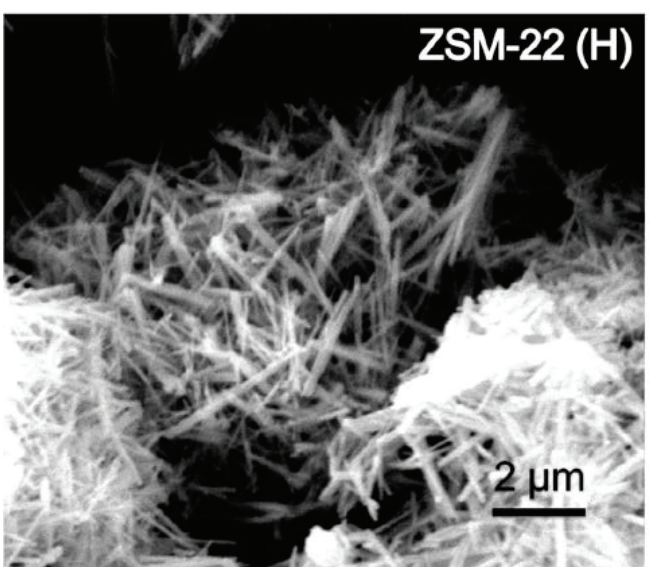

Figure 56: SEM micrographs of in house synthesised ZSM-22 and had needle shaped crystals as shown in Figure 56. The samples were exposed to solutions with concentrations of $0.05 \mathrm{M}, 0.25 \mathrm{M}$ and $0.5 \mathrm{M} \mathrm{NaOH}$ of $\mathrm{NaOH}$ for $0.5 \mathrm{~h}$ in water baths. $\mathrm{N}_{2}$ sorption measurements showed no sign of a hysteresis curve and no mesopores were introduced. Attempts by using the Carbon Black method described in section 2.5.4 were also not successful. Synthesis of ZSM-5 was possible, but the wrong phase was obtained for all attempts to synthesize mesoporous ZSM-22. Because of these difficulties the Sugar method described in section 2.5.3, was applied instead. In contrast to the Carbon Black method the right phase could be obtained, even by using a silica source several time impregnated with sugar with subsequent pyrolysis after each impregnation crystalline ZSM-22 was visible. The $\mathrm{N}_{2}$ sorption isotherms (Figure 57) showed to our disappointment no sign of hysteresis loop and hence no mesopores were present. Also TNU-9 could be obtained by this method, but as was the case of ZSM-22 no mesopores were visible. As a consequence these materials were never tested as catalysts in MTH. 

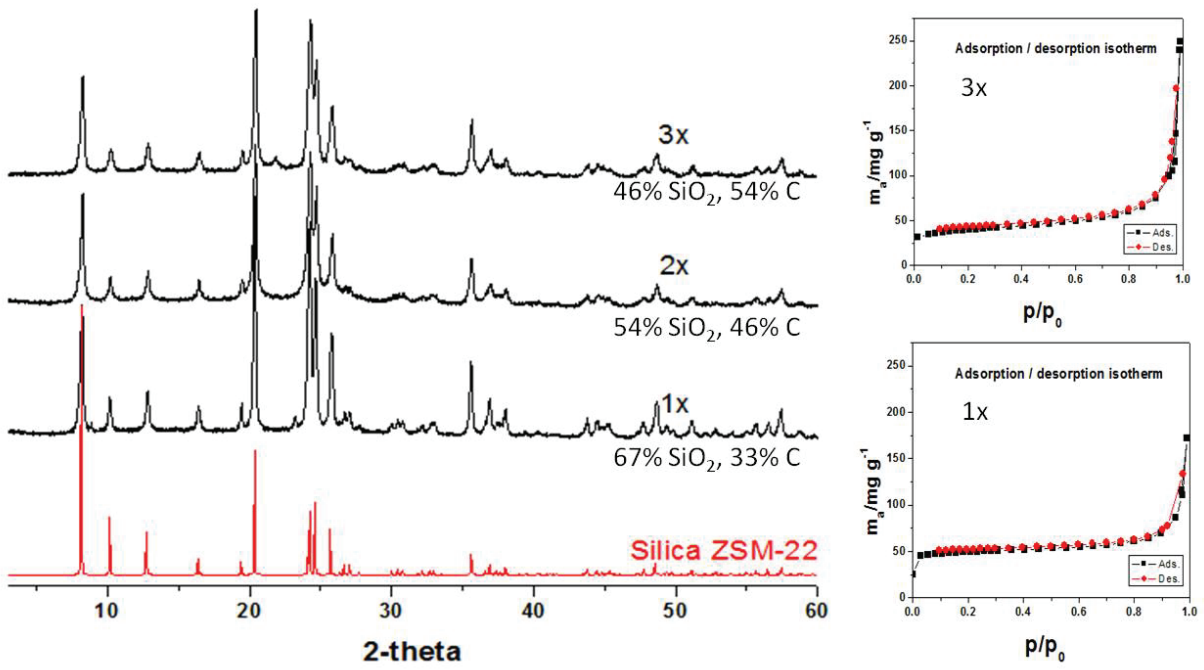

Figure 57: The crystallinity was kept after several impregnations with sugar with subsequent pyrolysis in inert atmosphere. After calcination the sorption measurement showed no mesopore formation.

The difficulties of introducing mesopores in ZSM-22 led to the speculation that the morphology (thin and long needles) was not optimal for mesopore introduction by desilication or carbon templating since the crystals would probably break instead of resulting in a mesopore because the mesopore itself is not much smaller than the thin crystal dimension. Also, thinner crystals would possibly also lead to many Al surface atoms hindering mesopore formation. Hence a new batch with a morphology different from the in house synthesized sample (thicker rods, $\mathrm{Si} / \mathrm{Al}=50$ ), was exposed to different $\mathrm{NaOH}$ solutions $(0.2 \mathrm{M}-1.0 \mathrm{M})$ for various times $(0.5-1.5 \mathrm{~h})$. More pronounced hysteresis loops, shifted to higher partial pressures, upon harsher treatment, indicated mesoporosity as shown in Figure 58 left panel. The BJH plot (right panel) indicated increased pore size supported by a clear increase in the mesopore volume, but the micropore volume dropped after treatment. In contrast to the initial ZSM-22 batch with thin needle shaped crystals thicker rods resulted in mesopores, suggesting a clear correlation between morphology and mesopore incorporation. 

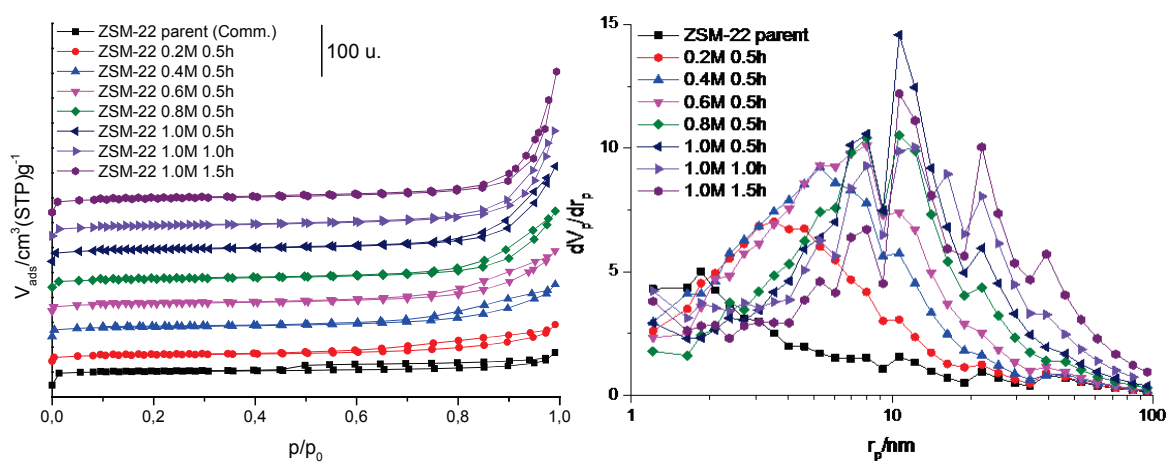

Figure 58: Isotherms from $\mathrm{N}_{2}$ physisorption of ZSM-22 left panel, corresponding mesopore distribution in the right panel.

The present work is in line with Verboekend et al.[60], who presented the importance of crystal morphology and aluminum distribution for mesopore introduction by desilication in ZSM-22. Also here the thin dimension of the rod/needle was described to be an obstacle. Hence both investigations concluded that morphology is important for the desilication process leading to mesopores (for ZSM-22), e.g. rods were more suitable than needles.

\section{Materials with strained structural units in combination with low acid site density are severely damaged (ITQ-13 D4R)}

The influence of strained structural units is interesting to evaluate with respect to the structural stability in alkaline media. In this respect the strained D4R in ITQ-13 could give an indication of its importance to desilication $(0.2 \mathrm{M}-1.0 \mathrm{M}$ for $0.5-1.5 \mathrm{~h}$ in water baths at $70^{\circ} \mathrm{C}$ ). The ITQ-13 sample treated (plate-like morphology) also had a high ( $\left.\mathrm{Si}+\mathrm{Ge}\right) / \mathrm{Al}$ ratio $>100$. The results clearly showed that the high $(\mathrm{Si}+\mathrm{Ge}) / \mathrm{Al}$ ratio combined with strained structural units lead to uncontrolled dissolution and rapid loss of material. Even after $0.2 \mathrm{M} \mathrm{NaOH}$ treatment for $0.5 \mathrm{~h} 27 \%$ of the material (not corrected for handling) was lost. Solutions with $0.4 \mathrm{M}, 0.6 \mathrm{M}$ and $0.8 \mathrm{M} \mathrm{NaOH}$ gave $45 \%, 68 \%$ and $81 \%$ material loss respectively which illustrates the rapid loss under treatment. The isotherms had no hysteresis and the BJH plots showed no sign of mesopores. The clear material destruction after $\mathrm{NaOH}$ attack is shown in Figure 59. 


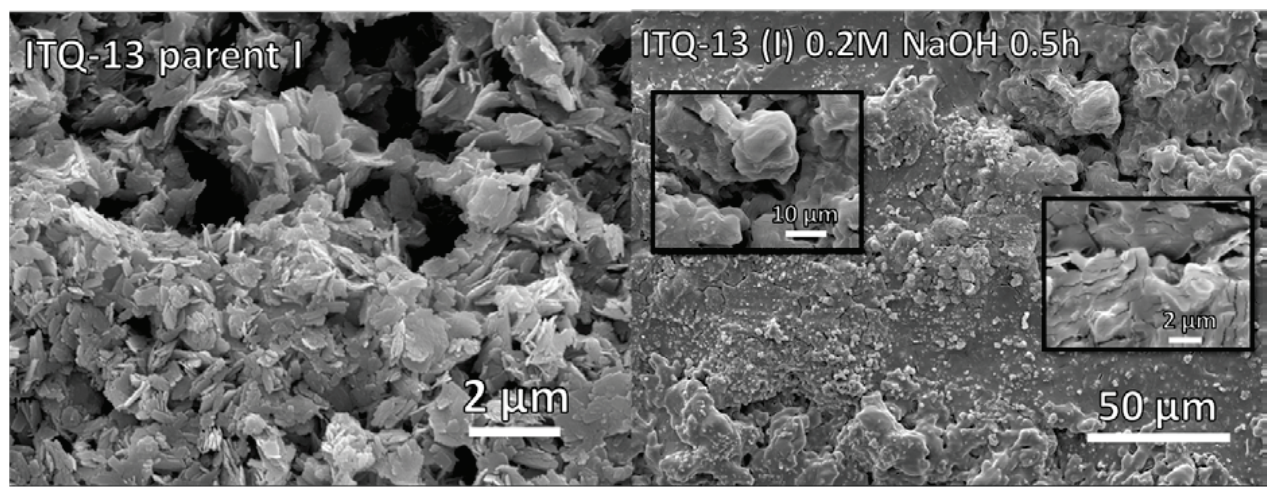

Figure 59: Parent and alkaline treated ITQ-13. Low concentrations of $\mathrm{NaOH}$ caused severe damage on the crystals.

Materials with high acid site density may easily dissolve if it contains a high density of defects (EU-1 and ZSM-57)

An EU-1 sample with $\mathrm{Si} / \mathrm{Al}$ ratio 16 and small crystals mainly of elliptical shape with rough defect rich surfaces (see Figure 60) were treated in alkaline solutions.

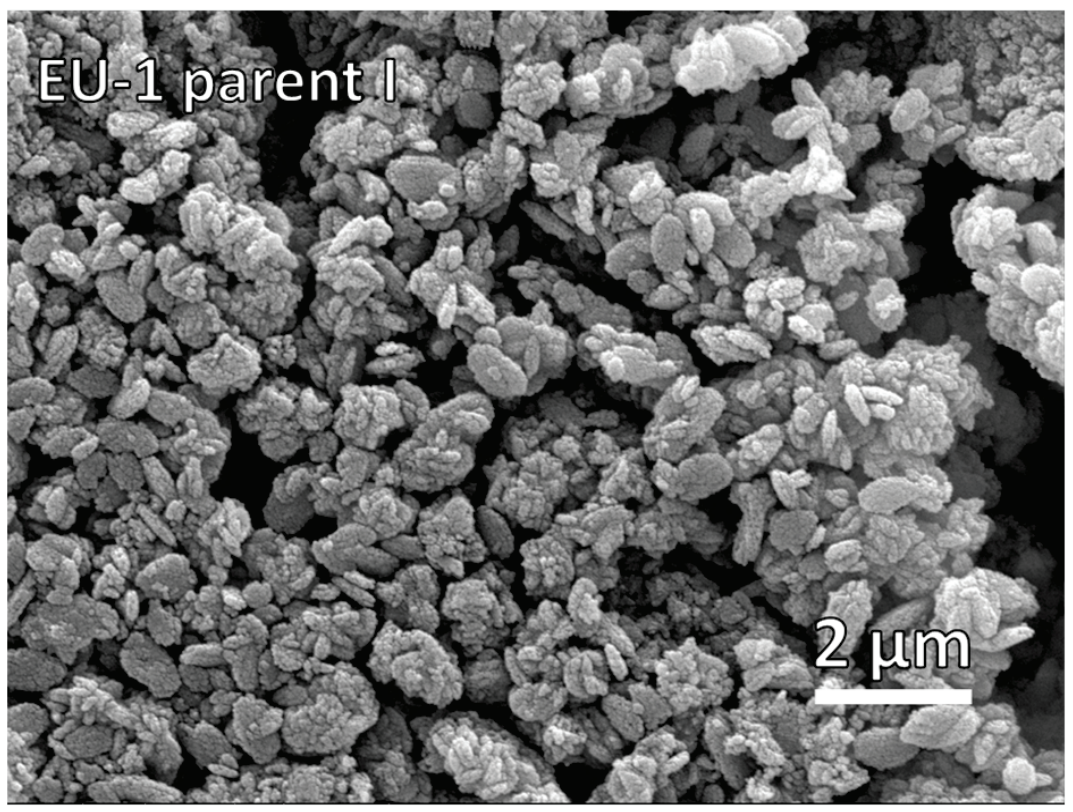

Figure 60: SEM micrographs of parent EU-1. Larger crystals tend to be agglomerates of smaller nanosized crystals. 
All crystals tended to be agglomerates of nanosized crystals. Even mild alkaline treatment ( 0.2 or $0.4 \mathrm{M} \mathrm{NaOH}$ for $0.5 \mathrm{~h}$ ) led to dramatic loss of surface area and close to eliminated micropore volume $\left(0.13-0.02 \mathrm{~cm}^{3} / \mathrm{g}\right)$. At the same time the mesopore volume was reduced or kept at initial levels. The large external surface area of the small nanosized particles result in a high number of points on the crystal in which the base can attack and extract $\mathrm{Si}$. Clearly even $\mathrm{Si} / \mathrm{Al}$ ratios as low as 16 can not hinder rapid dissolution of this particular material. Also in the case of ZSM-57 which also had particles which tended to be agglomerates of nanosized crystals the surface was rough. SEM micrographs showed that the crystals were clearly affected by the treatment. With respect to the presented conclusion it would be of interest to perform framework stability calculations, especially of EU-1, to investigate if the rapid dissolution can be ascribed to lower framework stability rather than surface structures.

\section{Materials with Si/Al ratios out of the optimal range observed for ZSM-5 may result in mesopores (TNU-9 and IM-5)}

For TNU-9 $(\mathrm{Si} / \mathrm{Al}=15)$ nitrogen physisorption data showed a steadily increase in the external surface area (from $24 \mathrm{~m}^{2} / \mathrm{g}$ to $139 \mathrm{~m}^{2} / \mathrm{g}$ ) and mesopore volume (from $0.14 \mathrm{~cm}^{3} / \mathrm{g}$ to $0.47 \mathrm{~cm}^{3} / \mathrm{g}$ ) with increasing $\mathrm{NaOH}$ concentrations, as shown in Table 27. The BET surface areas showed a slight drop initially after treatment.

Table 27: Results from nitrogen sorption measurements of parent, alkaline and combined alkaline surfactant treatment of TNU-9.

\begin{tabular}{|c|c|c|c|c|c|c|c|}
\hline \multicolumn{8}{|c|}{ TNU-9 } \\
\hline Sample & Time (h) & $\mathrm{S}_{\mathrm{BET}}\left(\mathrm{m}^{2} / \mathrm{g}\right)$ & $\mathrm{S}_{\mathrm{ext}}\left(\mathrm{m}^{2} / \mathrm{g}\right)^{\mathrm{a}}$ & $\begin{array}{c}\mathrm{S}_{\text {Micro }} \\
\left(\mathrm{m}^{2} / \mathrm{g}\right)^{\mathrm{b}}\end{array}$ & $\mathrm{V}_{\text {Total }}\left(\mathrm{cm}^{3} / \mathrm{g}\right)^{\mathrm{c}}$ & $\mathrm{V}_{\text {Micro }}\left(\mathrm{cm}^{3} / \mathrm{g}\right)^{\mathrm{a}}$ & $V_{\text {Meso }}\left(\mathrm{cm}^{2} / \mathrm{g}\right)^{d}$ \\
\hline Parent II & - & 481 & 24 & 457 & 0.32 & 0.18 & 0.14 \\
\hline Parent III & - & 457 & 28 & 429 & 0.32 & 0.18 & 0.15 \\
\hline (II) $0.8 \mathrm{M}$ & 0.5 & 443 & 72 & 371 & 0.45 & 0.15 & 0.30 \\
\hline (II) $1.0 \mathrm{M}$ & 0.5 & 476 & 95 & 381 & 0.50 & 0.16 & 0.34 \\
\hline (II) $1.0 \mathrm{M}$ & 1.0 & 475 & 127 & 348 & 0.57 & 0.14 & 0.43 \\
\hline (II) $1.0 \mathrm{M}$ & 1.5 & 465 & 139 & 326 & 0.61 & 0.14 & 0.47 \\
\hline $\begin{array}{l}\text { (III) } 0.25 \mathrm{M} \\
\mathrm{NaOH} 0.05 \mathrm{M} \\
\text { CTAB }\end{array}$ & 24 & 510 & 112 & 398 & 0.53 & 0.18 & 0.35 \\
\hline
\end{tabular}


Despite the low $\mathrm{Si} / \mathrm{Al}$ ratio, and small particles with smooth surfaces, TNU-9 was possible to desilicate with BJH-plots indicative of steadily larger pores developing from the micropore to the mesopore range as shown in Figure 61.

The results from sorption measurements of parent and treated IM-5 samples are shown in Figure 61. Samples treated with $0.8 \mathrm{M} \mathrm{NaOH}$ in water baths at $70^{\circ} \mathrm{C}$ for $0.5 \mathrm{~h}$ showed similar BET surface area (425 and $424 \mathrm{~m}^{2} / \mathrm{g}$ respectively) and micropore volume of 0.15 and $0.14 \mathrm{~cm}^{3} / \mathrm{g}$. The external surface area increased from $62 \mathrm{~m}^{2} / \mathrm{g}$ to $82 \mathrm{~m}^{2} / \mathrm{g}$ while the mesopore volume dropped from $0.40 \mathrm{~cm}^{3} / \mathrm{g}$ to $0.39 \mathrm{~cm}^{3} / \mathrm{g}$. For the harsher treatments of $1.0 \mathrm{M}$ for $0.5 \mathrm{~h}$ and $1.5 \mathrm{~h}$ increased the BET surface was $448 \mathrm{~m}^{2} / \mathrm{g}$ and 402 $\mathrm{m}^{2} / \mathrm{g}$ respectively. Also for these samples the micropore volume decreased from the parent material. However the mesopore volume increased from $0.40 \mathrm{~cm}^{3} / \mathrm{g}$ to $0.46 \mathrm{~cm}^{3} / \mathrm{g}$ (1.0M NaOH $0.5 \mathrm{~h})$ and $0.41 \mathrm{~cm}^{3} / \mathrm{g}(1.0 \mathrm{M} \mathrm{NaOH} 1.5 \mathrm{~h})$.

Table 28: Results from nitrogen sorption measurements of parent, alkaline and combined alkaline surfactant treatment of TNU-9.

\begin{tabular}{|c|c|c|c|c|c|c|c|}
\hline \multicolumn{8}{|c|}{ IM-5 } \\
\hline Sample & Time (h) & $\mathrm{S}_{\mathrm{BET}}\left(\mathrm{m}^{2} / \mathrm{g}\right)$ & $\mathrm{S}_{\mathrm{ext}}\left(\mathrm{m}^{2} / \mathrm{g}\right)^{\mathrm{a}}$ & $\mathrm{S}_{\text {Micro }}\left(\mathrm{m}^{2} / \mathrm{g}\right)^{\mathrm{b}}$ & $\mathrm{V}_{\text {Total }}\left(\mathrm{cm}^{3} / \mathrm{g}\right)^{\mathrm{e}}$ & $\begin{array}{l}V_{\text {Micro }} \\
\left(\mathrm{cm}^{3} / \mathrm{g}\right)\end{array}$ & $\begin{array}{l}V_{\text {Meso }} \\
\left(\mathrm{cm}^{2} / \mathrm{g}\right)\end{array}$ \\
\hline Parent II & - & 425 & 62 & 363 & 0.55 & 0.15 & 0.40 \\
\hline Parent III & - & 411 & 57 & 354 & 0.53 & 0.14 & 0.39 \\
\hline (II) $0.8 \mathrm{M}$ & 0.5 & 424 & 82 & 342 & 0.49 & 0.14 & 0.35 \\
\hline (II) $1.0 \mathrm{M}$ & 0.5 & 448 & 118 & 330 & 0.60 & 0.14 & 0.46 \\
\hline (II) $1.0 \mathrm{M}$ & 1.5 & 402 & 101 & 301 & 0.53 & 0.12 & 0.41 \\
\hline $\begin{array}{c}\text { (III) } 0.25 \mathrm{M} \\
\mathrm{NaOH} 0.05 \mathrm{M} \\
\text { CTAB }\end{array}$ & 24 & 438 & 134 & 304 & 0.68 & 0.14 & 0.54 \\
\hline
\end{tabular}

The even smaller particles of IM-5 relative to TNU-9 with similar low Si/Al ratio (14) was no hindrance for a steady increase in the pore size from micropore into the mesopore range. Actually both TNU-9 and IM-5 behave in much similar ways both with respect of shape of the isotherms and the BJH-plot as pores were developed from the border between the micropore and mesopore range. Similarities in crystal size, $\mathrm{Si} / \mathrm{Al}$ ratio, 
framework density and the fact that both structures contain mostly five-rings, but also four rings may be the cause of the similarities of these materials. The synthesis procedures and SDA is also almost identical which may result in many similar material properties. These results show that mesopores can be introduced even in zeolites with rather high acid site densities.
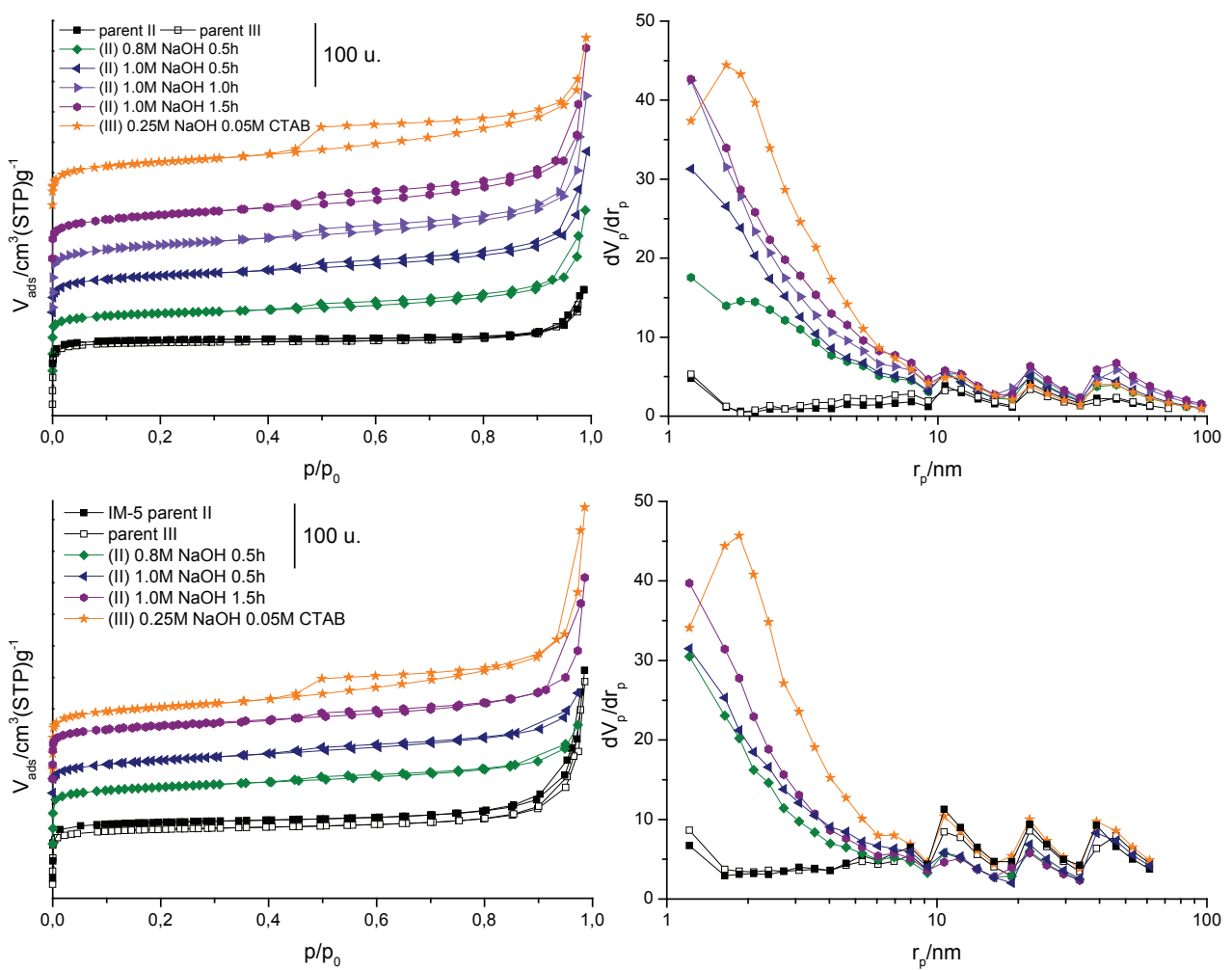

Figure 61: The $\mathrm{N}_{2}$-isotherms and mesopore distribution plot of the parent, alkaline treated TNU-9 (upper panel) and IM-5 (lower panel) samples are shown in the left and right panel respectively. The isotherms are vertically translated for clarity. The sample exposed to both $\mathrm{NaOH}(0.25 \mathrm{M})$ and surfactant $(0.05 \mathrm{M})$ showed the most pronounced hysteresis loop (left panel). BJH-plots are indicative of steadily increase in pore size.

\section{Surfactants combined with $\mathrm{NaOH}$ give superior protecting effect (hinders dissolution) and result in a narrower pore size distribution}

CTAB associated with $\mathrm{NaOH}$ has shown visible protecting effect on all zeolite samples treated in the present work. This could be a result of induced re-crystallization due to 
hydrophobicity. Recalling ITQ-13 which was severely damaged (27\% weight loss) after even mild treatment with $\mathrm{NaOH}\left(0.2 \mathrm{M} \mathrm{NaOH}, 0.5 \mathrm{~h} 70^{\circ} \mathrm{C}\right)$ only $35 \%$ was dissolved after $24 \mathrm{~h}$ tumbling in $0.25 \mathrm{M} \mathrm{NaOH}$ in the presence of $\mathrm{CTAB}$ at $80^{\circ} \mathrm{C}$. To mention one other example ZSM-57 showed approximately $60 \%$ weight loss when exposed to $1.0 \mathrm{M} \mathrm{NaOH}$ for $0.5 \mathrm{~h}$ at $70^{\circ} \mathrm{C}$ while $33 \%$ was lost after $24 \mathrm{~h}$ tumbling in $0.25 \mathrm{M} \mathrm{NaOH}$ in the presence of $0.05 \mathrm{M} \mathrm{CTAB}$. Even though the $\mathrm{NaOH}$ concentration of the static process is higher compared to the tumbling conditions, a higher weight loss would clearly be expected in the absence of surfactant. Figure 62 and Figure 63 show a high protecting effect from the surfactants as it partly dissolves the crystal, but reassemble the material around the micelles. Thus the crystals have a higher quality after surfactant treatment.

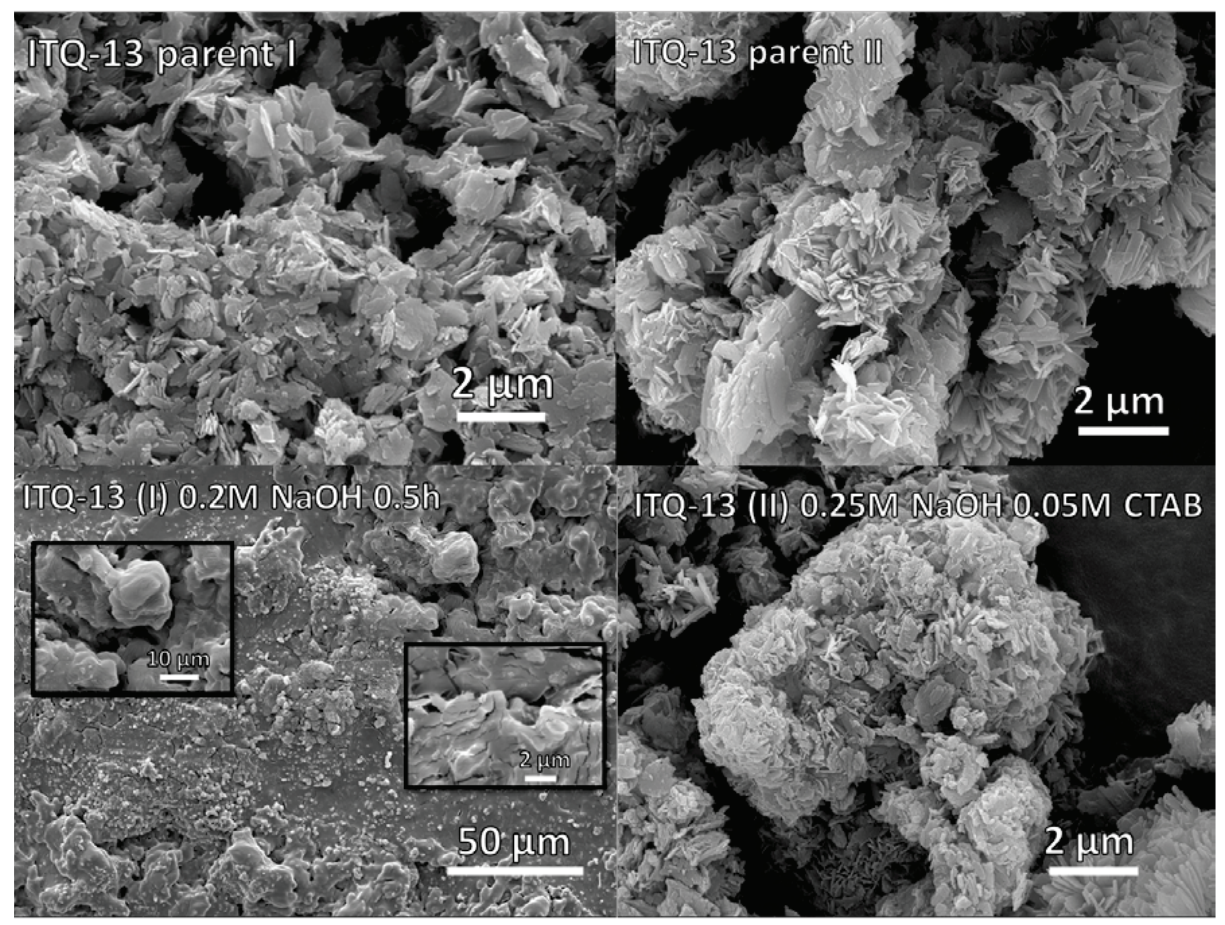

Figure 62: ITQ-13 treated with $0.2 \mathrm{M} \mathrm{NaOH}$ at $70^{\circ} \mathrm{C}$ for $0.5 \mathrm{~h}$ (down left panel) and with $0.25 \mathrm{M} \mathrm{NaOH}+0.05 \mathrm{M} \mathrm{CTAB}$ at tumbling conditions for $24 \mathrm{~h}$ at $80^{\circ} \mathrm{C}$. The surfactants protect the crystal from uncontrolled dissolution. 


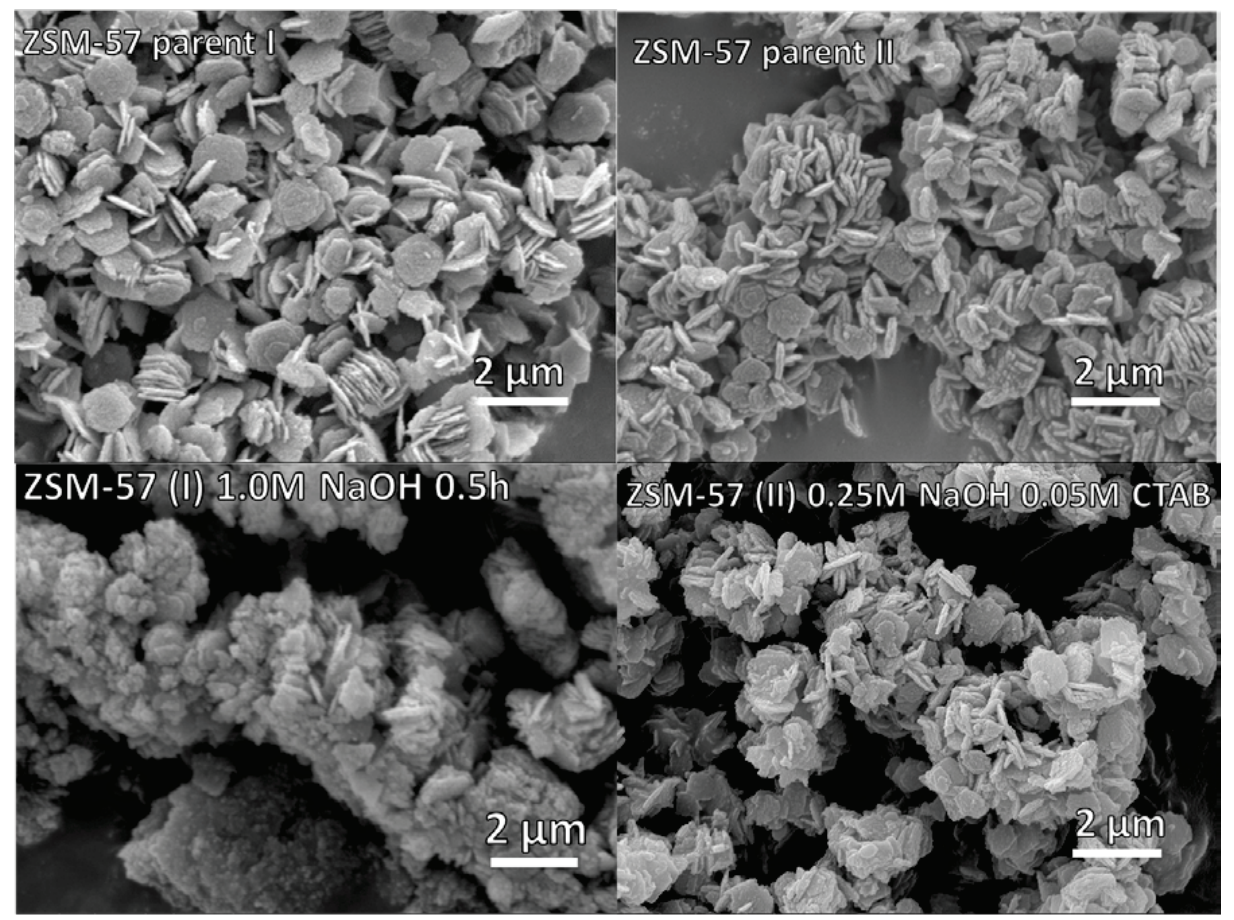

Figure 63: ZSM-57 treated with $0.2 \mathrm{M} \mathrm{NaOH}$ at $70^{\circ} \mathrm{C}$ for $0.5 \mathrm{~h}$ (down left panel) and with $0.25 \mathrm{M} \mathrm{NaOH}+0.05 \mathrm{M} \mathrm{CTAB}$ at tumbling conditions for $24 \mathrm{~h}$ at $80^{\circ} \mathrm{C}$.

The obtained results tend to show that CTAB results in a narrower pore size distribution than pure desilication likely caused by the micelle organization and reassembly of dissolved zeolite fragments in addition to the protective effect already mentioned. The indications in the BJH-plots are supported by TEM micrographs e.g. shown in Figure 64 (TNU-9 and IM-5). Both micrographs show presence of mesopores, typically around 10 $\mathrm{nm}$ in diameter. This is slightly larger than indicated from BJH-plots (see Figure 61). Further support can be found among the micrographs and description presented in paper V. 

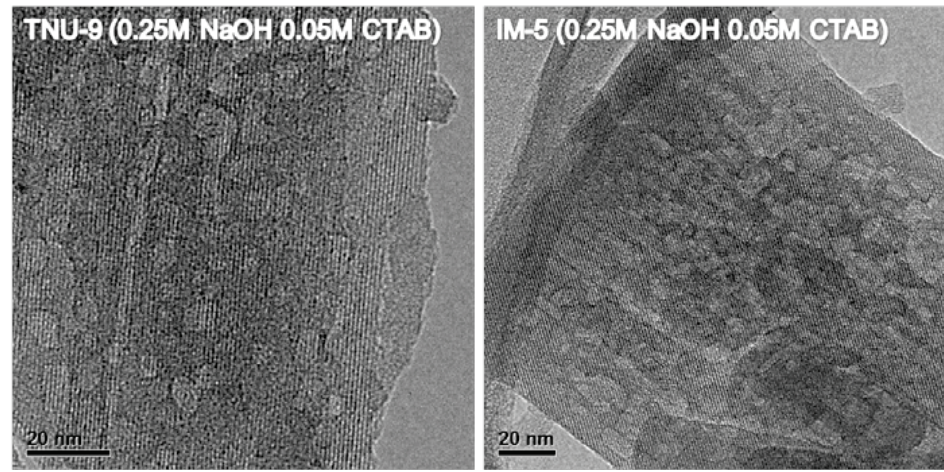

Figure 64: TEM micrographs of TNU-9 and IM-5 after surfactant treatment (24 h tumbling at $\left.80^{\circ} \mathrm{C}\right)$.

\section{Mesopore introduction does not necessary lead to improved lifetime in MTH}

Improved lifetimes were found for many of the herein tested samples after treatment. Slight improvement after desilication was observed both for TNU-9 and IM-5 as shown in Figure 65. It is very interesting to note that the surfactant treated samples gave much longer lifetime compared to the pure alkaline treatment. The lifetime increased with 125 $\%$ and $30 \%$ for TNU-9 and IM-5 respectively (extrapolation) after surfactant treatment relative to the parent materials.
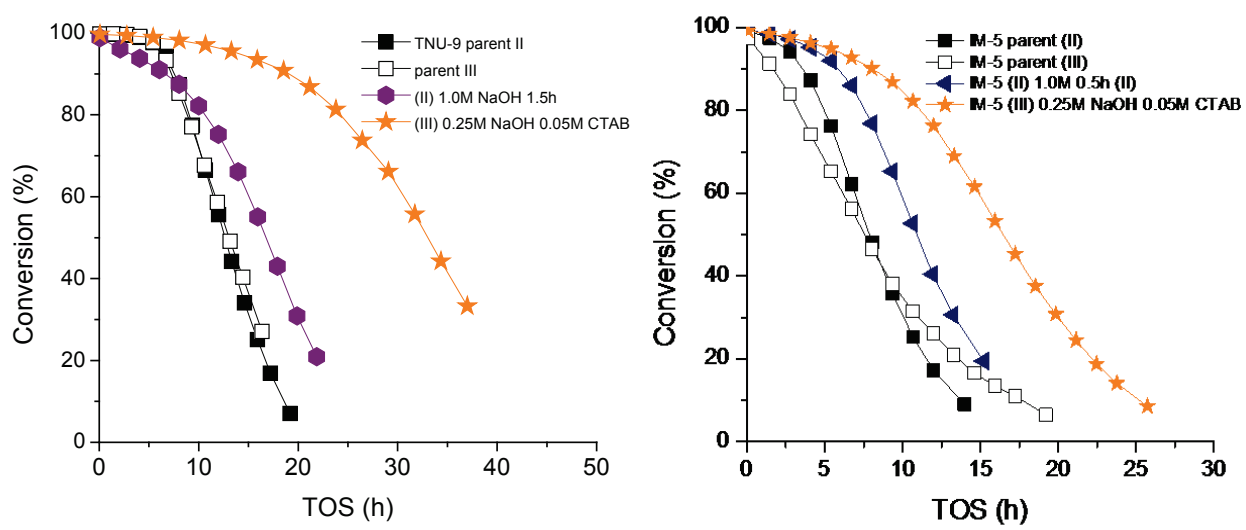

Figure 65: A comparison of the lifetimes of parent, alkaline treated and combined alkaline surfactant treated samples of TNU-9 and IM-5. TNU9: squares: parent, hexagons: zeolite treated in $1.0 \mathrm{M} \mathrm{NaOH}$ for $1.5 \mathrm{~h}$ at $70^{\circ} \mathrm{C}$, stars: Sample exposed to combined $\mathrm{NaOH}(0.25 \mathrm{M})$ and surfactant (0.05M CTAB) treatment for $24 \mathrm{~h}$ under tumbling conditions at $80^{\circ} \mathrm{C}(24$ 
h). IM-5: Closed and open squares: parent 1 and 2 respectively,

triangles: zeolite treated in $1.0 \mathrm{M} \mathrm{NaOH}$ for $0.5 \mathrm{~h}$ at $70^{\circ} \mathrm{C}$, stars $0.25 \mathrm{M}$

$\mathrm{NaOH}$ and 0.05M CTAB under tumbling conditions at $80^{\circ} \mathrm{C}(24 \mathrm{~h})$. All

reactions were carried out at $400^{\circ} \mathrm{C}$ and $W H S V=2 h^{-1}$.

Apparently for ZSM-57 pure alkaline treatment even lead to reduced lifetime over ZSM57 in MTH. By contrast the surfactant treated samples showed extraordinary improvement with more than $300 \%$ lifetime increase relative to the parent material as shown in Figure 66.
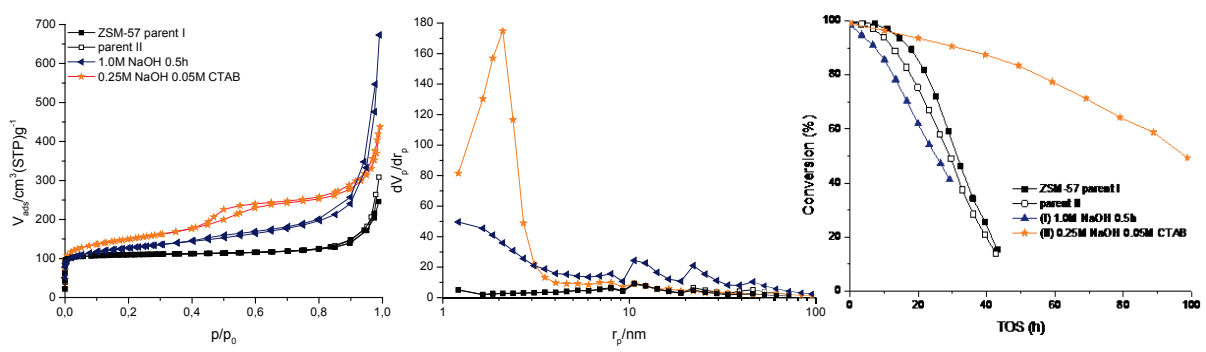

Figure 66: ZSM-57: isotherms, BJH-plot and conversion vs. time on stream.

Several materials also showed negative results after treatment. Both ZSM-22, ZSM-23, EU-1 gave overall reduced lifetime in MTH, even after surfactant treatment (except ZSM22 with similar conversion capacitates as the parent material). Common to these materials were a loss in micropore volume. In the case of EU the crystals were clearly damaged, but even in cases with less damaged crystals loss in micropore volume was observed. Verboekend showed a similar tendency after alkaline treatment of ZSM-22, but after performing acid wash $(\mathrm{HCl})$ the micropore was again restored. [60] Hence the reduced lifetime is likely a result of extra framework Al blocking the micropores essential for the activity of these materials. This implies that if acid wash was performed of the herein treated samples a higher methanol conversion capacity is expected (continuing work). Why TNU-9 and IM-5 showed improved lifetime in MTH after pure alkaline treatment when apparently the micropore volume was reduced is not obvious, but a 3D material with initial higher micropore volume would be expected to be less vulnerable to pore blocking as there are several entries/exits to the crystal than compared to 1D channel systems. 


\subsection{Main conclusions}

- The comparison of ZSM-22, ZSM-23, ZSM-48 and EU-1 showed that small differences in the channel sizes can to a large extent change the product distribution.

- ZSM-22 and ZSM-23 gave extraordinary selectivity towards non aromatic $\mathrm{C}_{5+}$ hydrocarbons (gasoline range). The larger channel of ZSM-48 gave higher aromatic content in the gas effluent. Despite narrower channels in EU-1 compared to the other structures the catalyst yielded aromatics. The unexpected behaviour indicated that the side pockets are involved in the MTH reaction over EU-1.

- ZSM-22, ZSM-23 and EU-1 showed selectivities independent of coke.

- ITQ-13 was, to the best of our knowledge, for the first time tested in MTH and found to be an active catalyst for the process.

- The catalyst $((\mathrm{Si}+\mathrm{Ge}) / \mathrm{Al}=42$, needle shaped crystals $)$ yielded a gas product spectrum intermediate of ZSM-22 (non aromatic) and the archetype ZSM-5. ITQ-13 had similar product cut-off as ZSM-5, with tetraMB as the heaviest significant product, but the amount of aromatics was clearly lower.

- Samples with a lower acid site density $((\mathrm{Si}+\mathrm{Ge}) / \mathrm{Al}>100$, plate-like morphology) gave a product spectrum very similar to ZSM-22. Thus, the amount of aromatics was low $(\sim 2 \%)$. The low acid site density lead to initial conversion of $70 \%$, but with a cumulative capacity well exceeding all results obtained over ZSM-22.

- Increased pressure (ITQ-13 (N) at $350^{\circ} \mathrm{C}$, WHSV $=2 \mathrm{~h}^{-1}, 30$ bars) gave a clear increase in the $\mathrm{C}_{5+}$ hydrocarbons (gasoline range), but the aromatic fraction in the gasoline range significantly increased while there was a tenfold decrease in methanol conversion capacity.

- ITQ-13 showed selectivity independent of coke.

- TNU-9 and IM-5 was tested for the first time as catalyst in MTH, both was found to be active. 
- The two structures could be synthesized in a narrow window of gel compositions. Turns easily into competing phases by slight differences in composition (e.g. $\mathrm{NaOH}$ and $\mathrm{Al}$ amount) when using 1,4-MPB and $\mathrm{HMBr}_{2}$ (TNU-9) and 1,5-MPP (IM-5). (Attempt using the fluoride route with $\mathrm{OH}^{-}$ ion exchanged SDA, Ge incorporation did not lead to the right phase for the compositions tested)

- Comparison of ZSM-11, ZSM-5, TNU-9 and IM-5 (zeolites with differing volumes at channel intersections) showed strikingly similar gas effluent selectivities, but TNU-9 and IM-5 had clearly lower catalytic stability compared with ZSM-5 and ZSM-11. The deactivation behaviour showed a clear correlation to the entrapped species in the respective pore systems. Hence we concluded that the larger aromatic species able to form in the larger cavities of TNU-9 and IM-5 was responsible for the rapid deactivation.

- In situ Raman studies of ZSM-5, TNU-9 and IM-5 showed more rapid coke development in TNU-9 and IM-5 compared with ZSM-5

- MS data indicated that initial pulses of methanol were consumed to build up hydrocarbon pool species. After prolonged pulse hydrocarbons in the product effluent decreased slower after cutting the methanol feed compared with the initial pulses of methanol (non quantitative preliminary results)

- It was difficult to assign the bands to specific coke precursors and cokelike species, but the results may indicate that non cyclic alkenes form first. Then they develop into larger alkenes that turn into aromatic/polyaromatic compounds.

- Lifetime improvement: Successful mesopore introduction by alkaline treatment $(\mathrm{NaOH})$ of zeolites is dependant of many parameters (topology and $\mathrm{T}$ atom stability, defects, acid site density/distribution, treatment conditions)

- It was not straightforward to range the most important to the least important parameters. However some combinations of parameters were not working, e.g. ITQ-13 with $\mathrm{Si} / \mathrm{Al}$ ratio $>100$ combined with strained D4R units lead to severe material loss. 
- A zeolite, EU-1 was easily dissolved despite a Si/Al ratio as low as 16 . We concluded that the defect rich surfaces (contained also 4-rings) led to uncontrolled dissolution. Hence a high density of Al is not necessary leading to inertness.

- ZSM-22 with needle shaped morphology did not lead to any mesopores by either of the following methods: $\mathrm{NaOH}$ treatment, Carbon Black or sucrose method.

- Mesopores were successfully introduced to ZSM-22 when the morphology changed from thin needles to rod-like crystals. Hence morphology is one important parameter obtaining mesopores.

- Introduction of mesopores is not necessary leading to improved catalytic performance in MTH. The catalytic stability clearly dropped after alkaline treatment of ZSM-22, EU-1, ZSM-57 and ZSM-23. Reduced lifetime was typically associated with a loss in micropore volume.

- Mesopores were successfully introduced in TNU-9, IM-5, ZSM-57 and ZSM-5. The lifetime improved for the two former structures (see details in paper $\mathrm{V}$ for the latter two).

- Lifetime improvement by combined alkaline and surfactant reassembly processes.

- More uniform mesopores were obtained with this method compared to pure alkaline treatment.

- Surfactants showed superior protecting effect - less destroyed crystals.

- Extraordinarily improved conversion capacity for ZSM-57 (lifetime increased $\sim 300 \%$ ), clear increase observed for TNU-9 and IM-5 (lifetime increased with $125 \%$ and $30 \%$ respectively).

- ZSM-57 (2D channel system consisting of 8-rings connected to 10-rings) was, to the best of our knowledge, tested as catalyst in MTH for the first time.

- The crystals were plate-like with 10-rings going in the short direction and 8-rings along the long direction. The gas effluent products were clearly dominated by the 10-ring. (this work is part of an extended study of "Morphology induced shape selectivity in zeolite catalysis" not yet published) 


\subsection{Suggestions for further work}

\section{Fundamental studies of the zeolite synthesis systems}

Despite many years of MTH research still there are questions needed to be answered. One specific goal is to gain further understanding on how zeolites deactivate. In this thesis it has been shown that even small differences in channel dimensions could lead to clearly different selectivities. Hence to establish mechanistic understanding of structureselectivity and structure-deactivation relations is important. An underlying challenge is here the complex synthesis system which often leads to many parameter studies. Hence fundamental studies of the synthesis systems which could lead to materials where only one parameter is varied at the time will also result in simplifying the discussion with respect to lifetime and selectivity in $\mathrm{MTH}$ as the influence of other parameters could be excluded. One suggestion for further work is to try to synthesise 10-ring zeolites with the fluoride route (and $\mathrm{OH}^{-}$ion-exchanged SDA as all of them may be synthesised by connecting trimethylamines with dibromo chains, SDA stable also as $\mathrm{OH}$-versions) witch may allow to synthesise the materials with a broader $\mathrm{Si} / \mathrm{Al}$ ratio range, hopefully without changing other parameters (example on successful expansion of $\mathrm{Si} / \mathrm{Al}$ ratio by the fluoride route keeping similar morphology: [43]).

\section{Further understanding of deactivation by developing new in situ techniques}

Overall trends in coke development in MTH could be followed with in situ Raman spectroscopy in MTH. Still the method could be developed further and be applied to materials which accommodate different hydrocarbon pool species (SAPO-34 (mainly aromatic hydrocarbon pool species), ZSM-22 (aromatic part of the proposed dual cycle expected to be turned off) and ZSM-5(both alkene and aromatic cycle believed to operate)). Maybe these differences (from isotopic labelling experiments) could be detectable with Raman spectroscopy and give insight in existing differences with respect to coke development.

\section{Lifetime}

Despite promising selectivities obtained over ZSM-22 and ZSM-23 in MTH the lifetime was very short. Herein we aimed at improving lifetime with different methods, especially 
with $\mathrm{NaOH}$ and combined $\mathrm{NaOH}$ and surfactant treatment, but found no improvement, rather the opposite. It is still a question why the lifetime is so short. In house synthesised ZSM-23 with in house synthesised SDA (recipe in section 4.1) showed clear improvement compared with the commercial ZSM-23 sample applied from Zeolyst (not shown). Hence the in house ZSM-23 sample also lived longer than the treated samples of the same structure. It would be of interest to look closer to why "ZSM-23" not always can be said to be "ZSM-23". Even the topology is similar other parameters may change as morphology and acid site location and density, and severely influence the catalytic stability. Pore mouth catalysis would be interesting to elaborate for these structures.

\section{Develop the work aiming at creating mesopores further towards one parameter study}

It was difficult to obtain one parameter study of the materials exposed to alkaline and alkaline and surfactants. Hence a deepening in the individual materials approaching one parameter study would be interesting. However it may require large effort to synthesise materials with only one parameter varying at the time as described above (and based on our experience with many trials to expand the $\mathrm{Si} / \mathrm{Al}$ ratios of herein presented structures). Also the treatment conditions should be extended and optimised for each material both with and without surfactants present in the $\mathrm{NaOH}$ solution.

\section{Acid wash of desilicated materials}

Acid wash of alkaline treated samples and samples treated with $\mathrm{NaOH}$ and surfactant should be exposed to acids to investigate the effect of eventual Al removal from the pore system. Work on 1D zeolites ZSM-22 and ZSM-23 is already started by another PhD. student in our group, and has led to positive results.

\section{Morphology induced shape selectivity in zeolite catalysis}

Preliminary results indicate that if the crystal size and shape is changed with respect to the channel system (e.g. in 2D zeolite pore systems with 8-ring in one direction and 10-ring in another the selectivity may be manipulated by growing the crystal along one channel restricting growth one the other dimension and vice versa). ZSM-57 among other materials is part of this project (manuscript in preparation). 


\section{References}

[1] G.A. Somorjai, J.Y. Park, Molecular Factors of Catalytic Selectivity, Angew Chem Int Edit, 47 (2008) 9212-9228.

[2] J.A. Rabo, M.W. Schoonover, Early discoveries in zeolite chemistry and catalysis at Union Carbide, and follow-up in industrial catalysis, Appl Catal a-Gen, 222 (2001) 261-275.

[3] D.S. Coombs, A. Alberti, T. Armbruster, G. Artioli, C. Colella, E. Galli, J.D. Grice, F. Liebau, J.A. Mandarino, H. Minato, E.H. Nickel, E. Passaglia, D.R. Peacor, S. Quartieri, R. Rinaldi, M. Ross, R.A. Sheppard, E. Tillmanns, G. Vezzalini, Recommended nomenclature for zeolite minerals: Report of the subcommittee on zeolites of the international mineralogical association, commission on new minerals and mineral names, Eur. J. Mineral. , 10 (1998) 1037-1081.

[4] D.J. Earl, M.W. Deem, Toward a database of hypothetical zeolite structures, Ind Eng Chem Res, 45 (2006) 5449-5454.

[5] D.J. Earl, M.W. Deem, Generation of a database of hypothetical zeolite structures, Abstr Pap Am Chem S, 237 (2009).

[6] J. Weitkamp, Zeolites and catalysis, Solid State lonics, 131 (2000) 175-188.

[7] A. Corma, J.X. Jiang, J.H. Yu, Extra-Large-Pore Zeolites: Bridging the Gap between Micro and Mesoporous Structures, Angew Chem Int Edit, 49 (2010) 3120-3145.

[8] B. Smit, T.L.M. Maesen, Molecular simulations of zeolites: Adsorption, diffusion, and shape selectivity, Chem Rev, 108 (2008) 4125-4184.

[9] J. Perez-Ramirez, J.C. Groen, A. Bruckner, M.S. Kumar, U. Bentrup, M.N. Debbagh, L.A. Villaescusa, Evolution of isomorphously substituted iron zeolites during activation: comparison of Fe-beta and Fe-ZSM-5, J Catal, 232 (2005) 318-334.

[10] H. Yahiro, M. Iwamoto, Copper ion-exchanged zeolite catalysts in deNO(x) reaction, Appl Catal a-Gen, 222 (2001) 163-181.

[11] S. Sato, H. Hirabayashi, H. Yahiro, N. Mizuno, M. Iwamoto, Iron Ion-Exchanged Zeolite - the Most Active Catalyst at 473-K for Selective Reduction of Nitrogen Monoxide by Ethene in Oxidizing Atmosphere, Catal. Lett., 12 (1992) 193-200.

[12] A. lijima, Geology of Natural Zeolites and Zeolitic Rocks, Pure and Applied Chemistry, 52 (1980) 2115-2130.

[13] M.E. Davis, R.F. Lobo, Zeolite and Molecular-Sieve Synthesis, Chem Mater, 4 (1992) 756-768. [14] C.S. Cundy, P.A. Cox, The hydrothermal synthesis of zeolites: Precursors, intermediates and reaction mechanism, Micropor. Mesopor. Mater., 82 (2005) 1-78.

[15] M.A. Camblor, L.A. Villaescusa, M.J. Díaz-Cabañas, Synthesis of all-silica and high-silica molecular sieves in fluoride media, Top. Catal., 9 (1999) 59-76.

[16] R.M. Barrer, Zeolites and their synthesis, Zeolites, 1 (1981) 130-140.

[17] R.M. Barrer, P.J. Denny, Hydrothermal Chemistry of Silicates .9. Nitrogenous Aluminosilicates, J Chem Soc, (1961) 971.

[18] Y. Kubota, M.M. Helmkamp, S.I. Zones, M.E. Davis, Properties of organic cations that lead to the structure-direction of high-silica molecular sieves, Micropor. Mesopor. Mater., 6 (1995) 213229.

[19] R. Castaneda, A. Corma, V. Fornes, J. Martinez-Triguero, S. Valencia, Direct synthesis of a $9 x$ 10 member ring zeolite (AI-ITQ-13): A highly shape-selective catalyst for catalytic cracking, J

Catal, 238 (2006) 79-87.

[20] S.H. Lee, C.H. Shin, D.K. Yang, S.D. Ahn, I.S. Nam, S.B. Hong, Reinvestigation into the synthesis of zeolites using diquaternary alkylammonium ions $(\mathrm{CH} 3)(3) \mathrm{N}+(\mathrm{CH} 2)(\mathrm{n}) \mathrm{N}+(\mathrm{CH} 3)(3)$ with $n=3-10$ as structure-directing agents, Micropor. Mesopor. Mater., 68 (2004) 97-104. 
[21] S.B. Hong, Use of flexible diquaternary structure-directing agents in zeolite synthesis: Discovery of zeolites TNU-9 and TNU-10 and their catalytic properties, Catal. Surv. Asia, 12 (2008) 131-144.

[22] S.H. Lee, D.K. Lee, C.H. Shin, Y.K. Park, P.A. Wright, W.M. Lee, S.B. Hong, Synthesis, characterization, and catalytic properties of zeolites IM-5 and NU-88, J. Catal., 215 (2003) 151170.

[23] A. Corma, F. Rey, J. Rius, M.J. Sabater, S. Valencia, Supramolecular self-assembled molecules as organic directing agent for synthesis of zeolites, Nature, 431 (2004) 287-290.

[24] C.T. Brigden, D. Thompsett, C.D. Williams, Preparation of isomorphously substituted scandium ZSM-5 using aqueous fluoride gels, Dalton T, (2004) 2829-2830.

[25] J.L. Guth, H. Kessler, J.M. Higel, J.M. Lamblin, J. Patarin, A. Seive, J.M. Chezeau, R. Wey, Zeolite Synthesis in the Presence of Fluoride Ions - a Comparison with Conventional Synthesis Methods, Acs Sym Ser, 398 (1989) 176-195.

[26] Y.J. Wu, J. Wang, P. Liu, W. Zhang, J. Gu, X.J. Wang, Framework-Substituted Lanthanide MCM-22 Zeolite: Synthesis and Characterization, J Am Chem Soc, 132 (2010) 17989-17991. [27] G.O. Brunner, W.M. Meier, Framework Density Distribution of Zeolite-Type Tetrahedral Nets, Nature, 337 (1989) 146-147.

[28] Hypothetical Zeolite Database, http://mezeopor.jlu.edu.cn/hypo/,

[29] T. Blasco, A. Corma, M.J. Diaz-Cabanas, F. Rey, J.A. Vidal-Moya, C.M. Zicovich-Wilson, Preferential location of $\mathrm{Ge}$ in the double four-membered ring units of ITQ-7 zeolite, J. Phys. Chem. B, 106 (2002) 2634-2642.

[30] G. Sastre, J.A. Vidal-Moya, T. Blasco, J. Rius, J.L. Jorda, M.T. Navarro, F. Rey, A. Corma, Preferential location of $\mathrm{Ge}$ atoms in polymorph $\mathrm{C}$ of beta zeolite (ITQ-17) and their structuredirecting effect: A computational, XRD, and NMR spectroscopic study, Angew Chem Int Edit, 41 (2002) 4722-4726.

[31] G. Sastre, A. Pulido, R. Castaneda, A. Corma, Effect of the germanium incorporation in the synthesis of EU-1, ITQ-13, ITQ-22, and ITQ-24 zeolites, J Phys Chem-Us, 108 (2004) 8830-8835. [32] J.X. Jiang, J.L. Jorda, M.J. Diaz-Cabanas, J.H. Yu, A. Corma, The Synthesis of an Extra-LargePore Zeolite with Double Three-Ring Building Units and a Low Framework Density, Angew Chem Int Edit, 49 (2010) 4986-4988.

[33] A. Corma, M.J. Diaz-Cabanas, J. Jiang, M. Afeworki, D.L. Dorset, S.L. Soled, K.G. Strohmaier, Extra-large pore zeolite (ITQ-40) with the lowest framework density containing double four- and double three-rings, P Natl Acad Sci USA, 107 (2010) 13997-14002.

[34] International Zeolite Association, 2012

[35] S. Teketel, W. Skistad, S. Benard, U. Olsbye, K.P. Lillerud, P. Beato, S. Svelle, Shape Selectivity in the Conversion of Methanol to Hydrocarbons: The Catalytic Performance of One-Dimensional 10-Ring Zeolites: ZSM-22, ZSM-23, ZSM-48, and EU-1, ACS Catal., 2 (2012) 26-37.

[36] J.L. Schlenker, J.B. Higgins, E.W. Valyocsik, The Framework Topology of ZSM-57 - a New Synthetic Zeolite, Zeolites, 10 (1990) 293-296.

[37] F. Gramm, C. Baerlocher, L.B. McCusker, S.J. Warrender, P.A. Wright, B. Han, S.B. Hong, Z. Liu, T. Ohsuna, O. Terasaki, Complex zeolite structure solved by combining powder diffraction and electron microscopy, Nature, 444 (2006) 79-81.

[38] C. Baerlocher, F. Gramm, L. Massuger, L.B. McCusker, Z.B. He, S. Hovmoller, X.D. Zou, Structure of the polycrystalline zeolite catalyst IM-5 solved by enhanced charge flipping, Science, 315 (2007) 1113-1116.

[39] A. Corma, M. Puche, F. Rey, G. Sankar, S.J. Teat, A zeolite structure (ITQ-13) with three sets of medium-pore crossing channels formed by 9- and 10-rings, Angew. Chem. Int. Ed. , 42 (2003) 1156-1159. 
[40] T. Boix, M. Puche, M.A. Camblor, A. Corma, Synthetic Porous Crystalline Material ITQ-13, its Synthesis and Use, 2002.

[41] U. Olsbye, S. Svelle, M. Bjorgen, P. Beato, T.V.W. Janssens, F. Joensen, S. Bordiga, K.P. Lillerud, Conversion of Methanol to Hydrocarbons: How Zeolite Cavity and Pore Size Controls Product Selectivity, Angew Chem Int Edit, 51 (2012) 5810-5831.

[42] B.B. Schaack, W. Schrader, F. Schuth, Structural Insight into Germanium-Containing Silicate Species by Electrospray lonization Mass Spectrometry (ESI-MS) and ESI-MS/MS, J Phys Chem B, 113 (2009) 11240-11246.

[43] E.A. Eilertsen, M.H. Nilsen, R. Wendelbo, U. Olsbye, K.P. Lillerud, Synthesis of high silica CHA zeolites with controlled Si/Al ratio, in: A. Gédéon, F. Babonneau, F. Massiani (Eds.) Zeolites and related materials: Trends, targets and challenges, Elsevier, Amsterdam, 2008, pp. 265-268.

[44] A.B. Pinar, C. Marquez-Alvarez, M. Grande-Casas, J. Perez-Pariente, Template-controlled acidity and catalytic activity of ferrierite crystals, J Catal, 263 (2009) 258-265.

[45] S. Teketel, L.L. F., W. Skistad, U. Olsbye, K.P. Lillerud, P. Beato, S. Svelle, Morphology Induced Shape Selectivity in Zeolite Catalysis, in prep.

[46] M. Choi, K. Na, J. Kim, Y. Sakamoto, O. Terasaki, R. Ryoo, Stable single-unit-cell nanosheets of zeolite MFI as active and long-lived catalysts (vol 461, pg 246, 2009), Nature, 461 (2009). [47] M. Hartmann, Hierarchical zeolites: A proven strategy to combine shape selectivity with efficient mass transport, Angew Chem Int Edit, 43 (2004) 5880-5882.

[48] M. Ogura, S.Y. Shinomiya, J. Tateno, Y. Nara, E. Kikuchi, H. Matsukata, Formation of uniform mesopores in ZSM-5 zeolite through treatment in alkaline solution, Chem Lett, (2000) 882-883. [49] M. Ogura, S.Y. Shinomiya, J. Tateno, Y. Nara, M. Nomura, E. Kikuchi, M. Matsukata, Alkalitreatment technique - New method for modification of structural and acid-catalytic properties of ZSM-5 zeolites, Appl Catal a-Gen, 219 (2001) 33-43.

[50] W.C. Yoo, X.Y. Zhang, M. Tsapatsis, A. Stein, Synthesis of mesoporous ZSM-5 zeolites through desilication and re-assembly processes, Micropor. Mesopor Mater., 149 (2012) 147-157. [51] F. Schmidt, M.R. Lohe, B. Büchner, F. Giordanino, F. Bonino, S. Kaskel, Improved catalytic performance of hierarchical ZSM-5 synthesized by desilication with surfactants. , Micropor. Mesopor. Mater., 165 (2013) 148-157.

[52] R. Le Van Mao, S.Y. Xiao, A. Ramsaran, J.H. Yao, Selective Removal of Silicon from Zeolite Frameworks Using Sodium-Carbonate, J Mater Chem, 4 (1994) 605-610.

[53] R. Le Van Mao, A. Ramsaran, S.Y. Xiao, J.H. Yao, V. Semmer, Ph of the Sodium-Carbonate Solution Used for the Desilication of Zeolite Materials, J Mater Chem, 5 (1995) 533-535.

[54] J.C. Groen, J.A. Moulijn, J. Perez-Ramirez, Alkaline posttreatment of MFI zeolites. From accelerated screening to scale-up, Ind Eng Chem Res, 46 (2007) 4193-4201.

[55] J.C. Groen, L.A.A. Peffer, J.A. Moulijn, J. Perez-Ramirez, Mechanism of hierarchical porosity development in MFI zeolites by desilication: The role of aluminium as a pore-directing agent, Chem-Eur J, 11 (2005) 4983-4994.

[56] J.C. Groen, J.C. Jansen, J.A. Moulijn, J. Perez-Ramirez, Optimal aluminum-assisted mesoporosity development in MFI zeolites by desilication, J Phys Chem B, 108 (2004) 1306213065.

[57] J. Perez-Ramirez, C.H. Christensen, K. Egeblad, C.H. Christensen, J.C. Groen, Hierarchical zeolites: enhanced utilisation of microporous crystals in catalysis by advances in materials design, Chem Soc Rev, 37 (2008) 2530-2542.

[58] J.C. Groen, S. Abello, L.A. Villaescusa, J. Perez-Ramirez, Mesoporous beta zeolite obtained by desilication, Micropor. Mesopor. Mater., 114 (2008) 93-102.

[59] S. Svelle, L. Sommer, K. Barbera, P.N.R. Vennestrom, U. Olsbye, K.P. Lillerud, S. Bordiga, Y.H. Pan, P. Beato, How defects and crystal morphology control the effects of desilication, Catal Today, 168 (2011) 38-47. 
[60] D. Verboekend, A.M. Chabaneix, K. Thomas, J.-P. Gilson, J. Pérez-Ramírez, Mesoporous ZSM22 zeolite obtained by desilication: peculiarities associated with crystal morphology and aluminium distribution, Cryst. Eng. Comm., 13 (2011) 3408-3416.

[61] D. Verboekend, J. Perez-Ramirez, Design of hierarchical zeolite catalysts by desilication, Catal. Sci. Technol., 1 (2011) 879-890.

[62] A.N.C. van Laak, R.W. Gosselink, S.L. Sagala, J.D. Meeldijk, P.E. de Jongh, K.P. de Jong, Alkaline treatment on commercially available aluminum rich mordenite, Appl Catal a-Gen, 382 (2010) 65-72.

[63] Y.P. Khitev, I.I. Ivanova, Y.G. Kolyagin, O.A. Ponomareva, Skeletal isomerization of 1-butene over micro/mesoporous materials based on FER zeolite, Appl Catal a-Gen, 441 (2012) 124-135.

[64] M. Kustova, K. Egeblad, K. Zhu, C.H. Christensen, Versatile route to zeolite single crystals with controlled mesoporosity: In situ sugar decomposition for templating of hierarchical zeolites, Chem. Mater., 19 (2007) 2915-2917.

[65] A.H. Janssen, I. Schmidt, C.J.H. Jacobsen, A.J. Koster, K.P. de Jong, Exploratory study of mesopore templating with carbon during zeolite synthesis, Micropor. Mesopor. Mater., 65 (2003) 59-75.

[66] A. Corma, Inorganic Solid Acids and Their Use in Acid-Catalyzed Hydrocarbon Reactions, Chem Rev, 95 (1995) 559-614.

[67] J. Kim, M. Choi, R. Ryoo, Effect of mesoporosity against the deactivation of MFI zeolite catalyst during the methanol-to-hydrocarbon conversion process, J Catal, 269 (2010) 219-228.

[68] R. Baur, R. Krishna, The effectiveness factor for zeolite catalysed reactions, Catal Today, 105 (2005) 173-179.

[69] S.M. Csicsery, Catalysis by Shape Selective Zeolites - Science and Technology, Pure and Applied Chemistry, 58 (1986) 841-856.

[70] Shell Gas-to-liquids (GTL), 2012

[71] C.D. Chang, A.J. Silvestri, The Conversion of Methanol and Other O-Compounds to Hydrocarbons over Zeolite Catalysts, J Catal, 47 (1977) 249-259.

[72] C.D. Chang, The New Zealand Gas-to-Gasoline plant: An engineering tour de force, Cat. Today 13 (1992) 103-111.

[73] F.J. Keil, Methanol-to-hydrocarbons: process technology, Micropor. Mesopor. Mater., 29 (1999) 49-66.

[74] J. Topp-Jørgensen, Tops $\varnothing$ Integrated Gasoline Synthesis - The Tigas Process, Stud. Surf. Sci. Cat., 36 (1988) 293-305.

[75] S. Kvisle, H.R. Nilsen, T. Fuglerud, A. Gronvold, P.R. Pujado, P.T. Barger, J.M. Andersen, Methanol to olefins (MTO). Abstr Pap Am Chem S, 223 (2002) U648-U648.

[76] S. Kvisle, T. Fuglerud, U. Olsbye, K.P. Lillerud, B.V. Vora, Methanol-to-Hydrocarbons, in: G. Ertl, H. Knozinger, J. Weitkamp (Eds.) Handbook of Heterogeneous Catalysis, Wiley-VCH Verlag GmbH, Weinheim, 2008, pp. 2950-2965.

[77] UOP Coal to plastics - 1st breakthrough, 2008.

[78] TIGAS - producing fuel from waste wood, 2012.

[79] ExxonMobil - Methanol to Gasoline (MTG) Technology, 2012.

[80] UOP Coal to plastics - 2nd breakthrough, 2012.

[81] UOP Coal to plastics - 3rd breakthrough 2012.

[82] UOP Coal to plastics - 4th breakthrough, 2013.

[83] J.L. White, Methanol-to-hydrocarbon chemistry: The carbon pool (r)evolution, Catal. Sci. Technol., 1 (2011) 1630-1635.

[84] W.G. Song, D.M. Marcus, H. Fu, J.O. Ehresmann, J.F. Haw, An Oft-studied reaction that may never have been: Direct catalytic conversion of methanol or dimethyl ether to hydrocarbons on the solid acids HZSM-5 or HSAPO-34, J Am Chem Soc, 124 (2002) 3844-3845. 
[85] M. Bjorgen, S. Svelle, F. Joensen, J. Nerlov, S. Kolboe, F. Bonino, L. Palumbo, S. Bordiga, U. Olsbye, Conversion of methanol to hydrocarbons over zeolite H-ZSM-5: On the origin of the olefinic species, J Catal, 249 (2007) 195-207.

[86] O. Mikkelsen, P.O. Ronning, S. Kolboe, Use of isotopic labeling for mechanistic studies of the methanol-to-hydrocarbons reaction. Methylation of toluene with methanol over H-ZSM-5, Hmordenite and H-beta, Micropor. Mesopor. Mater., 40 (2000) 95-113.

[87] B. Arstad, S. Kolboe, The reactivity of molecules trapped within the SAPO-34 cavities in the methanol-to-hydrocarbons reaction, J Am Chem Soc, 123 (2001) 8137-8138.

[88] B. Arstad, S. Kolboe, Methanol-to-hydrocarbons reaction over SAPO-34. Molecules confined in the catalyst cavities at short time on stream, Catal. Lett., 71 (2001) 209-212.

[89] S. Svelle, F. Joensen, J. Nerlov, U. Olsbye, K.P. Lillerud, S. Kolboe, M. Bjorgen, Conversion of methanol into hydrocarbons over zeolite H-ZSM-5: Ethene formation is mechanistically separated from the formation of higher alkenes, J Am Chem Soc, 128 (2006) 14770-14771. [90] S. Svelle, U. Olsbye, F. Joensen, M. Bjorgen, Conversion of methanol to alkenes over medium- and large-pore acidic zeolites: Steric manipulation of the reaction intermediates governs the ethene/propene product selectivity, J. Phys. Chem. C., 111 (2007) 17981-17984. [91] R.M. Dessau, On the H-Zsm-5 Catalyzed Formation of Ethylene from Methanol or Higher Olefins, J Catal, 99 (1986) 111-116.

[92] R.M. Dessau, R.B. Lapierre, On the Mechanism of Methanol Conversion to Hydrocarbons over Hzsm-5, J Catal, 78 (1982) 136-141.

[93] T. Mole, J.A. Whiteside, D. Seddon, Aromatic Co-Catalysis of Methanol Conversion over Zeolite Catalysts, J Catal, 82 (1983) 261-266.

[94] T. Mole, G. Bett, D. Seddon, Conversion of Methanol to Hydrocarbons over Zsm-5 Zeolite an Examination of the Role of Aromatic-Hydrocarbons Using Carbon-13-Labeled and DeuteriumLabeled Feeds, J Catal, 84 (1983) 435-445.

[95] B.E. Langner, Reactions of Methanol on Zeolites with Different Pore Structures, Appl Catal, 2 (1982) 289-302.

[96] I.M. Dahl, S. Kolboe, On the Reaction-Mechanism for Hydrocarbon Formation from Methanol over Sapo-34 .1. Isotopic Labeling Studies of the Co-Reaction of Ethene and Methanol, J Catal, 149 (1994) 458-464.

[97] I.M. Dahl, S. Kolboe, On the reaction mechanism for hydrocarbon formation from methanol over SAPO-34 .2. Isotopic labeling studies of the co-reaction of propene and methanol, J Catal, 161 (1996) 304-309.

[98] I.M. Dahl, S. Kolboe, On the Reaction-Mechanism for Propene Formation in the MTO Reaction over Sapo-34, Catal. Lett., 20 (1993) 329-336.

[99] M. Bjorgen, F. Joensen, K.P. Lillerud, U. Olsbye, S. Svelle, The mechanisms of ethene and propene formation from methanol over high silica H-ZSM-5 and H-beta, Catal Today, 142 (2009) 90-97.

[100] D. Lesthaeghe, A. Horre, M. Waroquier, G.B. Marin, V. Van Speybroeck, Theoretical Insights on Methylbenzene Side-Chain Growth in ZSM-5 Zeolites for Methanol-to-Olefin Conversion, Chem-Eur J, 15 (2009) 10803-10808.

[101] A. Sassi, M.A. Wildman, H.J. Ahn, P. Prasad, J.B. Nicholas, J.F. Haw, Methylbenzene chemistry on zeolite HBeta: Multiple insights into methanol-to-olefin catalysis, J Phys Chem B, 106 (2002) 2294-2303.

[102] A. Sassi, M.A. Wildman, J.F. Haw, Reactions of butylbenzene isomers on zeolite HBeta: Methanol-to-olefins hydrocarbon pool chemistry and secondary reactions of olefins, J Phys Chem B, 106 (2002) 8768-8773. 
[103] T. Xu, D.H. Barich, P.W. Goguen, W.G. Song, Z.K. Wang, J.B. Nicholas, J.F. Haw, Synthesis of a benzenium ion in a zeolite with use of a catalytic flow reactor, J Am Chem Soc, 120 (1998) 4025-4026.

[104] R.F. Sullivan, R.P. Sieg, G.E. Langlois, C.J. Egan, A New Reaction That Occurs in Hydrocracking of Certain Aromatic Hydrocarbons, J Am Chem Soc, 83 (1961) 1156-\&.

[105] M. Seiler, W. Wang, A. Buchholz, M. Hunger, Direct evidence for a catalytically active role of the hydrocarbon pool formed on zeolite H-ZSM-5 during the methanol-to-olefin conversion, Catal. Lett., 88 (2003) 187-191.

[106] M. Bjorgen, U. Olsbye, D. Petersen, S. Kolboe, The methanol-to-hydrocarbons reaction: insight into the reaction mechanism from [C-12] benzene and [C-13]methanol coreactions over zeolite H-beta, J Catal, 221 (2004) 1-10.

[107] M.W. Erichsen, S. Svelle, U. Olsbye, H-SAPO-5 as methanol-to-olefins (MTO) model catalyst: Towards elucidating the effects of acid strength, J Catal, 298 (2013) 94-101.

[108] S.B. Hong, H.K. Min, C.H. Shin, P.A. Cox, S.J. Warrender, P.A. Wright, Synthesis, crystal structure, characterization, and catalytic properties of TNU-9, J. Am. Chem. Soc., 129 (2007) 10870-10885.

[109] J. Shin, S.B. Hong, N,N,N,N ',N ',N '-hexamethylpentanediammonium-MWW layered precursor: A reaction intermediate in the synthesis of zeolites TNU-9 and EU-1, Micropor. Mesopor. Mater., 124 (2009) 227-231.

[110] S. Teketel, U. Olsbye, K.P. Lillerud, P. Beato, S. Svelle, Selectivity control through fundamental mechanistic insight in the conversion of methanol to hydrocarbons over zeolites, Micropor. Mesopor. Mater., 136 (2010) 33-41.

[111] S. Teketel, S. Svelle, K.P. Lillerud, U. Olsbye, Shape-Selective Conversion of Methanol to Hydrocarbons Over 10-Ring Unidirectional-Channel Acidic H-ZSM-22, Chemcatchem, 1 (2009) 7881.

[112] D. Masih, T. Kobayashi, T. Baba, Hydrothermal synthesis of pure ZSM-22 under mild conditions, Chem Commun, (2007) 3303-3305.

[113] S. Teketel, Studies of the Methanol to-hydrocarbon (MTH) reaction over new 8- and 10ring acidic zeolites, 2009, Ph.D. Thesis, University of Oslo.

[114] Pore Size Distribution - Mesopore and Macropore Analysis, 2013

[115] I. Chorkendorff, J.W. Niemantsverdriet, Concepts of Modern Catalysis and Kinetics, 2. ed., Wiley-VCH, Weinheim, 2007.

[116] A. Maczek, Statistical Thermodynamics, 1. ed., Oxford University Press, 2008.

[117] A. Jentys, J.A. Lercher, Techniques of zeolite characterization, in: H. van Bekkum, E.M.

Flanigan, P.A. Jacobs, J.C. Jansen (Eds.) Introduction to zeolite science and practice, Elsevier,

Amsterdam, 2001, pp. 345-386.

[118] A. Zecchina, G. Spoto, S. Bordiga, Probing the acid sites in confined spaces of microporous materials by vibrational spectroscopy, Phys. Chem. Chem. Phys., 7 (2005) 1627-1642.

[119] Raman Spectroscopy, http://cambridgeforecast.wordpress.com/2006/10/11/ramanspectroscopy/, 2006.

[120] P.P. KnopsGerrits, D.E. DeVos, E.J.P. Feijen, P.A. Jacobs, Raman spectroscopy on zeolites, Microporous Mater, 8 (1997) 3-17.

[121] P. Beato, E. Schachtl, K. Barbera, F. Bonino, S. Bordiga, Operando Raman spectroscopy applying novel fluidized bed micro-reactor technology, Catal Today, 205 (2013) 128-133.

[122] P.O. Rønning, Use of Isotopic Labelling in the Study of Methanol Conversion to Hydrocarbons over Zeolite H-ZSM-5, 1998, Ph.D. Thesis, University of Oslo.

[123] E. Benazzi, J.L. Guth, IM-5 zeolites, a process for its preparation and catalytic applications thereof, 2000. 
[124] E. Benazzi, J.-L. Guth, L. Rouleau, Preparation of IM-5 zeolite and its catalytic applications, 1998.

[125] B.C. Wang, Z.J. Tian, P. Li, L. Wang, Y.P. Xu, W. Qu, Y.L. He, H.J. Ma, Z.S. Xu, L.W. Lin, A novel approach to synthesize ZSM-23 zeolite involving $\mathrm{N}, \mathrm{N}$-dimethylformamide, Micropor. Mesopor. Mater., 134 (2010) 203-209.

[126] L.D. Rollmann, J.L. Schlenker, S.L. Lawton, C.L. Kennedy, G.J. Kennedy, D.J. Doren, On the role of small amines in zeolite synthesis, J Phys Chem B, 103 (1999) 7175-7183.

[127] L.D. Rollmann, J.L. Schlenker, C.L. Kennedy, G.J. Kennedy, D.J. Doren, On the role of small amines in zeolite synthesis. 2, J Phys Chem B, 104 (2000) 721-726.

[128] S.H. Lee, C.H. Shin, G.J. Choi, T.J. Park, I.S. Nam, B. Han, S.B. Hong, Zeolite synthesis in the presence of flexible diquaternary alkylammonium ions

$(\mathrm{C}(2) \mathrm{H}(5))(3) \mathrm{N}(+)(\mathrm{CH}(2))(\mathrm{n}) \mathrm{N}(+)(\mathrm{C}(2) \mathrm{H}(5))(3)$ with $\mathrm{n}=3-10$ as structure-directing agents, Micropor. Mesopor. Mater., 60 (2003) 237-249.

[129] N.Y. Chen, W.E. Garwood, Some Catalytic Properties of ZSM-5, a New Shape Selective Zeolite, J Catal, 52 (1978) 453-458.

[130] H. Schulz, "Coking" of zeolites during methanol conversion: Basic reactions of the MTO-, MTP- and MTG processes, Catal Today, 154 (2010) 183-194.

[131] F. Bleken, W. Skistad, K. Barbera, M. Kustova, S. Bordiga, P. Beato, K.P. Lillerud, S. Svelle, U. Olsbye, Conversion of methanol over 10-ring zeolites with differing volumes at channel intersections: comparison of TNU-9, IM-5, ZSM-11 and ZSM-5, Phys. Chem. Chem. Phys., 13 (2011) 2539-2549.

[132] A.J.M. De Man, R.A. Van Santen, The Relation between Zeolite Framework Structure and Vibrational-Spectra, Zeolites, 12 (1992) 269-279.

[133] P.K. Dutta, M. Puri, Synthesis and Structure of Zeolite ZSM-5 - a Raman-Spectroscopic Study, J Phys Chem-Us, 91 (1987) 4329-4333.

[134] A.C. Ferrari, J. Robertson, Interpretation of Raman spectra of disordered and amorphous carbon, Phys Rev B, 61 (2000) 14095-14107.

[135] A.C. Ferrari, J. Robertson, Resonant Raman spectroscopy of disordered, amorphous, and diamondlike carbon, Phys Rev B, 64 (2001).

[136] M. Yoshikawa, G. Katagiri, H. Ishida, A. Ishitani, T. Akamatsu, Resonant Raman-Scattering of Diamondlike Amorphous-Carbon Films, Appl Phys Lett, 52 (1988) 1639-1641.

[137] M. Nakamizo, Kammerec.R, P.L. Walker, Laser Raman Studies on Carbons, Carbon, 12 (1974) 259-267. 


\section{Paper I}

Shape Selectivity in the Conversion of Methanol to Hydrocarbons: The Catalytic Performance of OneDimensional 10-Ring Zeolites: ZSM-22, ZSM-23, ZSM-48, and EU-1. S. Teketel, W. Skistad, S. Benard, U. Olsbye, K.P. Lillerud, P. Beato \& S. Svelle 2. ACS Catalysis (2012) s 26- 37 



\section{Paper II}

Methanol Conversion to Hydrocarbons (MTH) Over H-ITQ-13 (ITH) Zeolite

W. Skistad, S. Teketel, F. Lønstad Bleken, P. Beato, S. Bordiga, M. H. Nilsen, U. Olsbye, S. Svelle \& K. P. Lillerud. Topics in Catalysis (2013) 



\section{Paper III}

Conversion of methanol over 10-ring zeolites with differing volumes at channel intersections: comparison of TNU-9, IM-5, ZSM-11 and ZSM-5. F. Bleken, W. Skistad, K. Barbera, M. Kustova, S. Bordiga , P. Beato, K. P. Lillerud, S. Svelle and U. Olsbye. PCCP 13 (2011) 2539-2549. 



\title{
Conversion of methanol over 10-ring zeolites with differing volumes at channel intersections: comparison of TNU-9, IM-5, ZSM-11 and ZSM-5 $†$
}

\author{
Francesca Bleken, ${ }^{a}$ Wegard Skistad, ${ }^{a}$ Katia Barbera, ${ }^{b}$ Marina Kustova, ${ }^{c}$ \\ Silvia Bordiga, ${ }^{b}$ Pablo Beato,${ }^{c}$ Karl Petter Lillerud, ${ }^{a}$ Stian Svelle ${ }^{a}$ and \\ Unni Olsbye* $* a$
}

Received 30th September 2010, Accepted 11th November 2010

DOI: $10.1039 / \mathrm{c0cp01982h}$

Four 3D 10-ring zeolites, IM-5, TNU-9, ZSM-11 and ZSM-5, with Si/Al = 14-24 and crystal sizes below 2 microns, were tested as catalysts for the methanol to hydrocarbons reaction (MTH) at atmospheric pressure, $350{ }^{\circ} \mathrm{C}$ and WHSV $=9 \mathrm{~h}^{-1}$. All catalysts gave initially full methanol conversion, and showed strikingly similar effluent product selectivities. However, their life-time duration differed significantly, and decreased in the order: ZSM-11 > ZSM-5 $\gg$ TNU-9 > IM-5. A main difference between the two groups of stability behaviour was the size of cavities formed by channel intersections; larger cavities in TNU-9 and IM-5 leading to polyaromatics formation and a more rapid deactivation compared to ZSM-5 and ZSM-11. Effluent yield-conversion plots suggested that polymethylated benzene intermediates were more important in IM-5 and TNU-9 than in ZSM-5 and ZSM-11, where alkene methylation and cracking reactions seemed to dominate product formation. However, this difference had only minor influence on effluent selectivity.

\section{Introduction}

Catalyst design for optimizing reaction selectivity and stability is a major task in state-of-the-art catalysis research. The methanol-to-hydrocarbons (MTH) process, performed with zeolitic catalysts, is a complicated process in which a variety of elementary steps form an intricate interplay: compounds are methylated and dealkylated to form the final products, and this makes it an extremely challenging and interesting catalytic system from the academic point of view. Also, since its first discovery in the 1970s by Chang and Silvestri at Mobil Central Research $^{1}$ it has received considerable industrial interest. ${ }^{2}$ Examples of MTH processes ready for commercialization are the Mobil Oil methanol to gasoline (MTG) process, ${ }^{3}$ the Topsøe integrated gasoline synthesis (TIGAS) process, ${ }^{4}$ the Lurgi methanol to propene (MTP) process $^{5}$ and Norsk Hydro/UOP's methanol to olefins (MTO) process, ${ }^{6}$ all of which use ZSM-5 (MFI) as catalyst except for the last which employs a catalyst based on SAPO-34 (CHA).

\footnotetext{
${ }^{a}$ inGAP Center of Research Based Innovation/Center for Materials

Science and Nanotechnology (SMN), University of Oslo,

Department of Chemistry, N-0315 Oslo, Norway.

E-mail: unni.olsbye@kjemi.uio.no; Tel: + 4722855457

${ }^{b}$ Dipartimento di Chimica IFM-NIS Centre of Excellence,

Università di Torino, Via P. Giuria 7, 10125 Turin, Italy

${ }^{c}$ Haldor Topsøe, Nymollevej 55, DK-2800 Kgs. Lyngby, Denmark

$\dagger$ Electronic supplementary information (ESI) available: XRD, SEM

pictures and product selectivities versus time on stream. See DOI: $10.1039 / \mathrm{c} 0 \mathrm{cp} 01982 \mathrm{~h}$
}

Mechanistically, Chang and Silvestri observed, by contact time variation experiments over a ZSM-5 catalyst at $371{ }^{\circ} \mathrm{C}$, that the MTH reaction proceeded by initial equilibration between methanol and dimethyl ether, followed by light alkene formation and finally aromatics and alkane formation. ${ }^{1}$ Later, two major hypotheses have been made for the MTH reaction mechanism; the autocatalytic mechanism and the hydrocarbon pool mechanism.

The autocatalytic mechanism, suggested by Chen and Reagan in 1979, was based on a conversion versus contact time plot of Chang and Silvestri's original results, in which a steep increase in the slope of the conversion versus contact time plot was observed for conversions above 2\%. ${ }^{7}$ Dessau and Lapierre later suggested that alkenes are the autocatalytic species, and that the MTH reaction consists of a series of alkene methylation reactions, followed by cracking to lighter alkenes. ${ }^{8}$ More than 10 years later, Dahl and Kolboe suggested the hydrocarbon-pool (HP) mechanism, which states that methylation occurs on a reactive organic species adsorbed on the zeolite active site, followed by elimination of products regenerating the initial organic adsorbate. $^{9-12}$ The working catalyst may thus be viewed as an inorganic-organic hybrid catalyst. Kolboe first proposed that this organic adsorbate is a carbenium ion, ${ }^{12}$ but later work from the same group has shown that aromatic molecules, especially polymethylated benzenes, are the dominant hydrocarbon pool species in the MTH reaction over CHA and BEA topologies. ${ }^{11-16}$

There are two major differences between the autocatalytic and the hydrocarbon pool mechanisms: first, the autocatalytic mechanism considers aromatic species solely as reaction 
products and coke precursors, whereas the hydrocarbon pool mechanism considers methyl aromatic species (polymethylated benzenes in particular) as precursors even for alkene formation, and thus as important reaction intermediates. Second, while the autocatalytic mechanism is based on successive reactions between reactants and products of reaction, the hydrocarbon pool mechanism considers reactions between the reactant and trapped reaction products.

There is mounting evidence that catalyst topology influences the reaction mechanism: in SAPO-34 and the corresponding zeolite SSZ-13, which have cavity-type CHA topology, pentaand hexamethyl benzene are the most active aromatic intermediates and the observation of an induction period is a clear indication of the hydrocarbon pool mechanism being in operation. ${ }^{13-15}$ In the large-pore zeolite Beta, Bjørgen et al. showed that hexamethyl benzene is the most active intermediate species together with the heptamethyl benzenium ion, and that the activity of polymethylated aromatics decreases with a decreasing number of methyl groups. ${ }^{16,17}$ Bjørgen et al. also showed that a minimum of four methyl groups are required on the aromatic benzene ring for protonation of the compound in zeolite Beta, and that increasing the number of methyl groups facilitates protonation. ${ }^{18}$ In contrast, the medium-pore sized ZSM-5 (MFI) shows a reverse trend, with the less methylated aromatics being more active. ${ }^{17}$ Also, a comparison of ZSM-5 and SAPO-34 showed that severely deactivated ZSM-5 contained small amounts of tetra-, penta- and hexamethyl benzenes while SAPO-34 contained large amounts of all methylated benzenes as well as several naphthalenes, phenanthrene and pyrene. ${ }^{19}$ This suggests that steric constraints, due to smaller channels and channel intersections, hinder the formation of polyaromatics in ZSM-5.

More recently, Bjørgen et al. studied ZSM-5, and found strong evidence for a dual cycle mechanism. One cycle functions via methylation and dealkylation of aromatic intermediates while the other is a methylation and cracking cycle of linear and branched alkenes. ${ }^{17,19,20}$ Indeed, another study by Teketel et al. on the one-dimensional 10-ring zeolite ZSM-22 (TON) showed little evidence for active aromatic compounds in either products or retained material, and the product spectrum was typical of an alkene methylation/ cracking mechanism. ${ }^{21,22}$ This result indicates that in a 10-ring zeolite, channel intersections are required in order for methylated benzenes to be active intermediates in the hydrocarbon pool mechanism.
The product spectra in 10-ring topologies with different dimensionality are examples of transition state selectivity, i.e.; the possible presence of channel intersections determines which catalytic cycle is more active, and thus also which products are formed (aromatics-rich versus aromatics-poor product spectrum). However, product shape selectivity is also a major factor, as may be seen from the extremely different product spectra in SAPO-34/SSZ-13 and Beta. The main products from the CHA-structure are ethene and propene, ${ }^{15,23}$ while Beta has a product spectrum shifted towards heavier compounds compared to ZSM-5 (gasoline range products). ${ }^{20}$ Comparisons of the products (effluent and retained material) of the MTH reaction over zeolites with similar channel dimensions and differing channel intersections may thus give valuable insight into what volumes and intersection shapes are necessary to accommodate hydrocarbon pool intermediates.

\subsection{Zeolites studied in this work}

In this study, four 3D 10-ring topologies were compared; the archetype MTG catalyst ZSM-5 (MFI topology), which has channel diameter $5.1 \times 5.5 \AA$ along [100] direction, and $5.3 \times 5.6 \AA$ in the [010] direction, and where 3D channel intersections are created at the interface between straight and sinusoidal channels (Fig. 1, right). ZSM-11 (MEL topology, which has channels with diameter $5.3 \times 5.4 \AA$ in two directions, and 3 -dimensionality is created at the interface between two perpendicular, slightly shifted straight channels (Fig. 1, middle right). TNU-9 (TUN topology) consists of two differently sized channel systems with channel dimensions $6.0 \times 5.2 \AA$ and $5.4 \times 5.5 \AA$, respectively. The smaller channels have full $2 \mathrm{D}$ connectivity and the bigger channels provide connection between these 2-dimensional sheets, thus forming a 3D channel system. ${ }^{24}$ The TUN topology forms large, pocketlike, intersections (Fig. 1, middle left). The fourth topology, IM-5 (IMF) is the only structure with only limited 3D connectivity consisting of two $2 \mathrm{D}$ channel systems. One $2 \mathrm{D}$ system has channel diameters $5.5 \times 5.6 \AA$ and $5.3 \times 5.4 \AA$, respectively, while the other $2 \mathrm{D}$ system has channel diameters $4.8 \times 5.4 \AA$ and $5.1 \times 5.3 \AA$, respectively, and a channel with diameter $5.3 \times 5.9 \AA$ is connecting these two $2 \mathrm{D}$ systems (Fig. 1, left). However, this last channel is shifted compared to the intersections in the former $2 \mathrm{D}$ channel system, meaning that the 3-dimensionality increases the intersection space for the smallest 2D channel system only. Slabs of three 2-dimensional channel systems are separated by dense framework, thus
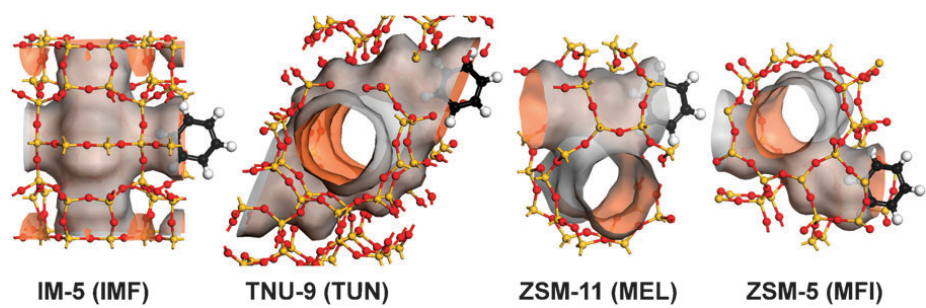

ZSM-11 (MEL)

ZSM-5 (MFI)

Fig. 1 Channel intersections for the four topologies employed in this study. Benzene is placed close to the channel mouth for easier size comparisons. 
forming limited 3-dimensionality. ${ }^{25}$ These four catalysts thus have very similar channel dimensions, whereas the channel intersections give raise to a variety of volumes in which reactions may proceed, as illustrated in Fig. 1. TNU-9 has the biggest cavity associated with a channel intersection, while that of ZSM-5 is the smallest. The cavities are formed in the cross section between two 10-rings in all topologies, however in TNU-9 one of the 10 ring channels expands into a 12-ring in the intersection, thus forming a larger cavity. In ZSM-5 all intersections are equivalent, while in ZSM-11 there are two types of intersections, one leading to cavities of the same size as in ZSM-5 and another giving cavities that are 30\% larger. ${ }^{26}$ IM-5 has three channel intersections. In the largest intersection one of the channels is slightly zig-zaged with alternating 6-ring and 8-ring walls, thus forming a side-pocket in the cavity.

\subsection{Previous work on the various zeolites}

ZSM-5 is by far the most studied catalyst for the conversion of methanol, as well as for a variety of other catalytic systems (see e.g. ${ }^{27}$ and refs therein). Cui et al. reported a study on the conversion of methanol comparing the four catalysts ZSM-11 (MEL), SAPO-34 (CHA), SAPO-5 (AFI) and ZSM-22 (TON). ${ }^{28}$ They reported high activity for the conversion of methanol over ZSM-11 and SAPO-34 with a product spectrum shifted towards heavier compounds for the former. ZSM-11 has been compared to ZSM-5 as catalyst mostly for other reactions than the conversion of methanol, for instance conversion of light paraffins, light olefins and isopentane to aromatic hydrocarbons, ${ }^{29-31}$ the cracking of pentenes to light olefins, ${ }^{32}$ and $p$-xylene alkylation with isopropyl alcohol. ${ }^{33}$ All these reports show that ZSM-5 and ZSM-11 are very similar catalysts, although they do not compare life-times and deactivation curves. Derouane et al. compared ZSM-5 and ZSM-11 for the conversion of methanol under similar conditions as those presented here as early as $1981{ }^{26}$ They reported that both materials were stable catalysts, with $70 \%$ of the products being aliphatics and $30 \%$ aromatics. However, the internal distribution of products within each fraction showed considerable differences, especially for the $C_{1}-C_{2}$ and $C_{8}$ aliphatics. ZSM-5 produced around 7 times more $C_{1}-C_{2}$ than ZSM-11, and ZSM-11 gave 5 times more $\mathrm{C}_{8}$ aliphatics compared to ZSM-5. Within the aromatic fraction, ZSM-11 favoured a heavier product distribution than ZSM-5, with $80 \%$ aromatics with 9 and $10 \mathrm{C}$-atoms as opposed to $26 \%$ in ZSM-5. In ZSM- $565 \%$ of the aromatics were xylenes and ethyl benzenes, compared to only $16 \%$ in ZSM-11. Also, there were distinct differences in the isomer distributions of the various aromatic compounds. ZSM-11 produced much more $o$-xylene and ethyl benzene than ZSM-5, and among the $\mathrm{C}_{9}$ aromatics $80 \%$ were $m+p$-ethyl toluene in ZSM-5, and 1,2,4 trimethyl benzene in ZSM-11. Furthermore, in the same paper, they showed that ZSM-5 is a rather poor catalyst for the alkylation of $p$-xylene when compared to ZSM-11. Methanol conversion over ZSM-5 and ZSM-11 was also compared by Harrison et $a l^{34}$ and they found that both catalysts were very stable, with only minor decay after 24 hours on stream at a WHSV of 1 at $400{ }^{\circ} \mathrm{C}$. After 10 minutes on stream the total amount of aromatics was $40 \%$ in both structures, but their distributions were slightly different. The amount of trimethyl benzene was doubled in ZSM-11 compared to ZSM-5, while
ZSM-5 contained correspondingly more xylenes and toluene. Also, Li et al. showed that methanol conversion over ZSM-5/ZSM-11 intergrowth zeolites and ZSM-5 was almost identical. $^{35}$

TNU-9 and IM-5 are very complex structures that were resolved only recently. ${ }^{24,25,36,37}$ Since the topologies were difficult to assess with crystallographic methods, the structures were tested and compared to known zeolite topologies for various isomerisation and cracking reactions in order to assess their dimensionality. ${ }^{38-41}$ IM-5 was compared to ZSM-5 among a variety of other zeolites for the conversion of methanol, the product spectrum of the aromatic fraction was shifted towards heavier compounds in IM-5 compared to ZSM-5. ${ }^{40}$

\subsection{Method of analysis}

In this study dimethyl ether (DME), which is a condensation product of methanol, and methanol are considered reactants. Hence, conversion is defined as

$$
\begin{gathered}
X_{\mathrm{i}}=[\mathrm{C} \text { in non-oxygen containing compounds } / \\
\mathrm{C} \text { in all compounds }] \times 100 \%
\end{gathered}
$$

The distribution of products for the four catalysts is represented in two ways, as product selectivity with time on stream (in ESI $\dagger$ ) and product yield as a function of conversion. The yield of product $\mathrm{i}\left(Y_{\mathrm{i}}\right)$ is given as

$$
Y_{\mathrm{i}}=\left[\mathrm{C}_{\mathrm{i}} / \mathrm{C} \text { in all compounds }\right] \times 100 \%
$$

and the product selectivity $\left(S_{\mathrm{i}}\right)$ is given as

$$
S_{\mathrm{i}}=\left[Y_{\mathrm{i}} / X_{\mathrm{i}}\right] \times 100 \%
$$

Plots of product yields versus conversion may give valuable insight in reaction mechanisms. ${ }^{42}$ The yield of primary products will cross the origin when extrapolated to zero conversion, while the yield of secondary products will cross the $x$-axis at more than zero conversion. Instability of products towards further reaction is indicated by a decreasing slope of the yield versus conversion curve with increasing conversion, while the yield of secondary products will have an increasing slope at increasing conversion. Abbot and Wojciekowski suggested that such plots, based on time-averaged product analyses over a deactivating catalyst, were equally significant as those obtained by varying the contact time over fresh catalysts. ${ }^{42}$ They used 1-hexene cracking over ZSM-5 as model reaction. Each point in their graphs was obtained by feeding a fixed amount of reactant over a fixed amount of catalyst, and varying total conversion by varying the feed rate. A series of such curves with constant catalyst/feed ratio was obtained, and an optimum performance envelope (OPE) was constructed from them. Analysis of the OPE enabled identifying primary and secondary products in the reaction, as well as determining if the products were stable or not. ${ }^{42}$ Later, Chen et al. used a similar approach for studying ethene oligomerisation over ZSM-5, but used instantaneous yield data. ${ }^{43}$ They reported that yield-conversion plots obtained over a deactivating catalyst overlapped with those obtained over the fresh catalyst at varying contact time, and concluded that catalyst deactivation could in that case be regarded simply as a loss of active catalyst, or a decrease in contact time. Recently, Janssens described "catalyst deactivation as a loss of active 
catalyst" 44 when studying the title reaction over a ZSM-5 catalyst, based on the close correspondence between selectivity changes over a deactivating ZSM-5 catalyst and those reported with changing contact time by Chang and Silvestri in 1977. ${ }^{1}$ Very recently, we obtained results confirming that an instantaneous yield versus conversion plot, obtained for a single catalytic test with deactivation, may be employed similarly in the conversion of methanol over ZSM-5 [to be submitted]. Due to the similarity between the four topologies studied in this work, we hypothesize that deactivation may be regarded as a loss of contact time for all four catalysts, and will employ product yield versus conversion plots obtained during catalyst deactivation as a means of elucidating possible mechanistic differences between the catalysts.

\section{Experimental procedure}

\subsection{Synthesis of materials}

The preparation of TNU-9 was based on the synthesis procedure reported by Hong et al. ${ }^{45}$ where 1-methylpyrrolidine (1-MP) and 1,4-dibromobutane (1,4-DBB) were used as structure directing agents (SDA). Amorphous silica (Aerosil 200, Degussa), $\mathrm{Al}\left(\mathrm{NO}_{3}\right)_{3} \cdot 9 \mathrm{H}_{2} \mathrm{O}(98 \%$, Fluka) and $\mathrm{NaOH}$ (Merck) were used as reagents in the synthesis mixture with the following molar composition: $30 \mathrm{SiO}_{2}: 0.6 \mathrm{Al}_{2} \mathrm{O}_{3}: 10.95 \mathrm{Na}_{2} \mathrm{O}$ : $13.5(1-\mathrm{MP}): 4.5(1,4-\mathrm{DBB}): 1200 \mathrm{H}_{2} \mathrm{O}$. The required amount of 1-MP and 1,4-DBB were mixed with water and stirred until a homogeneous distribution was obtained. The aluminium source and a solution of $50 \mathrm{wt} \% \mathrm{NaOH}$ in water were added consecutively while stirring and the mixture was kept under stirring until a homogeneous solution was obtained. The final solution was added to the silica source and manually stirred for five minutes without further ageing. The final synthesis mixture was charged into $45 \mathrm{ml}$ autoclaves and the crystallization was performed under vertical rotation $(24 \mathrm{rpm})$ at $160{ }^{\circ} \mathrm{C}$ for 13 days. The final product was quenched, recovered by centrifugation and repeatedly washed with water before drying over night.

IM-5 was prepared as stated in ref. 46 by using 1,5-bis( $N$-methylpyrrolidinium)pentane (1,5-MPP) as a SDA. The SDA was prepared by refluxing 1,5-dibromopentane (1,5-DBP) with $1-\mathrm{MP}$ in acetone over night. The product was recovered by filtration and washed several times with acetone before drying and storing in a desiccator prior to use. Amorphous silica (Aerosil 200), $\mathrm{Al}\left(\mathrm{NO}_{3}\right)_{3} \cdot 9 \mathrm{H}_{2} \mathrm{O}(98 \%$, Fluka) and $\mathrm{NaOH}$ (Merck) were used as reagents in the synthesis mixture with a composition as follows: $30 \mathrm{SiO}_{2}: 0.43 \mathrm{Al}_{2} \mathrm{O}_{3}: 10.95 \mathrm{Na}_{2} \mathrm{O}$ : $3.0(1,5-\mathrm{MPP}): 1200 \mathrm{H}_{2} \mathrm{O}$. SDA was dissolved in water and stirred to get a homogeneous mixture. The aluminium source was then added to the solution of the SDA while stirring. After addition of a $50 \mathrm{wt} \%$ solution of $\mathrm{NaOH}$ the synthesis mixture was homogenized and added to the silica source while manually stirred for five minutes without further ageing. The final synthesis mixture was charged into $45 \mathrm{ml}$ autoclaves and crystallized at $160{ }^{\circ} \mathrm{C}$ for 14 days under vertical rotating conditions $(24 \mathrm{rpm})$. The autoclaves were quenched and the final product was recovered by centrifugation and washed several times with water before drying over night.
The as-synthesized samples of TNU-9 and IM-5 were calcined at $550{ }^{\circ} \mathrm{C}$ in a $50: 50$ mixture of $\mathrm{N}_{2}$ and $\mathrm{O}_{2}$ for $8 \mathrm{~h}$ to remove the organic SDA, where $3 \mathrm{~h}$ of the total of $8 \mathrm{~h}$ were spent heating the samples to $550{ }^{\circ} \mathrm{C}$. The calcined samples were ion exchanged three times in $1 \mathrm{M} \mathrm{NH}_{4} \mathrm{NO}_{3}$ solutions for $2 \mathrm{~h}$ at $70{ }^{\circ} \mathrm{C}$ and again calcined at $550{ }^{\circ} \mathrm{C}$ in $50: 50 \mathrm{~N}_{2}: \mathrm{O}_{2}$ for $4 \mathrm{~h}$ to obtain the proton form.

The zeolite Na-ZSM-11 was prepared by the following procedure. A clear solution of sodium aluminate and sodium hydroxide in water was added to tetrabutylammonium hydroxide (TBAOH, $40 \mathrm{wt} \%$ ). Then, tetraethylorthosilicate (TEOS) was slowly added, and the mixture was left with stirring for $2 \mathrm{~h}$ to obtain a homogeneous gel. The composition of the resulting synthesis gel was $1 \mathrm{Al}_{2} \mathrm{O}_{3}: 100 \mathrm{SiO}_{2}: 20 \mathrm{TBA}_{2} \mathrm{O}: 4 \mathrm{Na}_{2} \mathrm{O}$ : $200 \mathrm{H}_{2} \mathrm{O}$. After aging for $2 \mathrm{~h}$ at room temperature, the gel was introduced into a stainless steel autoclave, heated to $170{ }^{\circ} \mathrm{C}$ and kept there for $72 \mathrm{~h}$. Then, the autoclave was cooled to room temperature, the product was suspended in water, filtered by suction, resuspended in water, and filtered again. Finally, the product was dried at $110^{\circ} \mathrm{C}$ for $10 \mathrm{~h}$ and the organic template was removed by controlled combustion in air in a muffle furnace at $550{ }^{\circ} \mathrm{C}$ for $20 \mathrm{~h}$.

A commercial ZSM-5 from Zeochem with $\mathrm{Si} / \mathrm{Al}=12.5$ was used for comparison to a well-known catalyst.

\subsection{Physical characterization of materials}

XRD was employed for confirming the crystal structures. The analyses were performed on a Siemens Bruker D5000 instrument in transmission Debye-Scherrer geometry using $\mathrm{Cu} \mathrm{K} \alpha 1$ radiation. Scanning electron microscopy (SEM) with a FEI Quanta 200 FEG-ESEM equipped with an Everhart-Thornley detector was used for determining particle size distribution and morphology, and EDAX EDS detector for analyzing $\mathrm{Si} / \mathrm{Al}$ ratios. $\mathrm{Si} / \mathrm{Al}$ ratios were also investigated by $\mathrm{NH}_{3}-\mathrm{TPD}$ and electron micro probe (EMP) analyses. EMP was performed on a CAMECA SX 100 instrument. Surface area was determined by BET on a Belsorp-miniII instrument at $-196{ }^{\circ} \mathrm{C}$.

Transmission Infrared (IR) spectroscopy was successfully used to determine acid strength and distribution of acid sites. The spectra were registered with a Nicolet 6700 spectrometer with MCT detector at $2 \mathrm{~cm}^{-1}$ resolution. $\mathrm{CO}$ adsorption with IR was employed for investigation of the nature of acid sites as well as extra framework aluminium in the samples. CO-FTIR at $-196{ }^{\circ} \mathrm{C}$ was performed on self-supporting pellets pre-activated in vacuo and heated to $500{ }^{\circ} \mathrm{C}$ for $1 \mathrm{~h} .80$ torr of $\mathrm{CO}$ was placed in contact with the sample.

\subsection{Catalytic testing}

The materials were pressed into wafers and subsequently crushed and sieved to obtain particles in the range $250-420 \mu \mathrm{m}$. Each catalytic test was performed with $40 \mathrm{mg}$ of catalyst in a fixed bed quartz reactor with $3.0 \mathrm{~mm}$ inner diameter. $30 \mathrm{~mL}$ He was passed through a saturation evaporator containing methanol (BDH Laboratory Supplies, $>99.8 \%$ chemical purity) kept at $20{ }^{\circ} \mathrm{C}$, before being fed to the reactor, giving a WHSV (weight hourly space velocity) of $9.0 \mathrm{~g}$ methanol per g catalyst per hour. The product stream was analyzed with an Agilent 6890 A gas chromatograph with FID detector and 
automatic sampling (Supelco SPB-5 capillary column; $60 \mathrm{~m}$, $0.530 \mathrm{~mm}$ id, stationary phase thickness $3 \mu \mathrm{m}$ ). The pressure of the carrier gas was $42 \mathrm{kPa}$ and the temperature was programmed to $5 \mathrm{~min}$ at $45{ }^{\circ} \mathrm{C}$ before heating $25{ }^{\circ} \mathrm{C} \mathrm{min}{ }^{-1}$ to $260{ }^{\circ} \mathrm{C}$. The catalyst temperature was $350{ }^{\circ} \mathrm{C}$ before onset of methanol for all tests, and once the reaction had started the oven temperature was not adjusted further.

\subsection{Dissolution}

In order to analyze hydrocarbons deposited in the interior channels and cavities of the zeolites during operation, the reaction was stopped by immediate quenching from reaction temperature to room temperature at the end of the experiment. $15 \mathrm{mg}$ of the used catalyst was transferred to a screw-cap Teflon vial and dissolved in $1 \mathrm{~mL} 15 \%$ HF for 1 hour. The organic material was subsequently extracted with $1 \mathrm{~mL}$ $\mathrm{CH}_{2} \mathrm{Cl}_{2}$ (Fluka Puriss) with $\mathrm{C}_{2} \mathrm{Cl}_{6}$ as internal standard. $1 \mu \mathrm{L}$ extract was analyzed with Agilent 6890 N Gas Chromatograph connected to an Agilent 5793 Mass Selective Detector equipped with an HP-5MS column $(60 \mathrm{~m}, 0.25 \mathrm{~mm}$ i.d., stationary phase thickness $0.25 \mu \mathrm{m}$ ). This extraction technique for analyzing trapped hydrocarbons was introduced by Guisnet and co-workers. ${ }^{47}$

\section{Results}

\subsection{Material characterization}

Crystal sizes, BET surface areas and $\mathrm{Si} / \mathrm{Al}$ ratios for the four materials are tabulated in Table 1. SEM pictures and X-ray diffractograms are shown in ESI. $\dagger$ The materials were phasepure, and possessed similar $\mathrm{Si} / \mathrm{Al}$ ratios.

SEM pictures showed that TNU-9 consisted of irregular crystals and agglomerates without a distinct crystal shape. Crystal sizes were in the range $0.2-1 \mu \mathrm{m}$. IM-5 crystals were rod-like with significant intergrowth, but smooth surfaces. Crystal sizes were $0.2-1 \mu \mathrm{m}$. ZSM-5 consisted of crystal agglomerates of varying size and shape, the agglomerate size varying between $0.2-2 \mu \mathrm{m}$. Pictures of ZSM-11 showed uniformly sized agglomerates of spherical shape with rugged surfaces, which indicated that each sphere consisted of smaller agglomerates. The sphere size was in the range $1-2 \mu \mathrm{m}$.

There was good agreement between the methods for measuring acid site density. The acid site density increased in the order ZSM-11 < ZSM-5 < TNU-9 IM-5, and the range of $\mathrm{Si} / \mathrm{Al}$ ratios was 13 to 24 . BET surface areas were slightly smaller than reported previously for TNU-9

Table $1 \mathrm{Si} / \mathrm{Al}$ ratio obtained by microprobe analysis, EDS and TPD, crystal sizes and BET surface area and micropore volume for the four tested catalyst samples

\begin{tabular}{|c|c|c|c|c|}
\hline & TNU-9 & IM-5 & ZSM-11 & ZSM-5 \\
\hline $\mathrm{Si} / \mathrm{Al}$ (micro probe) & 14 & 13 & 24 & 17 \\
\hline $\mathrm{Si} / \mathrm{Al}$ (EDS) & 15 & $14-18$ & 22 & 17 \\
\hline $\mathrm{Si} / \mathrm{Al}$ (TPD) & 17 & 16 & $a$ & 20 \\
\hline Crystal/agglomerate size/microns & $0.2-1$ & $0.2-1$ & $1-2$ & $0.2-2$ \\
\hline BET surface area $/ \mathrm{m}^{2} \mathrm{~g}^{-1}$ & 460 & 340 & 400 & 416 \\
\hline BET micropore vol. $/ \mathrm{cm}^{3} \mathrm{~g}^{-1}$ & 0.191 & 0.141 & 0.170 & 0.152 \\
\hline
\end{tabular}

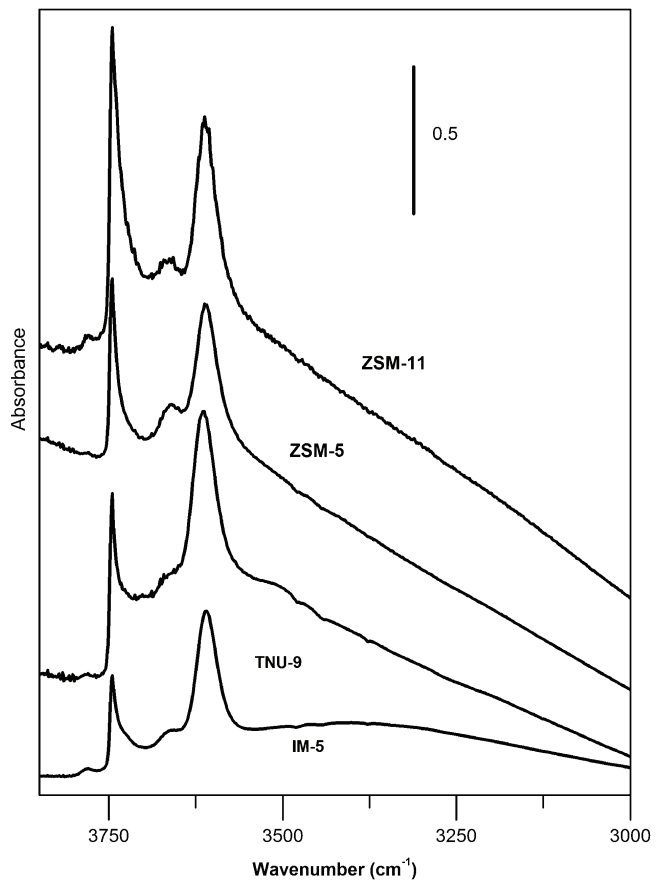

Fig. 2 FTIR spectra obtained on self-supporting pellets activated at $500{ }^{\circ} \mathrm{C}$. Spectra have been normalized at the pellet thickness and area.

and IM-5 with similar Al content. ${ }^{39,48}$ The micropore volume for IM-5 corresponds well with previous results while it is higher for TNU-9 in this study. None of the materials contained mesopores.

Brønsted and Lewis sites were evaluated by IR spectroscopy as reported in Fig. 2. The scattering profile varied in this set of samples (testifying different powder shapes), while all materials presented similar spectroscopic features in terms of number and positions of the bands. In particular all spectra were characterized by two main bands centred at $3745 \mathrm{~cm}^{-1}$ and at $3614 \mathrm{~cm}^{-1}$, assigned to free isolated external silanols and strong Brønsted acid sites, respectively. The band due to silanols clearly showed a tail on the low frequency side (more pronounced in the case of IM-5 and TNU-9). The silanol band was more intense in ZSM-11, followed by ZSM-5 and TNU-9 which were similar, while in IM-5 it was definitely less intense. This result agrees well with the morphology observations from SEM (see above). As expected on the basis of $\mathrm{Al}$ content, the intensity of the band at $3614 \mathrm{~cm}^{-1}$ was very similar in all samples. All samples showed a minor band at $3665 \mathrm{~cm}^{-1}$ due to partial extra-framework Al species (more abundant in the case of ZSM-5) and a small band at $3780 \mathrm{~cm}^{-1}$, ascribed to $\mathrm{OH}$ groups associated to extra-framework Al species (except in the case of ZSM-5). IM-5 and TNU-9 were characterized by broad bands that extended below $3500 \mathrm{~cm}^{-1}$, associated to interactive silanol bands due to internal defects (i.e. silanol nests). These assignments were done after testing the acidities of these species by $\mathrm{CO}$ adsorption at liquid 


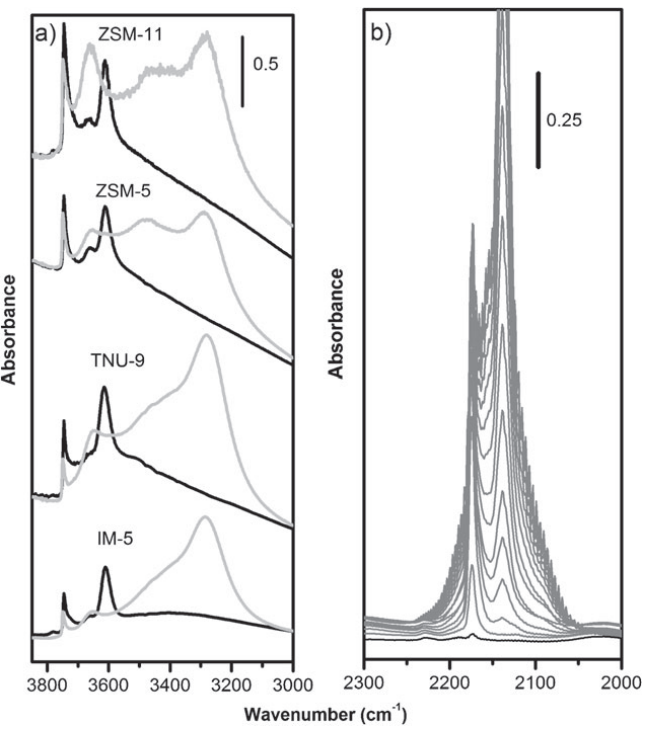

Fig. 3 a) The effect of $\mathrm{CO}$ interaction with all the four catalysts (activated samples, black curves; highest $\mathrm{CO}$ coverage, grey curves). (b) A full set of curves obtained at increasing CO coverages on ZSM-5 sample in the CO stretching region.

nitrogen temperature (Fig. 3a), showing that the acid strengths for the four samples were equivalent; upon $\mathrm{CO}$ adsorption bands associated to silanols were red shifted to $3656 \mathrm{~cm}^{-1}$ $\left(\Delta \nu=+89 \mathrm{~cm}^{-1}\right)$, while the absorption ascribed to partial extra framework Al species, more evident in the case of ZSM-11 and ZSM-5 were red shifted to $3477 \mathrm{~cm}^{-1}\left(\Delta \nu=+189 \mathrm{~cm}^{-1}\right)$. Finally, all samples showed a comparable shift also in the case of the band associated to strong acid sites (red shift to $3286 \mathrm{~cm}^{-1}$ $\left.\left(\Delta \nu=+328 \mathrm{~cm}^{-1}\right)\right)$. The $\mathrm{CO}$ stretching region was very similar in all cases. For this reason part b of Fig. 3 reports the full set of data only for ZSM-5. At low CO coverages a band is observed at $2174 \mathrm{~cm}^{-1}$. This band is assigned to blue shifted $\mathrm{CO}$ stretching due to the formation of adducts with

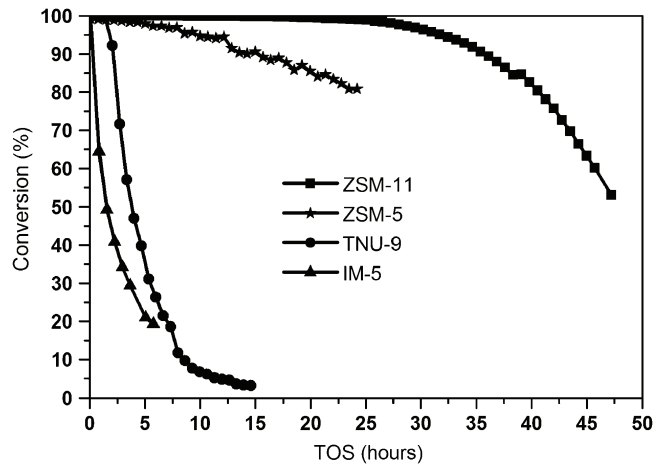

Fig. 4 Conversion of methanol over IM-5, TNU-9, ZSM-11 and ZSM-5 at atmospheric pressure, $350{ }^{\circ} \mathrm{C}$ and WHSV $=9 \mathrm{~h}^{-1}$. strong Brönsted sites. At higher coverages, a component at $2138 \mathrm{~cm}^{-1}$ is due to "liquid-like" CO. The absence of bands around $2220 \mathrm{~cm}^{-1}$ indicates absence of strong Lewis sites.

\subsection{Catalyst stability}

Fig. 4 shows the deactivation curves for the conversion of methanol over the four catalysts. Initially all methanol was converted over all four catalysts, except for ZSM-5 which gave $99 \%$ conversion initially. Under the chosen reaction conditions, methanol breakthrough over TNU-9 was detected after $100 \mathrm{~min}$ on stream. IM-5 deactivated rapidly after the first analysis (5 min on stream). The most long-lived catalyst was ZSM-11, which did not show methanol break-through before 25 hours on stream, and converted more than $50 \%$ of the methanol after 48 hours on stream. In comparison, ZSM-5 started deactivating soon after the onset of methanol and the conversion level decreased very slowly to $80 \%$ after 24 hours on stream.

\subsection{Catalyst selectivity}

The product distributions, represented by gas chromatograms obtained after 5 minutes on stream, are shown in Fig. 5. The product selectivities were strikingly similar for the four topologies. The most prominent peak was $\mathrm{C}_{3}$ in all the catalysts while $\mathrm{C}_{2}$ was the second highest peak in all chromatograms except for TNU-9. The second highest peak in TNU-9 was isobutane which was the third highest for the other three samples. However, the sum of butenes and butanes were higher than $\mathrm{C}_{2}$ in all topologies. Within the aromatics fraction, the two most abundant peaks were $m+p$-xylene and 1,2,4-trimethyl benzene.

\subsection{Product yields}

3.4.1 Yield versus conversion for TNU-9. The product yields as a function of conversion, shown in Fig. 6, may provide an indication about primary versus secondary, and stable versus unstable, products, as explained by Abbot and Wojciechowski. ${ }^{42}$ The onset of reaction is at the right end of the graph ( $100 \%$ conversion) and time evolution goes left from this point. The $\mathrm{C}_{3}$ fraction was most abundant except when the conversion capacity was more than $100 \%$. All product

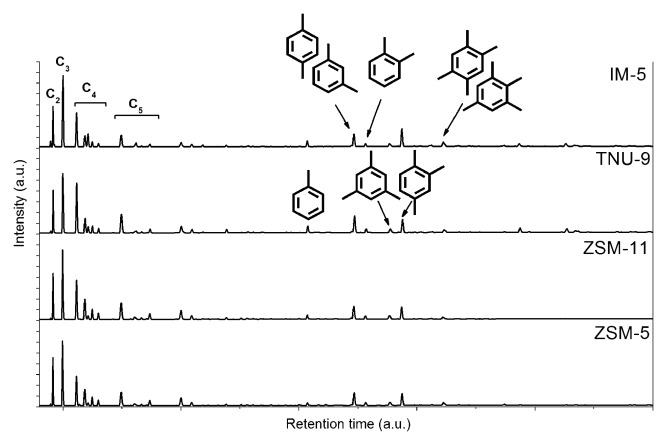

Fig. 5 The chromatograms of the effluent stream after 5 minutes of methanol feed for IM-5, TNU-9, ZSM-11 and ZSM-5 at atmospheric pressure, $350{ }^{\circ} \mathrm{C}$ and WHSV $=9 \mathrm{~h}^{-1}$. 

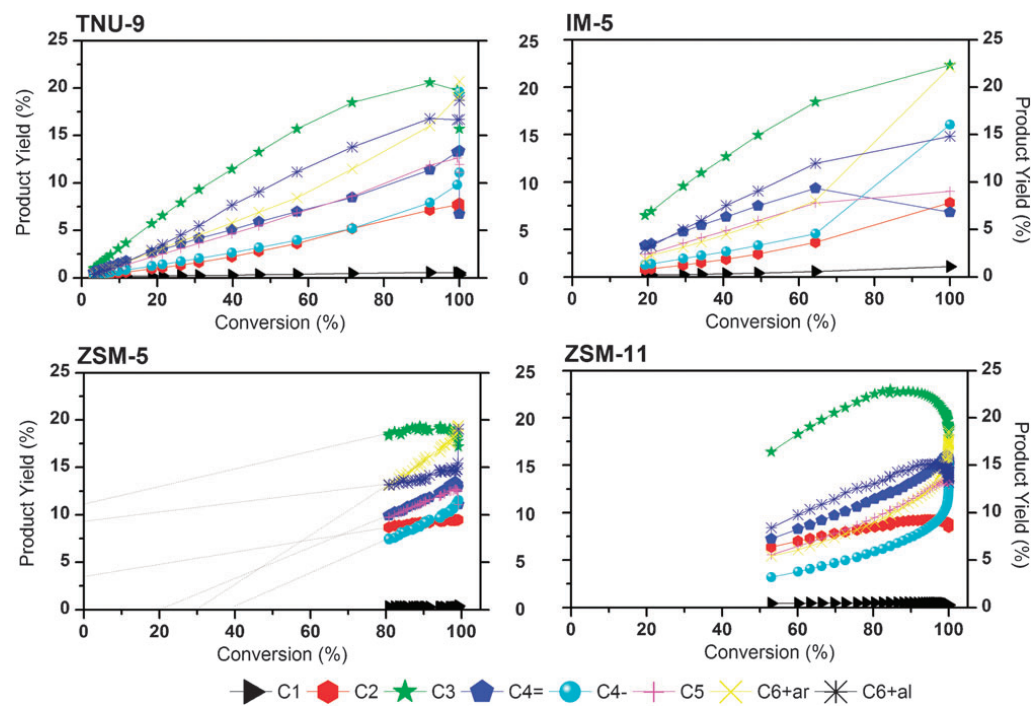

Fig. 6 Product yields as a function of conversion for TNU-9 (top left), IM-5 (top right), ZSM-5 (bottom left) and ZSM-11 (bottom right). Conversion of methanol performed at atmospheric pressure, $350{ }^{\circ} \mathrm{C}$ and WHSV $=9 \mathrm{~h}^{-1}$. The curves are extrapolated to cross the axes in order to determine the stability of the products and if they are formed as secondary products.

yield curves seemed to pass through the origin when extrapolated to zero conversion, suggesting that they are primary products. However, their slope with increasing conversion varied as follows: the slope of the $\mathrm{C}_{3}$ yield curve decreased with increasing conversion, indicating that $\mathrm{C}_{3}$ is an unstable product. The yield of $\mathrm{C}_{3}$ decreased dramatically at full methanol conversion. This observation may suggest that $\mathrm{C}_{3}$ is formed (directly or indirectly) by methylation reactions, but is converted via other reactions (at least in the absence of methanol). The abrupt drop in $\mathrm{C}_{3}$ yield at full conversion could be due to competitive adsorption of methanol (p.a. $\left.=754 \mathrm{~kJ} \mathrm{~mol}^{-1}\right)^{49}$ and propene (p.a. $\left.752 \mathrm{~kJ} \mathrm{~mol}^{-1}\right)^{49}$ on the acid sites, possibly preventing hydrocarbon interconversion reactions in the presence of methanol. Such an effect has previously been reported by Svelle et al. for the oligomerisation of propene in the presence or absence of methanol. ${ }^{50}$ The slope of the $\mathrm{C}_{2}$ yield curve increased with increasing conversion, suggesting that $\mathrm{C}_{2}$ is formed both as a primary and secondary product. A small decrease at full conversion may suggest instability towards further reaction. Both butenes and butanes showed behaviours corresponding to stable primary and secondary products. At full conversion, however, the butene yield dropped significantly, while the slope of the butane yield curve further increased. As for $\mathrm{C}_{3}$, the sudden decrease in butene yield at full conversion could be due to slower formation and faster conversion in the absence of methanol. It should be noted that the butene fraction consisted of iso- and linear butenes. The linear butenes are products of propene methylation while iso-butene is a cracking product. The relative abundance of linear butenes increased with increasing conversion (not shown). At full conversion both fractions were halved. The corresponding increase in butane yield could not fully account for the decrease in butene yields. Within the $\mathrm{C}_{6+}$ fraction the aromatics had increasing slope with increasing conversion, suggesting they are stable primary and secondary products, while aliphatics had increasing then decreasing slope, indicating they are unstable primary and secondary products. The analysis point obtained at full conversion deviated from the general behaviour, giving an abrupt drop in $\mathrm{C}_{6}{ }^{+}$aliphatics and a corresponding increase in the $\mathrm{C}_{6}{ }^{+}$ aromatics yield. Fig. 7, top left panel, shows the product yields for the aromatic fraction. These graphs suggest that the primary products were the most heavily methylated benzenes, while the less methylated benzenes were secondary products. Also, the primary (tetra-,) penta- and hexamethyl benzenes were unstable, while toluene, xylene and in part trimethyl benzenes were stable secondary and primary products. 1,2,4trimethyl benzene seems to be a primary, stable product.

3.4.2 Yield versus conversion for IM-5. Fig. 6, top right panel, shows the product yields as function of conversion for IM-5. For the IM-5 it is important to note that from the first analysis point at $100 \%$ conversion to the second analysis there was a decrease in conversion of $35 \%$. This means that the yield versus conversion curve is less detailed in the area used for determining the stability of the products with respect to further reactions. However, changes in slopes with changing conversion may still yield information about the reaction scheme.

Extrapolation of the points obtained at lowest conversion towards zero conversion indicates that all products were formed from the beginning, and that none of them can be considered purely secondary products. However $\mathrm{C}_{6+}$ aromatics, $\mathrm{C}_{4}$ alkanes and $\mathrm{C}_{2}$ increased more than linearly with conversion, thus indicating secondary product behaviour, in addition to being primary. $\mathrm{C}_{3}, \mathrm{C}_{4}, \mathrm{C}_{5}$ alkenes and $\mathrm{C}_{6}+$ aliphatics have decreasing slopes with increasing conversion, suggesting they 

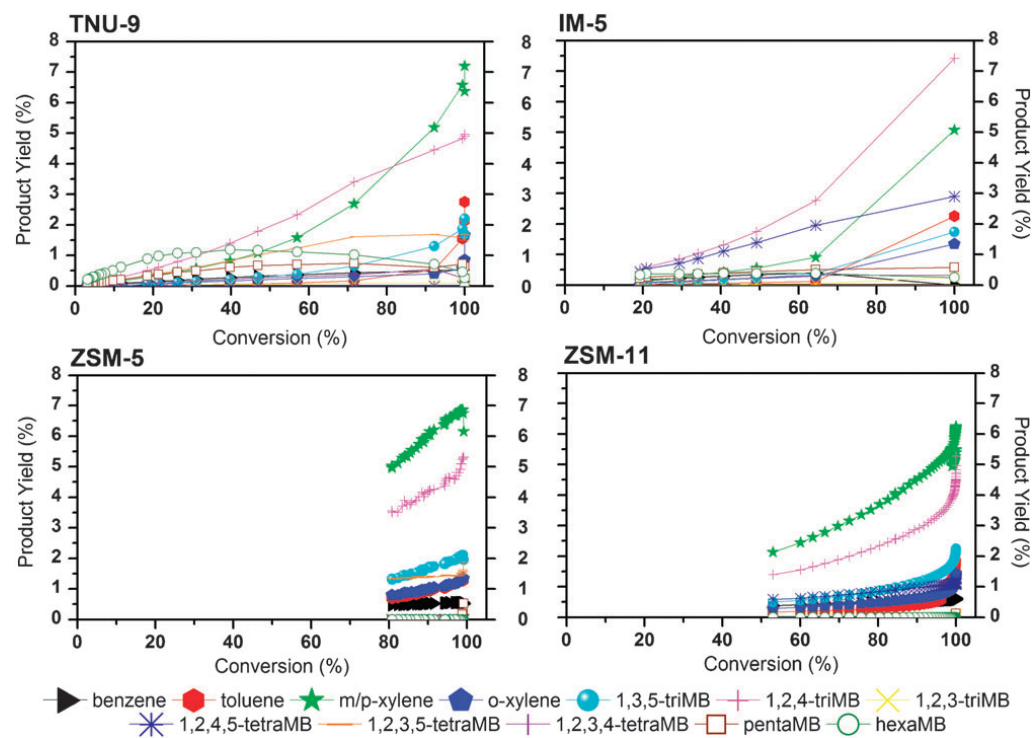

Fig. 7 Product yields of methylated benzenes (MBs) as a function of conversion. Conversion of methanol performed at atmospheric pressure, $350{ }^{\circ} \mathrm{C}$ and WHSV $=9 \mathrm{~h}^{-1}$.

are unstable products. The aromatic yields over IM-5 is presented in Fig. 7 (top right panel). The most abundant product was 1,2,4-trimethyl benzene with increasing slope with higher conversion, while extrapolation towards zero conversion crossed the origin. This indicates a primary and secondary stable product. The curve for $m / p$-xylene also increased with increasing conversion and seemed to cross the abscissa at more than zero conversion, indicating a secondary product. 1,2,4,5-tetramethyl benzene gave a straight line typical for a primary, stable product. Toluene, $o$-xylene, and the trimethyl benzenes had increasing slope with increasing conversion, indicating secondary, stable product behaviour. Penta and hexamethyl benzene were produced in very small amounts, and the yield decreased slightly at high conversion, suggesting that they could be unstable products.

3.4.3 Yield versus conversion for ZSM-5. The product yields as functions of conversion for ZSM-5 are shown in Fig. 6, bottom left panel.

Since ZSM-5 was only tested until it had deactivated to $80 \%$ conversion, the plot does not yield information about primary product formation. However the curvatures at high conversion may indicate whether products were (partly) secondary and whether they were unstable towards further reaction. Linear extrapolation of the curves towards the axes is included as a guide for the eye for ZSM-5 in Fig. 6. When the extrapolated curve crosses the abscissa (conversion axis), it suggests that the product has been formed as a secondary product. Crossing on the ordinate (yield axis) indicates an unstable product.

At less than maximum conversion $\mathrm{C}_{3}$ was the most abundant product, followed by the $\mathrm{C}_{6+}$ aromatics and $\mathrm{C}_{6+}$ aliphatics. The $\mathrm{C}_{3}$ yield decreased at full conversion, suggesting instability towards further reactions. The yield of the $\mathrm{C}_{6+}$ aromatics fraction increased linearly with conversion and became the most abundant product group at the highest conversion. An extrapolation of this line crossed the abscissa at $30 \%$ conversion, indicating secondary product behaviour. Extrapolation of the $\mathrm{C}_{6}+$ aliphatics crossed the ordinate above origin, indicating they are unstable products. The $\mathrm{C}_{4}$ alkene yield was higher than the $\mathrm{C}_{4}$ alkane yield throughout the reaction. Extrapolation of the curves for both butenes and butanes suggests secondary product behaviour. The small decrease in alkene yield when closing in on $100 \%$ conversion indicates that this product was somewhat unstable towards further reactions. The $\mathrm{C}_{5}$ fraction yield was identical to the $\mathrm{C}_{4}$ alkene yield. No curvature was observed for the $\mathrm{C}_{2}$ product yield. The extrapolated line, however, crossed the ordinate, indicating that the $\mathrm{C}_{2}$ fraction was to a small degree unstable.

Yields versus conversion for the methylated benzenes are shown in Fig. 7 (bottom left panel). The most abundant aromatic fraction was $m / p$-xylene, followed by 1,2,4-trimethyl benzene. Extrapolation of both graphs suggested that they are secondary products. Also the curves for $o$-xylene, 1,3,5trimethyl benzene and 1,2,3,5-tetramethyl benzene indicated that they are formed as secondary products, but with a much lower yield. The yield of benzene was constant throughout the reaction, while only traces of penta- and the remaining tetramethyl benzenes were detected, even at maximum conversion. Hexamethyl benzene was not observed.

3.4.4 Yield versus conversion for ZSM-11. Fig. 6, bottom right panel, shows the product yields as functions of conversion for ZSM-11. The material was tested in the range 53 to $100 \%$ conversion, and the plot does therefore not yield information on primary product formation. $\mathrm{C}_{3}$ clearly curved downwards with high conversion suggesting an unstable 


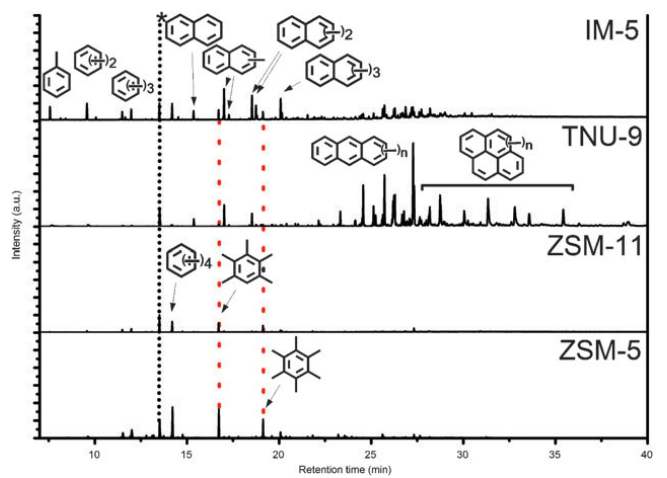

Fig. 8 Chromatogram of retained material at end of experiment for each catalyst. * indicates the internal standard, $\mathrm{C}_{2} \mathrm{C}_{16}$. The red lines are included to help recognition of the penta- and hexamethyl benzene peaks in all of the samples. Three and four ring aromatics are not identified in detail, but their approximate positions are shown in the graph.

product. The same trend, but less clearly, was seen for $\mathrm{C}_{6+}$ aliphatics and $\mathrm{C}_{2}$. The slope of the yields of $\mathrm{C}_{4}$ alkanes and $\mathrm{C}_{6+}$ aromatics increased with increasing conversion, indicating secondary stable products. The $\mathrm{C}_{4}$ alkene yield curve had a slight curvature upwards from 80 to $98 \%$ conversion, and then a downwards curvature at very high conversion indicating that this product was secondary and unstable. Within the aromatic fraction, Fig. 7, all detected products, with the exception of benzene and 1,2,3-trimethyl benzene, had yield curves with increasing slopes as conversion increased, suggesting secondary stable products. Continued increase at $100 \%$ conversion indicated that they are mainly formed by reactions other than methylation reactions. Benzene and 1,2,3-trimethyl benzene had linear curves which extrapolated to the origin, indicating stable products. 1,2,3,4-tetra-, penta- and hexamethyl benzene were not detected.

\subsection{Retained material}

The chromatograms of the retained material in the catalysts at the time when the reaction was stopped are shown in Fig. 8. IM-5 contained substantial amounts of methyl benzenes with 1 to 6 methyl groups, as well as methylated naphthalenes. There were also several small peaks due to polyaromatics with 3 and 4 rings. TNU-9 contained almost no methylated benzenes, but naphthalenes with 0,1 and 2 methyl groups were detected. The biggest and most abundant peaks corresponded to compounds with 3 and 4 aromatic rings. ZSM-5 and ZSM-11 contained almost exclusively benzenes with $3,4,5$ and 6 methyl groups, with tetra- and penta-methyl benzenes being most abundant. More methylated benzenes were present in ZSM-5 compared to ZSM-11.

\section{Discussion}

The aim of the present study was to elucidate the influence of topology, in particular the size of cavities formed by channel intersections, on the $\mathrm{MTH}$ reaction over 3D 10-ring zeolites. For this purpose, a large number of zeolite syntheses were performed, resulting in four materials (among which one was a commercial sample) with different topologies and similar $\mathrm{Si} / \mathrm{Al}$ ratios, crystal sizes and acid strengths.

All four topologies displayed full initial conversion at $350{ }^{\circ} \mathrm{C}$ and WHSV $=9 \mathrm{~h}^{-1}$, but lifetimes varied considerably in the order ZSM-11 > ZSM-5 》 TNU-9 > IM-5 (Fig. 4). Analysis of retained material in the catalysts after the life-time tests showed that TNU-9 and IM-5 contained more retained material than ZSM-5 and ZSM-11 (Fig. 8). Furthermore, TNU-9 contained mainly polycyclic aromatics, while IM-5 contained methylated naphthalenes and benzenes. ZSM-11 and ZSM-5 contained mainly methylated benzene with only traces of naphthalenes. When considering that TNU-9 and IM-5 were tested for much shorter times than ZSM-5 and ZSM-11 before analyzing the retained material, it is tempting to correlate the enhanced deactivation rate of IM-5 and TNU-9 to their selectivity towards polyaromatic compound formation. Such a conclusion would be in line with previous studies of ZSM-5 and SAPO- $34,{ }^{19}$ where it was shown that rapidly deactivating SAPO-34 contained more than an order of magnitude more retained aromatics than the rather stable ZSM-5 after severe deactivation. In that study, topology was suggested as a main reason for the more rapid deactivation of SAPO-34, related both to its 12-ring cavities which allow polycyclic aromatics formation, and its narrow 8-ring pore openings which severely restrict diffusion compared to the 10-ring channels of ZSM-5. In the present work, the studied topologies have very similar channel sizes, while the size of channel intersections varies significantly, in the order: TNU-9 $>$ IM-5 > ZSM-11 > ZSM-5. This order is in general agreement with the higher selectivity towards polyaromatic compounds in TNU-9 and IM-5 compared to ZSM-5 and ZSM-11. However, it does not fully account for the relative stabilities of all four topologies.

From literature it is known that catalyst stability in the MTH reaction is also influenced by acid site density, ${ }^{23,51}$ crystal size ${ }^{52}$ and acid site strength. ${ }^{15}$ From IR measurements the acid site strength was found to be equal for the four topologies (Fig. 3), thus this parameter is not likely to have influenced the variations in catalyst life-time. Concerning acid site density, the $\mathrm{Si} / \mathrm{Al}$ ratios (Table 1) cover a narrow range in the order ZSM-11 > ZSM-5 > TNU-9 > IM-5, which is in the same order as the life-times. Larger crystals have previously been linked to shorter catalyst lifetime due to diffusion limitations. ${ }^{52}$ In this study, the crystal size increased in the order ZSM-11 $<$ ZSM-5 $<$ TNU-9 $<$ IM-5, which is also in agreement with the observed relative stability. In conclusion, several parameters may have contributed to the relative variations in catalyst life-time between the four topologies. However, the clear distinction into two groups of stabilities, with TNU-9 and IM-5 deactivating rapidly and ZSM-5 and ZSM-11 deactivating slowly, only correlates with the differences in formation of polyaromatics within the materials. This result indicates that the cavity size of 3D 10-ring zeolites is of primary importance for their stability as MTH catalysts.

It has previously been suggested that deactivation of ZSM-5 is primarily linked to the formation of external coke, while deactivation in cage-like structures such as SAPO-34 and zeolite beta are more strongly correlated to the build-up of polyaromatics within the crystals. ${ }^{19,52}$ This could mean that the most important source of deactivation is not the same for the four systems studied. However, the close similarity in effluent product distributions among them (Fig. 5-7) indicates 
that formation of deactivating coke does not change the nature of the catalytic sites, but rather removes active catalytic sites from the system, in agreement with the underlying assumption of the yield versus conversion plots in Fig. 6 and 7.

The similarities between the four tested topologies with respect to effluent product selectivities and yield evolution with declining conversion at increasing times on stream (Fig. 5-7) is a clear evidence of the importance of the channel dimensions in the MTH reaction. However, Teketel et al. showed that in a 10-ring structure without channel intersections (TON topology), aromatic products are formed only to a minor extent, and play an insignificant (if any) role in olefins formation reactions. ${ }^{21}$ This result implies that the channel intersections still have profound influence on the MTH reaction. However, the great similarity in the effluent for the four structures investigated in this work, shows that small variations in these intersection volumesas long as they are present - are not overly significant for the effluent product selectivity.

Concerning reaction mechanisms, a comparison between the yield versus conversion curves obtained over the four topologies show very similar patterns: $\mathrm{C}_{3}$ is the main product at less than $100 \%$ methanol conversion, followed by $\mathrm{C}_{6}+$ aliphatics, $\mathrm{C}_{6+}$ aromatics and $\mathrm{C}_{4}$ alkenes. Furthermore, $\mathrm{C}_{3}$ and $\mathrm{C}_{4}$ alkenes as well as $\mathrm{C}_{6}+$ aliphatics seem to be unstable towards further reaction, while $\mathrm{C}_{4}$ alkanes and $\mathrm{C}_{6}$ aromatics are formed by secondary reactions from other products (Fig. 6). None of the $\mathrm{C}_{6}-\mathrm{C}_{10}$ aromatics decrease in yield when methanol conversion approaches full conversion over the four studied topologies, suggesting that $\mathrm{C}_{6}-\mathrm{C}_{10}$ aromatics are not major intermediates in the MTH cycle over these topologies under the conditions studied here. This conclusion is in agreement with previous studies of the title reaction over $\mathrm{ZSM}-5$ with higher $\mathrm{Si} / \mathrm{Al}$ ratio, showing that the alkene-based cycle is the main source of $\mathrm{C}_{3+}$ formation under the conditions used here. ${ }^{17,19,20}$ Also, the yield of the majority of the methylated benzenes (MBs) increases sharply at close to full methanol conversion, especially triMBs (ZSM-11, ZSM-5) and xylenes (TNU-9), indicating that they are formed as secondary products, and mainly from reactions other than methylation reactions. Their yield increase at close to full methanol conversion coincides with an abrupt decrease in $\mathrm{C}_{3}$ yield. This result suggests that propene is converted to aromatic compounds via oligomerisation/cyclisation reactions at full methanol conversion. The simultaneous decrease in butene and increase in butane yields supports this hypothesis, since formation of aromatics is coupled to hydrogen transfer reactions. This observation agrees well with both aromaticsand alkene-based MTH reaction mechanisms.

A main difference between the topologies is observed for the high end of the polyMB fraction (Fig. 7): While only $\mathrm{C}_{6}-\mathrm{C}_{10}$ aromatics are observed in the effluent of ZSM-5 and ZSM-11, also penta- and hexaMB are observed in the effluent of TNU-9 and IM-5. Furthermore, these two compounds go through a yield maximum at $20-70 \%$ methanol conversion over both topologies, suggesting that they are unstable products which are converted by secondary reactions. The decrease in pentaand hexaMB yield over TNU-9 at full methanol conversion suggests that they are converted by reactions other than methylation reactions, possibly dealkylation to form light alkenes, as previously proposed by Bjørgen et al. for Beta zeolite and
Arstad et al. for SAPO-34. ${ }^{13,53}$ These data further suggest that an aromatics-based MTH cycle is more important in TNU-9 and IM-5 compared to ZSM-5 and ZSM-11, where an alkenebased cycle seems to dominate. In the effluent of ZSM-5 and ZSM-11, $m / p$-xylene were the most abundant aromatic products, followed by 1,2,4-trimethyl benzene. In TNU-9 this order was the same at high conversion, but reversed at lower conversion levels. In IM-5 it was reversed over the whole conversion range. This shift towards polymethylated benzenes with a higher number of methyl groups for TNU-9 and IM-5 is in agreement with the hypothesis that the aromatics-based hydrocarbon pool mechanism is more significant in these topologies. Also, the relatively high content of iso-butane in TNU-9 compared to the other structures after 5 minutes on stream (Fig. 5) may be explained by the higher selectivity towards polyaromatics and the greater significance of the aromatic cycle in this topology.

It should be noted that, when considering the pore size of TNU-9 and IM-5, TNU-9 has clearly larger channels than the other topologies. However, IM-5 has only slightly larger channels than ZSM-11 and ZSM-5, and it is therefore surprising to find penta- and hexaMB among the effluent products of this topology. It may be speculated that these large polyMBs are formed and converted on the outer surface of IM-5 (and TNU-9), but it is not obvious why it happens on this topology while not over ZSM-5 and ZSM-11. TNU-9 and IM-5 also differ from ZSM-5 and ZSM-11 in the $\mathrm{C}_{2}$ yield curve. In TNU-9 and IM-5 the slope of the $\mathrm{C}_{2}$ yield curve increases slightly with increasing conversion. Svelle et al. coupled the formation of ethene to the aromatic cycle while all other alkenes were considered products of the alkene cycle. ${ }^{54}$ An increase in $\mathrm{C}_{2}$ yield is therefore in agreement with polymethylated benzene reactions being more important at higher conversion in TNU-9 and IM-5. In ZSM-5 and ZSM-11 the slope of the $\mathrm{C}_{2}$ yield curve decreased with increasing conversion in agreement with the alkene cycle being dominant in these topologies.

Another observation which deserves attention is the behaviour of the four topologies at full methanol conversion. The yield versus conversion plots show very similar product yields at close to full methanol conversion over all catalysts (Fig. 5). At full conversion, significant further reaction of $\mathrm{C}_{3}$ (and $\mathrm{C}_{4}{ }^{=}$to $\mathrm{C}_{4}{ }^{-}$) is observed over TNU-9, ZSM-5 and ZSM-11. Such further conversion is not observed over IM-5, which deactivated significantly between the first and second effluent analysis (Fig. 4). The similarity between IM-5 (first analysis) and the "close-to-100\% methanol conversion" yields for the other three catalysts, may suggest that those three catalysts, TNU-9, ZSM-5 and ZSM-11, have significantly higher initial activity than IM-5, or that IM-5 deactivates extremely fast compared to the three other topologies, even before the first analysis point (5 min on stream). When considering the slope of the conversion versus time on stream curve for TNU-9 versus IM-5 after onset of deactivation (Fig. 4), they are very similar. This result suggests that the deactivation rate of the two catalysts is also similar, and points to a higher initial activity as a main reason for the different initial behaviour of IM-5 compared to the other three topologies (Fig. 5). IM-5 is the only topology among the four studied topologies that has only limited 3D connectivity. Previous studies in our group have shown that one-dimensional ZSM-22 (TON topology) requires significantly longer contact times 
compared to $3 \mathrm{D}$ topologies like MFI and CHA to achieve appreciable conversion. ${ }^{21}$ Thus, one may speculate that a lower initial activity of IM-5 could result from diffusivity restrictions in this limited 3D topology compared to the three 3D topologies, although further studies would be required to elucidate this point in more detail.

Finally, when considering the product yield versus conversion plots, all products have a linear approach towards origin at low conversion for TNU-9, the only topology which was tested until low conversions. This result suggests that all products are formed as primary products, in disagreement with both alkene- and aromatics-based MTH mechanisms. We believe the result is an artifact, due to a significant extent of secondary reactions within the crystals before diffusing into the bulk. Such secondary reactions may also contribute to the product distributions being so similar in the four topologies.

\section{Conclusion}

ZSM-5, TNU-9, IM-5 and ZSM-11 are all active for the conversion of methanol at $350{ }^{\circ} \mathrm{C}$ and WHSV $=9 \mathrm{~h}^{-1}$, with very similar effluent product distributions. However, their selectivities towards the formation of heavy aromatic compounds entrapped within the structures differ significantly. TNU-9 and IM-5 showed a high content of methylated aromatics and poly-aromatics after testing, and the fast deactivation observed in these two structures were related to the formation of such species. Furthermore, the product streams of TNU-9 and IM-5 contained unstable penta- and hexamethyl benzenes, which were not detected for ZSM-11 and ZSM-5. The presence of unstable penta- and hexamethyl benzenes, as well as the substantial selectivity towards polyaromatics, indicates that the aromatic hydrocarbon pool cycle is relatively more important in TNU-9 and IM-5 compared to ZSM-5 and ZSM-11.

\section{References}

1 C. D. Chang and A. J. Silvestri, J. Catal., 1977, 47, 249-259.

2 S. Kvisle, T. Fuglerud, S. Kolboe, U. Olsbye, K. P. Lillerud and B. V. Vora, in Handbook of Heterogeneous Catalysis, ed. H. Ertl, F. Knözinger, F. Schüth and J. Weitkamp, Wiley-VCH, 2008, vol. 6, ch. 13.14, pp. 2950-2965.

3 J. Cobb, in New Zealand Synfuel, ed. G. Connell, Cobb/Horwood Publications, Auckland, New Zealand, 1995, p. 1.

4 J. Topp-Jørgensen, Stud. Surf. Sci. Catal., 1988, 36, 293-305.

5 H. Koempel and W. Liebner, Stud. Surf. Sci. Catal., 2007, 167, 261-267.

6 J. Q. Chen, A. Bozzano, B. Glover, T. Fuglerud and S. Kvisle, Catal. Today, 2005, 106, 103-107.

7 N. Y. Chen and W. J. Reagan, J. Catal., 1979, 59, 123-129.

8 R. M. Dessau and R. B. Lapierre, J. Catal., 1982, 78, 136-141.

9 I. M. Dahl and S. Kolboe, Catal. Lett., 1993, 20, 329-336.

10 I. M. Dahl and S. Kolboe, J. Catal., 1994, 149, 458-464.

11 S. Kolboe, Acta Chem. Scand., Ser. A, 1986, 40a, 711-713.

12 S. Kolboe, Stud. Surf. Sci. Catal., 1988, 36, 189-193.

13 B. Arstad and S. Kolboe, J. Am. Chem. Soc., 2001, 123, 8137-8138.

14 B. Arstad and S. Kolboe, Catal. Lett., 2001, 71, 209-212.

15 F. Bleken, M. Bjørgen, L. Palumbo, S. Bordiga, S. Svelle, K.-P. Lillerud and U. Olsbye, Top. Catal., 2009, 52, 218-228.

16 M. Bjørgen, U. Olsbye, S. Svelle and S. Kolboe, Catal. Lett., 2004, 93, 37-40

17 S. Svelle, U. Olsbye, F. Joensen and M. Bjorgen, J. Phys. Chem. C, 2007, 111, 17981-17984.

18 M. Bjørgen, F. Bonino, B. Arstad, S. Kolboe, K. P. Lillerud, A. Zecchina and S. Bordiga, ChemPhysChem, 2005, 6, 232-235.
19 M. Bjørgen, S. Svelle, F. Joensen, J. Nerlov, S. Kolboe, F. Bonino, L. Palumbo, S. Bordiga and U. Olsbye, J. Catal, 2007, 249, 195-207.

20 M. Bjørgen, F. Joensen, K.-P. Lillerud, U. Olsbye and S. Svelle, Catal. Today, 2009, 142, 90-97.

21 S. Teketel, S. Svelle, K.-P. Lillerud and U. Olsbye, ChemCatChem, 2009, 1, 78-81.

22 S. Teketel, U. Olsbye, K.-P. Lillerud, P. Beato and S. Svelle, Microporous Mesoporous Mater., 2010, 136, 33-41.

23 I. M. Dahl, H. Mostad, D. Akporiaye and R. Wendelbo, Microporous Mesoporous Mater., 1999, 29, 185-190.

24 F. Gramm, C. Baerlocher, L. B. McCusker, S. J. Warrender, P. A. Wright, B. Han, S. B. Hong, Z. Liu, T. Ohsuna and O. Terasaki, Nature, 2006, 444, 79-81.

25 C. Baerlocher, F. Gramm, L. Massueger, L. B. McCusker, Z. He, S. Hovmoeller and X. Zou, Science, 2007, 315, 1113-1116.

26 E. G. Derouane, P. Dejaifve, Z. Gabelica and J. C. Vedrine, Faraday Discuss., 1981, 72, 331-344.

27 J. Cejka, H. van Bekkum, A. Corma and F. Schuth, Introduction to Zeolite Science and Practice, 3rd Revised Edition. [In: Stud. Surf. Sci. Catal., 2007, 168], Elsevier B.V., 2007.

28 Z.-M. Cui, Q. Liu, W.-G. Song and L.-J. Wan, Angew. Chem., Int. Ed., 2006, 45, 6512-6515.

29 O. A. Anunziata, O. A. Orio, M. C. Aguirre and L. B. Pierella, React. Kinet. Catal. Lett., 1988, 37, 205-210.

30 O. A. Anunziata, L. B. Pierella and R. G. Marino, React. Kinet. Catal. Lett., 1995, 54, 229-237.

31 O. A. Anunziata and L. B. Pierella, React. Kinet. Catal. Lett., 1995, 55, 365-372.

32 O. Bortnovsky, P. Sazama and B. Wichterlova, Appl. Catal., A, 2005, 287, 203-213.

33 Z. Musilova-Pavlackova, M. Kubu, A. W. Burton, S. I. Zones, M. Bejblova and J. Cejka, Catal. Lett., 2009, 131, 393-400.

34 I. D. Harrison, H. F. Leach and D. A. Whan, Zeolites, 1987, 7, 21-27.

35 P. Li, W. P. Zhang, X. W. Han and X. H. Bao, Catal. Lett., 2010, 134, 124-130.

36 C. Baerlocher and L. B. McCusker, Stud. Surf. Sci. Catal., 2007, 170A, 657-665.

37 S. B. Hong, I. S. Nam, H. K. Min, C. H. Shin, S. J. Warrender, P. A. Wright, P. A. Cox, F. Gramm, C. Baerlocher, L. B. McCusker, Z. Liu, T. Ohsuna and O. Terasaki, Stud. Surf. Sci. Catal., 2007, 170A, 151-159.

38 A. Corma, A. Chica, J. M. Guil, F. J. Llopis, G. Mabilon, J. A. Perdigon-Melon and S. Valencia, J. Catal., 2000, 189, 382-394.

39 S. B. Hong, H.-K. Min, C.-H. Shin, P. A. Cox, S. J. Warrender and P. A. Wright, J. Am. Chem. Soc., 2007, 129, 10870-10885.

40 S. I. Zones, C. Y. Chen, A. Corma, M. T. Cheng, C. L. Kibby, I. Y. Chan and A. W. Burton, J. Catal., 2007, 250, 41-54.

41 S. H. Lee, D. K. Lee, C. H. Shin, Y. K. Park, P. A. Wright, W. M. Lee and S. B. Hong, J. Catal., 2003, 215, 151-170.

42 J. Abbot and B. W. Wojciechowski, Can. J. Chem. Eng., 1985, 63, $451-461$.

43 D. Chen, H. P. Rebo, K. Moljord and A. Holmen, Ind. Eng. Chem. Res., 1997, 36, 3473-3479.

44 T. V. W. Janssens, J. Catal., 2009, 264, 130-137.

45 S. B. Hong, H. K. Min, C. H. Shin, P. A. Cox, S. J. Warrender and P. A. Wright, J. Am. Chem. Soc., 2007, 129, 10870-10885.

46 S. H. Lee, D. K. Lee, C. H. Shin, Y. K. Park, P. A. Wright, W. M. Lee and S. B. Hong, J. Catal., 2003, 215, 151-170.

47 P. Magnoux, P. Roger, C. Canaff, V. Fouche, N. S. Gnep and M. Guisnet, Stud. Surf. Sci. Catal., 1987, 34, 317-330.

48 S.-H. Lee, D.-K. Lee, C.-H. Shin, Y.-K. Park, P. A. Wright, W. M. Lee and S. B. Hong, J. Catal., 2003, 215, 151-170.

49 E. P. L. Hunter and S. G. Lias, J. Phys. Chem. Ref. Data, 1998, 27, 413-656.

50 S. Svelle, P. O. Rønning, U. Olsbye and S. Kolboe, J. Catal., 2005, 234, 385-400.

51 S. Morin, N. S. Gnep and M. Guisnet, Appl. Catal., A, 1998, 168, 63-68.

52 B. P. C. Hereijgers, F. Bleken, M. H. Nilsen, S. Svelle, K. P. Lillerud, M. Bjorgen, B. M. Weckhuysen and U. Olsbye, J. Catal., 2009, 264, 77-87.

53 M. Bjørgen, U. Olsbye, D. Petersen and S. Kolboe, J. Catal., 2004, 221, $1-10$.

54 S. Svelle, F. Joensen, J. Nerlov, U. Olsbye, K. P. Lillerud, S. Kolboe and M. Bjorgen, J. Am. Chem. Soc., 2006, 128, 14770-14771. 
TNU-9 (WS201, 202, 204)
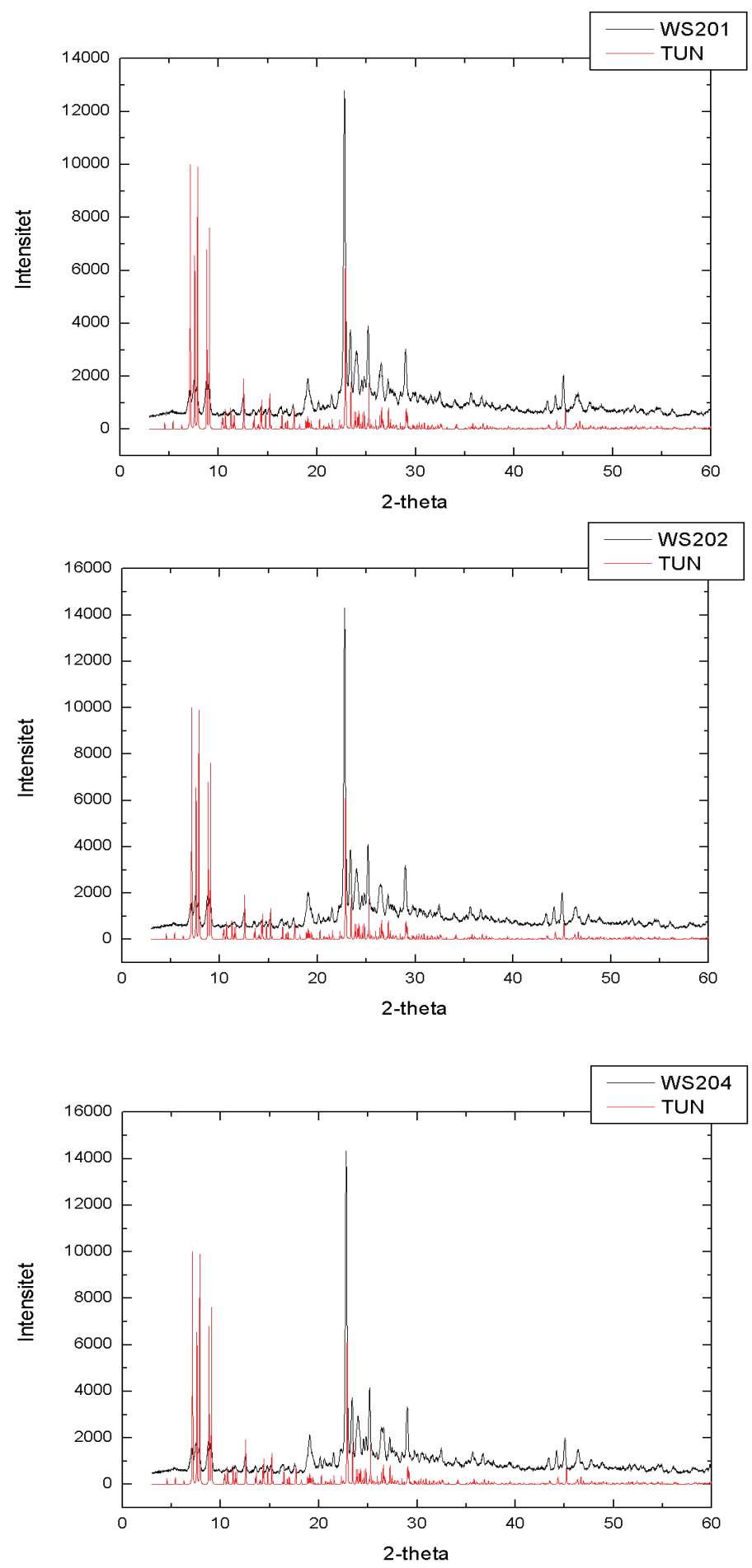


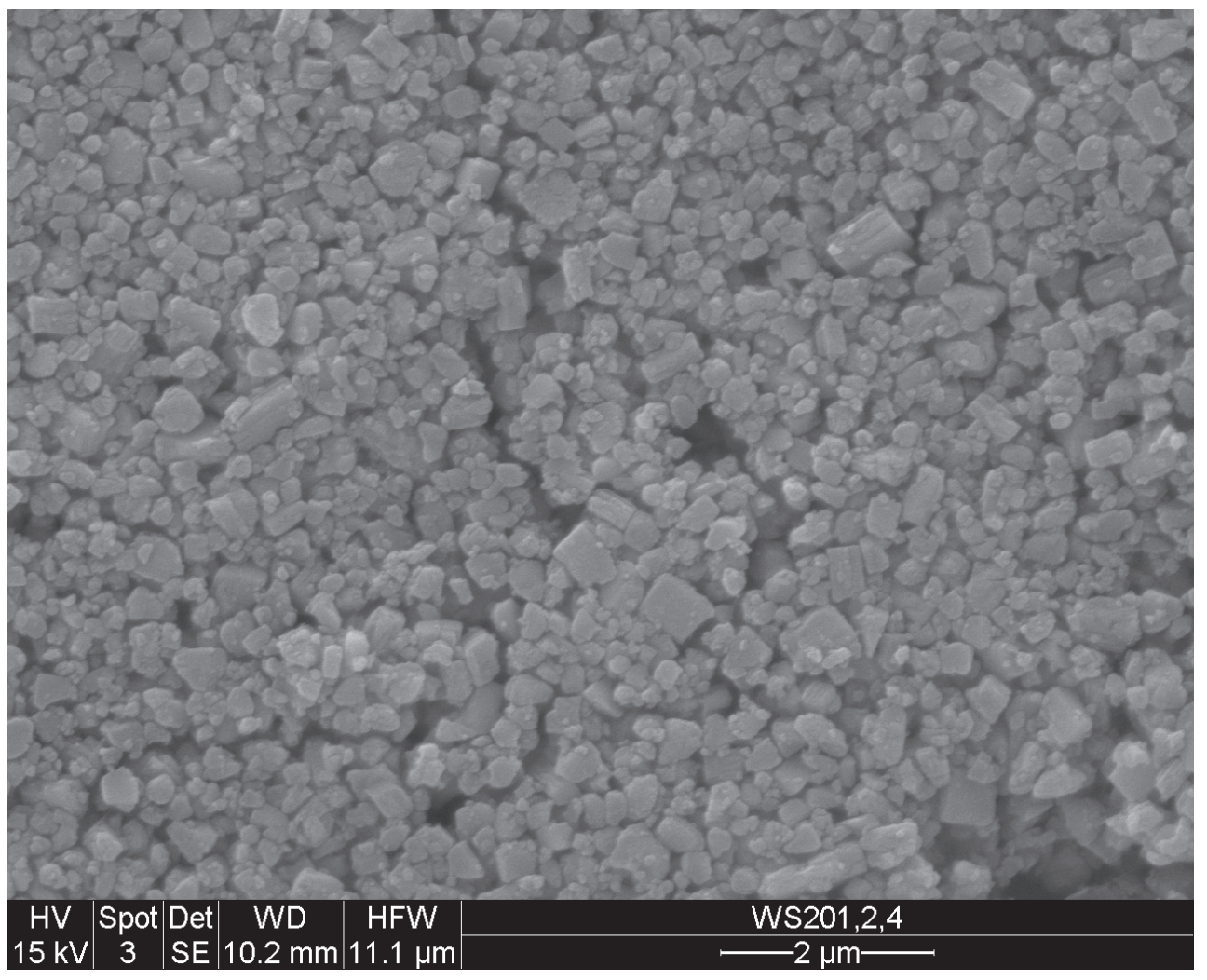




\section{IM-5 (WS142)}
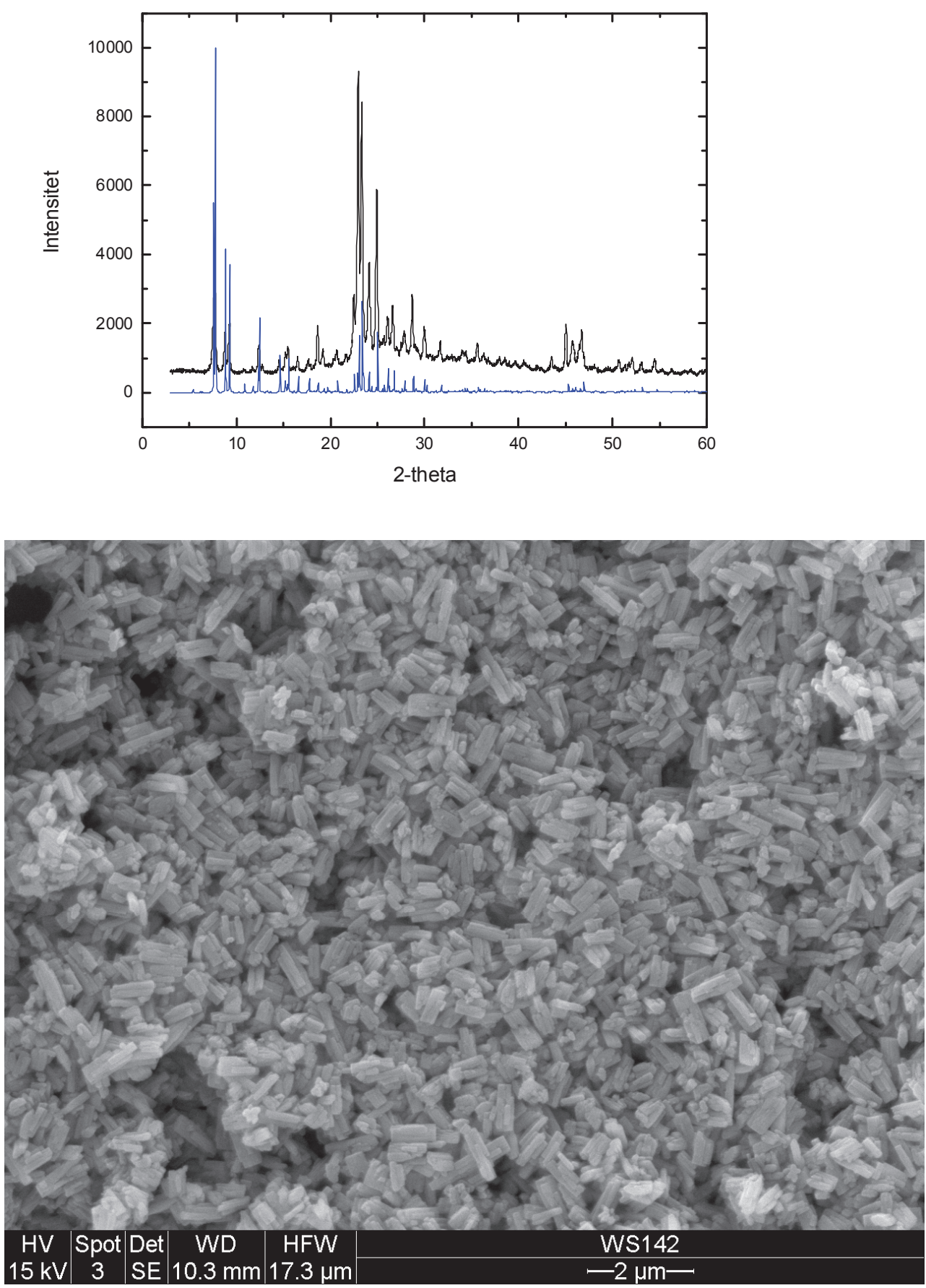
ZSM-5 (PZ-2/25-H)

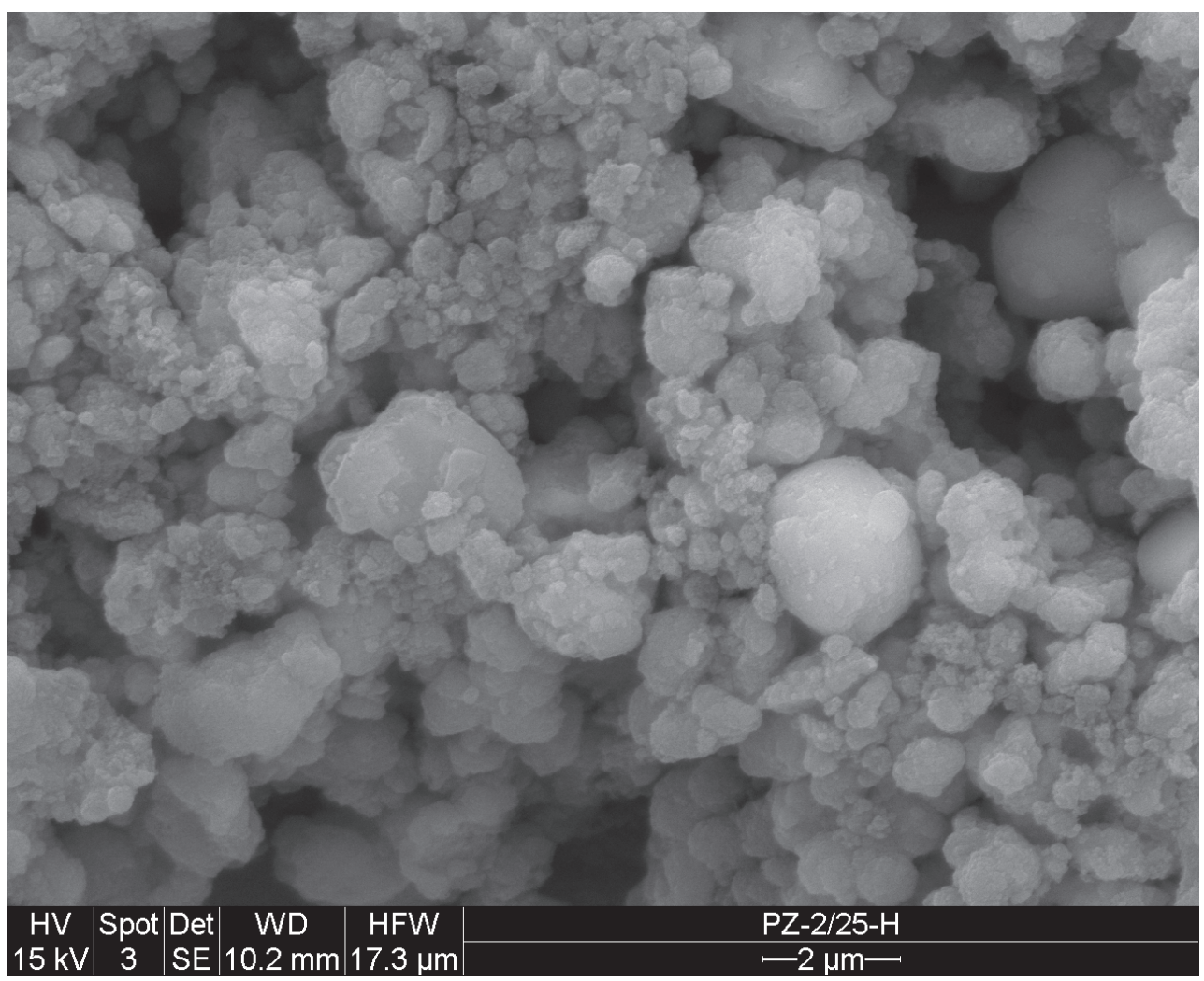




\section{ZSM-11 (MAKU10Z1)}

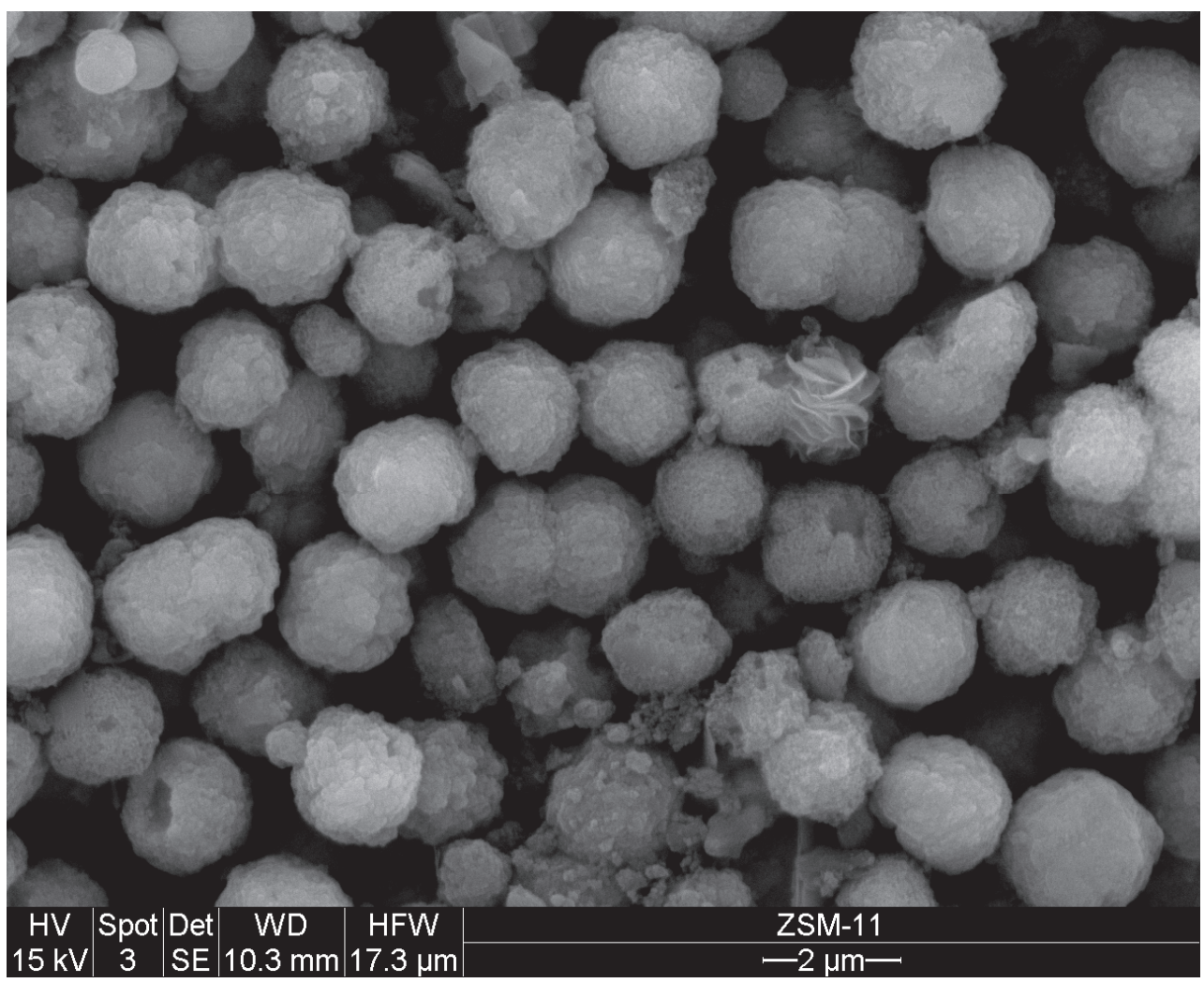




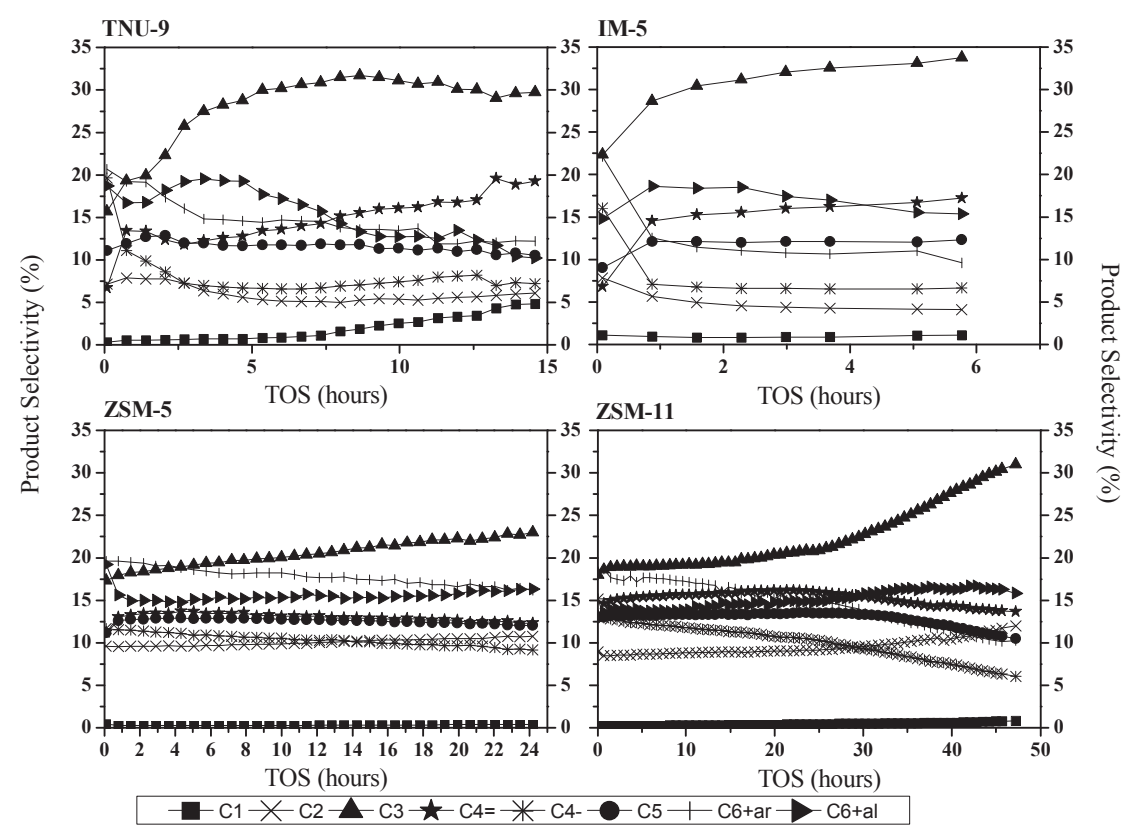

Product selectivities as function of time on stream (hours) for TNU-9 (top left), IM-5 (top right), ZSM-5 (bottom left) and ZSM-11 (bottom right). Conversion of methanol performed at $350{ }^{\circ} \mathrm{C}$ and WHSV $=9 \mathrm{~h}^{-1}$. 



\section{Preliminary manuscript I}

UV/Raman spectroscopic investigation of coke species formed over ZSM-5, TNU-9 and IM-5 in the Methanol to Hydrocarbon reactions W. Skistad, F. Bonino, P.del Campo, P. Beato, U. Olsbye, S. Svelle, K. P. Lillerud and S. Bordiga. 



\section{Manuscript I}

The influence of desilication and surfactant reassembly processes on the methanol to hydrocarbon reaction over ZSM-22, ZSM-23, EU-1,TNU-9 IM-5, ZSM-5 and ITQ-13. W. Skistad, S. Teketel, P. Beato, U. Olsbye, K. P. Lillerud and S. Svelle. 
